et comme plus haut nous avons démontré la formule (1), nous en concluons que

$$
f(E)=S_{1} S_{w} S_{8} \ldots
$$

Les ensembles $S_{n}(n=1,2,3, \ldots)$ étant ouverts, la formule (35) prouve que $f(E)$ est un ensemble $G_{\jmath}$.

Notre théorème est ainsi démontré.

On voit suns peine que toute transtormation homéomorphe $f$ d'un ensemble donné $E$ transforme tout sous-ensemble de $E$ ouvert dans $E$ en un sous-ensemble de $f(E)$ ouvert dans $f(E)$ et parsuite satisfait aux conditions de notre théorème. Il résulte done tout de suite de notre théorème la proposition suivante, due a M. Mazurkiewicz $\left.{ }^{1}\right)$ :

Un ensemble homéomorphe dे un ensemble $G_{\delta}$ est un $G_{\delta}$

Notre théorème peut donc être regardé comme une généralisation du théorème de M. Mazurkiewicz.

1) S. Mazurkiowicz: Bull. Acad. Cracovie 1916, p. 490-494. Cf. anssi: M, Lavrentioff: Fund. Math. t. VI, p. 151 et W. Sierpiński: Fund. Math. t. VIII, p. 135.

\section{Sur les classes d'ensembles closes par rapport à certaines opérations élémentaires.}

Par

\author{
Alfred Tarski (Varsovie).
}

\section{Introduction.}

Les problèmes du type suivant constituent l'objet principal des recherches exposées dans cet ouvrage: étant donné un ensemble infini $A$, combien y a-t-il de classes de sous-ensembles de $A$ pourvues d'une propriété donnée $P$ ? Les propriétés $P$, envisagées ici, ne sont d'ailleurs ni arbitraires, ni trop générales: elles reviennent toutes à ce que les classes considérées de sous-ensembles soient closes par rapport à certaines opérations elémentaires. Il s'agit notamment des opérations F qui font correspondre à toute classe d'ensembles $\boldsymbol{K}$ une nouvelle classe d'ensembles $\boldsymbol{F}(\boldsymbol{K})$ et, en. premier lieu, des opérations consistant à former les sommes, produits, différences et complémentaires des ensembles appartenant à la classe $\boldsymbol{K}$ et d'en ajouter à cette classe tous les sous-ensembles. Une classe $\boldsymbol{K}$ s'appelle close par rapport à une opération $\boldsymbol{F}$, lorsque le résultat $\boldsymbol{F}(\boldsymbol{K})$ de cette opérạtion, effectuée sur $\boldsymbol{K}$, est lui-même une sous-classe de $\boldsymbol{K}^{1}$ ).

Quelques-uns des problèmes discutés dans ce travail m'ont été posés par. MM. Poprougénko et Sierpiński.

Les principaux résultats de ces recherches sont résumés après le § 7. Je n'en mentionnerai que ceci: dans tous les problèmes envisagés dans la suite je réussis d'établir une simple relation fonctionnelle entre la puissance de la famille de toutes les

1) Cf. M. Fréchot, Des familles et fonctions additives d'ensembles, Fund. Math. IV, p. 335 
classes composées de sous-ensembles d'un ensemble infini dunné, closes par rapport à telles ou autres opérations, et la puinsance de l'ensemble donné lui-même; la puissance de cet ensemblo ́tunt a, on va voir dạns la plupart des cas que celle de la famille en question est $2^{\mathrm{a}}$ ou bien $2^{2^{\mathrm{a}}}$

Les résultats de cette nature sont énoncés dans divers théorèmes des $\S \S 3-7$, sous le nom des théorèmes fondamentaux. Au préalable, j'introduis au $\S 1$ quelques notions auxiliaires de la Théorie générale des Ensembles et j'ésquisse au $\& 2$ un algorythme concernant les opérations sur les classes d'ensembles; j'examine ensuite au $\& 3$ les propriétés générales des classes closes par rapport aux opérations quelconques, j'établis aux $\$ \S 4-7$ diverses propriétés élémentaires des opérations énumérées au début et j'y introduis aussi certainess opérations de nature auxiliaire.

Dans la majeure partie de ces recherches l'axiome du choix joue un rôle essentiel; je ne tâcherai done d'en éviter l'usage que dans les démonstrations des propriétés élémentaires des opérations en question et là, où cet axiome intervient dans le raisonnement, $j \theta$ le mentionnerai explicitement. Quelques résultats ne seront établis qu'd l'aide de l'ainsi dite hypothese de Cantor sur les alephs.

Très rapprochés des problèmes de cet ourrage, mais plus simples an point de vae logique, sont les problèmes du type suivant: étant donne un ensemble infini $A$, combien $y a-i-i l$ de sous-ensembles de $A$ ayant une propriété donnée $P$ ? Il est remarquable que tous le problèmes de ce genre, connus à l'heure actuelle, pourva qu'ils soient formulés entièrement en termes de la Théorie générale des Ensembles, se laissent aisément réduire à la forme qui suit: étant donné un ensemble infini $A$, combien y a t-il de sous-ensembles de $A$, dont la puissance jouit d'une proprieté donnée $Q$ ? $\left.{ }^{1}\right)$. Or, la question ainsi posée admet une solution générale qui s'exprime par la proposition:

$A$ étant un ensemble infini de puissance a, la classe de tous les sous-ensembles de $A$, dont la puissance jouit de la propriété $Q$, est de la puissance $\sum \mathfrak{a}^{\mathfrak{x}}$, la sommation s'étendant sur tous les nombres $\mathfrak{r}$ a proprieié $Q^{2}$ ).

1) L'observation de ce phénomène n'est a présent que parement empirique; s'il est général, il serait bien intéressant de l'expliquer et de lo préciser par den raisonnements rigoureux de la Métamathématique.

2) Cette proposition est la conséquence facile d'un théoréme connu, cit́ lel p. 195 dans la démonstration du lem. $10^{\mathrm{b}}$ du $\& 1$.
Ainsi les problèmes en question rentrent totalement dans le domaine de l'Arithmétique des Nombres Cardinaux.

\section{§ 1. Notations; notions et theorèmes auxillaires.}

Je vais me servir dans ce travail d'une série des notions et symboles connus de la Théorie générale des Ensembles sans les définir explicitement; je vais anssi m'appuyer sur diverses propriétés connues de ces notions sans citer les théorèmes qui les concernent.

Je désignerai par $a, b, \ldots, x, y, \ldots$, les individus, donc les objets qui ne sont pas des ensembles (ou dont je n'admet par l'hypothèse qu'ils soient des ensembles); par $A, B, \ldots, X, Y, \ldots$ les ensembles d'individus; par $\boldsymbol{K}, \boldsymbol{L}, \ldots, \boldsymbol{X}, \boldsymbol{Y}, \ldots$ les ensemblęs de ces ensembles, que j'appelle d'habitade classes d'ensembles; par $\mathscr{H} t, \mathfrak{L}, \ldots$, $\mathfrak{Q}, \mathscr{Y}_{1} \ldots$ les onsembles de classes d'ensembles, appelés familles de classes; par $\alpha, \beta, \ldots, \zeta, \eta, \ldots$ les nombres ordinaux et par $a, b, \ldots, r, y, \ldots$ les nombres cardinaux Les signes $f, g, h, \ldots, F, \mathcal{G}, H, \ldots, \mathcal{F}, \boldsymbol{G}, \boldsymbol{H}, \ldots, \mathcal{F}, \mathcal{Q}, \mathscr{H}, \ldots, \varphi, \psi, \chi, \ldots$ et $\mathfrak{f}, \mathfrak{g}, \mathfrak{b} \ldots$.... vont désigner les fonctions (opérations univoques) qui admettent respectivement comme valeurs: des individas, des ensembles, des classes, des familles, des nombres ordinaux ot des nombres cardinaux Dans le cas des suites du type $\alpha$, c'est-à-dire des fonctions ne définies que pour les nombres ordinaux inférieures à un nombre $a$, donné $d^{\prime} a-$ vance, je vais employer les signes de la forme $f_{\mathrm{s}}$ au tien des symboles habituels da type $f(\xi)$; outre les lettres $f, g, h, \ldots$ je me servirai dans de tels cas des lettres $a, b, \ldots, x, y, \ldots$, en écrivant p. ex. $a_{\xi}, x_{\xi}$ etc. Les classes de fonctions et de suites seront désignées respectivement par $\widetilde{F}, \mathbb{S}, \ldots$ et $\Phi, \mathbb{\Phi}, \ldots$

Je vais employer dans le sens habitael les signes et notions du Calcul des Ensembles. En particulier, les signes 0 et 1 étant reservés pour désigner les nombres ordinanx ou cardinaux, je désignerai par 0 l'ensemble vide et par 1 l'ensemble universel (composé de tons les individus qui entrent dans le domaine des considérations). Au fond, ce symbole 1 présente le caractère d'un signe variable désignant l'ensemble de tous les individus envisagés dans un thèorème donné, et, comme tel, il est susceptible aux interprétations les plus variées; cependant, pour simplifier les notations, je vais l'employer comme fixe, sans mentionner par conséquent dans les énoncés des théorémes les hypothèses telles que $a_{\in} 1$ où $A \subset 1$ et, lorsqu'il s'agit des notions gènérales, relatives à l'ensemble 1, sans mettre cette relalivité an évidence dans leurs symboles (cf. déf. 5c, 13, 14 et 16). - Pour désigner l'ensemble-somme, resp. le produit (la partie commune), de tous les ensembles d'une classe $\boldsymbol{K}$ donnée, je vais employer le symbole abregé $\boldsymbol{\Sigma}(\boldsymbol{K})$, resp. $\Pi(\boldsymbol{K})$, en dehors du symbol habitnel $\sum_{X_{\theta K}} X$, resp. $\prod_{X_{\theta} K} X$. 
Les formules $a \in A$, resp. $a \bar{\epsilon} A$ vont dire que l'élément $a$ appartient, resp. n'appartient pas à l'ensemble $A$. Pour désigner l'ensemble composé d'un seal élément $a$, je vais employer le symbole $\{a\}$ ou parfois $\mathcal{J}(a)$; les symboles $\{\dot{a}, b\},\{a, b, c\}$ etc. désigneront respectivement les ensembles composés exclusivement d'éléments $a$ et $b$ ou d'éléments $a, b$ et $c$ etc. Je vais désigner par $E[$ [] l'ensemble de toutes les valeurs d'une fonction donnée $f$ correspondant aux valeurs de l'argument $x$ qui satisfont à la condition formulée entre les parentheses [ ]; la signification du symbole $E[$ [ ] est analogue.

$\stackrel{f(x, y)}{\mathrm{De}}$ plus, je vais employer sans explication plusieurs signes et notions connues dans l'Arithmétique des Nombres Ordinaux et Cardinaux. Je vais désigner, comme d'habitude, par $\overline{\bar{A}}$ la puissance de l'ensemble $A$; je pose, en particalier,

$$
\bar{\alpha}=\overline{\bar{E}[\xi<\alpha]} \text { et } \quad \kappa_{\alpha}=\overline{\omega_{\alpha}}=\overline{E\left[\xi<\omega_{\alpha}\right]} \text {. }
$$

Je vais représenter toajours les nombres cardinaux infinis sons la forme des alephs $\aleph_{\alpha}$; cela va nous permettre de donner aux théorèmes fondam mentaux de ce travail un aspect extérieur uniforme, sans en diminner en même temps de la généralité (en raison du théorème connu de M: Zermelo sur le bon ordre).

Or, je donne maintenant les définitions explicites et j'établis certaines propriétés des notions et des signes également généraux, mais moins connus ou introduits comme nouveaux.

Définition 1. a) $\sup (A)$ est le plus petit des nombres ordinaux $\eta$ remplissant la condition: $\xi<\eta$, lorsque $\xi \in A$;

b) $\lim _{\xi<\alpha} \varphi_{\xi}=\sup \left(\underset{\varphi_{\xi}}{E}[\xi<\alpha]\right)$ dans le cas oil la suite de nombres

ordinaux $\varphi$ du type $\alpha$ est une suite croissante $\left.{ }^{{ }^{1}}\right)$ et a est un nombre de $2^{m e}$ espèce différent de 0 .

Définition 2. $c f(\alpha)$ est le plus petit des nombres ordinaux $\eta$ remplissant la condition: il existe une suite $\varphi$ du type $\omega_{\eta}$ telle que $\omega_{\alpha}=\lim _{\xi<\omega_{\eta}} \varphi_{\xi}$.

II est à rappeller à propos de la définition précédente que les normbres initiaux $\omega_{\alpha}$ sont dits réguliers ou singulier's snivant que of $(\alpha)=\alpha$
ou $f f(\alpha)<\alpha$.

1) c.-à-d. lorsque $\varphi_{\xi}<\varphi_{\eta}$ pour $\xi<\eta$.
Je vais établir quelques propriétés du symbole $c f(\alpha)$ dans les trois lemmes suivants:

Lemme 1. a) Pour tout nombre ordinal $\alpha$ on a $c f(\alpha) \leqslant \alpha$;

b) si $\alpha=0$ ou bien si $\alpha$ est un nombre de $1^{r e}$ espèce, on a cf $(\alpha)=\alpha$;

c) si $\omega_{\alpha}=\lim _{\xi<\beta} \varphi_{\xi}$, on $a \omega_{c f(\alpha)} \leqslant \beta$ et $c f(a)=c f(\beta)$;

d) $c f\left(\omega_{\alpha}\right)=\stackrel{c}{c} f(\alpha)=c f(c f(\alpha))$.

Lemme 2. a) Pour tout nombre $a$ il existe une suite de nombres cardinaux $f$ du type $\omega_{c f(\alpha)}$ vérifiant les formules: $\aleph_{\alpha}=\sum_{\xi<\omega_{c f f}(\alpha)} \mathrm{f}_{\xi}$ et $0<$ $<f_{\xi}<\kappa_{\alpha}$ pour $\xi<\omega_{q f(\alpha)}$

b) si $0<\overline{\bar{A}} \leqslant \aleph_{\alpha}$, il extste une suite croissante d'ensembles $F d u$ type $\left.\omega_{c f(a)}{ }^{1}\right)$ vérifiant los formules: $A=\sum_{\xi<\omega_{c f}(\alpha)} F_{\xi}$ et $0<\overline{\overline{F_{\xi}}}<\kappa_{\alpha}$ pour $\xi<\omega_{c / \alpha)}$.

Lemme $3 . \quad$ a) Si $\bar{B}<\aleph_{c f(a)}$ et $\mathrm{f}(x)<\aleph_{\alpha}$, pour $x \in B$, on $a$ $\sum_{x \in B} f(x)<\kappa_{\alpha}$

b) si $\bar{B}<\aleph_{f(\alpha)}$ et $\overline{\overline{F(x)}}<\aleph_{\alpha}$ pour $x \epsilon B$, on a $\overline{\overline{\sum_{x \in B} F(x)}}<\aleph_{\alpha}$;

c) si $\dot{\bar{B}}<\aleph_{\text {c( }(\alpha)}$ et $B C \sum_{\bar{\xi}<\omega_{\alpha}} F_{\xi}$, il existe un nombre $\eta$ tel que $0<\eta<\omega_{a}$ et $B \subset \sum_{\xi<\eta} F_{\xi}$.

Démonstration de ces trois lemmes, basée sur la déf. 1 , s'obtient facilement par les procédés de raisonuement connus. J'en vais démontrer à titre d'exemple le lem. $3^{c}$.

L'hypothèse du lemme entraîne la décomposition suivante de l'ensemble $B$ :

$$
B=\sum_{\xi<\omega_{\alpha}}\left(B \cdot F_{\xi}-\sum_{\eta<\xi} B \cdot F_{\eta}\right) .
$$

Considérons les nombres ordinaux $\xi$, pour lesquels les sommandés

1) c.-d-d, telle que $F_{\xi} \subset F_{\eta}$ pour $\xi<\eta<\omega_{c f(\alpha)}$. 
correspondants de la décomposition (1), c. $-\dot{a}-d$, les ensernbles $B \cdot F_{\xi}-\sum_{\eta<\xi} B \cdot F_{\eta}$, ne sont pas vides. En ordonnant ces nombres selon la grandeur, on obtient une suite $\varphi$ du type $\beta$ satisfaisant aux conditions:

$$
\begin{aligned}
& \text { (2) } \varphi_{\eta}<\varphi_{\vartheta}<\omega_{a} \text {, lorsque } \eta<\vartheta<\beta \text {, } \\
& \text { et } \\
& \text { (3) } \quad B=\sum_{\vartheta<\beta}\left(A \cdot F_{\varphi_{\vartheta}}-\sum_{\eta<\vartheta} A \cdot F_{\varphi_{\eta}}^{\prime}\right), \\
& \text { où } A \cdot F_{\varphi_{\vartheta}}-\sum_{\eta<\theta} A \cdot F_{\varphi_{\eta}} \neq 0 \text { pour } \vartheta<\beta \text {. }
\end{aligned}
$$

Si l'on avait $\beta \geqslant \omega_{a f(a)}$, la formule (3) établirait la décomposition de l'ensemble $B$ en sommandes disjunts nun-vides et en quantité $\geqslant \mathrm{s}_{c f(\alpha)}$; or, l'ensemble $B$ étant par hypothèse de puissance $<\kappa_{c f(\alpha)}$, on démontre sans peine à l'aide de l'axiome du choix qu'une telle décomposition est impossible. Donc

(4)

Supposons que

$$
\text { . } \beta<\omega_{c / \alpha)}
$$

$$
\sup \left(\underset{\varphi_{\vartheta}}{E}[\vartheta<\beta]\right)=\omega_{\alpha} .
$$

On conclut facilement de (2) et (5) que $\beta$ est un nombre de $2^{\text {mo }}$ espèce (puisqu'on aurait dans le cas contraire $\sup (E[\vartheta<\beta])=$ $=\varphi_{\beta-1}<\omega_{\alpha}$ ). Si $\beta=0$, on aurait selon (3) $B=0$ et la thèse du lemme serait évidemment remplie; nous pouvons donc admettre de plus que $\beta \neq 0$. En conséquence et conformément a (2) et d la déf. $1^{b}$ on peat écrire (5) sous la forme $\lim _{\xi<\beta} \varphi_{\xi}=\omega_{\alpha}$. En vertu du lem. $1^{\circ}$ il en résulte que $\omega_{c / \alpha)} \leqslant \beta$, ce qui est en contradiction évidente avec (4). Nous sommes donc contraints de rejeter la supposition (5) et d'admettre que

$$
\left.\sup \underset{\varphi_{\vartheta}}{(E}[\vartheta<\beta]\right) \neq \omega_{\alpha} .
$$

Les formules (2) et (16) donnent aussitôt:

$$
\left.\sup \underset{\varphi_{\vartheta}}{(E}[\vartheta<\beta]\right)<\omega_{\alpha} .
$$

En posant: $\left.\sup \underset{\varphi_{\vartheta}}{E}[\vartheta<\beta]\right)+1=\eta$, on se convaint facilement que $\eta$ est le nombre cherché: on a en effet selon (7) $0<\eta<\omega_{\alpha}$ et on déduit de (3) que $B \subset \sum_{\xi<\eta} F_{\xi}$, c. q. f. d.

Definition 3. $p(\alpha)$ est le plus petit des nombres ordinaux $\eta$ verifiant la formule: $\aleph_{\alpha}<\aleph_{a}^{N} \eta$.

Lemme 4. "a) $p(\alpha) \leqslant c f(\alpha)$

b) $c f(p(\alpha))=p(\alpha)$

-) les conditions suivantes sont équivalentes:
(1) $\beta<p(\alpha)$
(2) $\aleph_{\alpha}=\aleph_{\alpha}^{*} \beta$,
(3) il existe un nombre cardinal a tel que $\mathfrak{\aleph}_{\alpha}=\mathfrak{a}^{\aleph_{\beta}}$.

Démonstration. a) En appliquant le théorème connu de J. König (généralisé par M. Zermelo), on conclut du lem. $2^{a}$ que $\left.\aleph_{\alpha}<\kappa_{\alpha}^{\star} c / \alpha\right)$. De là, conformément à la déf. $3, p(\alpha) \leqslant c f(\alpha)$, c. q. f. d.

b) Suivant le lem. $2^{a}$ (pour le nombre $p(\alpha)$ ) il existe une suite de nombres cardinaux $f$ du type $\omega_{c f(p(\alpha))}$ vérifiant les formules:

$$
\aleph_{p(\alpha)}=\sum_{\xi<\omega_{f f(p(\alpha))}} \mathrm{f}_{\xi}
$$

$$
0<\mathrm{f}_{\xi}<\kappa_{p(\alpha)} \text { pour } \xi<\omega_{c f(p(\alpha))} .
$$

$\cap_{n}$ conclut de (1) que

$$
\kappa_{\alpha}^{x_{p}(\alpha)}=\prod_{\xi<\omega_{c f(p(\alpha))}} \kappa_{\alpha}^{\prime} \dot{\xi} .
$$

Comme on a selon (2) et d'après la déf. $3 \quad \kappa_{\alpha}^{f} \xi=\aleph_{\alpha}$ pour $\xi<\omega_{c f(p(\alpha))}$, on obtient de (3):

$$
\aleph_{a}^{\psi_{p}(\alpha)}=\aleph_{a}^{\boldsymbol{\alpha}_{f} f(p(\alpha))} \text {. }
$$

Il résulte de (4), conformément à la déf. 3, que le nombre $c f(p(\alpha))$ est, d'une façon analogue au nombre $p(\alpha)$, un des nombres $\eta$ remplissant la condition: $\aleph_{\alpha}<\aleph_{\alpha}^{N_{\eta}}$; mais comme $p(\alpha)$ est le plus petit nombre de ce genre, on a ef $(p(\alpha)) \geqslant p(\alpha)$. L'inégalité inverse, 
c.-à-d. $c f(p(\alpha)) \leqslant p(\alpha)$, présente un cas particalier du lem. 1a. On obtient ainsi finalement la formule cherchée: $c f(p(\alpha))=p(\alpha)$.

c) Les conditions (1) et (2) sont équivalentes en raison de la déf. 3 ; l'équivalence de (2) et (3) résulte de la formule connue $\left(\mathfrak{a}^{\alpha_{\beta}}\right)^{\mathbb{N}_{\beta}}=\mathfrak{a}^{\mathbb{N}_{\beta}^{2}}=\mathfrak{a}^{\mathbb{N}_{\beta}}$.

Définition 4. $\quad \mathfrak{a}^{\mathfrak{b}}=\sum_{\mathfrak{x}<\mathfrak{b}} \mathfrak{a}^{\mathfrak{k}}$.

Lemme 5. a) Si $\mathfrak{b} \leqslant \mathfrak{c}^{1}$ ), on $a$ a $\mathfrak{b} \leqslant \mathfrak{a}^{\mathfrak{c}}=\mathfrak{a}^{\mathfrak{b}}+\sum_{\mathfrak{b}, \mathfrak{b}<\mathfrak{c}} \mathfrak{a}^{\mathfrak{y}}$;

b) si $\mathfrak{a} \leqslant \mathfrak{b}$, on $a \mathfrak{a} \mathfrak{c} \leqslant \mathfrak{b} \mathfrak{c}$;

c) $s i b \geqslant 2$, on $a$ a $\stackrel{b}{2} \geqslant \mathfrak{a}$

d) si $\mathfrak{a} \geqslant 2$, on $a \mathfrak{a} \mathfrak{b} \geqslant \mathfrak{b}$.

Démonstration de a), b) et o) est éridente.

d) Pour omettre le cas évident de $\mathfrak{b} \leqslant \aleph_{0}$, posons: $\mathfrak{b}=\aleph_{\beta}$ où $\beta \neq 0$. D'après la déf. $4 \mathfrak{a}^{N_{\beta}} \geqslant \sum_{\xi<\beta} \mathfrak{a}^{N_{\xi}} \geqslant \sum_{\xi<\beta} 2^{N_{\xi}} \geqslant \sum_{\xi<\beta} \kappa_{\xi+1}=\kappa_{\beta}$, donc $\mathfrak{a}^{\mathfrak{b}} \geqslant \mathfrak{b}$, c. q. f. d.

Lemme 6. a) $S i \kappa_{p(a)} \geqslant \mathfrak{b} \geqslant 2$, on $a \cdot \aleph_{\alpha}^{b}=\aleph_{\alpha}$;

b) si $\mathfrak{a} \geqslant 2$, on $a \mathfrak{a}^{\mathrm{K}_{\beta+1}}=\mathfrak{a}^{\mathrm{N}_{\beta}}$;

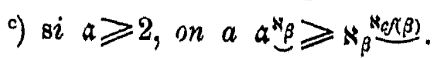

Démonstration. a) Conformément à la déf. $4 \aleph_{\alpha}^{b}=\sum_{x<b} \kappa_{\alpha}^{x}=$ $=\sum_{1 \leqslant r<b} \kappa_{\alpha}^{x} ;$ comme d'après la déf. $3 \aleph_{\alpha}^{\mathfrak{x}}=\kappa_{\alpha}$ pour $1 \leqslant r<b \leqslant \kappa_{p(\alpha)}$ on a:

$$
\aleph_{\alpha}^{\mathfrak{b}}=\aleph_{\alpha} \cdot \overline{\overline{\mathrm{E}}[1 \leqslant \mathfrak{r}<b]} .
$$

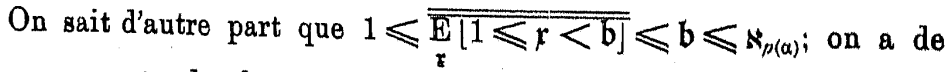
plus, en vertu des lem. $1^{\mathrm{a}}$ et $4^{\mathrm{a}}, \aleph_{p(\alpha)} \leqslant \aleph_{\alpha}$, donc

$$
1 \leqslant \overline{\mathrm{E}[1 \leqslant r<b]} \leqslant \kappa_{\alpha} \text {. }
$$

1) Nous employona ici c comme une variable (et non pour dérigner la puilsm
A l'aide du théorème connu d'Arithmétique des Nombres Cardinaux (suivant lequel $\aleph_{\alpha} \cdot \mathfrak{a}=\aleph_{\alpha}$ pour $1 \leqslant a \leqslant \aleph_{\alpha}$ ), on obtient de (1) et (2): $\aleph_{\alpha}^{\mathfrak{b}}=\aleph_{\alpha}$, c. q. f. d.

La démonstration de b) est analogue.

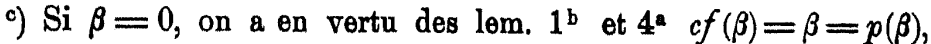
donc, conformément au lem. $6^{\mathrm{a}}, \aleph_{\beta}{ }^{\mathrm{N}_{\mathcal{C}}(\beta)}=\aleph_{\beta}^{\mathrm{N}_{p}(\beta)}=\aleph_{\beta}$, d'où en raison du lem. $5^{d} \quad a_{\beta}^{N_{\beta}} \geqslant \aleph_{\beta}=\aleph_{\beta}^{N_{c}(\beta)}$.

$\mathrm{Si} \beta$ est de $1^{\mathrm{re}}$ espèce, on obtient à l'aide du lem. $6^{\mathrm{b}}: \mathfrak{a}^{\mathrm{K}_{\beta}}=$

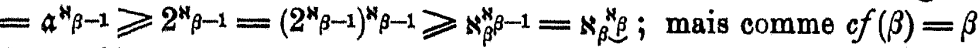
(lem. $1^{b}$ ), on a finalement $\mathfrak{a}^{k_{\beta}} \geqslant \mathcal{K}_{\beta}^{N_{c \in A}(\beta)}$.

Il reste à examiner le cas où $\beta$ est un nombre de $2^{\text {me }}$ espèce $\neq 0$. Oomme on sait ${ }^{1}$, on a dans ce cas

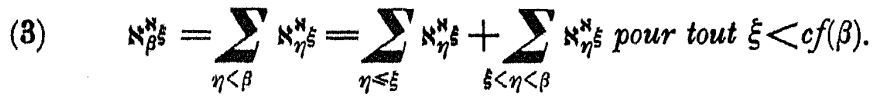

Si $\eta \leqslant \xi$, on a notoirement $\kappa_{\eta}^{\alpha_{\xi}}=2^{\alpha_{\xi}}$, d'où $\sum_{\eta \leqslant \xi} \kappa_{\eta}^{*} \leqslant 2^{\mathrm{N}_{\xi}} \cdot \overline{\xi+1} \leqslant$ $\leqslant 2^{N_{\xi}} \cdot \aleph_{\xi}=2^{\alpha_{\xi}}$; si par contre $\xi<\eta$, on obtient: $\kappa_{\eta}^{N_{\xi}} \leqslant \kappa_{\eta}^{N_{\eta}}=2^{\alpha_{\eta}}$. En tenant compte du lem. $1^{\text {a }}$, on en déduit d'après (3):

$$
\kappa_{\beta}^{*} \xi \leqslant 2^{N_{\xi}}+\sum_{\xi<\eta<\beta} 2_{\eta}^{*} \leqslant \sum_{\eta<\beta} 2^{*_{\eta}} \text { pour } \xi<c f(\beta) .
$$

D'après la déf. 4: $2^{\mathrm{N}_{\beta}} \geqslant \sum_{\eta<\beta} 2^{\mathrm{N}_{\eta}}$, d'où, en vertu de (4), $\kappa_{\beta}^{\alpha_{\xi}} \leqslant 2^{\alpha_{\beta}}$ pour $\xi<c f(\beta)$; d'autre part le lem. $5^{\mathrm{d}}$ donne: $\aleph_{\beta}^{\mathrm{x}}=\aleph_{\beta} \leqslant 2^{\mathrm{N}_{\beta}}$ pour $0<\mathrm{r}<\aleph_{0}$. Par conséquent on a généralement:

$$
\aleph_{\beta}^{*} \leqslant 2^{N_{\beta}} \text { pour } r<\kappa_{c f \beta} \text {. }
$$

A l'aide de la déf. 4 nous obtenons de (5):

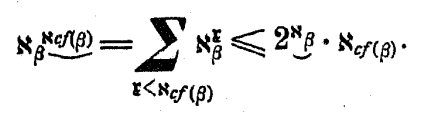

Puisque $\aleph_{q f(\beta)} \leqslant \aleph_{\beta} \leqslant 2^{\kappa_{\beta}}$ (lem. $1^{\mathrm{a}}$ et $5^{\mathrm{d}}$ ), on a $2^{\mathrm{s}_{\beta}} \cdot \aleph_{c(\beta)}=2^{\mathrm{N}_{\beta}}$, d'où en raison de (6)

$$
s_{\beta}^{{ }^{\alpha} \rho f(\beta)} \leqslant 2{ }^{N_{\beta}} .
$$

1) Cf. ma note Quelques théorèmes sur les alcphs, Fund. Math. VII, p. 7. 
Comme par hypothèse $\mathfrak{a} \geqslant 2$, on obtient du lem. $5^{\mathrm{b}}$ :

$$
a^{\mathbb{N}_{B}} \geqslant 2^{\mathbb{N}_{3}} \text {. }
$$

Il résulte aussitôt de (7) et (8) que dans le cas considéré on a également la formule cherchée:

$$
\mathfrak{a}^{\mathbb{N}_{\beta}} \geqslant \boldsymbol{s}_{\beta}{ }^{\alpha_{\sigma f}(\beta)}, \quad \text { c. q. f. d. }
$$

Le lemme 6 est ainsi entièrement démontré.

Lemme 7. a) Si $a \geqslant 2$ et $\gamma<c f(\beta)$, on $a\left(\mathfrak{A}^{\mathrm{N}}\right)^{\mathrm{N} \gamma}=\mathfrak{A}^{\mathbb{N}}$;

b) si $a \geqslant 2$ et cf $(\beta) \leqslant \gamma \leqslant \beta$, on $a\left(\mathfrak{a}^{\mathrm{N}_{\beta}}\right)^{\mathrm{N}_{\gamma}}=\mathfrak{a}^{\mathrm{N}_{\beta}}$;

c) si $a \geqslant 2$ et $\beta \leqslant \gamma$, on $a\left(\mathfrak{a}^{\mathrm{N}}\right)^{\mathrm{N} \gamma}=\mathfrak{a}^{\mathrm{N} \gamma}$.

Démonstration. a) En vertu du lem. $1^{a}$ l'inégalité $\gamma<c f(\beta)$, admise par hypothèse, entraîne $\gamma<\beta$, done $1 \leqslant \beta$. A l'aide des lem. $5^{b}$ et $6^{\text {b }}$ on en conclut que $\mathfrak{a}^{N_{\beta}}=\mathfrak{a}^{N_{1}}+\sum_{1 \leqslant \xi<\beta} \mathfrak{a}_{\xi}^{N_{\xi}}=\mathfrak{a}^{N_{0}}+\sum_{1 \leqslant j^{\prime}<\beta} \mathfrak{a}_{\xi}^{N_{\xi}}$, d'où

$$
\mathfrak{a}_{\beta}^{\mathbb{N}_{\beta}}=\sum_{\xi<\beta} \mathfrak{A}^{\mathrm{N}_{\mathfrak{\xi}}} .
$$

Étant donnés des nombres ordinaux $\xi$ et $\eta$, posons:

$$
\kappa_{(\xi, \eta)}=s_{\xi}
$$

on obtient de (1) et (2):

\section{Soit}

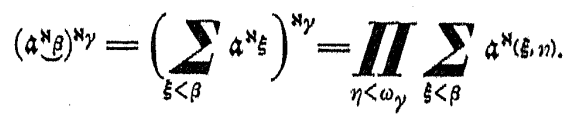

(4) Ф la classe de toutes les suites de nombres ordinaux $\varphi$ du type $\omega_{\gamma}$ remplissant la condition: $\varphi_{\eta}<\beta$ pour $\eta<\omega_{\gamma}$.

En appliquant la loi distributive générale (d'addition et de multiplication des nombres cardinaux), on déduit de (3) et (4) que

$$
\left(\mathfrak{a}^{*}\right)^{\mathbb{N}_{\gamma}}=\sum_{\varphi \in \Phi} \prod_{\eta<\omega_{\gamma}} \mathfrak{a}^{*}\left(\varphi_{\eta^{\prime}}, \eta\right)
$$

En tenant compte de (2), nous concluons de (5) que

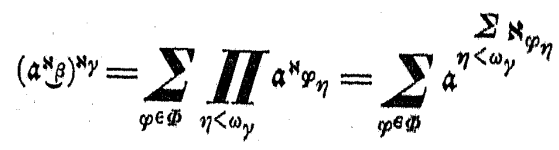

On a par hypothèse $\overline{\bar{E}\left[\eta<\omega_{\gamma}\right]}=\aleph_{\gamma}<\boldsymbol{s}_{e f(\beta)} ;$ selon (4) $\boldsymbol{s}_{\varphi_{\eta}}<\mathrm{s}_{\beta}$, lorsque $\eta<\omega_{\gamma}$ et $\varphi \in \Phi$. ${ }^{\eta}$ En posaut donc dans le lem. $3^{\mathrm{a}}: \alpha=\beta$, $B=E\left[\eta<\omega_{\gamma}\right]$ et $f(\eta)=\kappa_{\varphi_{\eta}}$, on parvient à la conclusion que $\sum_{\eta<\omega_{\gamma}} \aleph_{\varphi_{\eta}}^{\eta}<\aleph_{\beta}$, d'où en vertu de (1)

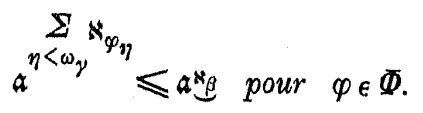

Il est à remarquer de plus que d'après la définition connue de l'exponentiation des nombres cardinaux et en raison de (4) la classe $\Phi$ est de puissance $\overline{(\beta)})^{N_{\gamma}} \leqslant \kappa_{\beta}^{N} ;$ comme en outre $\kappa_{\gamma}<\aleph_{c f(\beta)}$, on obtient conformément à la déf. 4 :

$$
\bar{\Phi} \leqslant \kappa_{\beta}^{N_{c(\alpha \beta)}} \text {. }
$$

Les formules (6)-(8) impliquent que

$$
\left(\kappa^{x_{\beta}}\right)^{N_{\gamma}} \leqslant \mathfrak{A}^{N_{\beta}} \cdot \boldsymbol{N}_{\beta}^{N_{o f f \beta}} \text {. }
$$

Il résulte du lem. $6^{\mathrm{c}}$ que $\mathfrak{a}^{\mathrm{N}_{\beta}} \geqslant \mathrm{N}_{\beta}^{\mathrm{N}_{\mathcal{G}}(\beta)}$; par conséquent (9) donne: $\left(\mathfrak{a}_{\beta}^{N_{\beta}}\right)^{\mathbb{N}_{\gamma}} \leqslant \mathfrak{A}^{\mathbb{N}_{\beta}}$. Oomme l'inégalité inverse est évidente, on a finalement:

$$
\left(\mathfrak{a}^{*}\right)^{\mathbb{N}^{*} \gamma}=\mathfrak{a}^{\mathrm{N}_{3}}, \quad \text { c. q.f. } d \text {. }
$$

b) Conformément au lem. $2^{\text {a }}$ il existe une suite de nombres cardinaux $f$ du type $\omega_{c f \beta)}$ vérifiant les formules:

$$
\aleph_{\beta}=\sum_{\xi<\omega_{f f(\beta)}} f_{\xi} \text { et } 0<f_{\xi}<\aleph_{\beta} \text { pour } \xi<\omega_{e f(\beta)} \text {. }
$$

On obtient facilement de (10):

$$
\mathfrak{a}^{\boldsymbol{\alpha}_{\beta}}=\prod_{\mathfrak{\xi}<\omega_{\mathscr{f f}(\beta)}} \mathfrak{a}^{\prime} \xi
$$

et, en vertu de la déf. 4,

$$
a^{\prime} \xi \leqslant a^{* \beta} \text { pour } \xi<\omega_{c\{(\beta)} .
$$

Comme de plus, suivant l'hypothèse du lemme, $\overline{\overline{E\left[\xi<\omega_{c f(\beta)}\right]}}=$ $=\aleph_{c /(\beta)} \leqslant \aleph_{\gamma}$, les formules (11) et (12) donnent aussitôt:

$$
\mathfrak{a}^{*_{\beta}} \leqslant\left(\mathfrak{a}^{*} \beta\right)^{* \boldsymbol{\gamma}} \text {. }
$$

D'autre part, il résulte des lem. $5^{\mathrm{a}}$ et $6^{\mathrm{b}}$ que $\mathfrak{a}_{\beta}^{\alpha_{\beta}} \leqslant \mathfrak{a}^{\alpha_{\beta+1}}=\mathfrak{a}^{\alpha_{\beta}}$, 


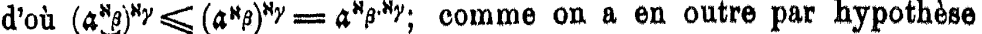
$\kappa_{\gamma} \leqslant \kappa_{\beta}$, on conclut que

$$
\left(\mathfrak{a}^{*} \beta\right)^{N \gamma} \leqslant \mathfrak{a}^{*} \beta .
$$

Les inégalités (13) et (14) entraînent immédiatement la formule cherchée: $\left(\mathfrak{a}^{\mathrm{N}_{\beta}}\right)^{\mathrm{N} \gamma}=\mathfrak{A}^{\mathrm{N} \beta}$.

c) En posant dans l'identité b) établie tout-à-l'heure $\gamma=\beta$ et en tenant compte du lem. $1^{a}$, on conclut que $\left(\mathfrak{a}^{\alpha_{\beta}}\right)^{\alpha_{\beta}}=\mathfrak{a}^{\alpha_{\beta}}$, d'où,

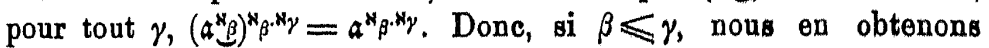
sur place:

$$
\left(\mathfrak{A}^{\boldsymbol{N}_{B}}\right)^{\boldsymbol{\alpha}_{\gamma}}=\mathfrak{a}^{\mathrm{N}_{\gamma}}, \quad \text { c. q. f. d. }
$$

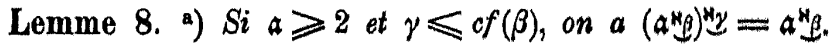

b) si $a \geqslant 2$ et of $(\beta)<\gamma \leqslant \beta+1$, on a $\left(\mathfrak{a}^{N_{3}}\right)^{\mathbf{A}_{y}}=\mathfrak{a}^{\mathrm{N}_{\beta+1}}=\mathfrak{a}^{\mathrm{N}_{\beta}}$;

c) si $\mathfrak{a} \geqslant 2$ et $\beta<\gamma$, on $a\left(\mathfrak{a}^{\alpha_{\beta}}\right)^{\alpha_{\gamma}}=\mathfrak{a}^{\boldsymbol{N}_{\gamma}}$.

Démonstration a) Conformément au lem. $7^{a}$ on a $\left(a^{N}\right)^{x}=$ $=a_{B}^{N_{B}}$ pour $s_{0} \leqslant r<\aleph_{\gamma} \leqslant \aleph_{c(\beta)} ;$ comme en outre, en vertu du lem. $5^{\mathrm{d}}, \mathfrak{a}_{3}^{\mathrm{N}_{3}}$ est un numbre transfini, cete égalité subsiste aussi dans le cas où $0<x<\kappa_{0}$. Par conséquent, on obtient à l'aide de la déf. 4:

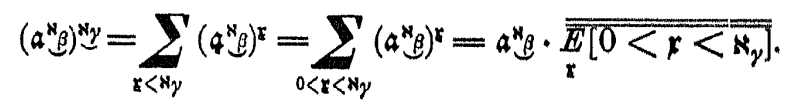

On sait d'autre part que $1 \leqslant \overline{\mathrm{E}\left[0<r<\bar{x}_{y}\right]} \leqslant \aleph_{y}$; comme on a de plus par hypothèse $\kappa_{y} \leqslant \kappa_{c \kappa(\beta)}$ et, en raison des lem. $1^{\text {a }}$ et $5^{d}$, $\aleph_{c f \beta)} \leqslant \kappa_{\beta} \leqslant \mathfrak{a}^{k_{\beta}}$, on en conclut que

$$
1 \leqslant \overline{\overline{\mathrm{E}}\left[0<\boldsymbol{r}<\overline{\left.\boldsymbol{N}_{\gamma}\right]}\right]} \leqslant \mathfrak{a}^{\mathrm{N}_{B}} .
$$

Les formules (1) et (2) donnent aussitôt: $\left(\mathfrak{a}^{*} \beta\right)^{*} \gamma=\kappa^{N}$, c. q. f. d.

$\left.{ }^{b}\right)$ En posant dans le lem. $7^{\mathrm{b}}: \gamma=c f(\beta)$ et puis $\gamma=\beta$, on obtient:

$$
\left(\mathfrak{A}^{\mathrm{N}} \beta\right)^{\mathrm{N}} \mathfrak{c f ( \beta )}=\mathfrak{a}^{\mathrm{N}} \beta=\left(\mathfrak{A}^{\mathrm{N}} \beta\right)^{\mathrm{N}} \beta
$$

En tenant compte de la formule: $c f(\beta)<\gamma \leqslant \beta+1$, admise par hypothèse, on conclut facilement des lem. $5^{\mathrm{a}}$ et $6^{\mathrm{b}}$ :

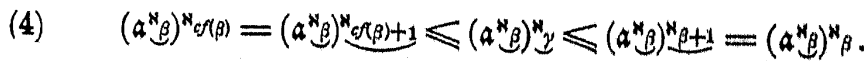

De plus le lem. $6^{b}$ donne:

$$
\mathfrak{a}^{\mathrm{N}_{\beta}}=\mathfrak{a}^{\mathrm{N}_{\beta+1}} \text {. }
$$

Les formules (3)-(5) impliquent immédiatement l'identité cherchée: $\left(\mathfrak{A}^{\alpha_{\beta}}\right)^{\boldsymbol{N}_{\gamma}}=\mathfrak{a}^{\boldsymbol{*}_{\beta+1}}=\mathfrak{A}^{\boldsymbol{\alpha}_{\beta}}$.

c) On a par hypothèse $\aleph_{\beta+1} \leqslant \Sigma_{\gamma}$; en vertu des lem. 5a et $6^{b}$ on en obtient: $\mathfrak{a}^{\alpha_{y}}=\mathfrak{a}^{\boldsymbol{H}_{\beta+1}}+\sum_{\beta+1 \leqslant \xi<\gamma} \mathfrak{a}^{\mathrm{N}_{\xi}}=\mathfrak{a}^{\boldsymbol{N}_{\beta}}+\sum_{\beta<\xi<\gamma^{\prime}} \mathfrak{a}_{\xi}^{\mathrm{N}_{\xi}}$, d'où

$$
\mathfrak{a}^{\mathrm{N}} \mathrm{y}=\sum_{\beta \leqslant \xi<\gamma} \mathfrak{a}^{\boldsymbol{N}_{\xi}} .
$$

D'une façon analogue on parvient à la formule:

$$
\left(\mathfrak{a}^{N_{\beta}}\right)^{N_{y}}=\sum_{\beta \leqslant \xi<\gamma}\left(\mathfrak{a}^{N_{\beta}}\right)^{N_{\xi}}
$$

Le lem. $7^{c}$ implique en outre que

$$
\left(\mathfrak{A}^{\mathrm{A}^{*}}\right)^{\mathrm{N}^{\mathrm{k}}}=\mathfrak{a}^{\mathrm{N}_{\xi}} \text { pour } \beta \leqslant \xi
$$

De (6)-(8) on déduit tout de suite la formule:

$$
\left(\mathfrak{a}^{*}\right)^{\mathbb{N}_{y}}=\mathfrak{a}^{\mathrm{k} y}, \text { c. q. f. } d \text {. }
$$

Dans deux derniers lemmes nous avons établis certaines simplifica-

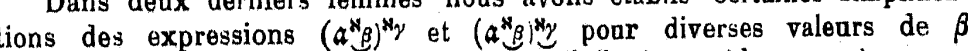
et $\gamma$. En ce qui concerne l'expression $\left(\mathfrak{a}^{\boldsymbol{\alpha}} \beta\right)^{\boldsymbol{\alpha}}$, , le problème analogue ne comporte plus de difficulté, déjà en raison da lem. $6^{\text {b }}$; nous laissons an lecteur de formuler le théorème correspondant.

Dans une partie de cet ouvrage l'hypothèse suivante $\mathrm{H}$ jouera un rôle essentiel; cette hypothèse, dite hypothèse de Cantor sur les alephs ou hypothèse du continu généralisée, n'est jusqu'à présent, comme on sait, ni démontrée, ni refutée.

Hypothèse H. Pour tout nombre ordinal $\alpha$ on a $2^{\alpha_{\alpha}}=\aleph_{\alpha+1}$.

Parmi des nombreuses conséquences de cette hypothèse, connues jusqu'à présent, il n'y a que deux qui sont d'importance pour nos considerations; or, comme nous allons voir, les deux conséquences sont équivalentes à l'hypothèse même.

Fandementa Mathematicae. T. XVI 
Lemme 9. L'hypothèse $H$ équivaut à chacune des propositions suivantes:

a) quel. que soit le nombre ordinal $\alpha$, on a $2^{\mathrm{N} \alpha}=\mathrm{s}_{\alpha}$;

b) quel que soit le nombre ordinal $\alpha$, on a $p(\alpha)=c f(\alpha)$.

Démonstration. I. Admettons que l'hypothèse $H$ est vraie.

En vertu du lem. $5^{\mathfrak{a}}$ (pour $\mathfrak{a}=2, \mathfrak{b}=\aleph_{0}$ et $\mathfrak{c}=\aleph_{\alpha}$ ) on a $2^{\star_{\alpha}}=$ $=2 \alpha^{\alpha_{a}}+\sum_{\xi<\alpha} 2^{\alpha_{\xi}}, d^{\prime} o u ̀$, conformément à la déf. 4 et à l'hypothèse $\mathrm{H}$, $2_{\alpha}^{N_{\alpha}}=\aleph_{0}+\sum_{\xi<\alpha}{\aleph_{\xi+1}}=\kappa_{\alpha}$. Donc

\section{l'hypothèse $H$ entraîne la condition a)}

II. Posons maintenant par l'hypothèse la proposition ").

En raison du lem. $7^{\mathrm{a}}$ (pour $\mathfrak{a}=2$ ) on a pour tout $\xi<c f(\alpha)$ $\left(2^{* \alpha}\right)^{\aleph_{\xi}}=2^{*_{\alpha} \alpha}$, d'où, conformément à la proposition a) $\aleph_{\alpha}^{\alpha_{\xi}^{*}}=\aleph_{\alpha}$; à l'aide du lem. $4^{\circ}$ on obtient de cette égalité: $\xi<p(\alpha)$. Nous avons ainsi montré que la formule: $\xi<c f(\alpha)$ implique toujours: $\xi<p(\alpha)$ et nous en concluons aussitôt que $c f(\alpha) \leqslant p(\alpha)$. L'inégalité inverse: $p(\alpha) \leqslant c f(\alpha)$ a été établie dans le lem. $4^{\mathrm{a}} ;$ on a donc $p(\alpha)=$ $=c f(\alpha)$ pour tout nombre $\alpha$.

Ce raisonnement prouve que

$$
\text { la condition a) entraîne la condition } \left.{ }^{b}\right) \text {. }
$$

III. Admettons enfin que la proposition b) est remplie.

Conformément au lem. $1^{\mathrm{b}}, \mathrm{c} f(\boldsymbol{\alpha}+1)=\alpha+1$, d'où suivant la condition b) $p(\alpha+1)=\alpha+1$. Il en résulte que $\alpha<p(\alpha+1)$, ce qui donne en vertu du lem. $4^{c}: \aleph_{\alpha+1}=\kappa_{\alpha+1}^{* \alpha}$. On a d'autre part

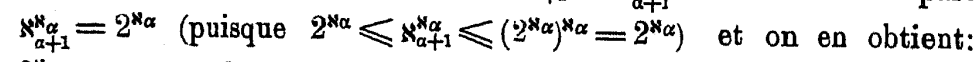
$2^{\alpha_{\alpha}}=\aleph_{a+1}$, quel que soit le nombre $\alpha$.

Nous avons ainsi prouvé que

$$
\text { la condition b) entraîne lhypothese } H \text {. }
$$

En raison de (1)-(3) le lem. 9 est entièrement dérnontré.

L'énoncé de la proposition $9^{\mathrm{b}}$ ne différe que par sa forme extérieare d'ane autre conséquence de l'hypothèse de $\mathrm{Can}$ tor:

$$
\text { si } \beta<c f(\alpha) \text {, on a } \aleph_{\alpha}^{\aleph_{\beta}}=\aleph_{\alpha} \text {, }
$$

que j'ai signalée dans mes Notes antérieures avec une démonstration un pen différente 1 ). Toutefois la forme actuelle de l'hypothèse $9^{\mathbf{b}}$ m'a été obligeamment commaniquée par M. Zermelo en 1928.

Définition 5. ") $\boldsymbol{U}(A)=\underset{\boldsymbol{X}}{\boldsymbol{E}}[X \subset A]$;

b) $V_{\alpha}(A)=\underset{X}{E}\left[X \subset A\right.$ et $\left.\frac{\bar{X}}{\bar{X}}<\aleph_{\alpha}\right]$

c) $\boldsymbol{V}(A)=\underset{X}{E}[A \subset X]$.

Lemme 10. a) $\overline{\overline{U(A)}}=2^{\overline{\bar{A}}}$;

b) si $\overline{\bar{A}} \geqslant \aleph_{\alpha}$, on a $\left.\overline{\overline{U_{\alpha}(A)}}=\overline{\overline{(A)}}\right)^{\wedge \alpha}$ et $\overline{\overline{U_{\alpha+1}(A)}}=$

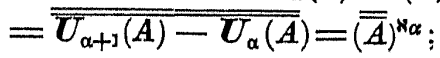

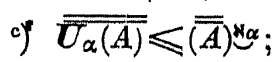

d) si $\overline{\bar{A}}=\aleph_{\alpha}$ et $\beta \leqslant p(\alpha)$, on $a \overline{\overline{U_{\beta}(A)}}=\aleph_{\alpha}$.

Démonstration. a) constitue l'énoncé du théorème connu de Oantor.

b) est basé sur un théorème connu, d'après lequel

(1) A étant un ensemble infini, si $\overline{\bar{A}} \geqslant \mathrm{~b}$, on a $\overline{\overline{E[X \subset A \text { et } \overline{\bar{X}}=\mathrm{b}]}}=$ $=(\overline{\bar{A}})^{\mathbf{b}}$ ).

Voici l'esquisse de la démonstration du théorème mentionné. Posons:

$$
\overline{\bar{E}[X \subset A \text { et } \overline{\bar{X}}=\mathrm{b}]}=\mathrm{c} \text {. }
$$

La définition de l'exponentiation des nombres cardinaux implique sans difficulté que tout ensemble de puissance $(\overline{\bar{A}})^{\mathfrak{b}}$ se laisse décomposer en $\mathrm{C}$ ensembles disjoints; à l'aide de l'axiome da choix on en obtient: $c \leqslant(\overline{\bar{A}})^{b}$.

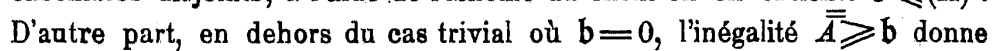
notoirement: $\overline{\bar{A}}=\overline{\bar{A}} \cdot \mathrm{b}$; en appliquant la définition de la multiplication des nombres cardinaux, on conclut. facilement que tout ensemble de puissance $\overline{\bar{A}} \cdot \mathfrak{b}$, donc, en particulier, l'ensemble $A$, admet tout au moins ${\overline{(\bar{A})^{b}}}^{b}$ sous-ensembles de puissance $b$, d'où $c \geqslant(\overline{\bar{A}} \cdot \mathfrak{b})^{\mathfrak{b}}=\left(\overline{\bar{d})^{\mathfrak{b}}}\right.$. Si l'on rap-

1) Cf. ma Note citée des Fund. Math. VII, p. 9-10, ainsi que mon article: Sur la décomposition des ensembles en sous-ensembles presque disjoints, Fund. Math. XII, p. 202.

2) Cf. W. Siorpińki, Zarys teorji mnogości (Eléments de la Théorie des Ensembles, en polonais), $1^{\text {re }}$ partie, $3^{\text {mo }}$ ed., Warszawa 1928, p. 251-253. 
proche les denx inégalités établies ci-dessus, on obtient aussitot:

$$
\overline{\bar{E}[X \subset A \text { et } \overline{\bar{X}}=\mathfrak{b}]}=\mathrm{c}=\left(\overline{\bar{A})^{\mathfrak{b}}}, \quad\right. \text { c. q. f. d. }
$$

Or, il résulte de la déf. $5^{\mathrm{b}}$ que $\boldsymbol{U}_{\alpha}(A)=\underset{X}{E}\left[X \subset A\right.$ et $\left.\overline{\bar{X}}<\kappa_{\alpha}\right]=$ $=\sum_{x<N_{a}}(\underset{x}{E}[X \subset A$ et $\overline{\bar{X}}=\mathbf{r}]) ;$ les sommandes de cette dernière somme étant disjoints, on en conclut que

$$
\overline{\overline{U_{\alpha}(A)}}=\sum_{\mathbf{r}<\star_{\alpha}} \overline{\frac{E[X \subset A \text { et } \overline{\bar{X}}=\mathbf{r}]}{E[}}
$$

Comme par hypothèse: $\overline{\bar{A}} \geqslant \aleph_{\alpha}$, les conditions (1) et (2) donnent: $\overline{\overline{V_{\alpha}(A)}}=\sum_{\mathfrak{x}<\kappa_{\alpha}}(\overline{\bar{A}})^{\mathfrak{x}}$, d'où conformément à la déf. 4 :

$$
\overline{\overline{U_{\alpha}(A)}}=(\overline{\bar{A}})^{\mathrm{Na}} \text {. }
$$

D'une façon analogue on obtient l'égalité: $\left.\overline{\overline{U_{\alpha+1}(\bar{A})}}=\overline{\overline{(A}}\right)^{\alpha_{\alpha+1}}$ et on en déduit par l'application du lem, $6^{\mathrm{b}}$ :

$$
\overline{\overline{U_{\alpha+1}(\bar{A})}}=(\overline{\bar{A}})^{\mathrm{s} \alpha} \text {. }
$$

On conclut enfin sans peine de la déf. $5^{\mathrm{b}}$ que $\boldsymbol{U}_{\alpha+1}(A)-U_{\alpha}(A)=$ $=E\left[X \subset A\right.$ et $\left.\overline{\bar{X}}=\aleph_{a}\right]$, d'où en raison de $(1)$ :

$$
\overline{\overline{U_{\alpha+1}(A)-\bar{U}_{\alpha}(A)}}=(\overline{\bar{A}})^{{ }^{\alpha} \alpha} \text {. }
$$

En vertu de $(3)-(5)$ tontes les formules $\left.{ }^{b}\right)$ se trouvent établies. c) Dans le cas où $A \geqslant \aleph_{\alpha}$ la formule: $\overline{\overline{U_{\alpha}(A)}} \leqslant(\overline{\bar{A}})^{N_{\alpha}}$ résulte immédiatement de ${ }^{\mathrm{b}}$ ). Si par contre $\overline{\bar{A}}<\aleph_{\alpha}$, on a suivant la déf. $5^{\mathrm{a}, \mathrm{b}}$ et le lem. 10a (en omettant le cas évident où $\overline{\bar{A}}<2$ ): $\overline{\overline{U_{\alpha}(\bar{A})}} \leqslant$ $\leqslant \overline{\bar{U}(\bar{A})}=2^{\overline{\bar{A}}} \leqslant\left(\overline{\bar{A})^{\bar{T}}} \leqslant \sum_{\boldsymbol{\varepsilon}<\aleph_{\alpha}}\left(\overline{\bar{A})^{x}}\right.\right.$, d'ọ̀ conformément à la déf. 4 $\overline{\overline{U_{\alpha}(\bar{A})}} \leqslant(\overline{\bar{A}})^{\mathrm{\alpha}}$, c. q. f. $\mathrm{d}$.

d) En vertu des lem. $1^{\text {a }}$ et $4^{\mathrm{a}}$ l'inégalité: $\beta \leqslant p(\alpha)$ entraîne: $\aleph_{\beta} \leqslant \aleph_{\alpha}=\overline{\bar{A}}$; en posant donc dans $\left.{ }^{b}\right): \alpha=\beta$, on conclut que $\overline{\bar{U}_{\beta}(A)}=\overline{(\bar{A})^{\aleph_{\beta}}}=\aleph_{\alpha}^{\alpha_{\beta}}$. Par l'application du lem. $6^{\mathrm{a}}$ on en obtient la formule cherchée:

$$
\overline{\overline{\boldsymbol{U}_{B}(\bar{A})}}=\aleph_{\alpha}
$$

Déflnition 6. $\bar{f}(A)=\underset{f(x)}{E}[x \in A]$ (dans lhypothèse que la fonction $f$ est définie pour tous les éléments de l'ensemble $A$ ).

L'ensemble $\bar{f}(A)$ est appelé ordinairement image de l'ensemble $A$ donnée par la fonction $f$. Divers théorèmes élémentaires sur les images d'ensembles ont été publiés antérieurement ${ }^{1}$ ); je vais établir ici quelques rapports moins connus entre les puissances des ensembles et celles de leurs images.

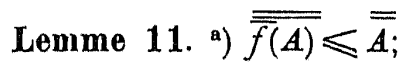

$\left.{ }^{b}\right)$ si $\overline{\overline{A \cdot E[f(x)=y]}} \leqslant \mathrm{b}$ pour tout élément $y$ de $\bar{f}(A)$, on $a \overline{A \leqslant} \overline{\overline{f(A)}} \cdot \mathrm{b}$;

c) si $\overline{\bar{A}} \geqslant \aleph_{0}$ et si $\overline{\overline{A \cdot E\lfloor f(x)=y}} \leqslant \leqslant b<\overline{\bar{A}}$ pour tout élément $y$ de $\overline{f(A)}$, on a $\overline{\overline{f(A)}}=\overline{\bar{A}}^{\text {; }}$

d) si $\overline{\bar{A}}=\aleph_{\alpha}$ et si $\overline{\overline{A \cdot E}[f(x)=y]}<\overline{\bar{A}}$ pour tout elément $y$ de $\bar{f}(A)$, on a $\overline{\overline{\overline{f(A)}}} \geqslant \aleph_{c f(\alpha)}$; si en outre of $(\alpha)=\alpha$, on a $\overline{\overline{\bar{f}(A)}}=\aleph_{\alpha}=\overline{\bar{A}}$.

Démonstration. Posons pour tout élément $y$

$$
G(y)=A \cdot \underset{x}{E}[f(x)=y]
$$

A l'aide de la déf. 6 on déduit facilement de (1) les propriétés suivantes de la fonction $G$ :

$$
\begin{gathered}
G(y) \neq 0 \text { pour } y \in \bar{f}(A) \\
\text { si } G(y) \cdot G(z) \neq 0 \text {, on a } y=z \text { et } G(y)=G(z) \text {. }
\end{gathered}
$$

En vertu de (2) et de (3) la fonction $G$ transforme d'une façon biunivoque l'ensemble $\bar{f}(A)$ dans la classe $\bar{G}(\bar{f}(A))=\underset{\sigma(y)}{E[y \epsilon} \bar{f}(A)]$; ces ensembles sont done de puissance égale:

$$
\overline{\overline{\bar{f}(A)}}=\overline{\overline{\bar{G}(\bar{f}(A))}}
$$

En raison de (1) et de la déf. 6 on obtient facilement la décomposition suivante de $A$ :

1) Cf. p. ex. A. N. Whitehoad and B. Russell, Principia Mathematica, vol, I, $2^{\text {do }}$ Ed., Cambridge $1925, * 37 . * 40$ et $* 72$. 


$$
A=\sum_{y \in \bar{f}(A)} G(y)
$$

comme selon (2) et (3) les sommandes de cette décomposition sont disjoints et non-vides, on en conclut à l'aide de l'axiome du choix que $\overline{\overline{\bar{G}(\overline{f(A))}}}=\overline{\overline{E[y \epsilon} \overline{\bar{f}(A)}]} \leqslant \overline{\bar{A}}$, d'où en vertu de (4)

$$
\overline{\overline{f(A)}} \leqslant \overline{\bar{A}} \text {. }
$$

La formule (5) donne de plus (conformément à un théorème connu de la théorie de l'égalité des puissances):

(7) $\quad$ si $\overline{\overline{G(y)}} \leqslant$ b pour $y \in \bar{f}(A)$, on $a \overline{\bar{A}} \leqslant \overline{\overline{\overline{f(A)}}} \cdot \mathfrak{b}$.

Admettons maintenant que $\overline{\bar{A}} \geqslant \aleph_{0}$ et que $\overline{\overline{G(y)}} \leqslant \mathrm{b}<\overline{\bar{A}}$ pour $y \in \bar{f}(A)$. Si l'on avait $\overline{\bar{f}(A)}<\overline{\bar{A}}$, on obtiendrait à l'aide des théorèmes connus de l'Arithmétique des Nombres Cardinaux: $\overline{\overline{f(A)}} \cdot \mathrm{b}<\overline{\bar{A}} \cdot \overline{\bar{A}}=\overline{\bar{A}}$, ce qui contredit manifestement la condition (7); par conséquent, en vertu de $(6), \overline{\overline{\bar{f}(A)}}=\overline{\bar{A}}$. On a donc:

(8) si $\overline{\bar{A}} \geqslant \aleph_{0}$ et $\overline{\overline{G(y)}} \leqslant \mathrm{b}<A$ pour $y \in \bar{f}(A)$, on a $\overline{\overline{\bar{f}(A)}}=\overline{\bar{A}}$.

Posons enfin par l'hypothèse: $\overline{\bar{A}}=\aleph_{\alpha}$ et $\overline{\overline{G(y)}}<\overline{\bar{A}}$ pour $y \epsilon \bar{f}(A)$. En remplaçant $B$ par $\overline{f(A)}$ et $F$ par $G$ dans le lem. $3^{\text {b }}$ et en tenant compte de (5), on en déduit facilement que l'inégalité $\overline{\overline{f(A)}}<\aleph_{c f(\alpha)}$ implique contradiction. On a donc $\overline{\overline{\overline{f(}(A)}} \geqslant \aleph_{c \mathcal{s}(\alpha)}$; si en outre $c f(\alpha)=\alpha$, on obtient suivant $(6): \overline{\overline{\bar{f}(A)}}=\aleph_{\alpha}=\overline{\bar{A}}$. Nous avons ainsi prouvé que

(9) si $\overline{\bar{A}}=\aleph_{\alpha}$ et $\overline{\overline{G(y)}}<\overline{\bar{A}}$ pour $y \epsilon \overline{\underline{f}}(A)$, on a $\overline{\overline{\overline{f(A)}}} \geqslant \aleph_{\text {cff(a) }}$; si de plus $c f(\alpha)=\alpha$, on a $\overline{\overline{\overline{f(A)}}}=\aleph_{\alpha}=\overline{\bar{A}}$.

En rapprochant les conditions (6)-(9) de la formule (1), on constate que le lemme en question est entièrement démontré.

Pour terminer ce $\S$, je vais étendre la notion de limite inférieure et supérieure ainsi que celle de limite proprement dite des suites infinies d'ensembles du type $\omega$ sur les suites d'ensembles du type arbitraire.
Définition 7. a) $\lim _{\xi<a} F_{\xi}=\sum_{\xi<\alpha} \prod_{\xi \leqslant \eta<\alpha} F_{\eta}$;

b) $\varlimsup_{\xi<\alpha} F_{\xi}=\prod_{\xi<\alpha} \sum_{\xi \leqslant \eta<\alpha} F_{\eta}$

c) la suite d'ensembles $F$ du type $\alpha$ est convergente et on a $\lim _{\xi<\alpha} F_{\xi}=A$, lorsque $\frac{\lim _{\xi<\alpha}}{F_{\xi}}=\varlimsup_{\xi<\alpha} F_{\xi}=A$.

Plusieurs propriétés élémentaires des notions définies tout à l'heure s'obtiennent par voie de genéralisation facile des théorèmes connus concernant les limites des suites infinies da type $\left.\omega^{1}\right)$. Je vais donc me borner à établir quelques "formules qui, autant que je sache, n'ont été jusqu'à préseant signalées. nullepart, même pour les suites infinies ordinaires.

Lemme 12. a) $\frac{\operatorname{limn}}{\xi<\alpha}\left(F_{\xi} \cdot G_{\xi}\right) \subset \frac{\lim }{\xi<\alpha} F_{\xi} \cdot \overline{\lim _{\xi<\alpha}} G_{\xi} \subset \varlimsup_{\xi<\alpha}\left(F_{\xi} \cdot G_{\xi}\right)$;

b) si les suites d'ensembles $G_{\xi}$ et $F_{\xi} \cdot G_{\xi} d u$ type a sont convergentes, la suite d'ensembles $F_{\xi} \cdot \lim _{\xi<\alpha} G_{\xi}$ est aussi convergente et on a $\lim _{\xi<\alpha}\left(\boldsymbol{F}_{\xi} \cdot \lim _{\xi<\alpha} G_{\xi}\right)=\lim _{\xi<\alpha}\left(\boldsymbol{F}_{\xi} \cdot G_{\xi}\right)$.

Démonstration. Il résulte de la déf. $7^{\mathrm{a}, \mathrm{b}}$ que $\frac{\lim }{\bar{\xi}<\alpha}\left(F_{\xi} \cdot G_{\xi}\right) \subset$ $\subset \lim _{\bar{\xi}<\alpha} F_{\xi}$ et $\left.\frac{\lim }{\bar{\xi}<\alpha}\left(F_{\xi} \cdot G_{\xi}\right) \subset \lim _{\bar{\xi}<\alpha} G_{\xi} \subset \overline{\lim }_{\xi<\alpha} G_{\xi}{ }^{8}\right)$, d'où

$$
\lim _{\bar{\xi}<\alpha}\left(F_{\xi} \cdot G_{\xi}\right) \subset \lim _{\xi<\alpha} F_{\xi} \cdot \overline{\lim }_{\xi<\alpha} G_{\xi} .
$$

D'autre part, comme on voit facilement, $\xi$ et $\xi_{1}$ étant deux nombres ordinaux tels que $\xi \leqslant \xi_{1}<\alpha$, on a $\prod_{\xi \leqslant \eta<\alpha} F_{\eta} \cdot \sum_{\xi_{1} \leqslant \eta<\alpha} G_{\eta} \subset$ $\subset \sum_{\xi \leqslant \eta<\alpha}\left(F_{\eta} \cdot G_{\eta}\right)$, d'où à plus forte raison

$$
\prod_{\xi \leqslant \eta<\alpha} F_{\eta} \cdot \varlimsup_{\eta<\alpha} G_{\eta}=\prod_{\xi \leqslant \eta<\alpha} F_{\eta} \cdot \prod_{\xi<\alpha} \sum_{\xi \leqslant \eta<\alpha} G_{\eta} \subset \sum_{\xi \leqslant \xi<\alpha}\left(F_{\eta} \cdot G_{\eta}\right) .
$$

Par conséquent pour tout nombre $\zeta<\alpha$ (non nécessairement $\geqslant \xi$ )

1) Cf. p. ex. W. Sierpinski, op, cit., p. 16-18.

2) Ce sont des généralisations des formules connues concernant des suites infinies ordinaires (cf, la note précédente). 
on a l'inclusion: $\prod_{\xi \leqslant \eta<a} F_{\eta} \cdot \overline{\lim }_{\eta<\alpha} G_{\eta} \subset \sum_{\xi \leqslant \eta<\alpha}\left(F_{\eta}^{\prime} \cdot G_{\eta}\right)$; on en obtient ensuite: $\sum_{\xi<\alpha} \prod_{\xi \leqslant \eta<\alpha} F_{\eta} \cdot \overline{\lim _{\eta<\alpha}} G_{\eta} \subset \prod_{\zeta<\alpha} \sum_{\zeta \leqslant \eta<\alpha}\left(F_{\eta} \cdot G_{\eta}\right)$, ce qui se laisse énoncer plus court à l'aide de la formule:

$$
\lim _{\bar{\xi}<\alpha} F_{\xi} \cdot \overline{\lim }_{\xi<\alpha} G_{\xi} \subset \overline{\lim }_{\xi<\alpha}\left(F_{\xi} \cdot G_{\xi}\right)
$$

Les inclusions (1) et (2) donnent aussitôt la formule cherchée a), c.-à-d.:

(3) $\quad \quad \quad \quad \frac{\lim }{\bar{\xi}<a}\left(F_{\xi} \cdot G_{\xi}\right) \subset \lim _{\bar{\xi}<a} F_{\xi} \cdot \varlimsup_{\xi<\alpha} G_{\xi} \subset \varlimsup_{\xi<\alpha}\left(F_{\xi}^{\prime} \cdot G_{\xi}\right)$.

A cause de la symétrie de la formule (3) par rapport aux deux suites $F$ et $G$ on a aussi:

$$
\lim _{\bar{\xi}<\alpha}\left(F_{\xi} \cdot G_{\xi}\right) \subset \varlimsup_{\xi<\alpha} F_{\xi} \cdot \lim _{\bar{\xi}<\alpha} G_{\xi} \subset \varlimsup_{\xi<\alpha}\left(F_{\xi} \cdot G_{\xi}\right) .
$$

Admettons à présent que les suites d'ensembles $G_{\xi}$ et $F_{\xi} \cdot G_{\xi}$ sont convergentes; conformément à la déf. 70 les formules (3) et (4) donnent alors:

$$
\lim _{\bar{\xi}<\alpha} F_{\xi} \cdot \lim _{\xi<\alpha} G_{\xi}=\varlimsup_{\xi<\alpha} F_{\xi} \cdot \lim _{\xi<\alpha} G_{\xi}=\lim _{\xi<\alpha}\left(F_{\xi} \cdot G_{\xi}\right) .
$$

Il est cependant facile de déduire de la déf. $7^{\mathrm{a}, \mathrm{b}}$ que pour tout ensemble $A$ on a $\frac{\lim }{\bar{\xi}<\alpha}\left(F_{\xi} \cdot A\right)=\lim _{\xi<a} F_{\xi} \cdot A$ et $\left.\varlimsup_{\xi<a}\left(F_{\xi} \cdot A\right)=\varlimsup_{\xi<\alpha} F_{\xi} \cdot A^{1}\right)$. Cette remarque permet d'écrire (5) sous la forme:

$$
\lim _{\bar{\xi}<\alpha}\left(F_{\xi} \cdot \lim _{\xi<\alpha} G_{\xi}\right)=\overline{\lim }_{\xi<\alpha}\left(F_{\xi} \cdot \lim _{\xi<\alpha} G_{\xi}\right)=\lim _{\xi<\alpha}\left(F_{\xi} \cdot G_{\xi}\right) .
$$

En raison de la déf. $7^{c},(6)$ exprime que la suite d'ensembles $F_{\xi} \cdot \lim _{\xi<\alpha} G_{\xi}$ est convergente et qu'on a $\lim _{\xi<\alpha}\left(F_{\xi} \cdot \lim _{\xi<\alpha} G_{\xi}\right)=\lim _{\xi<a}\left(F_{\xi} \cdot G_{\xi}\right)$; la condition b) du lemme considéré se trouve ainsi établie.

Le lem. 12b (pour $\alpha=\omega$ ) m'a été obligeamment communiqué par M. Sak s comme généralisation d'un lemme plus faible, trouvé par moi auparavant tout spécialement pour en taire usage dans les considérations présentes, alors que lem. $12^{\mathrm{a}} \mathrm{m}^{\prime} \mathrm{a}$ été encore inconna.

1) Cf. P. 199, note 1).
Comme il est aisé de vérifier, le signe de multiplication peut être remplacé parlont dans le lem. 12 par le signe d'addition.

Dans la suite j'aurai à opérer à plusieurs reprises à l'aide des notions introduites dans ce $\S$ sans en indiquer même les définitions; je vais appliquer les notions introduites dans les dét. 5 et 6 aux ensembles de tout genre (done non seulement aux ensembles d'individus, mais aussi aux classes d'ensembles, aux familles de classes etc.).

\section{§ 2. Algorythme des opérations sur les classes d'ensembles.}

Comme il a été dit dans l'introduction, nous aurons affaire dans ce travail aux certaines opérations (fonctions) $\boldsymbol{F}$ qui font correspondre à une classe quelconque d'ensembles $\boldsymbol{K}$ une autre classe $\boldsymbol{F}(\boldsymbol{K})$. L'usage de ces opérations est considérablement facilité pur un algorythme, dont je me propose d'esquisser ici les traits généraux.

Je vais introduire ell premier lieu deux relations entre opérations: celle d'identité et celle d'inclusion, en les désignant respectivement par les symboles $\stackrel{\circ}{=}$ et $\stackrel{\circ}{\complement}$.

Définition 8. a) $\boldsymbol{H} \stackrel{\circ}{=} G$, lorsqu'on a $\boldsymbol{F}(\boldsymbol{X})=\boldsymbol{G}(\boldsymbol{X})$ pour toute classe d'ensembles $\boldsymbol{X}$;

b) $\boldsymbol{F} \subset \boldsymbol{C} G$, lorsqu'on a $\boldsymbol{F}(\boldsymbol{X}) \subset \boldsymbol{G}(\boldsymbol{X})$ pour toute classe d'ensembles $\boldsymbol{X}$.

Parmi les opérations sur opérations, celle d'addition sera mise au premier plan; le résultat de cette addition sera désigné ici par $F+G ;$ resp. par $\sum_{x \in A}^{\circ} F_{x}$.

Définition 9. a) $[\boldsymbol{F}+\boldsymbol{G}](\boldsymbol{K})=\boldsymbol{F}(\boldsymbol{K})+\boldsymbol{G}(\boldsymbol{K})$;

$$
\text { b) }\left[\sum_{x \in A}^{\circ} F_{x}\right](\boldsymbol{K})=\sum_{x \in A} F_{x}(\boldsymbol{K}) \text {; }
$$

A l'usage pratique je vais supprimer parfois les parenthèses [] dans les expressions données par la déf. $9^{\mathrm{a}, \mathrm{b}}$; les autres définitions analogues seront formulées d'emblée sans ces parenthèses.

Au lieu d'établir ici une à une les lois qui concernent les notions définies tout à l'heure, je donne le lemme général suivant, dont la démonstration facile est sans doute à la portée du lecteur: 
Lemme 13. Les relations $\stackrel{\circ}{=} \complement^{\circ}$ et les opérations $\dot{+}, \dot{\sum}$ remplissent tous les postulats de l'Algèbre de la Logique ${ }^{1}$ ).

Jo définis à son tour la multiplication relative des opérations, en en désignant le résultat par $F G$, resp. par $\prod_{1 \leqslant \xi \leqslant \nu}^{0} F_{\xi}$; le produit $\prod_{1 \leqslant \xi \leqslant \nu} F_{\xi}$ dont tous les facteurs sont identiques à une opération donnée $F$

née $F$ sera appelé, comme d'habitude, $\nu^{\text {me }}$ itération (puissance) de l'opération $\boldsymbol{F}^{\prime}$ et désigné par $\boldsymbol{F}^{\boldsymbol{\nu}}$.

Definition 10. a) $\boldsymbol{F} G(\boldsymbol{K})=\boldsymbol{F}(\boldsymbol{G}(\boldsymbol{K}))$;

b) $\prod_{1 \leqslant \xi \leqslant \nu} F_{\xi}^{\prime}(K)=F_{1}(K)$ pour $\nu=1 ; \prod_{1 \leqslant \xi \leqslant \nu}^{\circ} F_{\xi}(K)=$ $\left.=\prod_{1 \leqslant \xi \leqslant \nu-1}^{0} \boldsymbol{F}_{\xi} \mid \boldsymbol{F}_{\nu}(\boldsymbol{K})\right)$ pour tout $\boldsymbol{\nu}$ tel que $1<\nu<\omega$

c) $\boldsymbol{F}^{\nu}(\boldsymbol{K})=\prod_{1 \leqslant \xi \leqslant \nu}^{\circ} \boldsymbol{F}_{\xi}(\boldsymbol{K})$ dans le cas où $1 \leqslant \nu<\omega$ et $\boldsymbol{F}_{\xi} \stackrel{\circ}{=} \boldsymbol{F}$ pour tout $\xi, 1 \leqslant \xi \leqslant \nu$.

Nous aurons bien souvent affaire au produit relatif habituel FG; c'est pourquoi je vais formuler ici explicitement quelques propriétés élémentaires de cette notion.

Par contre, la notion du produit relatif général, $\prod_{1 \leqslant \xi \leqslant v}^{\circ} F_{\xi}$, et en particulier celle de la $\boldsymbol{\nu}^{\text {ne }}$ itération d'une opération ${ }^{1 \leqslant \xi \xi}$, c.-à-d. $\boldsymbol{F}^{\nu}$, n'auront que peu d'importance dans la suite. Nous nous trourerong,

1) Systèmes de postulats de l'Algèbre de la Logique ne contenant comme seule notions primitives que celles d'identité et d'inclusion ou celles d'identité of de somme finie (somme de deux sommandes) sont à trourer dans l'article de ot de V. Hantingtion, Sets of independent postulates for the algebre of logic, Tranaact. of the Amer. Math. Soc. 5, 1904, p. 288-309. Il est aisé de modifier ces systèmes de façon qa'ils embrassent également la notion de somme gónéralisée (somme d'une classe quelconque de sommandes), notion de somme généralisée d'habitade dans l'Algèbre de la Logique. d'habitude en présence des opérations $\boldsymbol{F}$, dont toutes les itérations sont identiques et qui se laissent done caractériser par la formule: $\boldsymbol{F}^{2} \stackrel{\circ}{=} \boldsymbol{F}$; les opérations de ce genre peuvent être appelées itératives.

Lemme 14. a) $[\boldsymbol{F G}] \boldsymbol{H} \stackrel{\circ}{=}[\boldsymbol{G H}]$;

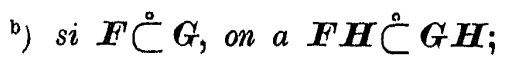

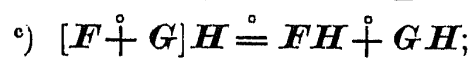

d) $\left[\sum_{\boldsymbol{F} \in \mathscr{F}}^{\circ} F\right] G \stackrel{\circ}{=} \sum_{\boldsymbol{F} \in \mathfrak{F}}^{\circ}[\boldsymbol{F G}]$;

-) si $\boldsymbol{H}^{2} \stackrel{\circ}{=} \boldsymbol{F}, \boldsymbol{G}^{2} \stackrel{\circ}{=} \boldsymbol{G}$ et $\boldsymbol{F G} \stackrel{\circ}{=} \boldsymbol{G} \boldsymbol{F}$, on a $[\boldsymbol{F G}]^{2} \stackrel{\circ}{=} \boldsymbol{F G}$.

Démonstration est évidente.

En vertu de la loi associative qui vient d’être formalée dans le lem. $13^{\mathrm{a}}$ la suppression des parenthèses dans les symboles de la forme $[\boldsymbol{F G}] \boldsymbol{H}$ ou $\boldsymbol{F}[\boldsymbol{G H}]$ ne peut canser d'équivoques.

A toute classe d'opérations $\mathfrak{F}$ viennent correspondre d'autres classes d'opérations, notamment les classes de toutes les opérations qai s'obtiennent mojeunant l'addition ou bien moyennant la multiplication relative ou enfin moyennant les deux opérations à la fois effectuées sur les éléments de la classe $\mathfrak{F}$. Je vais introduire ici des symboles spéciaux: $\mathfrak{S}(\mathfrak{F}), \mathfrak{P}(\mathfrak{F})$ et $\mathfrak{B}(\mathfrak{F})$ pour désigner les classes d'opérations ainsi obtenues.

Définition 11. a) $\mathfrak{S}(\mathfrak{F})$ est la classe de toutes les opérations $F$ qui se laissent mattre sous la forme: $\boldsymbol{F} \stackrel{\circ}{=} \sum_{G \in \mathbb{S}}^{\circ} G$, où $\mathfrak{S} \subset \mathfrak{\mho}$ et $\mathfrak{G} \neq O$;

b) $\mathfrak{B ( \Im )}$ est la classe de toutes les opérations $\boldsymbol{F}$ de la forme $F \doteq \prod_{1 \leqslant \xi \leqslant \nu}^{\circ} F_{\xi}$, oì $1 \leqslant \nu<\omega$ et $F_{\xi} \in \mathscr{\mho}$ pour $1 \leqslant \xi \leqslant \nu$;

c) $\mathfrak{B}(\mathfrak{F})=\Pi(\mathbb{E}[\mathfrak{F}+\mathfrak{S}(\mathfrak{G})+\mathfrak{B}(\mathfrak{G}) \subset \mathfrak{G}])$.

Je laisse an lectenr d’établir les propriétés générales des notions définies tout à l'heare, p. ex, les propriétés, exprimées par les formules: $\mathfrak{S}(\mathfrak{S}(\mathfrak{f}))=\mathfrak{S}(\mathfrak{F}) . \quad \mathfrak{B}(\mathfrak{P}(\mathfrak{\mho}))=\mathfrak{P}(\mathfrak{F}), \quad \mathfrak{B}(\mathfrak{B}(\mathfrak{\mho}))=\mathfrak{B}(\mathfrak{F}), \quad \mathfrak{\subseteq}(\mathfrak{F})+$ $+\mathfrak{B}(\mathfrak{F}) \subset \mathfrak{B}(\mathfrak{F})$ etc.

A propos des formules by-d) du lem. 14, il est à remarquer que l'inclusion $G \dot{C}^{\circ} \boldsymbol{H}$ n'entraîne pas toujours: $\boldsymbol{F G} \check{C}^{\circ} \boldsymbol{F H}$, de mềme 
que la loi distributive: $\mathbb{F}\left[\sum_{G \in \mathbb{S}}^{\circ} G\right] \stackrel{\circ}{=} \sum_{G \in \mathscr{S}}^{\circ}[\boldsymbol{F} G]$ (et en particulier $\boldsymbol{F}[\boldsymbol{G}+\boldsymbol{H}] \stackrel{\circ}{=} \boldsymbol{F} \boldsymbol{G}+\boldsymbol{F} \boldsymbol{H})$ n'est pas toujours vérifiée. On conuaît néanmoins nombreuses opérations $\boldsymbol{F}$ qui remplissent les conditions en question relativement aux opérations arbitruires $\boldsymbol{G}$ et $\boldsymbol{H}$ ou relativement à la classe arbitraire d'opérations (S) Les opérations de ce genre seront appelées monotones, resp. totalement additives; pour désigner les classes de toutes les opérations monotones ou additives on emploiera respectivement les signes $\mathfrak{M}$ et $\mathfrak{Z}$. Il importe de distinguer en outre les opérations des catégories intermédiaires, plus purticulières que $\mathfrak{M}$, mais plus générales que $\mathfrak{Z}$; ce sont notamment les opérations semi-additives au dégré $\beta$ ( $\beta$ étant un nombre ordinal arbitraire), dont les classes seront désignées ici par $\mathfrak{X}_{\beta}$.

Définition 12. a) $\mathfrak{M}$ est la classe de toutes les opérations $\mathbb{H}$ qui remplissent la condition: $\boldsymbol{G}$ et $\boldsymbol{H}$ étant des opérations arbitraires telles que $\boldsymbol{G} \dot{C}_{-}^{\circ} \boldsymbol{H}$, on a $\boldsymbol{F G} \stackrel{\circ}{\complement} \boldsymbol{F H}$;

b) $\mathfrak{A}$ est la classe de toutes les operations $\boldsymbol{F}$ qui remplissent la condition: $\mathfrak{G S}$ étant une classe arbitraire d'opérations, on a

$$
F\left[\sum_{G \in \mathbb{S}}^{\circ} G\right] \stackrel{\circ}{=} \sum_{G \in \mathbb{S}}^{\circ}[F G]
$$

c) $\mathfrak{X}_{\beta}$ est la classe de toutes les opérations $\boldsymbol{F}$ qui remplissent la condition: (5) etant une classe arbitraire d'opérations, on a

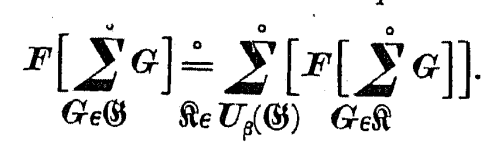

$\mathrm{La}$ déf. $12^{\mathrm{a}, \mathrm{b}}$ s'éloigne des définitions universellement admises, mais leur est néanmoins équivalente, comme le montre le suivant

Lemme 15. ") $\mathfrak{M}$ est la classe de toutes les opérations $F$ assujetties à la condition: $\boldsymbol{X}$ et $\boldsymbol{Y}$ étant des classes arbitraires d'ensembles telles que $\boldsymbol{X} \subset \boldsymbol{Y}$, on a $\boldsymbol{F}(\boldsymbol{X}) \subset \boldsymbol{F}(\boldsymbol{Y})$;

b) $\mathfrak{X}$ est la classe de toutes les opération $\mathbb{F}$ assujetties à la condition: $\mathscr{Y}$ étant une famille arbitraire de classes, on a $F(\Sigma(\mathscr{Y}))=$ $=\sum_{X_{\in} \mathscr{G}} F(X)$ c) $\mathfrak{X}_{\beta}$ est la classe de toutes les opérations $\boldsymbol{F}$ assujetties à la condition: $\boldsymbol{Y}$ etant une classe arbitraire d'ensembles, on a $\boldsymbol{F}(\boldsymbol{Y})=$

$=\sum_{\boldsymbol{X}_{\epsilon} \bar{U}_{\beta}(\boldsymbol{Y})} \boldsymbol{F}(\boldsymbol{X})$.

Démonstration. a) Que la condition du lemme est suffisante pour que $\boldsymbol{F}^{\prime} \in \mathfrak{M}$, cela résulte facilement des déf. $8^{\mathrm{b}}, 10^{\mathrm{a}}$ et $12^{\mathrm{a}}$. Pour prouver que cette condition est en mêmé temps necéssaire, on raissonnera comme suit:

Admettons que $F \in M$ et considérons deux classes arbitraires $X$ et $\boldsymbol{Y}$ telles que $\boldsymbol{X} \subset \boldsymbol{Y}$. Définissons ensuite les opérations $\boldsymbol{G}$ et $\boldsymbol{H}$, en posant p. ex.: $\boldsymbol{G}(\boldsymbol{Z})=\boldsymbol{X}$ et $\boldsymbol{H}(\boldsymbol{Z})=\boldsymbol{Y}$ pour toute classe $\boldsymbol{Z}$. Conformément à la déf. $8^{\text {b }}$ on a $G C^{\circ} \dot{H}$, d'où en raison de la déf $12^{\text {a }}$ on obtient: $\boldsymbol{F G} \mathcal{C}^{\circ} \boldsymbol{F H}$. A l'aide des déf. $8^{\mathrm{b}}$ et $10^{\mathrm{a}}$ on en conclut que $\boldsymbol{F}(\boldsymbol{G}(\boldsymbol{Y})) \subset \boldsymbol{F}(\boldsymbol{H}(\boldsymbol{Y}))$, done que $\boldsymbol{F}(\boldsymbol{X}) \subset \boldsymbol{F}(\boldsymbol{Y})$. Il est ainsi démontré que pour toute opération monotone $\boldsymbol{F}$ la formule $\boldsymbol{X} \subset \boldsymbol{Y}$ implique $\boldsymbol{F}(\boldsymbol{X}) \subset \boldsymbol{F}(\boldsymbol{Y})$, c. q. f. d.

$\left.{ }^{b}\right)$ et ${ }^{\circ}$ se démontrent d'une façon analogue.

A l'aide du lem. $15^{\mathrm{a}}$ on peut établir une série d'autres conditions, d'uillenrs bien connues, qui sunt à la fois nécessaires et suffisantes pour qu'une opération donnée $\boldsymbol{F}$ soit monotone, comme p. ex. $\boldsymbol{F}(\boldsymbol{Y})=$ $=\sum_{\boldsymbol{X}} \boldsymbol{F}(\boldsymbol{X})$ pour toute classe $\boldsymbol{Y} ; \boldsymbol{F}(\boldsymbol{X})+\boldsymbol{F}(\boldsymbol{Y}) \subset \boldsymbol{F}(\boldsymbol{X}+\boldsymbol{Y})$,

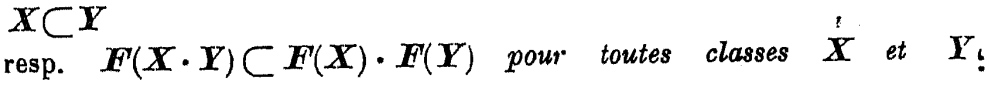
$\sum_{X_{\in} \mathscr{Y}} \boldsymbol{F}(\boldsymbol{X}) \subset \boldsymbol{F}(\boldsymbol{\Sigma}(\mathscr{Y}))$, resp. $\boldsymbol{F}(\boldsymbol{H}(\mathscr{Y})) \subset \prod_{\boldsymbol{X}_{\epsilon} \mathscr{Y}} \boldsymbol{F}(\boldsymbol{X})$ pour toute famille de classes $\mathscr{Q}$ etc; on peut en outre formuler les conditions analogues dans les termes de l'algorythme des opérations. Les opérations totalement additives peavent être caractérisées par la formule: $\boldsymbol{F}(\boldsymbol{Y})=$ $=\sum_{X_{\epsilon} \boldsymbol{Y}} \boldsymbol{F}^{\prime}(\{X\})$ pour toute classe $\boldsymbol{Y}$.

Quelques autres propriétés des classes d'opérations considérées sont tormulées dans le lemme suivant:

Lemme 16. a) $\mathfrak{A} \subset \mathfrak{X}_{\beta} \subset \mathfrak{M} ;$ si $\overline{\bar{I}}=\aleph_{\alpha}$ et $2^{\alpha_{\alpha}}<\aleph_{\beta}$, on a $\mathfrak{X}_{\beta}=\mathfrak{M}$;

b) si $\beta \leqslant \gamma$, on a $\mathfrak{X}_{\beta} \subset \mathfrak{X}_{\gamma}$ : 
c) si $\boldsymbol{F} \in \mathfrak{M}$ et $\boldsymbol{G} \in \mathfrak{M}$, on a $\boldsymbol{F}+\boldsymbol{G} \in \mathfrak{M}$ et $\boldsymbol{F G} \in \mathfrak{M} ;$ si $\mathfrak{F} \subset \mathfrak{M}$, on $a \sum_{F \in \mathfrak{F}}^{\circ} F \in \mathfrak{M}$;

d) si $\boldsymbol{F} \in \mathfrak{X}$ et $\boldsymbol{G} \in \mathcal{X}$, on a $\dot{F}+\boldsymbol{G} \in \mathfrak{Z}$ et $\boldsymbol{F G} \in \mathfrak{Z}$; si $\mathfrak{F} \subset \mathfrak{X}$, on $a \sum_{\boldsymbol{F}^{\prime} \in \mathfrak{F}}^{\circ} \boldsymbol{F}_{\boldsymbol{F}} \in \mathfrak{Z}$;

e) si $\boldsymbol{F} \in \mathfrak{I}_{\beta}$ et $\boldsymbol{G} \in \mathfrak{I}_{\beta}$, on a $\boldsymbol{F}+\boldsymbol{G}_{\epsilon} \mathfrak{I}_{\beta} ;$ si $\mathfrak{F} \subset \mathfrak{H}_{\beta}$, on $a$ $\sum_{F \in \mathfrak{F}}^{\circ} \boldsymbol{F} \in \mathfrak{X}_{\beta}$

f) si $\mathfrak{F}_{\in} \in \mathfrak{I}_{\beta}$ et $\boldsymbol{G} \in \mathfrak{I}_{\gamma}$, alors la formule: $\gamma<\beta$ implipue: $\mathbb{F G} \in \mathfrak{X}_{\beta}$, èt la formule: $\beta \leqslant \mathscr{c} f(\gamma)$ entraîne: $\boldsymbol{F G} \in \mathfrak{H}_{\gamma}$;

8) si $\boldsymbol{F} \in \mathfrak{I}_{\beta}, \quad G \in \mathfrak{X}_{\beta}$ et $f(\beta)=\beta$ ou bien si $\mathbb{F} \in \mathfrak{Z}$ et $G \in \mathfrak{X}_{\beta}$, on a $\mathbb{F G} \in \mathfrak{Z}_{\beta}$.

Démonstration. Les formules a) et b) rèsultent facilement du lem. 15 (si, en particulier, $\overline{\overline{1}}=\aleph_{\alpha}$ et $2^{\mathrm{N}_{\alpha}}<\kappa_{\beta}$, on a en raison da lem. 10a $\overline{\overline{\boldsymbol{U}_{(1)}}}=2^{\mathrm{s}_{\alpha}}<\aleph_{\beta}$, d'où $\overline{\overline{\boldsymbol{X}}}<\aleph_{\beta}$ et $\bar{U}_{\beta}(\boldsymbol{X})=\boldsymbol{U}(\boldsymbol{X})$ pour toute classe d'ensemble $X$; en le rapprochant du lem. $15^{\mathrm{a}, \mathrm{c}}$, on conclut que les formules: $\boldsymbol{F}_{\in} \mathfrak{M}$ et $\boldsymbol{F} \in \mathfrak{X}_{\beta}$ sont équivalents et parsuite que $\mathfrak{X}_{\beta}=\mathfrak{M}$ ).

Les formules $\left.{ }^{c}\right)^{-e}$ ) se déduisent de la déf. 12 à l'aide du lem. $14^{\mathrm{a}, \mathrm{c}, \mathrm{d}}$

Quant à la proposition f) on raissonnera cornme suit: Envisageons une classe arbitraire d'ensembles $\boldsymbol{K}$. Les opérations $\boldsymbol{F}^{\prime}$ et $\boldsymbol{G}$ étant par hypothèse semi-additives, elles sont à plus forte raison monotones, suivant la partie a) du théorème dont il s'agit. En vertu de o) l'opération $F G$ l'est aussi; à l'aide du lem. $15^{\mathrm{a}}$ on en conclut que $F G(X) \subset \boldsymbol{F G}(\boldsymbol{K})$ pour toute classe $\boldsymbol{X} \subset \boldsymbol{K}$. Par conséquent:
(1)
$\sum_{X_{\epsilon} D_{\beta}(K)} F G(X) \subset F G(K)$$$
{ }_{\boldsymbol{X}_{\epsilon}} \sum_{\boldsymbol{U}_{\gamma}(\boldsymbol{K})} \boldsymbol{F G}(\boldsymbol{X}) \subset \boldsymbol{F G}(\boldsymbol{K})
$$

Or, soit $Z$ un ensemble arbitraire de la classe $\boldsymbol{F} G(\boldsymbol{K})$ :

$$
Z_{\in} \boldsymbol{F G}(\boldsymbol{K})
$$

Comme $\boldsymbol{F}^{\prime} \in \mathfrak{X}_{\beta}$, on a d'après le lem. 15 $\boldsymbol{F G}(\boldsymbol{K})=\boldsymbol{\Gamma} \boldsymbol{F}(\boldsymbol{Y})$; $Y_{\epsilon} U_{\beta} G(K)$

en raison de (2) et de la déf. $5^{\text {b }}$ il existe donc une classe d'ensembles $\boldsymbol{L}$ qui vérifie les formules:

$$
\begin{gathered}
Z \in \boldsymbol{F}(\boldsymbol{L}), \\
\boldsymbol{L} \subset \boldsymbol{G}(\boldsymbol{K}) \text { et } \overline{\overline{\boldsymbol{I}}}<\aleph_{\beta} .
\end{gathered}
$$

Par une application renouvelée du lem. $15^{\mathrm{c}}$ (pour $\boldsymbol{F}=\boldsymbol{G}$ et $\beta=\gamma$ ) on obtient: $\boldsymbol{G}(\boldsymbol{K})=\sum \boldsymbol{G}(\boldsymbol{X})$. Tenant compte de (4), on $\boldsymbol{X}_{\epsilon} \widehat{\nabla}_{\gamma}(\boldsymbol{K})$

en déduit à l'aide de l'axiome du choix l'existence d'une fonction $\boldsymbol{H}$ qui fait correspondre à tout ensemble $Y$ de $\boldsymbol{L}$ une classe d'ensembles $\boldsymbol{H}(Y)$ de façon que l'on ait:

$$
\begin{gathered}
Y_{\epsilon} \boldsymbol{G} \boldsymbol{H}(Y) \text { pour } \quad Y_{\boldsymbol{\epsilon}} \boldsymbol{L} ; \\
\boldsymbol{H}(Y) \subset \boldsymbol{K} \text { et } \overline{\overline{\boldsymbol{H}(\bar{Y})}}<\aleph_{\gamma} \text { pour } Y_{\boldsymbol{\epsilon}} \boldsymbol{L} .
\end{gathered}
$$

Comme il a été déjà dit, les opérations $F^{T}$ et $G$ sont monotones; corformément au lem. $15^{\text {a }}$ et en vertu de (5) on a donc: $\boldsymbol{L} \subset \sum_{Y_{\epsilon} L} \boldsymbol{G H}(Y) \subset G\left(\sum_{Y_{\epsilon} L} \boldsymbol{H}(\boldsymbol{Y})\right)$, d'où $\boldsymbol{F}(\boldsymbol{L}) \subset \boldsymbol{F G}\left(\sum_{Y_{\epsilon} \boldsymbol{L}} \boldsymbol{H}(\boldsymbol{Y})\right) ;$

en le rapprochant de (3) et (6), on obtient:

$$
Z \in \boldsymbol{F G}\left(\sum_{Y \in L} \boldsymbol{H}(Y)\right), \text { oì } \sum_{Y \in L} \boldsymbol{H}(\boldsymbol{Y}) \subset \boldsymbol{K} .
$$

Or, soit $\gamma<\beta$, d'où $\aleph_{\gamma}<\aleph_{\beta}$. D'après (4) et (6) on a alors $\overline{\overline{\sum_{Y_{\epsilon} L} \boldsymbol{H}(Y)}} \leqslant \aleph_{\gamma} \cdot \overline{\overline{\boldsymbol{L}}}<\aleph_{\beta}^{2}=\aleph_{\beta}$. Suivant $(7)$ on en conclut que

$$
Z_{\boldsymbol{X}} \sum_{\boldsymbol{X}} \boldsymbol{F G}(\boldsymbol{X}) \text { pour } \quad \gamma<\beta
$$

Si par contre $\beta \leqslant c f(\gamma)$, on a en vertu de $(4)$ : $\overline{\bar{L}}<\aleph_{c(\gamma)}$. En posant done dans le lem. $3^{\text {b }}: B=L$ et $F \stackrel{\circ}{=}$ et en tenant 
compte de (6), on parvient à la formule: $\overline{\overline{\sum_{\epsilon \in L} \boldsymbol{H}(Y)}}<\boldsymbol{\aleph}_{\gamma}$, qui, rapprochée de (7), entraîne:

$$
\sum_{Y_{\epsilon} L}
$$

$$
Z_{\boldsymbol{X}} \sum_{\boldsymbol{U _ { \gamma }}(\boldsymbol{K})} \boldsymbol{F G}(\boldsymbol{X}) \text { pour } \beta \leqslant c f(\gamma) .
$$

Il est ainsi prouvé que (2) implique (8) et (9) pour tout $Z$. A l'aide de (1) on en déduiț les égalités:

$$
\begin{aligned}
& \text { (10) } F G(\boldsymbol{K})=\sum_{\boldsymbol{X}_{\epsilon} \boldsymbol{U}_{\beta}(\boldsymbol{K})} \boldsymbol{F G}(\boldsymbol{X}) \text { pour } \gamma<\beta ; \quad \boldsymbol{F G}(\boldsymbol{K})=\sum_{\boldsymbol{X}_{\epsilon} \boldsymbol{U}_{\gamma}(\boldsymbol{K})} \boldsymbol{F G}(\boldsymbol{X}) \\
& \text { pour }, \beta \leqslant c f(\gamma) \text {. }
\end{aligned}
$$

En conséquence du lem. $15^{\circ}$ les formules (10), qui sont valables pour toute elasse d'ensembles $\boldsymbol{K}$, donnent finalement:

$$
\boldsymbol{F G} \in \mathfrak{I}_{\beta} \text { pour } \gamma<\beta \text { et } \boldsymbol{F G} \in \mathfrak{X}_{\gamma} \text { pour } \beta \leqslant f f(\gamma) \text {, c. q. f. d. }
$$

Or, si l'on pose dans la proposition f), qui vient d'être établie: $\gamma=\beta$, resp. $\beta=0$ et $\gamma=\beta$ (et si l'on tient compte de a)), on parvient immédiatement à la proposition $\mathfrak{z}$ ).

Le lem. 16 est ainsi entièrement démontré.

En tenant compte de la déf. 11, le lem. $16^{\mathrm{c}, d, \Theta}$ peat âtre énoncé plus court à l'aide des formules: $\mathfrak{S}(\mathfrak{M})=\mathfrak{B}(\mathfrak{M})=\mathfrak{B}(\mathfrak{M})=\mathfrak{M}$,
$\mathfrak{S}(\mathfrak{A})=\mathfrak{P}(\mathfrak{I})=\mathfrak{B}(\mathfrak{I})=\mathfrak{Z}$ et $\mathfrak{S}\left(\mathfrak{I}_{\beta}\right)=\mathfrak{A}_{\beta} ;$ d'après lo lem $16^{\mathrm{g}}$ on a de plus: $\mathfrak{P}\left(\mathfrak{X}_{\beta}\right)=\mathfrak{B}\left(\mathfrak{X}_{\xi}\right)=\mathfrak{X}_{\beta}$, lorsque of $(\boldsymbol{\beta})=\boldsymbol{\beta}$.

En dehors des classes d'opérations semi-additives au dégré $\beta$ on pent considérer autres classes d'opërations également plus étroites que $\mathfrak{M}$ et plus vastes que $\mathfrak{Z}$. Comme exemple d'ane telle classe on pent citer celle des opérations additives au sens restreint, c.-à-d. des opérations $F$ qui vérifient la formule: $\boldsymbol{F}[\boldsymbol{G}+\boldsymbol{H}] \stackrel{\circ}{=} \boldsymbol{F G}+\boldsymbol{F H}$ pour toutes operations $\boldsymbol{G}$ et $\boldsymbol{H}$ (resp. $\boldsymbol{F}^{\prime}(\boldsymbol{X}+\boldsymbol{Y})=\boldsymbol{F}(\boldsymbol{X})+\boldsymbol{F}(\boldsymbol{Y})$ pour toutes classes $\boldsymbol{X}$ et $\boldsymbol{Y}$ ). En généralisant cette notion, on parvient à celle des opérations additives au dégré $\beta$, c.-à-d. caractérisables par la condition:

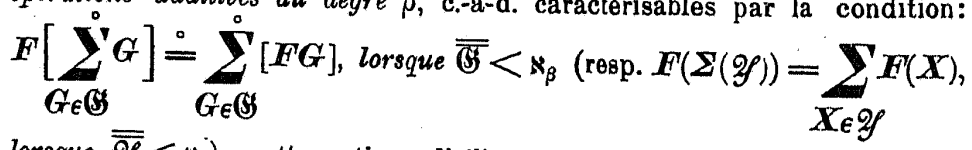
lorsque $\left.\overline{\overline{\mathscr{P}}}<\aleph_{\beta}\right)$; celte notion, d'ailleurs importante, restera cependant sans application dans les questions qui nous occupent.

Comme exemples des opérations effectuées sur les classes d'ensembles et qui sont totalement additives, donc qui présentent par cela-même toutes les autres propriétés envisagées plus haut, on peut citer toules les opérations de la forme $\bar{F}$ (cf. la déf. 6 ), où $F$ est une opération arbitraire faisant correspondre les ensembles d'individus anx ensembles d'individus. :

En rapport avec la multiplication des opérations joue un certain rôle l'opération d'identité $I$, qui en est le module. Il est à remarquer que presque toutes les opérations $F$ dont nous auvons affaire dans ces considérations vérifient l'inclusion $I \complement^{\circ} \boldsymbol{F} ;$ l'opération $I$ constitue donc à un certain point de vue leur borne inférieure. Les opérations $\boldsymbol{F}$ qui remplissent la formule: $\boldsymbol{I} \complement^{\circ} \boldsymbol{F}$ peuvent être appelées adjonctives.

\section{Définition 13. $I(K)=K$.}

Voici quelques propriétés de l'opération $\boldsymbol{I}$ et des opérations adjonctives:

Lemme 17. a) $I_{\in} \mathfrak{Z}$, donc $I_{\epsilon} \mathfrak{Z}_{\beta}$ et $I_{\epsilon} \mathfrak{M}$;

b) $\boldsymbol{I F} \stackrel{\circ}{=} \stackrel{\circ}{=} \boldsymbol{F} \boldsymbol{X}$, en particulier $\boldsymbol{I}^{2} \stackrel{\circ}{=} \boldsymbol{I}$;

c) si $\boldsymbol{I} \check{C}^{\circ} \boldsymbol{F}$, on a $\boldsymbol{G} \check{\complement}^{\circ} \boldsymbol{F G}$; si de plus $\boldsymbol{G} \in \mathrm{M}$, on a $\boldsymbol{G} \check{C}^{\circ} \boldsymbol{G F}$;

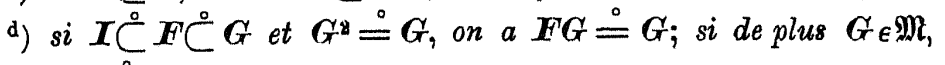
on $a \boldsymbol{G} \boldsymbol{F} \stackrel{\circ}{=} G$;

e) si $\boldsymbol{I} \complement^{\circ} \boldsymbol{F}$ et $\boldsymbol{I} \subset \mathbf{C}$, on a $\boldsymbol{I} \complement^{\circ} \boldsymbol{F G}$;

f) si $\boldsymbol{I} \subset$ ㅁ $\boldsymbol{F}$, on a $\boldsymbol{K} \subset \boldsymbol{F}(\boldsymbol{K})$ et $\overline{\overline{\boldsymbol{K}}} \leqslant \overline{\overline{\boldsymbol{F}(\boldsymbol{K})}}$.

Il est à noter que toutes les notions définies jusqu'ici dans ce § sont applicables non seulement aux opérations qui font correspondre les classes d'ensembles aux classes d'ensembles, mais aussi à toute opération sur des ensembles quelconques et ayant pour résultat des ensembles quelconques; en ce qui concerne la déf. 10 , même une telle restriction des résultats des opérations n'est pas essentielle. Un maniement systématique avec les notions introduites ici et employées dans une étendue la plus vaste rendrait possible une déscription très brève, bien que peu intuitive, de plusieurs faits acquis. Ainsi p.ex. les opérations monotones pourraient. être caractérisées par une des formules: $\overline{\boldsymbol{F}} \boldsymbol{U} \subset^{\circ} \boldsymbol{U} \boldsymbol{F}, \boldsymbol{F}=\Sigma \overline{\boldsymbol{F}} \boldsymbol{U}, \Sigma \overline{\boldsymbol{F}} \subset^{\circ} \boldsymbol{F} \boldsymbol{\Sigma}$ ou $\boldsymbol{F} \Pi \complement^{\circ} \Pi \overline{\boldsymbol{F}}$, les opérations totalement additives - par une des formules: $F^{\circ}=\Sigma \bar{F} \bar{J}$ ou $\Sigma \bar{F} \doteq F \Sigma$, et enfin les opérations semi-additives du dégré $\beta-$ - par la formule: $\boldsymbol{F}^{\circ}=\Sigma \overline{\boldsymbol{F}} \boldsymbol{U}_{\beta}$. Cependant, pour éviter de rendre la lecture de cet ouvrage plus difficile encore, je ne vais pas abuser davantage de la symbolique de ce genre; ce n'est que la notion de multiplication relative (déf. 10 $10^{\mathrm{a}}$ ) dont je ferai l'usage le plus vaste possible, ce qui me permet $p$. ex. de supprimer les parenthèses extérieures dans toutes les expressions de la forme $f(g(x))$.

Fundamenta Malhomaticae. T. XvI. 
En dehors de l'opération $\boldsymbol{I}$, une autre opération particulière est d'importance considérable pour la suite, à savoir l'opération $\boldsymbol{C}$ consistant à remplacer les ensembles $X$ d'une classe $\boldsymbol{K}$ par leurs complémentaires $1-X$.

Définition 14. $C(K)=\underset{1-X}{E}[X \in K]$.

Passons en revue quelques propriétés élémentaires de l'opération $\boldsymbol{C}$ :

Lemme 18, a) $C_{\epsilon} \mathfrak{Z}$, donc $C_{\epsilon} \mathfrak{Z}_{\beta}$ et $C_{\epsilon} \mathfrak{M}$

b) $\boldsymbol{C}^{2} \stackrel{\circ}{=} \boldsymbol{I}$;

c) $[I+C]^{2} \stackrel{\circ}{=} I+C$

d) $\boldsymbol{C}(0)=0$ et $\boldsymbol{C U}(1)=\boldsymbol{U}(1)$

e) $\overline{\overline{\boldsymbol{C}}(\overline{\boldsymbol{K}})}=\overline{\overline{\boldsymbol{K}}}$

Démostration de ${ }^{a},{ }^{b}$ ) et ${ }^{d}$ ) est évidente; ${ }^{c}$ ) peut être déduit de $\left.{ }^{a}\right)$ et $\left.{ }^{b}\right)$ à l'aide des déf. 10 et $12^{\mathrm{b}}$ et des lem. $14^{\mathrm{c}}$ et $17^{\mathrm{b}}$ La formule o) résulte du fait que la fonction $F(X)=1-X$ est biunivoque.

A toute opération $\boldsymbol{F}^{\prime}$ on peut faire correspondre une autre opération $C F C$, qui conserve beaucoup de propriétés de l'opération $\boldsymbol{F}$; cette opération $C F C$ portera le nom d'opération double de $\mathbb{F}$ et sera désignée par $F^{*}$. Je vais faire de cette notion de dualité un usage constant dans les chapîtres qui vont suivre; grâce à ses propriétés, beaucoup de raisonnements subissent une simplification considérable.

Définition 15. $\boldsymbol{F}^{* *}(\boldsymbol{K})=\operatorname{CFC}(\boldsymbol{K})$.

Certaines propriétés des opérations doubles sont rénnies dans le suivant

Lemme 19. a) $C F C \stackrel{\circ}{=} H^{*}, C H \stackrel{\circ}{=} H^{*} C, C^{*} \stackrel{\circ}{=} C$;

b) $F^{* *} \stackrel{\circ}{=} F$;

c) si $F^{\circ} \mathcal{C}^{\circ}$, on a $F^{*} \stackrel{\complement}{\complement}^{* *}$;

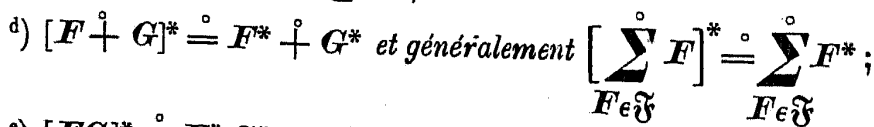

๑) $[\boldsymbol{F G}]^{*} \stackrel{\circ}{=} \boldsymbol{F}^{*} \boldsymbol{G}^{*}$

f) si $\boldsymbol{F}^{*} \stackrel{\circ}{=} \boldsymbol{F}$, on $a\left[\boldsymbol{H}^{*}\right]^{2} \stackrel{\circ}{=} \boldsymbol{F}^{*}$; в) si $F_{e} \in \mathfrak{M}$, on a $\mathbb{F}^{*} \in \mathfrak{M}$;

b) si $\mathbb{F}^{\prime} \in \mathfrak{Z}$, on a $\mathbb{F}^{*} \in \mathfrak{Z}$;

i) si $\boldsymbol{F}_{\boldsymbol{1}} \in \mathfrak{Z}_{\beta}$, on a $\boldsymbol{H}^{*} \in \mathfrak{A}_{\beta}$;

j) $\boldsymbol{I}^{*} \stackrel{\circ}{=} \boldsymbol{I}$; si $\boldsymbol{I} \check{\complement}^{\circ} \boldsymbol{F}$, on a $\boldsymbol{I} \check{\complement}^{\circ} \boldsymbol{H}^{*}$;

k) si $F^{\prime}(0)=0$, on a $F^{*}(0)=0$;

l) si $\overline{\overline{\boldsymbol{F}(\boldsymbol{X})}} \leqslant \mathfrak{f}(\overline{\overline{\boldsymbol{X}}})$, resp. $\overline{\overline{\boldsymbol{F}(\boldsymbol{X})}} \geqslant \mathbf{f}(\overline{\overline{\boldsymbol{X}}})$ pour toute classe d'ensembles $\boldsymbol{X}$, on a aussi $\left.\overline{\overline{\boldsymbol{H}^{*}(\boldsymbol{\boldsymbol { X }}}}\right) \leqslant \mathrm{f}(\overline{\overline{\boldsymbol{X}}})$, resp. $\left.\overline{\overline{\boldsymbol{F}^{*}(\boldsymbol{X}}}\right) \geqslant \mathrm{f}(\overline{\overline{\boldsymbol{X}})}$ pour toute classe $\boldsymbol{X}$.

Démonstration ne prête pas de difficulté; on s'appuie sur les définitions 15 et antérieures et sur le lem. 18.

Comme il est facile de voir, la signification du symbole $F^{*}$ dépend non seulement de celle de $\boldsymbol{F}$, mais aussi, tout comme dans le cas du symbole $C$, de l'interprétation du signe 1 (cf. p. 183). Si l'on voulait supprimer cette dépendance de l'opération $F^{*}$ de la signification de 1 , il faudrait convenablement transformer les déf. 14 et 15 , en posant p. ex. $\boldsymbol{C}_{A}(\boldsymbol{K})=E\left[X_{\epsilon} \boldsymbol{K}\right]$ et $\boldsymbol{F}^{\times}(\boldsymbol{K})=\boldsymbol{C}_{\boldsymbol{\Sigma}(\boldsymbol{K})} \boldsymbol{F} \boldsymbol{C}_{\boldsymbol{\Sigma}(\boldsymbol{K})}^{(\boldsymbol{K}) \text {. La }}$ notion d'opération double ainsi modifiée ne coinncide plus avec la notion primitive et perd plusieurs propriétés précienses an point de vue de ces recherches (en particulier le th. 33 du $\&$ suivant cesse d'être valable). Il existe tontefois un nombre d'opératlons $\boldsymbol{F}$ dont les deux opérations doubles $\boldsymbol{F}^{*}$ et $\boldsymbol{F}^{\times}$coincident, de sorte que l'opération $\boldsymbol{F}^{*}$ conserve alors sa signification unique indépendamment du mode de l'interprétation du signe 1; telles sont en particulier les opérations qui feront l'objet des $\$ \S 5$ et 6 de cet ouvrage.

\section{\$ 3. Notion générale de classe close par rapport à une opération donnée.}

Dans ce $\S$ je me propose d'examiner les propriétés générales des classes closes par rapport aux opérations quelconques.

Une classe d'ensembles $\boldsymbol{K}$ est dite close par rapport à une opération $\boldsymbol{F}$ donnée, lorsque $\boldsymbol{F}(\boldsymbol{K}) \subset \boldsymbol{K}$.

On pourrait en outre distingner les classes closes au sens plus étroit, c.-à-d. vérifiant la formule: $\overline{\boldsymbol{F}} \boldsymbol{U}(\boldsymbol{K}) \subset \boldsymbol{U}(\boldsymbol{K})$. Cépendant cette distinetion est dépourvae d'importance ici, les deux notions coïncidant pour les opérations monotones et toutes les opérations qui nous intéressent dans ce travail étant de cette sorte. 
Je vais introduire un signe spécial pour désigner la famille de toutes les classes closes par rapport à une opération $\boldsymbol{F}$ :

Définition 16. $\mathscr{C}\left(\boldsymbol{F}^{\prime}\right)=\underset{\boldsymbol{X}}{E}[\boldsymbol{F}(\boldsymbol{X}) \subset \boldsymbol{X}]$.

J'admets, comme toujours, que les classes de la famille $\mathfrak{C}_{h}\left(\boldsymbol{F}^{\prime}\right)$ se composent exclusivement de sous-ensembles d'un ensemble 1 donné auparavant (bien que la relativité de la notion envisagée envers l'ensemble 1 n'est pas mise en évidence dans le symbole $\mathscr{Q}\left(\boldsymbol{F}^{\prime}\right)$, comme il a été d'ailleurs anoncé au début du $\S 1$ ). En tenant compte de ce fait, on peut établir facilement le

Théorème 20. F étant une opération arbitraire, on a $U(1) \in \mathcal{E}(\mathbb{F})$ et $\mathcal{Q}\left(\boldsymbol{F}^{\prime}\right) \subset \boldsymbol{U} \boldsymbol{U}(1)$.

Démonstration. Suivant la déf. $5^{\mathrm{a}}, \boldsymbol{U}(1)$ est la classe de tous les ensembles possibles (contenus dans 1) et $\boldsymbol{U} V(1)$ est la famille de toutes les classes possibles (contenues dans $U(1)$ ). Or, comme $F(U(1))$ est également une classe d'ensembles, on a $F(U(1)) C$ $\subset \boldsymbol{U}(1)$, d'où, en raison de la déf. 16, $U(1) \in \mathcal{Q} \mathscr{\ell}\left(\boldsymbol{F}^{\prime}\right)$; de plus, $\mathcal{Q}\left(\boldsymbol{F}^{\prime}\right)$ étant une famille de classes, on a $\mathscr{C}\left(\boldsymbol{F}^{\prime}\right) \subset U U(1)$, c. q. f. d.

Théorème 21. a) $S i \mathcal{F}^{\circ} \boldsymbol{G}$, on a $\mathscr{C}(G) \subset \mathcal{E} \mathcal{L}\left(\boldsymbol{F}^{\prime}\right)$;

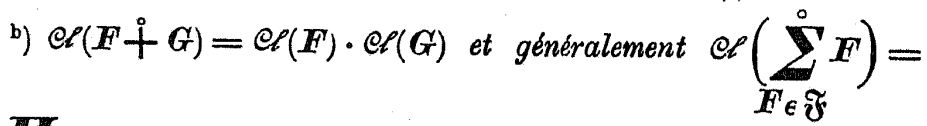
$=\prod_{\mathscr{F}} \mathcal{Q R}\left(F^{\prime}\right)$

Démonstration résulte facilement des déf. $8^{b}, 9$ et 16 .

M'appayant sur le th. $21^{\text {b }}$, je vais employer constamment les symboles $\mathscr{C}(\boldsymbol{F}+G)$ et $\mathscr{C}\left(\sum_{\boldsymbol{F} \in \mathfrak{F}} \boldsymbol{F}^{0}\right)$ pour désigner les familles de toutes les classes closes respectivement: par rapport aux deux opérations $F$ et $\mathfrak{G}$ à la fois, ou par rapport à toutes les opérations d'une classe $\mathfrak{F}$
donnée simultanément.

Le th. $21^{\mathrm{b}}$ implique immédiatement le

Corollaire 22. Si $\mathscr{C}(\boldsymbol{F})=\mathscr{C} \mathcal{C}(\boldsymbol{G})$, on a $\mathscr{C}(\boldsymbol{F}+\boldsymbol{H})=\mathscr{C}(\boldsymbol{G}+\boldsymbol{H})$
Théorème 23. ") $\mathscr{C}(I)=U \boldsymbol{U}(1)$;

b) $\mathscr{e}\left(I+\boldsymbol{H}^{\prime}\right)=\mathscr{e}(\boldsymbol{F})$;

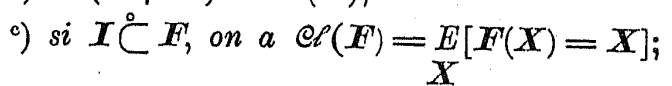

d) st $\boldsymbol{H}^{\mathrm{Q}} \stackrel{\circ}{=} \boldsymbol{F}$ et $\boldsymbol{I} \subset^{\circ} \boldsymbol{F}$, on a $\mathscr{C}(\boldsymbol{F})=\overline{\boldsymbol{F}} \boldsymbol{U} \boldsymbol{U}(1)$.

Démonstration. a) résulte aussitôt des déf. 16 et 13 .

b) En vertu du th. $21^{\text {b }}, \mathscr{e}\left(I+\boldsymbol{I}^{\prime}\right)=\mathscr{C} \mathcal{C}(\boldsymbol{I}) \cdot \mathscr{C}\left(\boldsymbol{F}^{\prime}\right)$; comme, en raison de $\left.{ }^{a}\right)$ et du th. $20, \mathfrak{e} \ell\left(\boldsymbol{F}^{\prime}\right) \subset \mathscr{C}(\boldsymbol{I})$, on en obtient: $\mathscr{C}\left(\boldsymbol{I}+\boldsymbol{F}^{\prime}\right)=$ $=\mathscr{O}\left(\boldsymbol{H}^{\prime}\right)$, c. q. f. d.

c) est une conséquence immédiate de la déf. 16 et du lem. $17^{\text {f }}$.

d) $\boldsymbol{U} \boldsymbol{U}(1)$ étant la famille de toutes les classes d'ensembles, on conclut de $\left.{ }^{c}\right)$ que

$$
\mathscr{e}(\boldsymbol{F}) \subset \underset{\boldsymbol{F}(\boldsymbol{X})}{E}\left[\boldsymbol{X}_{\epsilon} \boldsymbol{U} \boldsymbol{U}(\boldsymbol{I})\right]
$$

D'autre part, envisageons une classe arbitraire d'ensembles $\boldsymbol{X}$ $\left(\boldsymbol{X}_{\epsilon} \boldsymbol{U U}(1)\right)$ et posons: $\boldsymbol{Y}=\boldsymbol{F}(\boldsymbol{X})$. Comme $\boldsymbol{F}^{2} \stackrel{\circ}{=} \boldsymbol{F}$, on a suivant les déf. $8^{\mathrm{a}}$ et 10: $\boldsymbol{F}(\boldsymbol{Y})=\boldsymbol{F} \boldsymbol{F}(\boldsymbol{X})=\mathbb{F}^{\mathbf{a}}(\boldsymbol{X})=\boldsymbol{F}(\boldsymbol{X})=Y$, d'où à fortiori $\boldsymbol{F}(\boldsymbol{Y}) \subset \boldsymbol{Y}$, donc, conformément à la déf. 16, $\boldsymbol{Y}_{\epsilon} \mathfrak{Q} \mathcal{L}\left(\boldsymbol{F}^{\prime}\right)$. Il est ainsi démontré que pour tout $\boldsymbol{X}_{\epsilon} \boldsymbol{U U}(1)$ on a $\boldsymbol{F}(\boldsymbol{X}) \epsilon \mathcal{C}(\boldsymbol{F})$; en d'autres termes

$$
\underset{F(\boldsymbol{X})}{E}\left[\boldsymbol{X}_{\epsilon} \boldsymbol{U} \boldsymbol{U}(\boldsymbol{1})\right] \subset \mathcal{C} \ell\left(\boldsymbol{F}^{\prime}\right)
$$

Les inclusions (1) et (2) donnent l'égalité: $\mathcal{Q}\left(\boldsymbol{F}^{\prime}\right)=E\left[\boldsymbol{X}_{\epsilon} \boldsymbol{V} \boldsymbol{V}(1)\right.$; en posant dans la déf. $6: f \stackrel{\circ}{=}$ et $A=\boldsymbol{U} \boldsymbol{U}(1)$, on en obtient la formule cherchée:

$$
\mathscr{Q}(\boldsymbol{F})=\overline{\boldsymbol{F}} \boldsymbol{U} \boldsymbol{U}(1)
$$

En' raison du th. $23^{\mathrm{b}}$ il est facile de comprendre pourquoi on peut se borner ici aux opérations $G$ adjonctives, c.-à-d. vérifiant l'inclusion: $\boldsymbol{I} \subset^{0} \boldsymbol{G}$ (cf. p. 209). En exiaminant la notion de classe close par rapport à une opération arbitraire $\boldsymbol{F}$, on peut en effet remplacer cette opération par une opération adjonctive $G$ (notamment par $G \doteq I+F$ ) de façon que les familles correspondantes $\mathscr{C}(\boldsymbol{F})$ et $\mathfrak{Q}(\boldsymbol{G})$ coïncident.

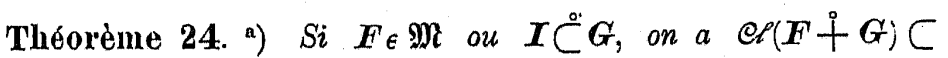
$\mathcal{C} \mathcal{P}(\boldsymbol{F} \boldsymbol{G})$ 


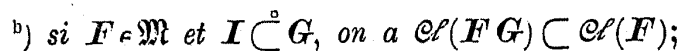

c) si $\boldsymbol{I} \dot{C}^{\circ} \boldsymbol{F}$, on a $\mathscr{e}(\boldsymbol{F} \boldsymbol{G}) \subset \mathscr{C}(\boldsymbol{G})$;

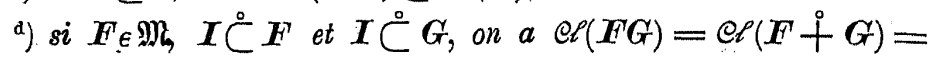
$=\mathscr{Q}\left(\boldsymbol{F}^{\prime}\right) \cdot \mathscr{Q} \mathcal{L}\left(\boldsymbol{G}_{\mathrm{r}}\right)$

Démonstration. a) Soit $\boldsymbol{X}_{\boldsymbol{\epsilon}} \mathcal{C} \mathcal{P}(\boldsymbol{F}+\boldsymbol{G}) \cdot$ En raison des déf. 16 et $9^{a}$, on a $\boldsymbol{F}(\boldsymbol{X})+\boldsymbol{G}(\boldsymbol{X}) \subset \boldsymbol{X}$, d'où $\boldsymbol{F}(\boldsymbol{X}) \subset \boldsymbol{X}$ et $\boldsymbol{G}(\boldsymbol{X}) \subset \boldsymbol{X}$. Si $F_{\mathcal{F}} \mathfrak{M}$, on applique le lem. $15^{\mathrm{a}}$, en y remplaçant $\boldsymbol{X}$ par $\boldsymbol{G}(\boldsymbol{X})$ et $\boldsymbol{Y}$ par $\boldsymbol{X}$; de l'inclusion: $\boldsymbol{G}(\boldsymbol{X}) \subset \boldsymbol{X}$ on obtient ainsi: $\boldsymbol{F} \boldsymbol{G}(\boldsymbol{X}) \subset$ $\subset \boldsymbol{F}(\boldsymbol{X})$, ce qui, rapproché de la formule: $\boldsymbol{F}(\boldsymbol{X}) \subset \boldsymbol{X}$, donne: $\boldsymbol{F} \boldsymbol{G}(\boldsymbol{X}) \subset \boldsymbol{X}$. Si, d'autre part, $\boldsymbol{I} \subset^{\circ} \boldsymbol{G}$, on a en vertu du lem. $17^{\circ}$ $\boldsymbol{X} \subset \boldsymbol{G}(\boldsymbol{X})$, d'où $\boldsymbol{G}(\boldsymbol{X})=\boldsymbol{X}$; en remplaçant donc $\boldsymbol{X}$ par $\boldsymbol{G}(\boldsymbol{X})$ dans la formule: $\boldsymbol{F}(\boldsymbol{X}) \subset \boldsymbol{X}$, on obtient de nouveau: $\boldsymbol{F} \boldsymbol{G}(\boldsymbol{X}) \subset \boldsymbol{X}$. Or, conformément à la déf. 16, cette dernière inclusion équivaut à la formule: $\boldsymbol{X}_{\boldsymbol{\epsilon}} \mathfrak{Q} \mathcal{Q}(\boldsymbol{F} G)$.

Nous avons ainsi prouvé que dans les hypothèses du lemme la formule: $\boldsymbol{X}_{\epsilon} \mathscr{C}(\boldsymbol{F}+\boldsymbol{G})$ entraîne toujours: $\boldsymbol{X}_{\epsilon} \mathscr{e}(\boldsymbol{F} \boldsymbol{G})$; par conséquent l'inclusion cherchée: $\mathscr{C}(\boldsymbol{F}+\boldsymbol{G}) \subset \mathscr{C}(\boldsymbol{F} \boldsymbol{G})$ se trouve établie.

b) Suivant le lem. $17^{\circ}$ les formules $\boldsymbol{F} \in \mathfrak{M}$ et $I \subset^{\circ} G$ donnent $\boldsymbol{F} \complement^{\circ} \boldsymbol{F G}$; à l'aide du th. $21^{\mathrm{a}}$ on en conclut que $\mathscr{C}(\boldsymbol{F} \boldsymbol{G}) \subset \mathcal{Q} \mathcal{P}(\boldsymbol{F})$, c. q. f. $d$.

c) se démontre d'une façon complètement analogue.

d) Les formules aj-c) établies tout à l'heure impliquent que $\mathscr{e}(\boldsymbol{F}+\boldsymbol{G}) \subset \mathscr{C}(\boldsymbol{F} \boldsymbol{G}) \subset \mathscr{e}(\boldsymbol{F}) \cdot \mathscr{C}(\boldsymbol{G})$. En rapprochant cette inclusion double du th. $21^{\mathrm{b}}$ on en obtient aussitôt:

$$
\mathscr{Q}(\boldsymbol{F} \boldsymbol{G})=\mathscr{e}(\boldsymbol{F}+\boldsymbol{G})=\mathscr{C}(\boldsymbol{F}) \cdot \mathscr{Q}(\boldsymbol{G}) \text {, c. q. f. d. }
$$

Théorème 25. Si $I \complement^{\circ} F^{\prime}$ et $1 \leqslant v<\omega$, on a $\mathscr{C}\left(\boldsymbol{F}^{\nu}\right)=\mathscr{O} \mathcal{L}\left(\boldsymbol{F}^{\prime}\right)$.

Démonstration. On déduit facilement de la déf. 10 les formules suivantes:

$$
\text { (1) } \boldsymbol{F}^{\mathbf{1}} \stackrel{\circ}{=} \boldsymbol{F}^{\prime} \text { et } \boldsymbol{F}^{\nu} \boldsymbol{F}^{\circ} \stackrel{\circ}{=} \boldsymbol{F} \boldsymbol{F}^{\nu} \stackrel{\circ}{=} \boldsymbol{F}^{\nu+1}(\text { pour } 1 \leqslant \nu<\omega) \text {. }
$$

En remplaçant dans les th. $21^{\mathrm{b}}$ et $24^{\mathrm{a}} \boldsymbol{F}$ par $\boldsymbol{F}^{\nu}$ et $G$ par $\boldsymbol{F}$, on obtient: $\mathscr{C}\left(\boldsymbol{F}^{\nu}\right) \cdot \mathscr{C}\left(\boldsymbol{F}^{\prime}\right)=\mathscr{C}\left(\boldsymbol{F}^{\nu}+\boldsymbol{F}^{\prime}\right) \subset \mathscr{C}\left(\boldsymbol{H}^{\nu} \boldsymbol{W}\right)$; d'où selon (1)

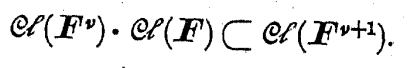

A l'aide du principe de l'induction complète on en conclut que (3)

$$
\mathscr{Q}\left(\boldsymbol{F}^{\prime}\right) \subset \mathcal{Q} \mathcal{L}\left(\boldsymbol{F}^{\nu}\right) \quad(\text { pour } 1 \leqslant \nu<\omega) .
$$

En effet, le nombre $\nu=1$ vérifie la formule (3), puisqu'on a suivant (1) $\mathscr{C}\left(\boldsymbol{F}^{\prime}\right)=\mathscr{C} \mathcal{\ell}\left(\boldsymbol{F}^{1}\right)$; si ensuite un nombre arbitraire $\boldsymbol{\nu}$, où $1 \leqslant \nu<\omega$, remplit la formule $(3)$, on obtient, d'après $(4), \mathcal{e} \ell(F) \subset$ $\subset \mathcal{Q}\left(\boldsymbol{F}^{\nu+1}\right)$, ce qui veut dire que le nombre $\nu+1$ remplit la même formule.

D'autre part, en posant dans le th. $24^{\mathrm{c}}: G \stackrel{\circ}{=} F^{\nu}$ et 'en tenant compte de (1), on parvient à la formule: $\mathscr{e}\left(\boldsymbol{F}^{\nu+1}\right) \subset \mathcal{C}\left(\boldsymbol{F}^{\nu}\right)$, d'où on obtient par une induction facile:

$$
\mathscr{Q}\left(\boldsymbol{F}^{\nu}\right) \subset \mathcal{Q} P\left(\boldsymbol{F}^{11}\right)=\mathscr{Q}(\boldsymbol{F}) \quad(\text { pour } 1 \leqslant \nu<\omega) .
$$

Les inclusions (3) et (4) donnent aussitôt:

$$
\mathscr{Q}\left(\boldsymbol{F}^{\nu}\right)=\mathscr{Q} \mathcal{C}(\boldsymbol{F}), \text { c. q. f. d. }
$$

Théorème 26. $\mathscr{C}\left(\boldsymbol{F}^{*}\right)=\dot{\overline{\boldsymbol{C}}}\left(\mathcal{C}\left(\boldsymbol{F}^{\prime}\right)\right)$.

Démonstration. Soit $\boldsymbol{X}_{\boldsymbol{\epsilon}} \mathfrak{E}\left(\boldsymbol{F}^{\prime}\right)$, donc d'après la déf. 16 $\boldsymbol{F}(\boldsymbol{X}) \subset \boldsymbol{X}$. A l'aide des lem. $15^{\mathrm{a}}$ et $18^{\mathrm{a}}$ on en obtient: $\boldsymbol{C F}(\boldsymbol{X}) \subset$ $\subset \boldsymbol{C}(\boldsymbol{X})$, d'où en vertu du lem. 19a $\boldsymbol{F}^{*} \boldsymbol{C}(\boldsymbol{X}) \subset \boldsymbol{C}(\boldsymbol{X})$. Par conséquent, en raison de la déf. 16 , on a $C(X) \in \mathcal{Q} \ell\left(\boldsymbol{F}^{*}\right)$.

Ce raisonnement prouve l'inclusion: $\overline{\boldsymbol{C}}(\mathcal{Q} \ell(\boldsymbol{F})) \subset \mathcal{Q P}\left(\boldsymbol{F}^{*}\right)$.

D'une façon tout à fait analogue on peut démontrer qu'à toute classe $\boldsymbol{Y}_{\epsilon} \mathfrak{Q} \mathcal{P}\left(\boldsymbol{F}^{*}\right)$ correspond une classe $\boldsymbol{X}_{\boldsymbol{E}} \mathcal{Q P}(\boldsymbol{F})$ telle que $\boldsymbol{Y}=\boldsymbol{C}(\boldsymbol{X})$. Il en résulte l'inclusion: $\mathfrak{Q}\left(\boldsymbol{F}^{*}\right) \subset \overline{\boldsymbol{C}}(\mathcal{Q}(\boldsymbol{F}))$, qui, rapprochée de l'inclusion précédente, donne lidentité cherchée:

$$
\mathscr{Q}\left(\boldsymbol{F}^{*}\right)=\overline{\boldsymbol{C}}(\mathcal{Q} \mathcal{R}(\boldsymbol{F}))
$$

Corollaire 27. Si $\mathscr{C}(\boldsymbol{F})=\mathscr{C}(\boldsymbol{G})$, on a $\mathscr{C}\left(\boldsymbol{F}^{*}\right)=\mathscr{C}\left(\boldsymbol{G}^{*}\right)$.

Théorème 28, a) Si $\boldsymbol{F} \in \mathfrak{M}$ et $\boldsymbol{I} \subset \boldsymbol{F}$, on a $\quad \mathcal{Q}(\boldsymbol{C}+\boldsymbol{F})=$

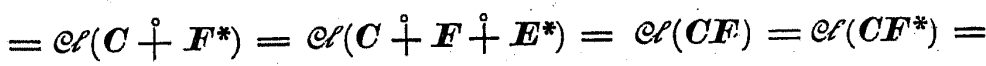
$=\mathfrak{Q}\left(\boldsymbol{C F} F^{*}\right)$;

b) $8 i \boldsymbol{F} \in \mathfrak{M}$, on a $\mathscr{C} \ell(\boldsymbol{C}+\boldsymbol{F})=\mathcal{C} \ell\left(\boldsymbol{C} \dot{+} \boldsymbol{F}^{*}\right)=\mathscr{C}\left(\boldsymbol{C}+\boldsymbol{F}+\boldsymbol{F}^{*}\right)$.

Démonstration. a) En vertu du lem. 19g, on a par hypothèse 
à l'aide des lem. $16^{\mathrm{c}}, 17^{\mathrm{b}}$ et $18^{\mathrm{a}}$ on obtient de l'hypothèse et de (1):

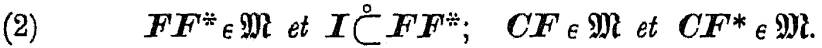

En appliquant à deux reprises le th. $24^{\text {a }}$ (pour $\boldsymbol{F} \stackrel{\circ}{=} \boldsymbol{C}$ et $\boldsymbol{G}=\boldsymbol{F}$ et puis pour $\boldsymbol{F} \stackrel{\circ}{=} \boldsymbol{C}$ et $G \stackrel{\circ}{=} \boldsymbol{F}^{*}$ ) et en tenant compte du lem. $18^{\mathrm{a}}$, on conclat que

(3) $\quad \mathcal{Q}(\boldsymbol{C}+\boldsymbol{F}) \subset \mathcal{O} \mathcal{P}(\boldsymbol{C F})$ et $\mathscr{Q}\left(\boldsymbol{C}+\boldsymbol{F}^{*}\right) \subset \mathcal{Q} \mathcal{P}\left(\boldsymbol{C F ^ { * }}\right)$;

tout pareillement, en vertu de l'hypothèse et de $(1)$, le th. $24^{\mathrm{b}}$ donne:

$$
\mathcal{Q}(\boldsymbol{C F}) \subset \mathcal{Q}(\boldsymbol{C}) \text { et } \mathscr{e}\left(\boldsymbol{C \boldsymbol { F } ^ { * }}\right) \subset \mathcal{e}(\boldsymbol{C}) \text {. }
$$

En remplaçant dans le th. $24^{\text {a }} \boldsymbol{F}$ et $G$ par $C F$, resp. par

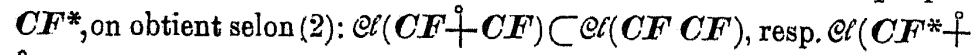
$\left.+\boldsymbol{C} \boldsymbol{F}^{*}\right) \subset \mathcal{e}\left(\boldsymbol{C \boldsymbol { F } ^ { * }} \boldsymbol{C \boldsymbol { F } ^ { * }}\right)$, d'où en raison du lemme $19^{\mathrm{a}, \mathrm{b}}$

$$
\mathfrak{Q}(\boldsymbol{C F}) \subset \mathfrak{e}\left(\boldsymbol{F}^{*} \boldsymbol{F}\right) \text { et } \mathfrak{e} l\left(\boldsymbol{C \boldsymbol { E } ^ { * }}\right) \subset \mathfrak{e}\left(\boldsymbol{F} \boldsymbol{F}^{*}\right) \text {. }
$$

En tenant compte de l'hypothèse et de (1), le th. $24^{\mathrm{b}}$ implique encore que $\mathfrak{C l}\left(\boldsymbol{F}^{*} \boldsymbol{F}\right) \subset \mathfrak{C} \mathfrak{C}\left(\boldsymbol{F}^{*}\right)$ et $\mathfrak{C}\left(\boldsymbol{F}^{*}\right) \subset \mathfrak{C}\left(\boldsymbol{F}^{\prime}\right)$; en rapprochant ces inclusions de (5), nous obtenons:

$$
\mathfrak{e}(\boldsymbol{C F}) \subset \mathfrak{e}\left(\boldsymbol{F}^{*}\right) \text { et } \mathfrak{e}\left(\boldsymbol{C \boldsymbol { F } ^ { * } )} \subset \mathfrak{e}(\boldsymbol{F})\right. \text {. }
$$

Les formules (4) et (6) donnent: $\mathfrak{C}(\boldsymbol{C} \boldsymbol{F}) \subset \mathfrak{e}(\boldsymbol{C}) \cdot \mathfrak{C} \ell\left(\boldsymbol{F}^{*}\right)$ et $\mathfrak{C}\left(\boldsymbol{C F ^ { * }}\right) \subset \mathfrak{e}(\boldsymbol{C}) \cdot \mathfrak{e}(\boldsymbol{F})$; comme, en vertu du th. $21^{\mathrm{b}}$,

$$
\mathfrak{C}(\boldsymbol{C}) \cdot \mathfrak{C}\left(\boldsymbol{F}^{*}\right)=\mathfrak{e}\left(\boldsymbol{C}+\boldsymbol{F}^{*}\right) \text { et } \mathfrak{C}(\boldsymbol{C}) \cdot \mathfrak{C} l(\boldsymbol{F})=\mathfrak{C} l(\boldsymbol{C}+\boldsymbol{F}),
$$

on en conclut que

$$
\mathfrak{C}(\boldsymbol{C F}) \subset \mathfrak{e}\left(\boldsymbol{C}+\boldsymbol{F}^{*}\right) \text { et } \mathfrak{e}\left(\boldsymbol{C \boldsymbol { F } ^ { * } )} \subset \mathfrak{e}(\boldsymbol{C}+\boldsymbol{F}) .\right.
$$

De (3) et (7) on déduit aussitôt:

$$
\mathscr{C}(\boldsymbol{C}+\boldsymbol{F})=\mathfrak{e}\left(\boldsymbol{C}+\boldsymbol{F}^{*}\right)=\mathfrak{e}(\boldsymbol{C F})=\mathfrak{e}\left(\boldsymbol{C \boldsymbol { F } ^ { * }}\right) .
$$

Le cor. 22, si l'on y remplace $\boldsymbol{F}$ par $\boldsymbol{C}+\boldsymbol{F}, \boldsymbol{G}$ par $\boldsymbol{C}+\boldsymbol{F}^{*}$ et $\boldsymbol{H}$ par $\boldsymbol{F}^{*}$, entraîne selon (8):

$$
\mathfrak{e}\left(\boldsymbol{C}+\boldsymbol{F} \stackrel{+}{+\boldsymbol{F}^{*}}\right)=\mathfrak{e} \ell\left(\boldsymbol{C}+\boldsymbol{F}^{*}\right) .
$$

Appliquons enfin le th. $24^{\text {d }}$, en y posant: $G^{\circ}=F^{*}$; en raison de l'hypothèse et de (1), nous en concluons que $\mathfrak{e} \ell\left(\boldsymbol{F F}^{*}\right)=\mathcal{e} \rho\left(\boldsymbol{F}+\boldsymbol{F}^{*}\right)$ d'où suivant le cor. 22

$$
\mathfrak{e}\left(\boldsymbol{C}+\boldsymbol{F}^{*} \boldsymbol{F}^{*}\right)=\mathfrak{e} l\left(\boldsymbol{C}+\boldsymbol{F}+\boldsymbol{F}^{*}\right) .
$$

La formule (8) ayant été déduite pour to u te opération monotone et adjonctive, on peut y remplacer selon (2) en particulier $\boldsymbol{F}$ par $\boldsymbol{E F}^{*}$; on obtient donc: $\mathfrak{C}\left(\boldsymbol{C}+\boldsymbol{F} \boldsymbol{F}^{* *}\right)=\mathfrak{E} C\left(\boldsymbol{C F} \boldsymbol{F}^{*}\right)$, d'où en verta de (10)

$$
\mathfrak{e} C\left(\boldsymbol{C}+\boldsymbol{F}^{\circ} \boldsymbol{F}^{*}\right)=\mathfrak{Q} \ell\left(\boldsymbol{C F} \boldsymbol{F}^{*}\right)
$$

Les égalités (8), (9) et (11) constituent la formule à démontrer.

b) En vertu des lem. $16^{c}, 17^{\mathrm{a}}$ et 13 on a $\boldsymbol{I} \dot{+} \boldsymbol{F} \in \mathfrak{M}$ et $\boldsymbol{I} \dot{C} \boldsymbol{I}+\boldsymbol{F}$, d'où, en remplaçant dans a) $\boldsymbol{F}$ par $\boldsymbol{I}+\boldsymbol{F}$ et en tenant compte du lem. $19^{\mathrm{d}, 1}$, on obtient: $\mathscr{C}(\boldsymbol{C}+\boldsymbol{I}+\boldsymbol{F})=\mathfrak{C} C\left(\boldsymbol{C}+\boldsymbol{I} \stackrel{+}{+} \boldsymbol{F}^{*}\right)=\mathfrak{e}_{\ell}(\boldsymbol{C}+$ $\left.+\boldsymbol{I}+\boldsymbol{F}+\boldsymbol{F}^{*}\right)$. La formule cherchée en résulte aussitôt selon le th. $23^{\mathrm{b}}$.

On peut déduire des th. 24 et 28 certaines conséquences d'un caractère plus général. Considérons une classe arbitraire $\mathfrak{F}$ composée exclusivement d'opérations monotones et adjonctives et ajoutons à cette classe, si l'on veut, l'opération $C$, qui est aussi monotone, mais pas adjonctive. A l'aide de l'addition et de la multiplication relative on peut former des opérations de la classe $\mathfrak{F}$, resp. $\{\boldsymbol{C}\}+\mathfrak{F}$, diverses autres opérations; nous avons convenu de désigner par le symbole $\mathfrak{B}(\mathfrak{F})$, resp. $\mathfrak{B}(\{\boldsymbol{C}\}+\widetilde{f})$, la classe de toutes les opérations ainsi formées (cf. la déf. $\left.11^{c}\right)$. Or, en généralisant les résultats exprimés par les th. $24^{\mathrm{d}}$ et $28^{\mathrm{a}}$, on pent montrer qu'au cours de l'étude des classes closes par rapport aux opérations considérées on peut se restreindre à n'envisager que les opérations de stractare asser simple, à savoir celles qui s'obtiennent des opérations de la classe primitive $\widetilde{f}$, de leurs opérations doubles et de deux opérations $\boldsymbol{C}$ et $\boldsymbol{I}$ par la seule addition. En symboles introduits dans la déf. 11, on peut démontrer notamment les théorèmes suivants:

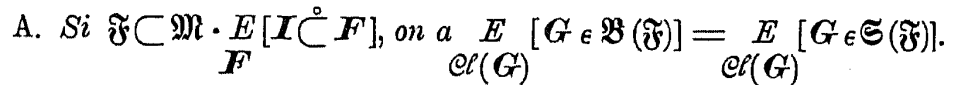

B. Si $\mathfrak{\mho} \subset \mathfrak{M} \cdot \underset{\boldsymbol{F}}{E}\left[\boldsymbol{I} \subset \mathcal{C}^{\circ}\right]$ et $\mathfrak{H}=\underset{H^{*}}{E}[\boldsymbol{F} \in \mathfrak{\mho}]$, on a

$\underset{\mathfrak{e} \ell(\boldsymbol{G})}{E}[\boldsymbol{G} \in \mathfrak{B}(\{\boldsymbol{C}\}+\mathfrak{F})]=\underset{\mathfrak{C}(\boldsymbol{G})}{E}[\boldsymbol{G} \in \mathfrak{S}\{\{\boldsymbol{I}, \boldsymbol{C}\}+\mathfrak{F}+\mathfrak{( \mathfrak { S } )}]=$ $=\{\mathfrak{C}(\boldsymbol{I}), \mathfrak{e}(\boldsymbol{C})\}+\underset{\mathfrak{C}(\boldsymbol{C}+\boldsymbol{G})}{E}[\boldsymbol{G} \in \mathcal{S}(\mathfrak{F})]+\underset{\mathfrak{C} \ell(\boldsymbol{G})}{E}[\boldsymbol{G} \in \mathfrak{S}(\mathfrak{F}+(\mathfrak{S})]$.

Dans le cas particalier où la classe $\mathfrak{\mho}$ se compose d'une seule opération $\boldsymbol{F}$, les th. A et $\mathrm{B}$ donnent les corollaires suivants:

C. Si $\boldsymbol{F} \in \mathfrak{M}$ et $\boldsymbol{I} \complement^{\circ} \boldsymbol{F}$, on a $\underset{\mathfrak{C C}(\boldsymbol{G})}{E}[\boldsymbol{G} \in \boldsymbol{B}(\{\boldsymbol{F}\rangle)]=\{\mathfrak{e l}(\boldsymbol{F})\}$.

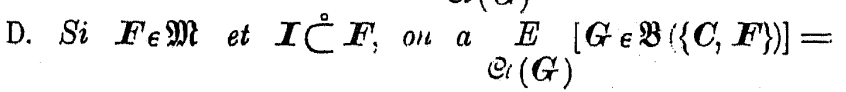
$=\left\{\mathfrak{e l}(\boldsymbol{I}), \mathfrak{C} \ell(\boldsymbol{C}), \mathfrak{e}\left(\boldsymbol{F}^{\prime}\right), \mathfrak{e}\left(\boldsymbol{F}^{*}\right), \mathfrak{e} l(\boldsymbol{C} \stackrel{+}{\boldsymbol{F}}), \mathfrak{e}\left(\boldsymbol{F}^{\circ}+\boldsymbol{F}^{*}\right)\right\}$. 
On peut en outre supprimer dans le cor. C l'hypothèse: $\boldsymbol{F} \in \mathfrak{M}$, en obtenant ainsi une généralisation du th. 25 . En se bornant aux opérations de la classe $\mathfrak{P}\left(\{\boldsymbol{C}, \boldsymbol{F}\}\right.$ ) (cf. la déf. $11^{\mathrm{b}}$ ), le cor. D peut être développé et complété comme suit:

$$
\begin{aligned}
& \text { E. a) } \mathfrak{B}(\{C, F\})=\{\boldsymbol{I}, \boldsymbol{C}\}+\mathfrak{P}\left(\left\{\boldsymbol{F}, \boldsymbol{F}^{*}\right\}\right)+\underset{\boldsymbol{C} \boldsymbol{G}}{\boldsymbol{E}}\left[\boldsymbol{G} \in \mathfrak{P}\left(\left\{\boldsymbol{F}, \boldsymbol{F}^{*}\right\}\right)\right] \text {; } \\
& \text { b) si } \boldsymbol{F} \in \mathfrak{M} \text { et } \boldsymbol{I} \stackrel{\circ}{\complement} \boldsymbol{F} \text {, on a } \\
& \stackrel{E}{\mathscr{P}(G)} \\
& \underset{E}{E}\left[\boldsymbol{G} \in \mathfrak{B}\left(\left\{\boldsymbol{F}, \boldsymbol{F}^{*}\right\}\right]=\left\{\mathfrak{Q} \mathcal{P}\left(\boldsymbol{F}^{\prime}\right), \mathfrak{e} \mathcal{P}\left(\boldsymbol{F}^{*}\right), \mathfrak{e}\left(\boldsymbol{F}+\boldsymbol{F}^{*}\right)\right\}\right. \text { et } \\
& \underset{\mathcal{C}_{\ell}(\boldsymbol{C G})}{E}\left[\boldsymbol{G} \in \mathfrak{P}\left(\left\{\boldsymbol{F}, \boldsymbol{F}^{*}\right\}\right)\right]=(\mathfrak{C} \mathcal{P}(\boldsymbol{C}+\boldsymbol{F})\} .
\end{aligned}
$$

Je renonce à donner ici la démonstration de ces théorèmes.

Le théorème suivant mérite d'être cité par sọn intérêt spécial, bien qu'il n'aura pas d'application dans les considérations ultérieures:

Théorème 29. Si $\boldsymbol{F}_{\in} \mathfrak{M}$ et $\mathscr{A C C} \mathscr{C}(\boldsymbol{F})$, on a $\Pi(\mathscr{O C}) \in \mathcal{Q} l\left(\boldsymbol{F}^{\prime}\right)$.

Démonstration. Soit $\boldsymbol{X}_{\epsilon} \mathscr{H}$. On a alors notoirement $\Pi(\mathscr{H}) \subset X_{\text {; }}$ l'opération $\boldsymbol{F}$ étant monotone, on en conclut suivant le lem. $15^{\mathrm{a}}$ que $\boldsymbol{F} \Pi(\mathscr{H}) \subset \boldsymbol{F}(\boldsymbol{X})$. Or, ce ruisonnement étant': valable pour une classe arbitraire de $\mathscr{A}$, on en obtient la formule:

$$
\boldsymbol{F} I(\mathscr{A}) \subset \prod_{\boldsymbol{X}_{\in} \mathfrak{d} \mathcal{L}} \boldsymbol{F}(\boldsymbol{X})
$$

Comme d'autre part $\mathscr{K} \subset \mathcal{O} \mathcal{P}(\boldsymbol{F})$, on a conformément à la déf. 16:

$$
\prod_{\boldsymbol{X} \in \mathscr{d}} \boldsymbol{F}(\boldsymbol{X}) \subset \prod_{\boldsymbol{X}_{\epsilon \mathscr{H}}} \boldsymbol{X}=\Pi(\mathscr{A} i)
$$

Les formules (1) et (2) donnent aussitôt: $\boldsymbol{F} \Pi(\mathscr{H}) \subset \Pi(\mathscr{A})$, d'où en raison de la déf. 16

$$
\Pi(\mathscr{d} i) \in \mathcal{E}(\boldsymbol{F}), \quad \text { c. q. f. d. }
$$

Corollaire 30. Si $\boldsymbol{F} \in \mathfrak{M}$, à toute classe d'ensembles $\boldsymbol{K}$ correspond une classe $\boldsymbol{L}$ vérifiant les formules: $\boldsymbol{L} \in \boldsymbol{V}(\boldsymbol{K}) \cdot \mathcal{O}\left(\boldsymbol{F}^{\prime}\right)$ et $\boldsymbol{P}(\boldsymbol{K}) \cdot \mathcal{e}(\boldsymbol{F}) \subset \boldsymbol{V}(\boldsymbol{L})$.

Démonstration. Posons: $\mathscr{H}=\boldsymbol{V}(\boldsymbol{K}) \cdot \mathcal{C} \mathscr{R}(\boldsymbol{F})$ et $L=I(\mathscr{H})$.

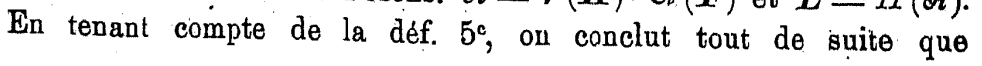

$\boldsymbol{L}_{\epsilon} \boldsymbol{V}(\boldsymbol{K})$ et que $\boldsymbol{V}(\boldsymbol{K}) \cdot \mathcal{C} \mathcal{P}(\boldsymbol{F}) \subset \boldsymbol{V}(\boldsymbol{L})$; de plus le th. 29 donne: $\boldsymbol{L} \in \mathcal{C} \mathcal{C}(\boldsymbol{F}) . \boldsymbol{L}$ est done la classe cherchée.

Il est à noter que l'hypothèse: $\boldsymbol{F}^{\prime} \in \mathfrak{M}$ joue dans les th. 29 et 30 un rôle essentiel; on pourrait néanmoins la supprimer, si l'on avait convenu de désigner par le symbole $\mathcal{C}\left(\boldsymbol{F}^{\prime}\right)$ la famille de toutes les classes closes au sens plus étroit par rapport à l'opération $\boldsymbol{F}^{\prime}$ (cf. p. 211).

Conformément au cor. 30, dans l'hypothèse que $\boldsymbol{F}$ est une opération monotone on peut faire correspondre à toute classe d'ensembles $\boldsymbol{K}$ la plus petite classe $\boldsymbol{L}$ contenant $\boldsymbol{K}$ et close par rapport à $\boldsymbol{F}$. Dans le cas le plus général on n'a que peu à dire sur la nature de cette classe. Ce n'est que dans les hypothèses spéciales que l'on peut établir quelques propriétés plus profondes de la classe $L$; on a p. ex. le théorème suivant:

Théorème 31. Si $\boldsymbol{F}_{\in} \mathfrak{X}_{\beta}$, ò̀ $c f(\beta)=\beta$, et si pour toute classe $\boldsymbol{X}$ on a $\overline{\overline{\boldsymbol{F}}(\overline{\boldsymbol{X}})} \leqslant(\overline{\overline{\boldsymbol{X}}})^{\aleph_{\beta}}$, alors à toute classe d'ensembles $\boldsymbol{K}$ telle que $\overline{\overline{\boldsymbol{K}}} \geqslant 2$ correspond une classe $\boldsymbol{L}$ vérifiant les formules:

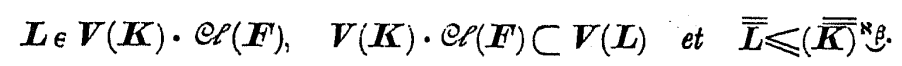

Démonstration. Définissons par recurrence une suite transfinie de classes d'ensembles $\boldsymbol{K}_{\xi}$ du type $\omega_{\beta}$, en posant

soit en outre

$$
\begin{gathered}
\boldsymbol{K}_{0}=\boldsymbol{K}, \\
\boldsymbol{K}_{\xi}=\boldsymbol{F}\left(\sum_{\eta<\xi} \boldsymbol{K}_{\eta}\right) \text { pour } 0<\xi<\omega_{\beta} ;
\end{gathered}
$$

$$
\boldsymbol{L}=\sum_{\xi<\omega_{\beta}} \boldsymbol{K}_{\xi} .
$$

Considérons un ensemble arbitraire $X$ tel que

$$
X \in \boldsymbol{F}^{\prime}(\boldsymbol{L}) \text {. }
$$

Comme par hypothèse $\boldsymbol{F} \in \mathfrak{A}_{\beta}$, le lem. $15^{\circ}$ donne: $\boldsymbol{F}(\boldsymbol{L})=$ $=\sum \boldsymbol{F}(\boldsymbol{Y})$; en vertu de (4) on en conclut qu'il existe une classe $\boldsymbol{Y}_{\boldsymbol{E}} \boldsymbol{U}_{\beta}(\boldsymbol{L})$

d'ensembles $\boldsymbol{Y}$ vérifiant les formules:

$X \in \boldsymbol{F}(\boldsymbol{Y})$ 
et $\boldsymbol{Y}_{\epsilon} \boldsymbol{U}_{\beta}(\boldsymbol{L})$ ou, en d'autres termes (cf. la déf. $5^{\mathrm{b}}$ ),

$$
Y \subset L \text { et } \overline{\bar{Y}}<s_{\beta} \text {. }
$$

En tenant compte de l'hypothèse et de (3), on obtient de (6): $\overline{\overline{\boldsymbol{Y}}}<\kappa_{c f(\beta)}$ et $\boldsymbol{Y} \subset \sum_{\xi<\omega_{\beta}} \boldsymbol{K}_{\bar{\xi}}$. Conformément au lem. $3^{\circ}$ (pour $B=\boldsymbol{Y}$ $\alpha=\beta$ et $F_{\xi}=\boldsymbol{K}_{\xi}$, ces formules impliquent l'existence d'un nombre ordinal $\xi$ tel que

$$
0<\xi<\omega_{\beta} \text { et } \quad \boldsymbol{Y} \subset \sum_{\eta<\xi} \boldsymbol{K}_{\eta} .
$$

L'opération $\boldsymbol{F}$ étant par hypothèse semi-additive, elle est à plus forte raison monotone (lem. 16 $6^{\mathrm{a}}$. Par conséquent, d'après le lem.15 ${ }^{\mathfrak{a}}$, les formules (7) entraînent: $\boldsymbol{F}(\boldsymbol{Y}) \subset \boldsymbol{F}\left(\sum_{\eta<\xi} \boldsymbol{K}_{\eta}\right)$; d'où selon (2) $\boldsymbol{F}(\boldsymbol{Y}) \subset$ $C \boldsymbol{K}_{\xi}$; en vertu de (3) on en obtient:

$$
\boldsymbol{F}(\boldsymbol{Y}) \subset L
$$

Les formules (5) et (8) donnent aussitôt:

$$
X_{\epsilon} L \text {. }
$$

Nous avons ainsi montré que (4) entraîne constamment (9). On a donc l'inclusion: $F^{\prime}(\boldsymbol{L}) \subset \boldsymbol{L}$, d'où en raison de la déf. $16 \quad \boldsymbol{L}_{\epsilon} \mathcal{Q} \mathcal{L}\left(\boldsymbol{F}^{\prime}\right)$ : comme de plus, selon (1) et (3), $K \subset \boldsymbol{L}$, on parvient à la formule:

$$
\boldsymbol{L}_{\in} \boldsymbol{V}(\boldsymbol{K}) \cdot \mathfrak{e}\left(\boldsymbol{F}^{\prime}\right) \text {. }
$$

Or, on peut prouver que $\boldsymbol{L}$ est la plus petite classe de la famille $\boldsymbol{V}(\boldsymbol{K}) \cdot \mathcal{C}\left(\boldsymbol{H}^{\prime}\right)$. Envisageons dans ce but une classe arbitraire $\boldsymbol{X}$ telle que

$$
\boldsymbol{X}_{\epsilon} \boldsymbol{V}(\boldsymbol{K}) \cdot \mathcal{O P}\left(\boldsymbol{F}^{\prime}\right)
$$

Par une induction facile on obtient la formule:

$$
\boldsymbol{K}_{\xi} \subset \boldsymbol{X} \text { pour } \xi<\omega_{\beta} .
$$

En effet, en vertu de (1) et (11) le nombre $\xi=0$ vérifie cette formule. Soit donné ensuite un nombre $\xi, 0<\xi<\omega_{\beta}$, et admettons que tous les nombres $\eta<\xi$ remplissent la formule (12) On a done $\sum_{\eta<\xi} \boldsymbol{K}_{\eta} \subset \boldsymbol{X} ;$ la fonction $\boldsymbol{F}$ étant monotone (comme il a été dit plus haut), on en conclut à l'aide du lem. $15^{\mathrm{a}}$ que $F\left(\sum_{\eta<\xi} K_{\eta}\right) \subset$ $\subset \boldsymbol{F}(\boldsymbol{X})$, d'où en raison de (2) $\boldsymbol{K}_{\xi} \subset \boldsymbol{F}(\boldsymbol{X})$. Comme d'autre part, d'après la déf. 16, la formule (11) donne: $\boldsymbol{F}(\boldsymbol{X}) \subset \boldsymbol{X}$, on en obtient: $\boldsymbol{K}_{\xi} \subset \boldsymbol{X}$, ce qui veut dire que le nombre $\xi$ remplit également la formule en question.

La formule (12), établie tout à l'heure, implique que $\sum_{\xi<\omega_{\beta}} K_{\xi} \subset \boldsymbol{X}$, d'où, selon (3), $\boldsymbol{L} \subset \boldsymbol{X}$. On voit ainsi que la classe $\boldsymbol{L}$ est contenue dans toute classe de la famille $\boldsymbol{V}(\boldsymbol{K}) \cdot \mathscr{C}\left(\boldsymbol{F}^{\prime}\right)$; on a donc

$$
\boldsymbol{V}(\boldsymbol{K}) \cdot \mathfrak{Q} \mathcal{P}\left(\boldsymbol{F}^{\prime}\right) \subset \boldsymbol{V}(\boldsymbol{L}) .
$$

En ce qui concerne la puissance des classes $\boldsymbol{K}_{\xi}$ et $\boldsymbol{L}$, observons en premier lieu les inégalités:

$$
\begin{aligned}
& \overline{\overline{\boldsymbol{K}}} \leqslant(\overline{\overline{\boldsymbol{K}}})^{x_{\beta}} \\
& \aleph_{\beta} \leqslant(\overline{\overline{\boldsymbol{K}}})^{{ }^{*}},
\end{aligned}
$$

qn'on déduit facilement du lem. $5^{\text {c,d }}$ (en tenant compte de l'inégalité: $\overline{\bar{K}} \geqslant 2$ ).

En appliquant une fois encore le principe de l'induction transfinie, nous allons établir la formule:

$$
\overline{\overline{\boldsymbol{K}}} \bar{\xi}(\overline{\overline{\boldsymbol{K}}})^{\boldsymbol{\alpha}_{\beta}} \text { pour } \quad \xi<\omega_{\beta} .
$$

En vertu de (1) et (14),

Soit ensuite $\xi$ un nombre ordinal tel que

$$
0<\xi<\omega_{\beta}
$$

et supposons que

(19) tout nombre $\eta, \eta<\xi$, vérifie la formule (16).

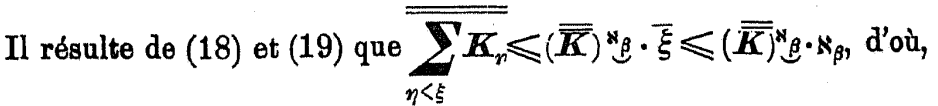


en raison de (15), $\overline{\overline{\sum_{\eta<\xi} \boldsymbol{K}_{\eta}}} \leqslant(\overline{\bar{K}})^{\mathrm{s}_{\beta}}$. En appliquant le lemme $5^{\mathrm{b}}$, on en conclut aussitôt que: $\left(\overline{\overline{\sum_{\eta \zeta \xi} \boldsymbol{K}_{\eta}}}\right)^{\mathrm{N}} \mathrm{B}_{\beta} \leqslant\left((\overline{\overline{\boldsymbol{K}}})^{\mathrm{N}_{\beta}}\right)^{\mathrm{N}_{\beta}}$. Comme on a par hypothèse: $c f(\beta)=\beta$, le lem. $8^{\mathfrak{a}} \quad($ pour $\mathfrak{a}=\overline{\bar{K}}$ et $\gamma=\beta$ ) donne: $\left.\left((\overline{\overline{\boldsymbol{K}}})^{\mathrm{s}_{\beta}}\right)_{\mathcal{B}}^{\mathrm{N}_{\beta}}=\overline{\overline{\boldsymbol{K}}}\right)^{\mathrm{N}_{\beta}}$, et on obtrent finalement la formule:

$$
\left.\overline{\overline{\sum_{\eta<\xi} \boldsymbol{K}_{\eta}}}\right)^{N_{\beta}} \leqslant(\overline{\overline{\boldsymbol{K}}})^{\mathrm{s}_{\beta}} .
$$

D'autre part, conformément à l'hyṕothèse du théorème, on a:

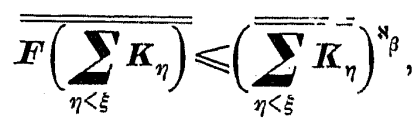

d'où, en raison de $(2)$,

$$
\overline{\boldsymbol{K}}_{\xi} \leqslant\left(\overline{\sum_{\eta<\xi} \boldsymbol{K}_{\eta}}\right)^{k} .
$$

Les inégalités $(20)$ et $(21)$ donnent aussitôt: $\overline{\overline{\boldsymbol{K}}}_{\xi} \leqslant\left(\overline{\overline{\boldsymbol{K}}}^{{ }^{*}}{ }_{\xi} ;\right.$ en d'autres termes

$$
\text { le nombre } \xi \text { vérifie la formule (16). }
$$

Il est ainsi démontré que les conditions (18) et (19) entraînent toujours (22); en tenant compte de (17), on parvient done a la conclusion que tout nombre $\xi<\omega_{\beta}$ vérifie la formule (16).

Dé (3) et (16) on déduit facilemenı: $\overline{\overline{\boldsymbol{L}}} \leqslant \sum_{\xi<\omega_{\beta}} \overline{\overline{\boldsymbol{K}}}_{\xi} \leqslant(\overline{\overline{\boldsymbol{K}}})^{N_{\beta}} \cdot \aleph_{\beta}$, d'où en vertu de (15)

$$
\overline{\overline{\boldsymbol{L}}} \leqslant\left(\overline{\overline{\boldsymbol{K}}}^{{ }^{*}}{ }^{\beta} .\right.
$$

Les formules (10), (13) et (23) prouvent que la classe $\boldsymbol{L}$ satis fait à toutes les conditions du théorème.

Il est à noter que dans l'hypothèse: $c f(\beta)<\beta$ l'inégalité: $\overline{\bar{L}} \leqslant(\overline{\bar{K}})^{*}{ }^{*}$,
, figure dans la thèse du thèorèmo qui figure dans la thèse da thèorème précédent, doit être remplacée par une inégalité plus faible, à savoir: $\overline{\overline{\boldsymbol{L}}} \leqslant(\overline{\overline{\boldsymbol{K}}})^{\boldsymbol{*}_{\beta}}$.
Le but de cet article étant de déterminer les puissances des familles $\mathcal{C}\left(\boldsymbol{F}^{\prime}\right)$ d'après ta puissance de l'ensemble universel 1 dans les cas de différentes opérations particulières $\boldsymbol{F}$, notons d'abord que dans le cas général il n'est possible d'établir que les bornes: inférieure et supérieure de ces puissances, comme le montre le théorème suivant, qui est d'ailleurs d'un caractère tout à fait banal:

Théorème 32. Si $\overline{\bar{I}}=\aleph_{\alpha}$, on a $1 \leqslant \overline{\overline{\mathcal{C f}(F)}} \leqslant 2^{2^{2 \alpha}} ;$ il existe des opérations $\boldsymbol{H}^{\prime}$ telles que $\overline{\overline{\mathcal{C}(\bar{F})}}=1$ et il en existe aussi des opérations $\boldsymbol{F}, p$. ex. $\boldsymbol{F} \stackrel{\circ}{=} \boldsymbol{I}$, telles que $\overline{\overline{\mathcal{Q} \ell(\bar{F})}}=2^{2^{\mathrm{N} \alpha}}$.

Démonstration. En raison du lem. $10^{\mathrm{a}}$ on a pour tout ensemble $A: \overline{\overline{U V(\bar{U})}}=2^{\overline{\overline{U(A)}}}=2^{2^{\overline{\bar{A}}}}$, d'où en particulier $\overline{\bar{U} \bar{U}(1)}=$ $=2^{2^{\mathrm{N} \alpha}}$. En rapprochant cette formule des th. 20 et $23^{\mathrm{a}}$, on obtient

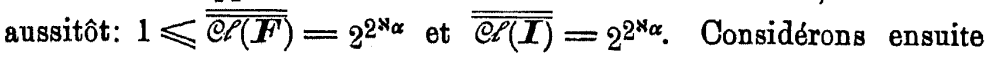
l'opération $\boldsymbol{F}$ définie par la formule: $\boldsymbol{F}(\boldsymbol{X})=\boldsymbol{U}(1)$ pour toute classe $\boldsymbol{X}$. A l'aide de la déf. 16 on vérifie facilement que $\mathcal{C}_{t}(\boldsymbol{F})=$ $=\{U(1)\}$, donc que $\overline{\overline{\mathcal{Q P}(F)}}=1$, ce qui achève la démonstration du théorème.

On a d'habitade affaire anx opérations $\boldsymbol{F}$ qui vérifient la formule: $\boldsymbol{F} \subset \boldsymbol{U} \boldsymbol{\Sigma}$ (c.-à-d. $\boldsymbol{F}(\boldsymbol{X}) \subset \boldsymbol{U}(\boldsymbol{\Sigma}(\boldsymbol{X}))$ pour toute classe $\boldsymbol{X}$ ). Or, en se bornant aux opérations de cette sorte et à leurs opérations doubles, on peut établir pour la puissance de $\mathcal{Q} \ell(\boldsymbol{F})$ nne délimitation plas précise que celle du th. 32 , notamment $2^{\mathrm{N} \alpha} \leqslant \overline{\overline{e_{\ell}(F)}} \leqslant 2^{2^{\mathrm{N} \alpha}}$ (cf. le th. 49 du §5).

Théorème 33. $\overline{\overline{\mathcal{C} \ell\left(\boldsymbol{F}^{*}\right)}}=\overline{\overline{\mathcal{C} \ell\left(\boldsymbol{F}^{\prime}\right)}}$.

Démonstration. La fonction $\boldsymbol{C}(\boldsymbol{X})$ est biunivoque; si en effet, $\boldsymbol{C}(\boldsymbol{X})=\boldsymbol{C}(\boldsymbol{Y})$, on a $\boldsymbol{C C}(\boldsymbol{X})=\boldsymbol{C C}(\boldsymbol{Y})$, donc d'après le lem. $18^{\mathrm{a}}$ et la déf. 13 on obtient: $\boldsymbol{X}=\boldsymbol{Y}$. Il en résulte que les familles $\mathfrak{Q} \ell(\boldsymbol{F})$ et $\overline{\boldsymbol{C}}(\mathfrak{C} \ell(\boldsymbol{F}))$ ont les puissances égales, d'où en raison $d u$ th. $26 \overline{\overline{\mathcal{Q} \ell\left(\boldsymbol{F}^{*}\right)}}=\overline{\overline{\mathscr{C} \ell(\boldsymbol{F})}}$, c. q. f. d.

L'importance du théorème précédent pour les problèmes fondamentaux de ces recherches n'éxige pas d'explications.

Lorsqu'il s'agit de déterminer la puissance de la famille $\mathcal{e} \ell(F)$ dans divers cas particuliers, le lemme suivant rend parfois des services considérables : 
Lemme 34. a) Si $F^{2} \stackrel{\circ}{=}$ et $I \check{C}^{\circ} \boldsymbol{F}$ et si en outre la classe d'ensembles $\boldsymbol{K}$ vérifie la formule: $\boldsymbol{U}(\boldsymbol{K}) \subset \underset{\boldsymbol{X}}{\mathbb{K}}[\boldsymbol{K} \cdot \boldsymbol{F}(\boldsymbol{X}) \subset \boldsymbol{X}]$, on a $\overline{\overline{\mathcal{Q} \ell(\boldsymbol{F}}}) \geqslant 2^{\overline{\overline{\boldsymbol{K}}}}$

$\left.{ }^{b}\right)$ si $d$ plus $\overline{\overline{1}}=\aleph_{\alpha}$ et $\overline{\bar{K}}=2^{\mathrm{N} \alpha}$, on a $\overline{\overline{\bar{e} \ell\left(\boldsymbol{F}^{\prime}\right)}}=2^{2^{\mathrm{N} \alpha}}$.

Démonstration a) Considérons deux classes $\boldsymbol{X}$ et $\boldsymbol{Y}$ assujetties aux conditions:

$$
\boldsymbol{X}_{e} \boldsymbol{U}(\boldsymbol{K}), \quad \boldsymbol{Y} \in \boldsymbol{U}(\boldsymbol{K})
$$

$$
\boldsymbol{F}(\boldsymbol{X})=\boldsymbol{F}(\boldsymbol{Y})
$$

Suivant l'hypothèse les formules (1) donnent:

$$
\boldsymbol{K} \cdot \boldsymbol{F}(\boldsymbol{X}) \subset \boldsymbol{X} \text { et } \boldsymbol{K} \cdot \boldsymbol{F}(\boldsymbol{X}) \subset \boldsymbol{Y} .
$$

Comme $I^{\circ} \mathcal{F}$, on a d'après le lèm. $17^{i} X \subset \boldsymbol{F}(\boldsymbol{X})$ et $\boldsymbol{Y} \subset$ $\subset \boldsymbol{F}(\boldsymbol{Y})$, donc en vertu de (1) $\boldsymbol{X} \subset \boldsymbol{K} \cdot \boldsymbol{F}(\boldsymbol{X})$ et $\boldsymbol{Y} \subset \boldsymbol{K} \cdot \boldsymbol{F}(\boldsymbol{Y})$; en tenant compte de (3), on en conclut que

$$
\boldsymbol{K}=\boldsymbol{K} \cdot \boldsymbol{F}(\boldsymbol{X}) \text { et } \boldsymbol{Y}=\boldsymbol{K} \cdot \boldsymbol{F}(\boldsymbol{Y}) .
$$

De (2) et (4) on obtient aussitôt l'égalité:

$$
\boldsymbol{X}=\boldsymbol{Y} \text {. }
$$

Ainsi les formules (1) et (2) entraînent constamment (5). Il en résulte que la fonction $F$ transforme d'une façon biunivoque la famille $\boldsymbol{U}(\boldsymbol{K})$ en $\overline{\boldsymbol{F}} \boldsymbol{U}(\boldsymbol{K})$; ces familles sont done de puissance égale, d'où en raison du lem. $10^{\mathrm{a}}$

$$
\overline{\overline{\overline{\boldsymbol{F}} \boldsymbol{U}(\overline{\boldsymbol{K}})}}=2^{\overline{\overline{\boldsymbol{K}}}}
$$

D'autre part l'inclusion évidente: $\boldsymbol{K} \subset \boldsymbol{U}(1)$ implique que $\boldsymbol{U}(\boldsymbol{K}) \subset$ $\subset U U(1)$ et $\bar{F} U(K) \subset \bar{F} U U(1)$; à l'aide du th. $23^{\mathrm{d}}$ et en vertu de l'hypothèse nous en concluons que

$$
\overline{\boldsymbol{F}} \boldsymbol{U}(\boldsymbol{K}) \subset \mathcal{e}\left(\boldsymbol{F}^{\prime}\right) .
$$

Les formules (6) et (7) donnent aussitôt:

$$
\overline{\bar{Q}\left(\overline{\boldsymbol{F}^{\prime}}\right)} \geqslant 2 \overline{\overline{\boldsymbol{K}}} \text {, e. q. f. d. }
$$

b) résulte facilement de ${ }^{a}$ ) et du th. 32.

Il serait désirable d'avoir les généralisations de ce lemme, dans lesquelles les conditions qui concernent l'opération $\boldsymbol{F}$ (surtout la condition: $\boldsymbol{F}^{\mathbf{2}}=\mathbf{F}$ ) soient remplacées par des conditions moins restrictives.

Quant aux opérations $F$ qui sont simultanément itératives, adjonctives et monotones, on peut démontrer l'équivalence des formules: $\boldsymbol{U}(\boldsymbol{K}) \subset \underset{\boldsymbol{X}}{\mathbb{E}}[\boldsymbol{K} \cdot \boldsymbol{F}(\boldsymbol{X}) \subset \boldsymbol{X}]$ et $\boldsymbol{U}(\boldsymbol{K}) \cdot \underset{\boldsymbol{X}}{\boldsymbol{E}}[\boldsymbol{F}(\boldsymbol{X})=\boldsymbol{F}(\boldsymbol{K})]=\{\boldsymbol{K}\}$,

dont la première figure dans l'hypothèse da lem. 34; les classes $\boldsymbol{K}$ qui vérifient la deuxième formule pourraient être appelées irréductibles par rapport à l'opération $\boldsymbol{F}$. Il est aisé d'apercevoir que toute sons-classe $\boldsymbol{X}$ d'une classe $K$ remplissant l'hypothèse da lem. $34^{\mathrm{a}}$ remplit également cette hypothèse; cette remarque est de nature à faciliter la détermination de la puissance de la famille des classes de cette sorte.

Le lem. 34 fournit une méthode de déterminer la puissance de la famille $\mathcal{C}(\boldsymbol{F})$ dans le cas où l'opération $\boldsymbol{F}$ est itérative et adjonctive. Cette méthode, dont j'aurai à faire l'usage plusieurs fois, consiste dans la construction d'une classe $\boldsymbol{K}$ remplissant les hypothèses $\mathrm{du}$ lem. $34^{\mathrm{a}}$ et ayant en outre la plus haute puissance possible, c.-à-d. $2^{\mathrm{N}_{\alpha}}$ (où $\overline{\overline{1}}=\aleph_{\alpha}$ ); il résulte alors du lem, $34^{\text {í }}$ que la famille $\mathcal{O}(\boldsymbol{F})$ est aussi de la plus haute puissance possible, c.-à.d. $2^{2 \mathrm{N \alpha}}$.

Il est manifeste que celte méthode n'est pas toujours appliquable: elle est en défaut dans tous les cas où chaque classe $\boldsymbol{K}$ assujettie aux conditions du lemme, et que nous savons construire, est de la puissance $<2^{\star \alpha}$. Bien qu'il soit encore possible dans ce cas d'obtenir à l'aide du lem. $34^{\mathrm{a}}$ une délimitation intérieure de la puissance de $\mathscr{C}\left(\mathbb{F}^{\prime}\right)$, il est plus facile d'ordinaire parvenir à ce résultat par une autre voie, Ainsi, dans le cas général, la classe vide pent être la senle classe $\boldsymbol{K}$ qui vérifie la formule: $\boldsymbol{U}(\boldsymbol{K}) \subset \frac{E}{\boldsymbol{E}}[\boldsymbol{K} \cdot \boldsymbol{F}(\boldsymbol{X}) \subset \boldsymbol{X}]$; le lem. $34^{\text {a }}$ ne donne alors qu'une délimitation banale: $\overline{\overline{\mathcal{Q}(F)}} \geqslant 1$. Dans l'hypothèse que $\boldsymbol{F}^{\circ} \subset^{\circ} \boldsymbol{U} \boldsymbol{\Sigma}$, cette formule est remplie, comme on voit facilement, par toute classe d'ensembles disjoints. Par conséquent, il y a parmi les classes considérées des classes de puissance $\aleph_{\alpha}$, p. ex. $\boldsymbol{K}=\bar{J}(1)=\underset{\langle x\rangle}{E}[x \in 1]$, d'où en raison du lem. $34^{\mathrm{a}} \overline{\overline{\hat{\mathscr{C}\left(F^{\prime}\right)}}} \geqslant 2^{\mathrm{ka}}$; or, la même délimitation sera obtenue dans le th, 49 du $\$ 5$ par une voie plas simple.

Fundamenta Mathematicae. T. XVI. 
La méthode qui vient d'être décrite sera employée tout de suite pour déterminer la puissance de la famille $\mathcal{O}(\boldsymbol{C})$.

Lemme 35. Si $\overline{\overline{1}}=\aleph_{\alpha}$, il existe une classe d'ensembles $\boldsymbol{K}$ vérifiant les formules: $\boldsymbol{U}(\boldsymbol{K}) \subset \underset{\boldsymbol{X}}{E}[\boldsymbol{K} \cdot \boldsymbol{C}(\boldsymbol{X}) \subset \boldsymbol{X}]$ et $\overline{\overline{\boldsymbol{K}}}=2^{\mathrm{s} \alpha}$.

Démonstration. Considérons un élément arbitraire $a$ de 1 et posons :

$$
\boldsymbol{K}=\boldsymbol{U}(\boldsymbol{1}-\{a\})
$$

Il résulte de (1) que pour tout ensemble $Y$ la formule: $Y_{\in} \boldsymbol{K}$ entraîne: $a \in 1-Y$, done $1-Y \bar{\epsilon} \boldsymbol{K}$. Comme d'après la déf. 15 $\boldsymbol{C}(\boldsymbol{X})=\underset{1-Y}{E}\left[Y_{\epsilon} \boldsymbol{X}\right]$, on en conclut que pour toute classe $\boldsymbol{X}$ contenue dans $\boldsymbol{K}$ on a $\boldsymbol{K} \cdot \boldsymbol{C}(\boldsymbol{X})=0$ et a fortiori $\boldsymbol{K} \cdot \boldsymbol{C}(\boldsymbol{X}) \subset \boldsymbol{X}$. Par conséquent

$$
\boldsymbol{U}(\boldsymbol{K}) \subset \underset{\boldsymbol{X}}{E}[\boldsymbol{K} \cdot \boldsymbol{C}(\boldsymbol{X}) \subset \boldsymbol{X}]
$$

On a ensuite $\overline{\overline{1-\{a\}}}=\aleph_{\alpha}-1=\aleph_{\alpha}$; la formule (1) donne done en vertu du lem. $10^{\mathrm{a}}$ :

$$
\overline{\overline{\boldsymbol{K}}}=2^{\mathrm{\alpha} \alpha} \text {. }
$$

En raison de (2) et (3), $K$ est la classe cherchée.

Théorème fondamental I. Si $\overline{\overline{1}}=\aleph_{\alpha}$, on $a \overline{\overline{\mathcal{Q}(\bar{C})}}=2^{2^{\mathrm{N \alpha}}}$.

Démonstration. Soit $\boldsymbol{K}$ une classe arbitraire remplissant la thèse du lemme précédent. Conformément aux déf. $9^{\mathrm{a}}$ et 13 -on a: $\boldsymbol{K} \cdot[\boldsymbol{I}+\boldsymbol{C}](\boldsymbol{X})=\boldsymbol{K} \cdot(\boldsymbol{X}+\boldsymbol{C}(\boldsymbol{X})) \subset \boldsymbol{X}+\boldsymbol{K} \cdot \boldsymbol{C}(\boldsymbol{X}) \subset \boldsymbol{X}$ pour $X \subset K$; par conséquent, on peut transformer les formules du lem. 35
de la façon suivante:

$$
U(\boldsymbol{K}) \subset \underset{\boldsymbol{X}}{E}[\boldsymbol{K} \cdot[\boldsymbol{I}+\boldsymbol{C}](\boldsymbol{X}) \subset \boldsymbol{X}] \text { et } \overline{\bar{K}}=\mathbf{2}^{\mathrm{Na}} .
$$

L'opération $I+C$ étant évidemment adjonctive $(I \check{C} I \stackrel{+}{ }+)$ et de plus itérative (comme le prouve le lem. 18 $)$, on peut appliquer le lem. $34^{\text {b }}$, en y posant: $\boldsymbol{F} \stackrel{+}{=}+\boldsymbol{C}$; en vertu de(1) on en obtient:

$$
\overline{\overline{\mathscr{Q}(I \dot{+}+\boldsymbol{C})}}=2^{2^{\mathrm{N} \alpha}} \text {. }
$$

Comme, en raison du th. $23^{\mathrm{b}}$, $\mathfrak{e}(\boldsymbol{I} \dot{+})=\mathfrak{e} \ell(\boldsymbol{C})$, la formule (2) donne:

$$
\overline{\overline{\mathcal{Q}}(\bar{C})}=2^{2 \alpha \alpha}, \text { c. q.f.d. }
$$

Ce sont exclusivement les ensembles infinis qui nous intéressent ici, bien que plasieurs modes de raisonnement, de même que certains résultats, se prêtent après des modifications convenables à des extensions aux-ensembles finis. Il est à remarquer en particulier que dans le th. $\mathbf{3 2}$ et le lem. 34 le nombre $\aleph_{\alpha}$ peut être remplacé par un nombre cardinal $\mathfrak{a}$ tout à fait arbitraire. Quant au th. fond. I, on peut l'étendre aux nombres finis sous la forme suivante:

$$
\text { Si } \overline{\bar{I}}=\mathfrak{a}>0, \quad \text { on } a \quad 2^{2^{\mathfrak{a}-1}} \leqslant \overline{\overline{\mathcal{Q} l(C)}} \leqslant 2^{2^{\mathfrak{a}}} .
$$

\section{§ 4. Opération $T$. Classes d'ensembles héréditaires.}

A partir de ce $\S$ nos recherches ne concernent que des opérations spéciales.

L'opération qui sera mise au premier plan et que je vais désigner par $\boldsymbol{T}$ consiste à ajouter à une classe d'ensembles $\boldsymbol{K}$ donnée tous les sous-ensembles des éléments de cette classe. Les classes d'ensembles closes par rapport à cette opération peuvent être appelées dans le language ordinaire classes héréditaires.

$$
\text { Definition 17. } T(K)=\sum_{X \in K} U(X) \text {. }
$$

Notons les propriétés suivantes de l'opération $\boldsymbol{T}$ :

Théorème 36. a) $T \in \mathfrak{Z}$, donc $T \in \mathfrak{A}_{\beta}$ et $T \in \mathfrak{M}$;

b) $T^{2}=T$ et $I \dot{C} T$

-) $\boldsymbol{T}(0)=0$;

d) $\boldsymbol{T}(\{A\})=\boldsymbol{U}(A)$, en particulier $\boldsymbol{T}(\{0\})=\{0\}$

-) si $\boldsymbol{K} \neq 0$, on a $0 \in \boldsymbol{T}(\boldsymbol{K})$;

1) si $1 \in \boldsymbol{K}$, on a $\boldsymbol{T}(\boldsymbol{K})=\boldsymbol{U}(1)$;

s) $E[x \in \Sigma(\boldsymbol{K})] \subset T(\boldsymbol{K})$

h) $s i \quad \Sigma(\boldsymbol{K}) \epsilon \boldsymbol{K}$, on a $\boldsymbol{T}(\boldsymbol{K})=\boldsymbol{U} \boldsymbol{\Sigma}(\boldsymbol{K})$. 


\section{Démonstration est évidente.}

En dehors de l'opération $\boldsymbol{T}$, c'est l'opération $\boldsymbol{T}^{*}$, double de $\boldsymbol{T}$, qui va nous occuper ici; comme nous allons le voir, elle consiste à ajouter à une classe $\boldsymbol{K}$ donnée tous les sur-ensembles des éléments de cette classe.

Théorème 37. $T^{*}(\boldsymbol{K})=\sum_{X \in K} V(X)$.

Démonstration résulte des déf. 15 et 17 .

Certaines propriétés de l'opération $T^{*}$ se laissent déduire aisément à l'aide du lem. 19 des propriétés correspondantes de l'opération $\boldsymbol{T}$ qui viennent d'être formulées dans le th. $36^{\mathrm{a}, \mathrm{b}, \mathrm{c}}$. Je vais donner ici quelques autres propriétés de $\boldsymbol{T}^{*}$.

Theorème 38. a) Si $\quad K \neq 0$, on a $1 \in T^{*}(\boldsymbol{K})$;

b) si $0 \in \boldsymbol{K}$, on a $\boldsymbol{T}^{*}(\boldsymbol{K})=\boldsymbol{V}(0)=\boldsymbol{U}(1)$;

-) quelles que soient les classes $\boldsymbol{K}$ et $\boldsymbol{L}$, les trois formules: $\boldsymbol{T}(\boldsymbol{K}) \cdot \boldsymbol{L}=0, \quad \boldsymbol{T}(\boldsymbol{K}) \cdot T^{*}(\boldsymbol{L})=0 \quad$ et $\boldsymbol{K} \cdot T^{*}(\boldsymbol{L})=0 \quad$ sont équivalentes;

d) $\boldsymbol{T} T^{*}(0)=0=T^{*} T(0) ; \quad T T^{*}(K)=U(1)=T^{*} \boldsymbol{T}(\boldsymbol{K})$ pour $\boldsymbol{K} \neq 0$;

ө) $T T^{*} \stackrel{\circ}{=} T^{*} T \stackrel{\circ}{=} T T^{*}$;

1) pour que $\boldsymbol{F} \subset \boldsymbol{C} \boldsymbol{T} \boldsymbol{T}^{*}$, il faut et il suffit que $\boldsymbol{F}(0)=0$.

Démonstration. Les couditions a) et ${ }^{b}$ ) s'obtiennent aussitôt du th. 37 .

c) Supposons que $\boldsymbol{T}(\boldsymbol{K}) \cdot T^{*}(\boldsymbol{L}) \neq 0$; soit p. ex. $Z_{\in} \boldsymbol{T}(\boldsymbol{K}) \cdot \boldsymbol{T}^{*}(\boldsymbol{L})$. D'après la déf. 17 et le th. 37 , il existe donc des ensembles $X$ et $Y$ tels que $X \in \boldsymbol{K}, Y_{\epsilon} L$ et $Y \subset Z \subset X$, d'où $Y \subset Z$. En appliquant une fois encore la déf. 17 et le th. 37 , on en obtient: $X_{\in} \boldsymbol{K} \cdot \boldsymbol{T}^{*}(\boldsymbol{L})$ et $Y_{\boldsymbol{\epsilon}} \boldsymbol{T}(\boldsymbol{K}) \cdot \boldsymbol{L}$; par conséquent $\boldsymbol{K} \cdot \boldsymbol{T}^{*}(\boldsymbol{L}) \neq 0$ et $\boldsymbol{T}(\boldsymbol{K}) \cdot \boldsymbol{L} \neq 0$.

On conclut de ce raisonnement par contraposition que chacune des formules: $\boldsymbol{T}(\boldsymbol{K}) \cdot \boldsymbol{L}=0$ et $\boldsymbol{K} \cdot \boldsymbol{T}^{*}(\boldsymbol{L})=0$ entraîne: $\boldsymbol{T}(\boldsymbol{K}) \cdot \boldsymbol{T}^{*}(\boldsymbol{L})=0$. En tenant compte des inclusions: $\boldsymbol{K} \subset \boldsymbol{T}(\boldsymbol{K})$ et $\boldsymbol{L} \subset \boldsymbol{T}^{*}(\boldsymbol{L})$ (que l'on déduit du th. $36^{\mathrm{b}}$ à l'aide des lom. $19^{\mathrm{y}}$ et $17^{f}$ ), on se convaint sans peine que les implications inverses se présentent aussi. Les trois formules sont donc équivalentes, c. q. f. d. d) En vertu du th. $36^{\mathrm{c}}$ et du lem. $19^{\mathrm{k}}$ on obtient: $\boldsymbol{T}(0)=0=$ $=\boldsymbol{T}^{*}(0)$, done $\boldsymbol{T} \boldsymbol{T}^{*}(0)=0=\boldsymbol{T}^{*} \boldsymbol{T}(0)$. Si par contre $\boldsymbol{K} \neq 0$, on a $1 \in \boldsymbol{T}^{*}(\boldsymbol{K})$ et $0 \in \boldsymbol{T}(\boldsymbol{K})$ (th. $38^{\mathrm{a}}$ et $36^{\circ}$ ), d'où en raison des th. $36^{\mathfrak{f}}$ ét $38^{\mathrm{b}}: \boldsymbol{T} \boldsymbol{T}^{*}(\boldsymbol{K})=\boldsymbol{U}(1)=\boldsymbol{T}^{*} \boldsymbol{T}(\boldsymbol{K})$. Les formules ${ }^{d}$ ) sont ainsi établies.

${ }^{\text {e) }}$ et ${ }^{\text {f) }}$ résultent de d) et du lem. $18^{\mathrm{d}}$.

Deux opérations arbitraires $\boldsymbol{F}$ et $\boldsymbol{G}$ qui remplissent la condition suivante: les formules $\boldsymbol{F}(\boldsymbol{X}) \cdot \boldsymbol{Y}=0$ et $\boldsymbol{X} \cdot \boldsymbol{G}(\boldsymbol{Y})=0$ sont equivalentes pour toutes deux classes $\boldsymbol{X}$ et $\boldsymbol{Y}$, peuvent être appelées conjuguées; conformément au th. $38^{\circ}, \boldsymbol{T}$ et $\boldsymbol{T}^{*}$ sont done des opérations conjuguées. J'avais démontré. à ce propos le théorème génèral suivant ${ }^{1}$ ):

L'additivité totale d'une opération $\boldsymbol{F}$ donnée est une condition à la fois necéssaire et suffisante pour que $\boldsymbol{F}$ admette une opération conjuguée.

J'ai omis, bien entendu, dans les th. 36 et 38 plasieurs propriétés élémentaires des opérations $\boldsymbol{T}$ et $\boldsymbol{T}^{*}$ dont je ne ferai pas usage dans

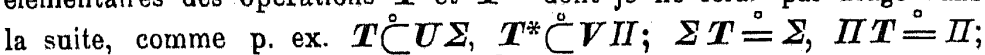
$\overline{\overline{\boldsymbol{\Sigma}}(\overline{\boldsymbol{K}})} \leqslant \overline{\overline{\boldsymbol{T}(\overline{\boldsymbol{K}})}} \leqslant 2^{\overline{\overline{\Sigma(\bar{K})}}} ; T^{*} J \stackrel{\circ}{=} ; \mathfrak{B}(\{\boldsymbol{C}, \boldsymbol{T}\})=\left\{\boldsymbol{I}, \boldsymbol{C}, \boldsymbol{T}, T^{*}\right.$, $\left.\boldsymbol{C} \boldsymbol{T}, \boldsymbol{C} \boldsymbol{T}^{*}, \boldsymbol{T} \boldsymbol{T}^{*}\right\}$ et $\mathfrak{B}(\{\boldsymbol{C}, \boldsymbol{T}\})=\mathcal{S}_{\mathfrak{B}}(\{\boldsymbol{C}, \boldsymbol{T}\})$. De plus, certaines formales que l'on trouve dans les théorèmes precédents penvent être mises dans une antre forme, moins intaitive, mais plus concise, p. ex.: $\boldsymbol{T} \stackrel{\boldsymbol{\Sigma}}{\mathrm{\Sigma}} \overline{\boldsymbol{U}}$ (déf. 17), $\boldsymbol{T} J \stackrel{\circ}{=}\left(\right.$ th. $\left.36^{\text {d }}\right), \bar{J} \Sigma \stackrel{\circ}{\complement}^{T}$ (th. $36^{\text {g }}$ ) et $\boldsymbol{T}^{*} \stackrel{\circ}{=} \overline{\boldsymbol{V}}$ (th. 37); cf. ici les remarques de la p. 209.

Il est ramarquable que les denx opérations $\boldsymbol{T}$ et $\boldsymbol{T}^{*}$ satisfunt à tous les postalats que M. K u ra tow вki a admis dans sa Thèse pour l'opération de fermeture ${ }^{2}$ ); comme une autre opération de cette nature on peut citer $\boldsymbol{I}+\overline{\boldsymbol{J}} \boldsymbol{\Sigma}$.

Ce sont des classes eloses par rapport aux opérations $\boldsymbol{T}$ et $\boldsymbol{T}^{*}$ qui seront étudiées dans la suite de ce §.

Théord̀me 39. $\mathscr{C}\left(\boldsymbol{T}^{*}\right)=\underset{\boldsymbol{U}(1)-\boldsymbol{X}^{*}}{E}\left[\boldsymbol{X}_{\epsilon} \mathscr{Q} \mathcal{P}(\boldsymbol{T})\right]$.

Démoustration. Leth. $38^{\text {c, }}$, si l'on y pose: $\boldsymbol{L}=\boldsymbol{U}(1)-\boldsymbol{T}(\boldsymbol{K})$, donne: $\boldsymbol{T}(\boldsymbol{K}) \cdot \boldsymbol{T}^{*}(\boldsymbol{U}(1)-\boldsymbol{T}(\boldsymbol{K}))=0$; la fonction $\boldsymbol{T}^{*}$ admettant comme valeurs des classes d'ensembles, on a en outre évidemment $\boldsymbol{T}^{*}(\boldsymbol{U}(1)-\boldsymbol{T}(\boldsymbol{K})) \subset \boldsymbol{U}(1)$. Par conséquent,

$$
T^{*}(\boldsymbol{U}(1)-\boldsymbol{T}(\boldsymbol{K})) \subset \boldsymbol{U}(1)-T(\boldsymbol{K}) .
$$

1) Cf. ma communication: Sur quelques propriétés caractéristiques des images dionsertion de Varsovie, Ann. de la Soc. Pol, de Math, VI, p. 127 -128.

2) Sur l'operation $\bar{A}$ d'Analysis Situs, Fund. Math. III, p. 182-199. 
En vertu du th. $36^{\text {b }}$ et du lem. $19^{1}$ on obtient:

$$
I \dot{C} T^{*}
$$

"d'où suivant le lem. $17^{p}$ (pour $\boldsymbol{F}^{\circ} \stackrel{\circ}{=} \boldsymbol{T}^{*}$ )

$$
\boldsymbol{U}(1)-\boldsymbol{T}(\boldsymbol{K}) \subset \boldsymbol{T}^{*}(\boldsymbol{U}(1)-\boldsymbol{T}(\boldsymbol{K})) ;
$$

en rapprochant cette inclusion de (1), on conclut aussitôt que

(3) $\boldsymbol{T}^{*}(\boldsymbol{U}(1)-\boldsymbol{T}(\boldsymbol{K}))=\boldsymbol{U}(1)-\boldsymbol{T}(\boldsymbol{K})$ pour toute classe $\boldsymbol{K}$.

D'une façon tout à fait analogue on établit la formule:

(4) $\boldsymbol{T}\left(\boldsymbol{U}(1)-\boldsymbol{T}^{*}(\boldsymbol{L})\right)=\boldsymbol{U}(\mathcal{1})-\boldsymbol{T}^{*}(\boldsymbol{L})$ pour toute classe $\boldsymbol{L}$.

Or, soit $\boldsymbol{X}$ une classe arbitraire de $\mathcal{Q}(\boldsymbol{T})$ et $\boldsymbol{Y}=\boldsymbol{U}(1)-\boldsymbol{X}$. En raison des th. $23^{\mathrm{c}}$ (pour $\boldsymbol{F}=\boldsymbol{T}$ ) et $36^{\mathrm{b}}$ on a $\boldsymbol{T}(\boldsymbol{X})=\boldsymbol{X}$; on posant dans la formule (3): $\boldsymbol{K}=\boldsymbol{X}$, on en obtient: $\boldsymbol{T}^{*}(\boldsymbol{Y})=\boldsymbol{Y}$ donc, d'après la déf. 16, $\boldsymbol{Y}_{\epsilon} \mathcal{C}\left(\boldsymbol{T}^{*}\right)$. A l'aide des formules (2) et (4) on prouve de même qu'à toute classe $\boldsymbol{Y}$ de $\mathcal{C}\left(\boldsymbol{T}^{*}\right)$ vient correspondre une classe $\boldsymbol{X}$ (à savoir $\boldsymbol{X}=\boldsymbol{U}(1)-\boldsymbol{Y}$ ) telle que $\boldsymbol{Y}=$ $=U(1)-\boldsymbol{X}$ et $\boldsymbol{X}_{\epsilon} \varrho \ell(\boldsymbol{T})$.

Nous avons ainsi établi l'équivalence des deux conditions suivantes: (a) $\boldsymbol{Y}_{\epsilon} \mathcal{C} \ell\left(\boldsymbol{T}^{*}\right)$ et (b) il existe une classe $\boldsymbol{X}$ vérifiant les formules: $\boldsymbol{Y}=\boldsymbol{V}(1)-\boldsymbol{X}$ et $\boldsymbol{X} \in \mathcal{Q} \mathcal{C}(\boldsymbol{T})$. Il en résulte immediatement l'identité cherchée:

$$
\mathcal{Q}\left(\boldsymbol{T}^{*}\right)=\underset{\boldsymbol{U}(1)-\boldsymbol{X}}{E}\left[\boldsymbol{X}_{\epsilon} \mathcal{Q}(\boldsymbol{T})\right]
$$

$\mathscr{H}$ étant une famille de classes, posons, par analogie complète avec la déf. 15: $\mathfrak{C}(\mathscr{H})=\underset{\boldsymbol{U}(\mathfrak{I})-\boldsymbol{X}}{E}\left[\boldsymbol{X}_{\epsilon} \mathscr{H}\right]$; le th. 39 prend alors la forme suivante: $\mathscr{C}\left(\boldsymbol{T}^{*}\right)=\mathcal{C}(\mathfrak{C}(\boldsymbol{T}))$. Il est instructif de rapprocher cette identité de celle qui s'obtient comme cas particulier du th. 26: $\mathcal{Q}\left(T^{*}\right)=$ $=\bar{C}(\mathcal{Q}(T))$; malgré la ressemblance exlérieure de ces deux formules, l'ane exprime une propriété spécifique de l'operation $\boldsymbol{T}$, pendant que l'autre résulte des propriétés les plus générales de la notion d'opération
donble.

Pour examiner la puissance des familles $\mathfrak{C}(\boldsymbol{T})$ et $\mathcal{C}\left(\boldsymbol{T}^{*}\right)$, il faut rappeler au préalable le lemme suivant, dû à $M . K$ a a te $\mathbf{r}^{1}$ ), mais formulé ici dans les termes un peu différents:

1) Cf. C. Kuratowski, Sur la puissance des nnombres de dimension“ au sens de M. Fréchet, Fund. Math. VIII, p. 205, note $\$$ ).
Lemme 40, Si $\overline{\overline{1}}=\aleph_{\alpha}$, il existe une classe d'ensembles $\boldsymbol{K}$ vérifiant les formules: $\boldsymbol{U}(\boldsymbol{K}) \subset_{\boldsymbol{X}}[\boldsymbol{K} \cdot \boldsymbol{T}(\boldsymbol{X}) \subset \boldsymbol{X}], \quad \boldsymbol{U}(\boldsymbol{K}) \subset_{\boldsymbol{X}}\left[\boldsymbol{K} \cdot \boldsymbol{T}^{*}(\boldsymbol{X}) \subset \boldsymbol{X}\right]$ et $\overline{\overline{\boldsymbol{K}}}=2^{\mathrm{s} \alpha}$.

Démonstration de ce lemme étant connue ${ }^{1}$ ), je me borne à en ésquisser la marche générale.

La formule: $\aleph_{\alpha}=2 \cdot \aleph_{\alpha}$ implique l'existence d'un ensemble $A$ tel que $\overline{\bar{A}}=\overline{\overline{1-A}}=\aleph_{\alpha}$. On en conclut qu'il existe une fonction $f$ qui transforme de façon biunivoque l'ensemble $A$ en son complémentaire $1-A: f(A)=1-A$. Posons $F(X)=X+f(A-X)$ pour $X \subset A$ et $\boldsymbol{K}=\bar{F} \boldsymbol{U}(A)$. Il est aisé à démontrer que les formules $Y_{\epsilon} \boldsymbol{K}, Z_{\varepsilon} \boldsymbol{K}$ et $Z \subset Y$ entraînent constamment: $Y=Z$, donc que $\boldsymbol{K} \cdot \boldsymbol{U}(Y)=\{Y\}$ et $\boldsymbol{K} \cdot \boldsymbol{V}(Z)=\{Z\}$ pour tous ensembles $Y$ et $Z$ de la classe $\boldsymbol{K}$. On en obtient à l'aide de la déf. 17 et du th. 37: $\boldsymbol{K} \cdot \boldsymbol{T}(\boldsymbol{X})=\boldsymbol{K} \cdot \sum_{Y \in X} \boldsymbol{U}(Y)=\sum_{Y_{\in \in} \boldsymbol{X}} \boldsymbol{K} \cdot \boldsymbol{U}(Y)=\sum_{Y_{\in} \boldsymbol{X}}\{Y\}=\boldsymbol{X}$ et d'une manière semblable: $\boldsymbol{K} \cdot \boldsymbol{T}^{*}(\boldsymbol{X})=\boldsymbol{X}$ pour toute sous-classe $\boldsymbol{X}$ de $\boldsymbol{K}$. Par conséquent, la classe $\boldsymbol{K}$ vérifie les formules: $\boldsymbol{U}(\boldsymbol{K}) \subset$ $\subset \underset{\boldsymbol{X}}{\boldsymbol{E}}[\boldsymbol{K} \cdot \boldsymbol{T}(\boldsymbol{X}) \subset \boldsymbol{X}]$ et $\boldsymbol{U}(\boldsymbol{K}) \subset \underset{\boldsymbol{X}}{E}\left[\boldsymbol{K} \cdot \boldsymbol{T}^{*}(\boldsymbol{X}) \subset \boldsymbol{X}\right]$

D'autre part, on voit facilement que la fonction $F$ est biunivoque, donc que la classe $U(A)$ est de puissance égale à celle de son image $\bar{F} \boldsymbol{U}(A)=\boldsymbol{K}$; en vertu du lem. $10^{\mathrm{a}}$ on en conclut que $\overline{\overline{\boldsymbol{K}}}=2^{\overline{\bar{A}}}=2^{\mathrm{N} \alpha}$. Ainsi $\boldsymbol{K}$ est, la classe cherchée.

Comme conséquence facile da lemme précédent on obtient le

Thèorème fondamental II. St $1=\aleph_{\alpha}$, on a

$$
\overline{\overline{\mathcal{Q}(\boldsymbol{T})}}=\overline{\overline{\mathcal{Q}\left(\boldsymbol{T}^{*}\right)}}=2^{2 * \alpha} \text {. }
$$

Démonstration. En raison du lem. 19, l'opération $\boldsymbol{T}$ étant itérative et adjunctive (th. $36^{\text {b }}$ ), l'opération double $\boldsymbol{T}^{*}$ l'est également. On peut donc appliquer à deux reprises le lem. $34^{\mathrm{b}}$, en $\mathrm{y}$ posant: $\boldsymbol{F}^{\prime} \stackrel{\circ}{=} \boldsymbol{T}$ et puis $\boldsymbol{F}^{\circ} \stackrel{\circ}{=} \boldsymbol{T}^{*}$; en tenant compte du lem. 40, on parvient aussitôt à la formule cherchée: $\overline{\overline{\mathscr{C}(T)}}=\overline{\overline{\mathcal{Q} P\left(T^{*}\right)}}=2^{2^{\text {*a }}}$.

Ce théorème a été acquis par l'auteur de cet ouvrage en collaboration avec M. Lind enbaum.

1) Cf. la note précédente. 
En analysant la démonstration du théoréme précédent, on obtient un théorème un peu plus général, qui est appliquable au cas d'ensembles finis.

Si $\overline{\bar{I}}=\mathfrak{a}_{2}$ on $a 2^{2^{E\left(\frac{\mathfrak{a}}{2}\right)}} \leqslant \overline{\overline{\mathcal{C C}(T)}}=\overline{\left.\overline{\mathcal{C}\left(T^{*}\right.}\right)} \leqslant 2^{2^{\mathfrak{a}}}$ (le symboile $E\left(\frac{\mathfrak{a}}{\mathfrak{2}}\right)$ désignant le plus grand des nombres $\mathfrak{r}$ qui vérifient la formule: $2 \cdot \mathfrak{x} \leqslant \mathfrak{a})$.

Il est à noter que la formule: $\overline{\overline{\mathfrak{C} l(T)}}=\overline{\overline{\mathscr{C}\left(T^{*}\right)}}$ établie dans le th. fond. II s'obtient facilement du th. 39; d'ailleurs elle ne présente qu'un cas particulier da th. 33 .

Quant aux classes closes par rapport aux deux opérations $\boldsymbol{T}$ et $\boldsymbol{I}^{*}$ à la fois, on a le suivant

Théorème 41. a) $\mathfrak{C l}(C \stackrel{+}{T})=\mathfrak{C l}\left(C+T^{*}\right)=\mathfrak{C} C\left(T+T^{*}\right)=$ $=\{0, U(1)\}$

b) si $\boldsymbol{H}(0)=0$, on a $\mathfrak{C}(\boldsymbol{C}+\boldsymbol{T}+\boldsymbol{H})=\mathfrak{C}\left(\boldsymbol{C}+\boldsymbol{T}^{*}+\boldsymbol{H}\right)=$ $=\mathfrak{e}\left(\boldsymbol{T}+\boldsymbol{T}^{*}+\boldsymbol{H}\right)=\{0, \boldsymbol{U}(1)\}$.

Démonstration. a) En vertu du th. $36^{\mathrm{a}, \mathrm{b}}$, on peut faire appel au th. $28^{\text {a }}$ (pour $\boldsymbol{F} \stackrel{\circ}{=}$ ); en tenant compte du th. $38^{\circ}$, on en conclut que $\mathfrak{C}(\boldsymbol{C}+\boldsymbol{T})=\mathfrak{C}\left(\boldsymbol{C}+\boldsymbol{T}^{*}\right)=\mathfrak{C} \mathcal{L}\left(\boldsymbol{C} \boldsymbol{T} \boldsymbol{T}^{*}\right)=\mathcal{Q} \mathscr{C}\left(\boldsymbol{T} \boldsymbol{T}^{*}\right)$. De

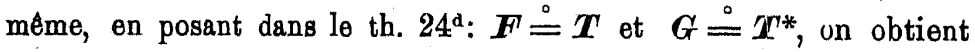
en raison du th. $36^{\mathrm{a}, \mathrm{b}}$ et du lem. $19^{\mathrm{j}}: \mathcal{e}\left(\boldsymbol{T}+\boldsymbol{T}^{*}\right)=\mathcal{C}\left(\boldsymbol{T} \boldsymbol{T}^{*}\right)$. Or, si l'on rapproche du th. $38^{\mathrm{d}}$ la déf. 16, on se convaint sans peine qu'il n'y a que deux classes d'ensembles closes par rapport à l'opération $\boldsymbol{T} \boldsymbol{T}^{*}$, à savoir 0 et $\boldsymbol{U}(1)$ : $\mathcal{C}\left(\boldsymbol{T} \boldsymbol{T}^{*}\right)=\{0, \boldsymbol{U}(1)\}$. On parvient ainsi à la formule a) cherchée:

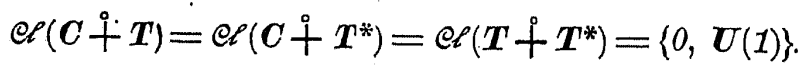

b) Conformément à la déf. 16, la formule: $\boldsymbol{F}(0)=0$ donne: $0 \in \mathcal{C} \mathcal{P}(\boldsymbol{H})$; comme en outre, d'après le th. $20, \boldsymbol{U}(1) \in \mathcal{Q} P(\boldsymbol{H})$, on obtient: $\{0, \boldsymbol{U}(1)\} \subset$ $C \mathcal{Q}(\boldsymbol{H})$. D'autre part, le th. $21^{\mathrm{b}}$ (pour $\boldsymbol{F}=\boldsymbol{C}+\boldsymbol{T}$ et $\left.\boldsymbol{G} \stackrel{\circ}{=} \boldsymbol{H}\right)$ entraîne: $\mathcal{C}(\boldsymbol{C}+\boldsymbol{T}+\boldsymbol{H})=\mathcal{Q}(\boldsymbol{C}+\boldsymbol{T}) \cdot \mathcal{C}(\boldsymbol{H})$, d'où en vertu de la formule a): $\mathscr{C}(\boldsymbol{C}+\boldsymbol{T}+\boldsymbol{H})=\{0, \boldsymbol{U}(1)\} \cdot \mathscr{C}(\boldsymbol{H})$. On en conclut aussitôt que $\mathcal{C}(\boldsymbol{C}+\boldsymbol{T}+\boldsymbol{H})=\{0, \boldsymbol{U}(1)\}$; les autres parties de la formule $\left.{ }^{b}\right)$ s'obtiennent d'une façon analogue.

Comme la formule $\boldsymbol{H}(0)=0$ est remplie par toutes les opérations dont nous avons affaire ici, le théorème qui vient d'être établi nous permet de négliger dans les recherches ultérieures les opérations de la forme $\boldsymbol{C}+\boldsymbol{T}+\boldsymbol{H}, \boldsymbol{C}+\boldsymbol{T}^{*}+\boldsymbol{H}$ et $\boldsymbol{T}+\boldsymbol{T}^{*}+\boldsymbol{H}$.

Théorème fondamental 1II. $\overline{\overline{\mathscr{C} C(C+T)}}=\overline{\overline{\mathscr{C}\left(C+T^{*}\right)}}=$ $=\overline{\overline{\mathcal{Q P}\left(\boldsymbol{T}+\boldsymbol{T}^{*}\right)}}=2$.

C'est une conséquence immédiate du th. $41^{\mathrm{a}}$.

Nous laissons an lecteur d'examiner les propriétés de l'opération $\boldsymbol{T}^{\times}$ (c.-à-d. de la deuxième opération double de $\boldsymbol{T}$; ef. la fin du § 2 ) et en parliculier d'élablir les théorèmes suivants:

A. Si $\overline{\overline{1}}=\aleph_{\alpha}$, ori $a \overline{\overline{\mathcal{C P}\left(T^{x}\right)}}=2^{2^{* \alpha}}$ et $\overline{\overline{\mathcal{Q P}\left(T+T^{\circ}\right)}}=2^{\alpha_{\alpha}}$.

B. $\mathcal{C} \ell\left(C+\boldsymbol{T}^{\times}\right)=\{0, \boldsymbol{U}(1)\}$ et $\overline{\overline{\mathcal{Q}\left(\boldsymbol{C}+\boldsymbol{T}^{\times}\right.}}=2$.

\section{§ 5. Opération $\boldsymbol{S}$. Classes d'ensembles additives.}

Je passe à l'opération $\boldsymbol{S}$ qui consiste à former tous les ensembles-sommes de sous-classes quelconques d'une classe d'ensembles $\boldsymbol{K}$ donnée; les classes closes par rapport à cette opération seront appelées parfois classes additives.

Définition 18. $\boldsymbol{S}(\boldsymbol{K})=\bar{\Sigma}(\boldsymbol{U}(\boldsymbol{K})-\{0\})$.

L'opération $\boldsymbol{S}^{\prime}(\boldsymbol{K})=\bar{\Sigma}(\boldsymbol{U}(\boldsymbol{K}))$, qui parait plus naturelle, est moins commode à cause de la propriété: $0 \in \boldsymbol{S}^{\prime}(\boldsymbol{K})$, qui se présente pour tout $\boldsymbol{K}$, donc mếme pour $\boldsymbol{K}=0$. En conséquence les th. $42^{\mathrm{c}, \mathrm{d}}$ et $43^{\mathrm{d}, \theta}$ seraient en défant.

Certaines propriétés élémentaires de l'opération $\boldsymbol{S}$ sont données dans le suivant

Théorème 42. a) $S \in \mathfrak{M}$;

v) $\boldsymbol{S}^{2} \stackrel{\circ}{=} \boldsymbol{S}$ et $\boldsymbol{I} \complement^{\circ} \boldsymbol{S}$;

c) $\boldsymbol{S}(0)=0$;

d) $\boldsymbol{\Sigma} \boldsymbol{S}(\boldsymbol{K})=\boldsymbol{\Sigma}(\boldsymbol{K})$ et $\Pi \boldsymbol{S}(\boldsymbol{K})=\Pi(\boldsymbol{K})$;

e) si $\boldsymbol{K} \neq 0$, on $\boldsymbol{a} \boldsymbol{\Sigma}(\boldsymbol{K}) \in \boldsymbol{S}(\boldsymbol{K})$;

') si $\boldsymbol{K} \neq 0$ et $\underset{\langle x\rangle}{E}[x \in \boldsymbol{\Sigma}(\boldsymbol{K})] \subset \boldsymbol{K}$, on a $\boldsymbol{S}(\boldsymbol{K})=\boldsymbol{U} \boldsymbol{\Sigma}(\boldsymbol{K})$;

g) $\dot{S}(\boldsymbol{K}) \leqslant 2^{\overline{\boldsymbol{K}}}$.

Démonstration est tout à fait élémentaire. En particulier, la formule: $\boldsymbol{S}^{2} \stackrel{\circ}{=} \boldsymbol{S}$ de ${ }^{b}$ ) se déduit facilement de la loi associative 
générale d'addition des ensembles à l'aide de l'axiome du choix. Si l'on veut cependant éviter ici l'usage de cet axiome, il suffit de remarquer qu'il est possible de définir d'une façon effective une fonction $\boldsymbol{F}$ faisant correspondre à tout ensemble $X$ de $\boldsymbol{S}(\boldsymbol{K})$ une classe d'ensembles $\boldsymbol{F}(X)$ telle que $\boldsymbol{F}(X) \subset \boldsymbol{K}, \boldsymbol{F}(X) \neq 0$,et $X=$ $=\boldsymbol{\Sigma} \boldsymbol{F}(X)$; on pose notammènt: $\boldsymbol{F}(X)=\sum \boldsymbol{Y}$. Cette fonction étant

$$
\boldsymbol{Y} \subset \boldsymbol{K} \text { et } \boldsymbol{\Sigma}(\boldsymbol{Y})=X
$$

en outre biunivoque, son existence entraîne selon le lem. $10^{\text {a }}$ la formule $\mathrm{g}$ ).

Je vais établir à présent quelques relations qui existent entre l'opération $\boldsymbol{S}$ et les opérations $\boldsymbol{T}$ et $\boldsymbol{T}^{*}$ examinées dans le $\S$ précédent.

Théorème 43. a) $T S(0)=0=S T(0) ; \quad$ si $\quad K \neq 0$ on a $\boldsymbol{T} \boldsymbol{S}(\boldsymbol{K})=\boldsymbol{U} \Sigma(\boldsymbol{K})=\boldsymbol{S} \boldsymbol{T}(\boldsymbol{K})$;

b) $\boldsymbol{T} S \stackrel{\circ}{=} \boldsymbol{T}$

c) pour que $\boldsymbol{F}^{\circ} \complement^{\boldsymbol{T}} \boldsymbol{S}$, il faut et il suffit que $\boldsymbol{F}^{\circ} \complement^{\circ} \boldsymbol{V} \boldsymbol{\Sigma}$ et que $\boldsymbol{F}(0)=0$;

d) $S \subset^{\circ} T^{*}$

e) $\boldsymbol{S}(\boldsymbol{K}+\boldsymbol{L}) \subset \boldsymbol{T}^{*}(\boldsymbol{K})+\boldsymbol{S}(\boldsymbol{L})$ pour toutes deux classes $\boldsymbol{K}$ et $\boldsymbol{L}\left(\boldsymbol{S}[\boldsymbol{F}+\boldsymbol{G}] \complement^{\circ} \boldsymbol{T}^{*} \boldsymbol{F}+\boldsymbol{S} \boldsymbol{G}\right.$ pour toutes deux opérations $\boldsymbol{F}$ et $\left.\boldsymbol{G}\right)$.

Dém onstration. ${ }^{\text {a }}$ Les th. $36^{\circ}$ et $42^{\circ}$ donnent aussitôt: $\boldsymbol{T S}(0)=$ $=0=S T(0)$. Si par contre $\boldsymbol{K} \neq 0$, on a en vertu des th. $42^{\circ}$ et $36^{\mathrm{g}}: \boldsymbol{\Sigma}(\boldsymbol{K}) \in \boldsymbol{S}(\boldsymbol{K})$ et $E[x \in \boldsymbol{\Sigma}(\boldsymbol{K})] \subset \boldsymbol{T}(\boldsymbol{K})$, d'où d'après les th. $36^{\mathrm{h}}$ et $42^{\text {f: }} \boldsymbol{T} \boldsymbol{S}(\boldsymbol{K})=\boldsymbol{U} \boldsymbol{\Sigma}^{(\boldsymbol{K})}=\boldsymbol{S} \boldsymbol{T}(\boldsymbol{K})$. Les formules a) sont donc
établies.

$\left.{ }^{b}\right)$ et $\left.{ }^{c}\right)$ s'obtiennent tout de suite de ${ }^{a}$ ). Les formules d) et ${ }^{\text {e) }}$ résultent facilement de la déf. 18 et du th. 37 .

Comme il a été dit (p. 209), l'opération $I$ constitue dans un certain sens la borne inférieure de la plupart des operations qui nous occupent dans cet ouvrage; or, l'opération $\boldsymbol{T} \boldsymbol{S}$ envisagée dans le théorème précédent en constitue daus le même sens la borne supérieure. Les opérations $F$ qui vérifient l'inclusion: $F^{\circ} T S$ (ou, si l'on veut, l'inclusion un peu plus générale: $\boldsymbol{F}^{\circ} \subset \boldsymbol{C} \Sigma$ ) peuvent étre appelées intrinsèques.
Il mérite d'être noté qu'il y a des opérations intrinsèques $\boldsymbol{F}$, comme p. ex. $\boldsymbol{F}^{\circ} \stackrel{\boldsymbol{T}}{ }$, dont les opérations doubles $\boldsymbol{F}^{*}$ ne le sont pas. On connaît cependant un nombre des opérations qui sont intrinsèques avec leurs doubles et que l'on pourrait appeler pour cette raison intrinseques au sens strict; telles sont p. ex. l'opération $S$ et les opérations qui seront définies dans le $\S 6$. On voit facilement que les opérations $\boldsymbol{F}$ de cette sorte sont caracterisées par les formules: $\boldsymbol{F} \subset \boldsymbol{C} \boldsymbol{C} S$ et $\boldsymbol{F} \subset \boldsymbol{T}^{*} \boldsymbol{S}^{*}$

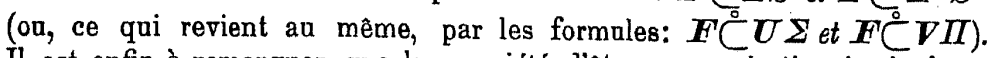
Il est enfin à remarquer que la propriété d'être une opération intrinsèque subsiste, si l'on passe de $\boldsymbol{F}$ à l'opération $\boldsymbol{F}^{\times}$mentionnée à la fin du $\S 2$.

Le théorème suivant, qui ne présente d'aillears qu'une conséquence immédiate des déf. 15 et 18 et des lois bien connues de De Morgan, caractérise l'opération $\boldsymbol{S}^{*}$, double de $\boldsymbol{S}$ :

Théorème 44. $\boldsymbol{S}^{*}(\boldsymbol{K})=\bar{\Pi}(\boldsymbol{U}(\boldsymbol{K})-\{0\})$.

On voit ainsi que l'opération $\boldsymbol{S}^{*}$ consiste à former tous les produits possibles des ensembles d'une classe $\boldsymbol{K}$ donnée. Vu l'importance de cette opération, on pourrait la désigner par un signe spécial, p. ex. $\boldsymbol{P}$; les classes d'ensembles closes par rapport à $\boldsymbol{S}^{*}$ peuvent être appelées classes multiplicatives.

Nous ferons appel dans la suite aux diverses propriétés de l'opération $S^{*}$ qu'on déduit des th. $42^{\mathrm{a}-\mathrm{c}}$ et $43^{\mathrm{b} \text {,de }}$ par l'application du lem. 19. Considérons en outre les propriétés suivantes qui résultent immédiatement du th. 44 (et de la déf. 17):

Théorème 45. a) $\Sigma S^{*}(\boldsymbol{K})=\boldsymbol{\Sigma}(\boldsymbol{K})$ et $\Pi \boldsymbol{S}^{*}(\boldsymbol{K})=\Pi(\boldsymbol{K})$;

$\left.{ }^{b}\right)$ si $\boldsymbol{K} \neq 0$, on a $\Pi(\boldsymbol{K}) \in \boldsymbol{S}^{*}(\boldsymbol{K})$; en particulier, si $\Pi(\boldsymbol{K})=0$, on $\left.a \quad 0 \in \mathcal{S}^{*}(\boldsymbol{K})^{1}\right)$;

c) $\boldsymbol{S}^{*}(\boldsymbol{K}+\boldsymbol{L}) \subset \boldsymbol{T}(\boldsymbol{K})+\boldsymbol{S}^{*}(\boldsymbol{L})$ pour toutes deux classes $\boldsymbol{K}$ et $\boldsymbol{L}$ $\left(\boldsymbol{B}^{*}[\boldsymbol{F}+\boldsymbol{G}] \stackrel{\circ}{\complement} \boldsymbol{T} \boldsymbol{F}+\boldsymbol{S}^{*} \boldsymbol{G}\right.$ pour toutes deux opérations $\boldsymbol{F}$ et $\left.\boldsymbol{G}\right)$.

Dans l'étude approfondie des opérations $\boldsymbol{S}$ et $\boldsymbol{S}^{*}$ (et surtout dans l'étude des classes d'ensembles qui sont à la fois additives et multiplicatives) une opération auxiliaire $\boldsymbol{M}$ rend des services considérables. Cette opération consiste à former des ensembles d'une

1) J'admets ici, comme d'habitude, $\Pi(0)=1$; la formule $\Pi(\boldsymbol{K})=0$ implique done que $\boldsymbol{K} \neq 0$. 
classe $\boldsymbol{K}$ donnée certains autres ensembles dits molécules on atọmes de cette classe. En généralisant notamment une notion introduite par M. Fréchet ${ }^{1}$ ), nous appelons atome de la classe $\boldsymbol{K}$ relatif à l'élément a de $\Sigma(\boldsymbol{K})$, en symboles $A_{\boldsymbol{K}}(a)$, la partie commune (le produit) de tous les ensembles de la classe $\boldsymbol{K}$ qui contiennent l'élément $a$; la classe $\boldsymbol{M}(\boldsymbol{K})$ est formée par tous ces atomes $A_{\boldsymbol{K}}(a)$ (où $a \in \boldsymbol{\Sigma}(\boldsymbol{K})$ ) et de plứs par l'ensemble 0 dans le cas où cet ensemble coïncide avec $\Pi(\boldsymbol{K})$.

$$
\begin{aligned}
& \text { Définition 19. a) } A_{\boldsymbol{K}}(a)=\prod_{a \in X_{\epsilon} \boldsymbol{K}} X \\
& \text { v) } \boldsymbol{M}(\boldsymbol{K})=\overline{A_{\boldsymbol{K}}}(\Sigma(\boldsymbol{K}))+\{0\} \cdot\{I I(\boldsymbol{K})\} .
\end{aligned}
$$

Quelques propriétés de l'opération $\boldsymbol{M}$, qui sont d'importance pour nos recherches, sont formulées dans le suivant

$$
\begin{aligned}
& \text { Théorème 46. a) } M \dot{C}^{\circ} S^{*} \text {; } \\
& \text { v) } \boldsymbol{M S} \doteq \boldsymbol{M} \stackrel{\circ}{=} \boldsymbol{M} \boldsymbol{S}^{*} \text {; } \\
& \text { c) } \boldsymbol{S} M \stackrel{\circ}{ }=\boldsymbol{S} \boldsymbol{S}^{*} \text {; } \\
& \text { d) } \overline{\overline{\boldsymbol{M}(\boldsymbol{K})}} \leqslant \overline{\overline{\Sigma(\overline{\boldsymbol{K}})}}+1 \text {. }
\end{aligned}
$$

Démonstration. a) Soit $\boldsymbol{K}$ une classe arbitraire d'ensembles. D'après la déf. 19a, à tout $x \in \Sigma(\boldsymbol{K})$ correspond une classe $\boldsymbol{Y}$ telle que $\boldsymbol{Y} \subset \boldsymbol{K}, \boldsymbol{Y} \neq 0$ et $A_{\boldsymbol{K}}(x)=\boldsymbol{\Pi}(\boldsymbol{Y})$, à savoir: $\boldsymbol{Y}=\boldsymbol{V}(\{x\}) \cdot \boldsymbol{K}$. Il en résulte que $\overline{A_{\boldsymbol{K}}}(\boldsymbol{\Sigma}(\boldsymbol{K}))=\underset{A_{K}(x)}{E}[x \in \Sigma(\boldsymbol{K})] \subset \bar{\Pi}(\boldsymbol{U}(\boldsymbol{K})-\{0\})$, d'où, en raison du th. $44, \overline{A_{\boldsymbol{K}}}(\boldsymbol{\Sigma}(\boldsymbol{K})) \subset \boldsymbol{S}^{*}(\boldsymbol{K})$. Comme, en outre, suivant le th. $45^{\text {b }},\{0\} \cdot\{\Pi(\boldsymbol{K})\} \subset \boldsymbol{S}^{*}(\boldsymbol{K})$, on en obtient conformément à la déf. $19^{\mathrm{b}}: \boldsymbol{M}(\boldsymbol{K}) \subset \mathcal{S}^{*}(\boldsymbol{K})$ pour toute classe $\boldsymbol{K}$. Par conséquent $M \dot{C}^{\circ} S^{*}$, c. q. f. d.

b) $\boldsymbol{K}$ étant une classe quelconque, on obtient du th. $42^{\mathrm{b}}$ à l'aide des lem. $17^{\mathfrak{f}}$ et $19^{1}$ les inclusions: $\boldsymbol{K} \subset \boldsymbol{S}(\boldsymbol{K})$ et $\boldsymbol{K} \subset \boldsymbol{S}^{*}(\boldsymbol{K})$, d'où pour tout $x$ :

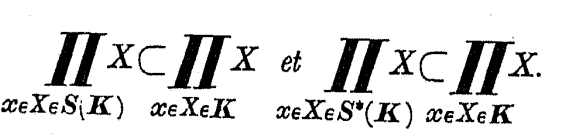
1) Cf. Des familles et fonctions additives d'ensembles abstraits, Fund. Math. V,
p. $210-213$ et $217-218$.
Considérons un ensemble arbitraire $Y$ tel que $x \in Y \in \boldsymbol{S}(\boldsymbol{K})$. Conformément à la déf. $18, Y$ se laisse représenter sous la forme $Y=\boldsymbol{\Sigma}(\boldsymbol{L})$ où $\boldsymbol{L} \subset \boldsymbol{K}$. On a done $x \in \boldsymbol{\Sigma}(\boldsymbol{L})=Y$, ce qui implique l'existence d'un ensemble $Z$ vérifiant les formules: $x \in Z_{\epsilon} L$ ot $Z \subset Y$. Par conséquent $\prod_{x \in X_{\epsilon} \boldsymbol{K}} X \subset \prod_{x \in X_{\epsilon} L} X \subset Z \subset Y$, d'où $\prod_{x \in X_{\epsilon} K} X \subset Y$. Or, cette dernière inclusion étant remplie par tout ensemble $Y$ assujetti à la condition $x \in Y \in \boldsymbol{S}(\boldsymbol{K})$, on en conclut que

\section{$\prod \dot{X} \subset \prod x$ \\ $x \in X_{\epsilon} \boldsymbol{K} \quad x \in X_{\epsilon} S(K)$}

D'une façon aualogue, soit $x \in Y \in S^{*}(\boldsymbol{K})$. D'après le th. 44, il existe une classe $\boldsymbol{L}$ telle que $Y=\Pi(\boldsymbol{L})$ et $\boldsymbol{L} \subset \boldsymbol{K}$. On a done $x \in Z \in \boldsymbol{K}$ pour tout ensemble $Z$ de $L$, d'où $\prod_{x \in X_{\epsilon} \boldsymbol{K}} X \subset Z$. On en obtient ensuite: $\prod_{x \in X_{\epsilon} \boldsymbol{K}} X \subset \prod_{Z_{\epsilon} \boldsymbol{L}} Z=\Pi(\boldsymbol{L})=Y$. Ce raisonnement s'appliquant à tout ensemble $Y$ tel que $x \in Y_{\epsilon} \boldsymbol{S}^{*}(\boldsymbol{K})$, on parvient à la formule:

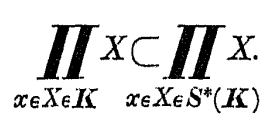

Les inclusions (1)-(3) donnent aussitôt: $\prod x=\prod x=\prod x$, d'où, en raison de la déf. 19a,

$$
\left.x_{\epsilon \in} X_{\epsilon} S_{(K)}\right) \quad x_{\epsilon \in X_{\epsilon} K} \underset{x \in X_{\epsilon} S^{*}}{(K)}
$$

(4) $\quad A_{\boldsymbol{S}(\boldsymbol{K})}(x)=A_{\boldsymbol{K}}(x)=A_{S^{*}(\boldsymbol{K})}(x)$ pour tout élément $x$.

A l'aide de (4) on déduit de la déf. $19^{\mathrm{b}}$ que

(5) $\boldsymbol{M} \boldsymbol{S}(\boldsymbol{K})=\bar{A}_{\boldsymbol{K}}(\boldsymbol{\Sigma} \boldsymbol{S}(\boldsymbol{K}))+\{0\} \cdot\{\boldsymbol{M} \boldsymbol{S}(\boldsymbol{K})\}, \boldsymbol{M}(\boldsymbol{K})=$

$$
\begin{gathered}
=\overline{A_{\boldsymbol{K}}}(\Sigma(\boldsymbol{K}))+\{0\} \cdot\{\Pi(\boldsymbol{K})\} \text { et } \boldsymbol{M} \boldsymbol{S}^{*}(\boldsymbol{K})=\overline{A_{\boldsymbol{K}}}\left(\Sigma \boldsymbol{S}^{*}(\boldsymbol{K})\right)+ \\
+\{0\} \cdot\left\{I I \boldsymbol{S}^{*}(\boldsymbol{K})\right\} .
\end{gathered}
$$

Comme on a en vertu des th. $42^{\mathrm{d}}$ et $45^{\mathrm{a}}: \Sigma \boldsymbol{S}(\boldsymbol{K})=\Sigma(\boldsymbol{K})=\Sigma \boldsymbol{S}^{*}(\boldsymbol{K})$ et $\Pi \boldsymbol{S}(\boldsymbol{K})=\Pi(\boldsymbol{K})=\Pi \boldsymbol{S}^{*}(\boldsymbol{K})$, les formules (15) entraînent: $\boldsymbol{M} \boldsymbol{S}(\boldsymbol{K})=\boldsymbol{M}(\boldsymbol{K})=\boldsymbol{M} \boldsymbol{S}^{*}(\boldsymbol{K})$ pour toute classe $\boldsymbol{K}$; par conséquent,

$$
\boldsymbol{M} \boldsymbol{S}^{\circ} \stackrel{M}{ } \stackrel{\circ}{=} \boldsymbol{M} \boldsymbol{S}^{*}, \text { c. q. f. d. }
$$


c) Envisageons un ensemble arbitraire $X$ tel que

$$
X \in \boldsymbol{K}-\{0\} .
$$

En vertu de (6) $X \subset \Sigma(\boldsymbol{K})$, done $\overline{A_{\boldsymbol{K}}}(X) \subset \overline{A_{\boldsymbol{K}}}(\boldsymbol{\Sigma}(\boldsymbol{K}))$, d'où suivant la déf. $19^{\mathrm{b}}$

$$
\text { (7) } \quad \bar{A}_{\boldsymbol{K}}(X) \subset \boldsymbol{M}(\boldsymbol{K}) \text {; }
$$

comme de plus, $X \neq 0$, on a:

$$
\overline{A_{\boldsymbol{K}}}(X)_{\boldsymbol{a}} \neq 0 .
$$

En tenant compte de (6), on conclut facilement de la déf. $19^{\mathrm{a}}$ que $A_{K}(x) \subset X$ pour tout $x \in X$, done que

$$
\Sigma\left(\overline{A_{K}}(X)\right) \subset X .
$$

D'autre part la déf. $19^{\mathrm{a}}$ implique également que $x \in A_{K}(x)$, quel que soit $x$, d'où pour tout $x$ de $X$ on a $x \in \Sigma\left(\overline{A_{K}}(X)\right)$; il en résulte que $X \subset \Sigma\left(\overline{A_{K}}(X)\right)$. En rapprochant cette inclusion de (9),
on en obtient:

$$
\left.X=\boldsymbol{\Sigma} \overline{\left(A_{\boldsymbol{K}}\right.}(X)\right) \text {. }
$$

Les formules (7), (8) et (10) montrent que l'ensemble $X$ est de la forme $X=\Sigma(\boldsymbol{L})$ où $\boldsymbol{L} \subset \boldsymbol{M}(\boldsymbol{K})$ et $\boldsymbol{L} \neq 0$; antrement dit, $X_{\epsilon} \bar{\Sigma}(\boldsymbol{U} \boldsymbol{M}(\boldsymbol{K})-\{0\})$, donc conformément à la déf. 18:

$$
X \in \boldsymbol{S} \boldsymbol{M}(\boldsymbol{K}) .
$$

Il est ainsi prouvé que la formule (6) entraîne constamment (11); cette implication peut être évidemment exprimée par la formule:

$$
\boldsymbol{K}-\{0\} \subset \boldsymbol{S} \boldsymbol{M}(\boldsymbol{K})
$$

Passons au cas où $X \in \boldsymbol{K} \cdot\{0\}$. On a alors évidemment $X=$ $=0=\Pi(\boldsymbol{K})$, donc $X \epsilon\{0\} \cdot\{\Pi(\boldsymbol{K})\}$, d'où en raison de la déf. $19^{\mathrm{b}}$ : $X \in M(K)$; comme de plus, en vertu du th. $42^{\text {b }}$ et du lem. 17, $\boldsymbol{M}(\boldsymbol{K}) \subset \boldsymbol{S} \boldsymbol{M}(\boldsymbol{K})$, on en obtient: $X_{\in} \boldsymbol{S} \boldsymbol{M}(\boldsymbol{K})$. Par conséquent

$$
\boldsymbol{K} \cdot\{0\} \subset \boldsymbol{S} \boldsymbol{M}(\boldsymbol{K}) \text {. }
$$

Les inclusions (12) et (13) donnent aussitôt: $\boldsymbol{K} \subset \boldsymbol{S M}(\boldsymbol{K})$ pour toute classe $K$; conformément aux déf. 13 et $8^{b}$ on a donc

$$
I \subset \mathcal{C} M \text {. }
$$

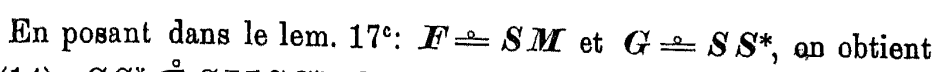
$\operatorname{de}(14): S S^{*} \subset^{0} \boldsymbol{S M M S} \boldsymbol{S}^{*}$. Or, la formule b) du théorème considéré,

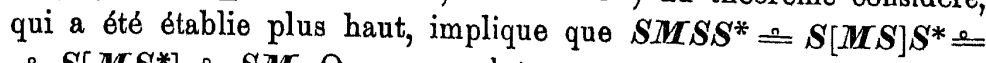

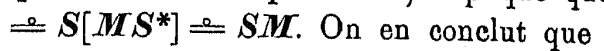

$$
S S^{*} \stackrel{\circ}{\complement} S M \text {. }
$$

D'autre part, en vertu de la formule a), qui a été de même déjà établie, on a $\boldsymbol{M} \subset \boldsymbol{S}^{*}$; cornme en outre $\boldsymbol{S}_{\in} \mathfrak{M}$ (th. $42^{\mathrm{a}}$ ), on en obtient à l'aide de la déf. $12^{\mathrm{a}}$ :

$$
S M \check{C}^{\circ} S S^{*} \text {. }
$$

Les formules (15) et (16) entraînent immédiatement lidentité cherchée :

$$
\boldsymbol{S M} \rightleftharpoons \boldsymbol{S S}^{*} \text {. }
$$

d) Posons dans le lem. 11 a (qui résulte notoirement de l'axiome du choix): $f \bumpeq A_{\boldsymbol{K}}$ et $A=\Sigma(\boldsymbol{K})$; on en obtient: $\overline{\overline{\overline{A_{\boldsymbol{K}}(\boldsymbol{\Sigma}(\boldsymbol{K}))}}} \leqslant$ $\leqslant \overline{\overline{\boldsymbol{\Sigma}(\boldsymbol{K})}}, \quad$ d'uù d'après la dét. $19^{\mathrm{v}} \overline{\overline{\boldsymbol{M}(\overline{\boldsymbol{K}})}} \leqslant \overline{\overline{\overline{\boldsymbol{A}_{\boldsymbol{K}}}(\boldsymbol{\Sigma}(\boldsymbol{K}))}}+$ $+\overline{\{0\} \cdot \overline{\{\Pi(\overline{\boldsymbol{K}})\}}} \leqslant \overline{\overline{\boldsymbol{\Sigma}(\boldsymbol{K})}}+1$, c. q. f. d.

Les propriétés de l'opération $\boldsymbol{M}$ qui viennent d'être établies trouveront une application intéressante dans la démonstration du suivant

Théorème 47. $S S^{*} \doteq S^{*} S$.

Démonstration. Le th. $46^{b}$ donne: $\boldsymbol{S} \boldsymbol{M S}=\boldsymbol{S} \boldsymbol{M}$; à l'aide du th. $46^{\circ}$ on en conclut que

$$
S S^{*} \boldsymbol{S} \doteq S S^{*}
$$

En posant dans le lem. $17^{c}: \boldsymbol{F} \rightleftharpoons \boldsymbol{S}$ et $\boldsymbol{G} \rightleftharpoons \boldsymbol{S}^{*} \boldsymbol{S}$ et en tenant compte du th. $42^{\text {b }}$, nous obtenons ensuite: $\boldsymbol{S}^{*} \boldsymbol{S} \subset \boldsymbol{S} \boldsymbol{S}^{*} \boldsymbol{S}$, d'où selon (1)

$$
\boldsymbol{S}^{*} \boldsymbol{S} \subset \boldsymbol{S} \boldsymbol{S}^{*}
$$

Par l'application du lem. $19^{\mathrm{b}, \mathrm{c}, \mathrm{e}}$ on déduit successivement de (2): $\left[\boldsymbol{S}^{*} \boldsymbol{S}\right]^{*} \mathcal{\complement}\left[\boldsymbol{S} \boldsymbol{S}^{*}\right]^{*}, \boldsymbol{S}^{* *} \boldsymbol{S}^{*} \mathcal{\complement} \boldsymbol{S}^{*} \boldsymbol{S}^{* *}$ et enfin

$$
S S^{*} \stackrel{\circ}{\complement} S^{*} S \text {. }
$$

Les inclusions (2) et (3) donnent aussitôt:

$$
\boldsymbol{S}^{*} \boldsymbol{S}=\boldsymbol{S} \boldsymbol{S}^{*}, \quad \text { c. q. f. d. }
$$


Le théorème précédent se laisse d'ailleurs démontrer par une autre voie, plus naturelle peut.être, mais pas plus simple: au lieu de l'opération $\boldsymbol{M}$, on applique notamment les lois distributives d'addition et de maltiplication des ensembles dans leur forme la plas générale. Toutefois on se hearte à certaines difficultés, si l'on tâche d'éviter dans cette nouvelle démonstration l'asage de l'axiome du choix.

Je vais mentionner ici quelques propriétés des opérations $\boldsymbol{S}, \boldsymbol{S}^{*}$ et $\boldsymbol{M}$ qui ont été omises dans les th. $42-47$. On peut démontrer que $l a$ formule: $\boldsymbol{F} \Sigma \bumpeq \Sigma \overline{\boldsymbol{F}} \boldsymbol{S}$ (c.-̀̀-d. $\boldsymbol{F} \boldsymbol{\Sigma}(\mathscr{O} \mathcal{C})=\boldsymbol{F}(\boldsymbol{X})$ pour toute fa$\boldsymbol{X}_{\in} \widehat{S}(\mathscr{H})$

mille de classes $\mathscr{X}$ ) est une condition à la fois nécessaire et suffisante pour que l'on ait: $\boldsymbol{F} \in \mathfrak{M}$ et $\boldsymbol{F}(0)=0$; on a donc en particulier: $S \Sigma$ e $\Sigma \bar{S} S$. Le th. 29 du $\$ 3$ se laisse formuler de la façon suivante: si $\boldsymbol{F}^{\prime} \in \mathfrak{M}$, on a $\boldsymbol{S}^{*}(\mathcal{C} \mathcal{C}(\boldsymbol{F})) \subset \mathcal{Q} \mathcal{P}(\boldsymbol{F})$. On a ensuite: $\boldsymbol{S} J \bumpeq J$ e

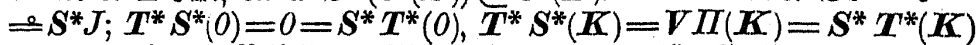
pour $\boldsymbol{K} \neq 0 ; \mathfrak{B}(\{\boldsymbol{C} \boldsymbol{T}, \bar{S}\})=\left\{\boldsymbol{I}, \boldsymbol{C}, \boldsymbol{T}, \boldsymbol{T}^{*}, \boldsymbol{S}, \boldsymbol{S}^{*}, \boldsymbol{C} \boldsymbol{T}, \boldsymbol{C} \boldsymbol{T}^{*}\right.$, $\left.C S, C S^{*}, T T^{*}, T S, T^{*} S^{*}, S S^{*}, C T S, C T^{*} S^{*}, C S S^{*}\right\} . L^{\prime o-}$ pération $\boldsymbol{M}$ vérifie en outre les formules: $\boldsymbol{M}^{\mathrm{a}} \supseteq \boldsymbol{M} ; \bar{J}(1) \cdot \boldsymbol{K} \subset \boldsymbol{M}(\boldsymbol{K})$; $\boldsymbol{M}(0)=0 ; \boldsymbol{\Sigma} \boldsymbol{M} \bumpeq \boldsymbol{\Sigma} ; \boldsymbol{M} \boldsymbol{T}(0)=0, \boldsymbol{M} \boldsymbol{T}(\boldsymbol{K})=\bar{J} \boldsymbol{\Sigma}(\boldsymbol{K})+\{0\}$ pour $\boldsymbol{K} \neq 0 ; \boldsymbol{M} \boldsymbol{T} \in \boldsymbol{X},[\boldsymbol{M} \boldsymbol{T}]^{2} \bumpeq \boldsymbol{M} \boldsymbol{T} ; \boldsymbol{S}^{*} \boldsymbol{M} \cong \boldsymbol{M}$. Il est à noter que $\boldsymbol{M}(\boldsymbol{K})$ n'est pas dans le cas général une classe d'ensembles disjoints.

Passons aux problèmes qui concernent les classes d'ensembles additives et multiplicatives.

Théorème fondamental IV. Si $\overline{\bar{I}}=\kappa_{\alpha}$, on a $\overline{\overline{\mathcal{U}(\bar{S})}}=\overline{\overline{\mathcal{C}\left(S^{*}\right)}}=$ $=2^{2 N \alpha}$.

Démonstration. Le th. $21^{\mathrm{a}}$ donne en vertu du th. $43^{\mathrm{d}}$ :

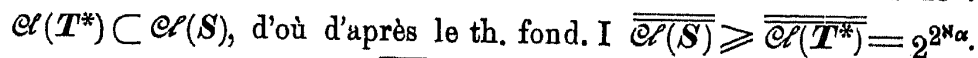
L'inégalitè inverse, c.-à-d. $\overline{\overline{\mathcal{O P}(\boldsymbol{S})}} \leqslant 2^{2^{\mathrm{N} \alpha}}$, résulte immédiatement $\mathrm{du}$ th. 32. Comme on a de plus, en raison du th. $33, \overline{\overline{\mathcal{C} \ell(S)}}=\overline{\overline{\mathcal{C}\left(\boldsymbol{S}^{*}\right)}}$, on obtient finalement: $\overline{\overline{\mathcal{Q} P(S)}}=\overline{\overline{\mathcal{Q} P\left(S^{*}\right)}}=2^{2^{\mathrm{N} \alpha}}$, c. q. f. $\mathrm{d}$.

Ce théorème m'a été communiqué par M. Lindenbaum.

Théorème 48. a) $\mathfrak{Q}(\boldsymbol{T}+\boldsymbol{S})=\mathfrak{Q}(\boldsymbol{T} \boldsymbol{S})=\{0\}+\overline{\boldsymbol{U}} \boldsymbol{U}(1)$;

b) $\mathscr{C}\left(\boldsymbol{T}^{*}+\boldsymbol{S}^{*}\right)=\mathfrak{Q} \mathcal{C}\left(\boldsymbol{T}^{*} \boldsymbol{S}^{*}\right)=\{0\}+\overline{\boldsymbol{V}} \boldsymbol{V}(0)=\{0\}+\overline{\boldsymbol{V}} \boldsymbol{U}(1)$.

Démonstration. a) En tenant compte des th. $36^{a, b}$ ot $42^{b}$, on obtient da th. $24^{\mathrm{d}}$ :

(1)

$$
\mathcal{Q}(\boldsymbol{T}+\boldsymbol{S})=\mathcal{Q}(\boldsymbol{T} \boldsymbol{S})
$$

Envisageons une classe arbitraire $\boldsymbol{Y}$ de $\mathcal{C}(\boldsymbol{T S})$, différente de 0 . Conformément à la déf. $16 \boldsymbol{T S}(\boldsymbol{Y}) \subset \boldsymbol{Y}$, d'où en raison du th. $43^{\mathrm{a}}$ $\boldsymbol{U} \boldsymbol{\Sigma}(\boldsymbol{Y}) \subset \boldsymbol{Y}$; en outre, on a évidemment $\boldsymbol{Y} \subset \boldsymbol{U} \boldsymbol{\Sigma}(\boldsymbol{Y})$ (pour toute classe $\boldsymbol{Y})$. Par conséquent $\boldsymbol{Y}=\boldsymbol{U} \boldsymbol{\Sigma}(\boldsymbol{Y})$; la classe $\boldsymbol{Y}$ est done de la forme $\boldsymbol{Y}=\boldsymbol{U}(\boldsymbol{X})$, où $X=\boldsymbol{\Sigma}(\boldsymbol{Y})$ appartient à la classe $\boldsymbol{U}(1)$ (comme ensemble d'individus).

Il résulte de ce raisonnement que $\mathcal{e}(\boldsymbol{T} \boldsymbol{S}) \subseteq\{0\}+\underset{U(X)}{E}\left[\boldsymbol{X}_{\epsilon} \boldsymbol{U}(\boldsymbol{I})\right]$ Or, d'après la déf. 6 (pour $f=U$ et $A=U(1)$ ), cette inclusion peut être mise sous la forme:

$$
\mathcal{Q} P(\boldsymbol{T S}) \subset\{0\}+\bar{U} \boldsymbol{U}(1)
$$

D'autre part, soit $\boldsymbol{Y} \in\{0\}+\overline{\boldsymbol{U}} \boldsymbol{U}(1)$. Si $\boldsymbol{Y} \in\{0\}$, on a $\boldsymbol{T S}(\boldsymbol{Y})=\boldsymbol{Y}$,

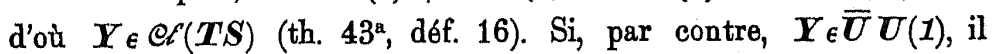
existe un ensemble $X$ tel que $\boldsymbol{Y}=\boldsymbol{U}(X)$ (et $X_{\epsilon} \boldsymbol{U}(1)$ ). On en conclut aussitôt que $\boldsymbol{\Sigma}(\boldsymbol{Y})=\boldsymbol{\Sigma} \boldsymbol{U}(X)=X$, d'où $\boldsymbol{Y}=\boldsymbol{U} \boldsymbol{\Sigma}(\boldsymbol{Y})$ et, suivant le th. $43^{\mathrm{a}}, \boldsymbol{Y}=\boldsymbol{T} \boldsymbol{S}(\boldsymbol{Y})$; en appliquant une fois encore la déf. 16 , on en obtient done de nouveau la formule: $\boldsymbol{Y}_{\epsilon} \mathcal{Q P}(\boldsymbol{T S})$.

Il est aînsi prouvé que

$$
\{0\}+\bar{U} U(1) \subset \mathcal{Q}(\boldsymbol{T S}) \text {. }
$$

Les formules (1)-(3) entraînent tout de suite:

$$
\mathscr{C}(\boldsymbol{T}+\boldsymbol{S})=\mathcal{Q} \mathscr{P}(\boldsymbol{T S})=\{0\}+\overline{\boldsymbol{U}} \boldsymbol{U}(\boldsymbol{1}), \text { c. q. f. d. }
$$

b) A l'aide du th: 26 on obtient facilement de $\left.{ }^{a}\right): \mathfrak{e t}\left([\boldsymbol{T}+\boldsymbol{S}]^{*}\right)=$ $=\mathcal{e} \mathcal{L}\left([\boldsymbol{T} \boldsymbol{S}]^{*}\right)=\overline{\boldsymbol{C}}(\{0\}+\overline{\boldsymbol{U}} \boldsymbol{U}(1))$, d'où en vertu du lem. $19^{\mathrm{d}, \mathrm{e}}$ $\mathcal{Q}\left(\boldsymbol{T}^{*}+\boldsymbol{S}^{*}\right)=\mathcal{C} \mathcal{P}\left(\boldsymbol{T}^{*} \boldsymbol{S}^{*}\right)=\overline{\boldsymbol{C}}(\{0\})+\overline{\boldsymbol{C}} \overline{\boldsymbol{U}} \boldsymbol{U}(1)$. Or, le lem. 18d donne: $\bar{C}(\{0\})=\{0\}$, et à l'aide des déf. 5,6 et 14 on se convaint sans peine que $\bar{C} \bar{U} U(1)=\underset{C U(X)}{E}\left[X_{\epsilon} U(1)\right]=\underset{V(1-X)}{E}\left[X_{\epsilon} U(1)\right]=$ $=E\left[Y_{\epsilon} U(1)[=\overline{\boldsymbol{V}} \boldsymbol{U}(1)\right.$. On parvient ainsi à la formule cherchée: $V(Y)$

$\mathcal{C}_{\iota}\left(T^{*}+S^{*}\right)=\mathfrak{Q} \ell\left(T^{*} S^{*}\right)=\{0\}+\bar{\nabla} U(1)=\{0\}+\bar{\nabla} \boldsymbol{V}(0)$.

Théorème fondamental V. Si $\overline{\overline{1}}=\aleph_{\alpha}$, on a $\overline{\overline{\mathcal{C}(T+S)}}=$ $=\overline{\overline{\mathcal{C}\left(T^{*}+S^{*}\right)}}=2^{* \alpha}$.

Démonstration. $U$ est évidemment une fonction biunivoque: si $U(X)=U(Y)$, on a $\Sigma U(X)=\Sigma U(Y)$, d'où $X=Y$. Par Fondamenta Mathomatica. T. XVI. 
conséquent, la classe $U(1)$ est de la même puissance que son image

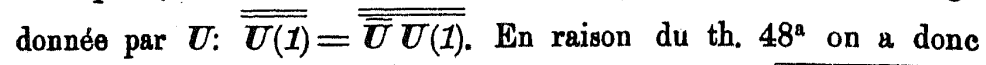
$\mathscr{C}(\boldsymbol{T}+\boldsymbol{S})=\overline{\overline{\boldsymbol{U}(1)}}+\overline{\overline{\{0}}$, d'où en vertu du lem. $10^{\mathrm{a}} \overline{\overline{\mathcal{Q P}(\boldsymbol{T}+\overline{\boldsymbol{S}})}}=$ $=2^{\alpha_{\alpha}}+1=2^{\alpha \alpha}$. Comme en outre, d'après le th. 33 et le lem. $19^{\alpha}$,

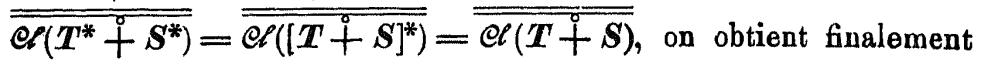
la formule cherchée: $\overline{\overline{\mathscr{Q}(\boldsymbol{T}+\boldsymbol{S})}}=\overline{\overline{\mathscr{Q} \mathcal{C}\left(\boldsymbol{T}^{*}+\boldsymbol{S}^{*}\right)}}=2^{\boldsymbol{N}_{\alpha}}$.

A l'aide du théorème précédent, il est aisé de déterminer la borne inférieure des puissances des familles $\mathcal{O}\left(\boldsymbol{F}^{\prime}\right)$ qui correspondent aux opérations $F$ intrinsèques et à leurs opérations doubles (tandis que la borne supérieure de ces puissancés reste la même que dans le cas général):

Théorème 49. Si $\overline{\bar{I}}=\aleph_{\alpha}$, chacune des formules: $\boldsymbol{H}^{\prime} \mathcal{C}^{\circ} \boldsymbol{T}+\boldsymbol{S}$, $\boldsymbol{F}^{\circ} \boldsymbol{C} \boldsymbol{T S}, \boldsymbol{F}^{\circ} \dot{C} T^{*}+\boldsymbol{S}^{*}$ et $\boldsymbol{F}^{\circ} \boldsymbol{C}^{*} \boldsymbol{S}^{*}$ entruîne: $2^{* \alpha} \leqslant \overline{\overline{\mathcal{O} P(\boldsymbol{H}})} \leqslant 2^{2 \alpha_{\alpha}}$.

Démonstration. D'après th. $21^{\text {a }}$ la formule: $\boldsymbol{F}^{\circ} \boldsymbol{C}+\boldsymbol{T}$ donne: $\mathscr{C}(\boldsymbol{T}+\boldsymbol{S}) \subset \mathcal{C}\left(\boldsymbol{F}^{\prime}\right)$, d'où, en raison du th. fond.V, $2^{\alpha_{\alpha}} \leqslant \overline{\overline{\mathcal{C} \ell\left(\boldsymbol{F}^{\prime}\right)}}$. En rapprochant cette inégalité da th. 32 , on en obtient aussitôt la formule cherchée: $\left.2^{\alpha_{\alpha}} \leqslant \overline{\overline{\mathcal{C}(\bar{F}}}\right) \leqslant 2^{2^{* \alpha} \alpha}$. En tenant compte du th. 48 , on raisonne dans les autres hypothèses d'une façon complètement analogue.

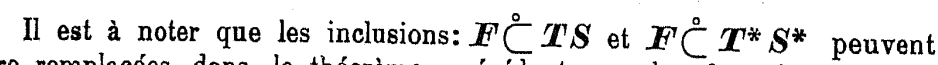
être remplacées dans le théorème précédent par les formules un peu plus générales, à savoir: $F C^{\circ} U \Sigma$ et $\boldsymbol{F}^{\circ} \mathrm{C} \Pi$.

Théorème fondamental VI. $S i \overline{\bar{I}}=s_{\alpha}$, on a $\overline{\overline{\mathscr{C}\left(S+S^{*}\right)}}=2^{\mathrm{N}_{\alpha}}$.

Démonstration. D'après le th. $42^{\mathrm{a}, \mathrm{b}}$ et le lem. $19^{\prime}$, on a $S_{\epsilon} \mathfrak{M}$, $I \complement^{\circ} S$ et $I \subset^{\circ} S^{*}$; en vertu du th. $24^{\mathrm{a}}$ il en résulte que

$$
\mathscr{e}\left(\boldsymbol{S}+\boldsymbol{S}^{*}\right)=\mathscr{Q}\left(\boldsymbol{S} \boldsymbol{S}^{*}\right)
$$

Suivant le lem. 17", les inclusions: $I \complement^{\circ} S$ et $I \complement^{\circ} S^{*}$ donnent ensuite: $\boldsymbol{I} \subset \boldsymbol{S} S^{*}$. On a de plus $S^{2} \supseteq S,\left[S^{*}\right]^{2} \supseteq S^{*}$ et $S S^{*} \rightleftharpoons S^{*} S$ (th. $42^{\mathrm{b}}$, lem. $19^{\mathrm{f}}$ et th. 47 ), d'où en raison du lem. $14^{\mathrm{e}}\left[\boldsymbol{S S ^ { * } ] ^ { 2 }}=S \boldsymbol{S}^{*}\right.$. L'opération $S^{*}$ étant done itérative et adjonctive, on peut appliquer

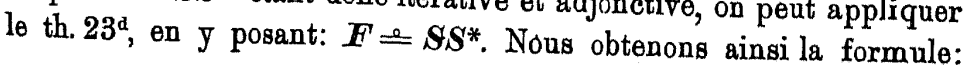

$\mathscr{e}\left(\boldsymbol{S S}^{*}\right)=\overline{\mathbf{S S ^ { * }}} \boldsymbol{U} \boldsymbol{U}(1)$, qui, rapprochée de (1), donne: $\mathcal{C}\left(\boldsymbol{S}+\boldsymbol{S}^{*}\right)=$ $=\overline{S^{*}} \boldsymbol{U U}(1)$; comme en vertu du th. $46^{c} S S^{*}=S M$, il vient:

$$
\mathcal{Q}\left(S+S^{*}\right)=\overline{S M} \boldsymbol{U} \boldsymbol{U}(1) \text {. }
$$

Or, conformément à la propriété connue des images, on a $\overline{f g}(A)=$ $f \bar{g}(A)$ pour toutes deux fonctions $f$ et $g$ et pour tout ensemble $A$. On a donc en particulier $\overline{\boldsymbol{S} M \boldsymbol{M}} \boldsymbol{U} \boldsymbol{U}(1)=\overline{\boldsymbol{S}} \overline{\boldsymbol{M}} \boldsymbol{U} \boldsymbol{U}(1)$. En posant dans le lem. 11a: $f \circ S$ et $A=\overline{\boldsymbol{M}} \boldsymbol{U} \boldsymbol{U}(1)$, on en obtient: $\overline{\overline{\overline{S M T} \boldsymbol{U} \boldsymbol{U}(1)}} \leqslant \overline{\overline{\overline{\boldsymbol{M}} \boldsymbol{U} \boldsymbol{U}(1)}}$, d'où selon (2)

$$
\overline{\overline{\mathscr{Q}\left(S+\boldsymbol{S}^{*}\right.}} \leqslant \overline{\overline{\overline{M U U(1)}} .}
$$

Envisageons une classe arbitraire $\boldsymbol{X}$ de $\boldsymbol{U} \boldsymbol{U}(1)$. En raison du th. $46^{\text {d }}$, on a $\overline{\overline{\boldsymbol{M}(\boldsymbol{X})}} \leqslant \overline{\overline{\boldsymbol{Y}(\boldsymbol{X})}}+1$; comme par hypothèse $\overline{\bar{I}}=\aleph_{\alpha}$ et en outre $\boldsymbol{\Sigma}(\boldsymbol{X}) \subset 1$, on en conclut que $\overline{\overline{\boldsymbol{M}(\overline{\boldsymbol{X}})}} \leqslant \boldsymbol{\aleph}_{\alpha}+1=\aleph_{\alpha}<$ $<\aleph_{\alpha+1}$. De plus, d'après la déf. $19, \boldsymbol{M}(\boldsymbol{X})$ est une classe d'ensembles, donc $\boldsymbol{M}(\boldsymbol{X}) \subset \boldsymbol{U}(1)$; en tenant compte de la déf. $5^{\mathrm{b}}$ (pour $A=\boldsymbol{U}(1)$ ), on en obtient: $\boldsymbol{M}(\boldsymbol{X}) \epsilon \boldsymbol{U}_{a+1} \boldsymbol{U}(1)$.

Ce raisonnement prouve que $\bar{M} U \boldsymbol{U}(1) \subset \boldsymbol{U}_{\alpha+1} \boldsymbol{U}(1)$, d'où

$$
\overline{\overline{\overline{\boldsymbol{M} U U(1)}}} \leqslant \overline{\overline{\boldsymbol{U}_{\alpha+1} \boldsymbol{U}(1)}} .
$$

A l'aide des lem. $10^{\mathrm{a}, \mathrm{c}}$ et $6^{\mathrm{b}}$ on obtient facilement: $\overline{\overline{U_{\mathrm{a}+1} \overline{U(1)}}} \leqslant$ $\leqslant(\overline{\overline{\boldsymbol{U}(1)}})^{\alpha_{\alpha+1}}=\left(2^{\alpha_{\alpha}}\right)^{\alpha_{\alpha+1}}=\left(2^{N_{\alpha}}\right)^{\alpha_{\alpha}}=2^{\mathrm{N}_{\alpha}^{2}}=2^{\mathrm{N}_{\alpha}}$; en le rapprochant de (3) et (4), on en déduit l'inégalité:

$$
\overline{\overline{\mathcal{Q}}\left(\boldsymbol{S} \dot{+} \overline{\boldsymbol{S}^{*}}\right)} \leqslant 2^{* \alpha} .
$$

D'autre part, le th. $43^{\text {d }}$ entraîne: $S+S^{*} \subset S^{*}+T^{*}$; par application du th. 49 on en conclut que

$$
\overline{\mathcal{Q P}\left(\boldsymbol{S}+\boldsymbol{S}^{*}\right)} \geqslant 2^{* \alpha} \text {. }
$$

Les inégalités $(5)$ et $(6)$ donnent aussitôt:

$$
\overline{\overline{\mathcal{O}\left(S+\boldsymbol{S}^{*}\right)}}=2^{\mathrm{N}_{\alpha}} \text {, c. q. f. } d \text {. }
$$

Le théorème connu, suivant lequel la classe $\boldsymbol{F}$ de tous les ensembles fermés de nombres réels est de la puissance $2^{\mathrm{N}_{0}}$, se laisse déduire facilement da théorème précédent. Soit, en effet, 1 l'ensemble de tous les 
nombres rationnels et $R$ celui de tous les nombres réels (y inclus $+\infty$ et $-\infty)$; posons ensuite $G(x)=1 \cdot E[y<x]$ pour tout $x \in R$. La fonction $G(x)$ transforme noloirement d'une façon biunivoque $R$ en une sous-classe de $\boldsymbol{U}(1)$.Il s'en suit que la fonction $\bar{G}(X)$ transforme de la mème façon la classe $\boldsymbol{U}(R)$ dans une sous-famille de $\boldsymbol{U} \boldsymbol{U}(1) ; \boldsymbol{F}$ étant une sous-classe de $\boldsymbol{U}(R)$, on en conclnt en particulier que $\overline{\bar{F}}=\overline{\bar{E}[X \in \bar{C}]}$. Or, il est aisé de prouver que la formule: $X_{\epsilon} \boldsymbol{F}^{\prime}$ entraîne: $\boldsymbol{S} \bar{G}(X) \subset \bar{G}(X)$ et $\boldsymbol{S}^{*} \bar{G}(X) \subset \overline{G(X)}$, d'où $\bar{G}(X) \in \mathscr{C}(\boldsymbol{S}) \cdot \mathscr{C}\left(\boldsymbol{S}^{*}\right)=\mathscr{C} \mathcal{l}\left(\boldsymbol{S}+\boldsymbol{S}^{*}\right) ;$ on a done $E\left[X \in \mathbb{F}^{\circ}\right] \mathcal{C}$ $\mathcal{C} \mathcal{P}\left(\boldsymbol{S}^{\circ}+\boldsymbol{S}^{*}\right)$, d'où $\overline{\overline{\boldsymbol{H}}} \leqslant \overline{\overline{\mathcal{Q} \ell\left(\boldsymbol{S}+\boldsymbol{S}^{*}\right)}}$. Comme dans le cas considéré $\overline{\overline{1}}=\aleph_{0}$, on en obtient d'après le th. fond. VI: $\overline{\bar{F}} \leqslant 2^{\aleph_{0}}$. D'autre part on a éridemment $\overline{\bar{F}} \geqslant 2^{\mathrm{x}_{0}}$ (puieque p. ex. $\bar{J}(R) \subset \boldsymbol{F}$ et $\overline{\bar{J}(R)}=2^{\mathrm{N}_{0}}$ ); on parvient donc à la formule cherchée: $\overline{\bar{F}}=2^{*_{0}}$, c. q. f. d.

Il est cependant à remarquer que le raisonnement ci-dessus s'appuie d'une façon implicite sur l'axiome du choix, tandis que les autres démonstrations connues du théorème en question sont indépendantes de cet axiome.

Théorème fondamental VII. $S i \overline{\bar{I}}=\aleph_{\alpha}$, on $a \overline{\overline{\mathscr{C P}(\boldsymbol{C}+\bar{S})}}=2^{\alpha_{\alpha}}$.

Démonstration. En tenant compte du th. $42^{\mathrm{a}}$, on obtient par application du th. $28^{\mathrm{b}}: \mathscr{C}(\boldsymbol{C}+\boldsymbol{S})=\mathscr{C}\left(\boldsymbol{C} \stackrel{+}{ }+\boldsymbol{S}^{*}\right)$; à l'aide du th. $21^{\mathrm{b}}$ (pour $\boldsymbol{F}=\boldsymbol{C}$ et $\boldsymbol{G}=\boldsymbol{S}+\boldsymbol{S}^{*}$ ), on en conclut que

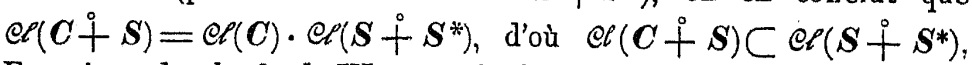
En raison du th. fond. VI, cette inclusion donne:

Posons ensuite

$$
\text { (2) } F(X)=\{0, X, 1-X, 1\} \text { pour tout ensemble } X \text {. }
$$

Soit $a$ un élément arbitraire de 1. $X$ étant un ensemble quelconque de la classe $U(1-\{a\})$, évidemment l'ensemble $1-X$ n'appartient pas à cette classe. Selon (2) il en résulte facilement que les formules: $X \in \boldsymbol{U}(1-\{a\}), Y_{\epsilon} \boldsymbol{U}(1-\{a\})$ et $\boldsymbol{F}(X)=\boldsymbol{F}(Y)$ entrâ̂nent constamment: $X=Y$; en d'autres termes, la fonction $F$ transforme d'une façon biunivoque la classe $U(1-\{a\})$ en son image $\bar{F} U(1-\{a\})$. Par conséquent, on a $\overline{\overline{\bar{F} U(1-\{a\})}}=\overline{\overline{U(1-\{a\})}}$ comme en outre $\overline{\overline{1-\{a\}}}=\aleph_{\alpha}-1=\aleph_{\alpha}$, on en obtient d'après le lem. $10^{\mathbf{a}}$ :

$$
\overline{\overline{\bar{F} \boldsymbol{U}(1-\overline{\{a\})}}}=2^{\mathrm{N} \alpha} \text {. }
$$

Or, on conclut sans peine de (2) à l'aide des déf. 14 et 18 que $\boldsymbol{S} \boldsymbol{F}(X) \subset \boldsymbol{F}(X)$ et $\boldsymbol{C} \boldsymbol{F}(X) \subset \boldsymbol{F}(X)$ pour tout $X$, d'où, en vertu de la déf. 16 et du th. $21^{\mathrm{b}}, \boldsymbol{F}(X) \in \mathcal{O} \mathcal{C}(\boldsymbol{C}) \cdot \mathcal{C} P(S)=\mathscr{C}(\boldsymbol{C}+\boldsymbol{S})$. Il en résulte en particulier que $\bar{F} U(1-\{a\}) \subset \mathcal{C}(C+S)$; en rapprochant cette inclusion de (3), on en obtient:

$$
\overline{\overline{\mathcal{C}}(\boldsymbol{C}+\bar{S})} \geqslant 2^{* \alpha} \text {. }
$$

Les inégalités (1) et (4) donnent finalement:

$$
\overline{\overline{\mathcal{Q}(C)} \overline{+S})}=2^{\alpha_{\alpha}} \text {, c. q. f. d. }
$$

L'analyse des démonstrations des th. fond. V-VII nous condait aux propositions suivantes, qui se rattachent an cas d'ensembles finis:

$$
\begin{aligned}
& S i \overline{\overline{1}}=a<\aleph_{0} \text {, on } a \\
& \text { a) } \overline{\overline{\mathscr{C}(T+S)}}=\overline{\overline{\mathcal{Q} \ell\left(T^{*}+S^{*}\right)}}=2^{a}+1 \text {; } \\
& \text { b) } 2^{a}+1 \leqslant \overline{\overline{e c\left(S+S^{*}\right)}} \leqslant \sum_{r \leqslant a+1}\left(\begin{array}{c}
2^{a} \\
r
\end{array}\right) \text {; }
\end{aligned}
$$

si en outre $\mathfrak{a}>0$, on $a$

$$
\text { c) } 2^{a-1} \leqslant \overline{\overline{\mathscr{e l}\left(\boldsymbol{C}+\boldsymbol{S}^{*}\right)}} \leqslant \sum_{\boldsymbol{r} \leqslant a+1}\left(\begin{array}{c}
2^{a} \\
\boldsymbol{r}
\end{array}\right)
$$

(le symbole $\left(\begin{array}{c}2^{a} \\ \mathfrak{r}\end{array}\right)$ désignant, comme d'habitude, le nombre des combinaisons de $2^{a}$ éléments d̀ $r$ éléments).

Quant aux puissances de $\mathcal{C}(\boldsymbol{S})$ et $\mathcal{C}\left(\boldsymbol{S}^{*}\right)$, on parrient par l'analyse du th. fond. IV anx mêmes délimitations qui ont été indiquées plus haut (p. 232) pour les familles $\mathcal{Q}(\boldsymbol{T})$ et $\mathcal{C} \mathcal{C}\left(\boldsymbol{T}^{*}\right)$.

Les théorèmes fondamentaux établis jusqu'ici épuisent tous les problèmes concernant les puissances des familles de la forme $\mathcal{C}(\boldsymbol{G})$ où $G_{\epsilon} \subseteq\left(\left\{C, T, T^{*}, S, S^{*}\right\}\right)$, c.à-d. des familles des classes closes par rapport à une des opérations $C, T, T^{*}, S$ et $S^{*}$ ou bien par rapport à plusieurs de ces opérations à la fois. Les problèmes non examinés se réduisent facilement à ceux qui ont été examinés explicitement; on a p. ex. en vertu du th. $28 \mathscr{e}(C+S)=\mathcal{C}\left(C+\boldsymbol{C}^{*}\right)=$ $=\mathfrak{e}\left(\boldsymbol{C}+\boldsymbol{S}^{\circ} \boldsymbol{S}^{*}\right)$, en raison du th. $41 \mathcal{O}(\boldsymbol{C}+\boldsymbol{T})=\mathcal{C}(\boldsymbol{C}+\boldsymbol{T}+\boldsymbol{S})=$ $=\mathscr{e}\left(C+\boldsymbol{T}^{*}+\boldsymbol{S}^{*}\right)$ et d'après le th. $43^{\mathrm{d}} \mathscr{C} \mathcal{C}\left(\boldsymbol{T}^{*}\right)=\mathcal{C}_{r}\left(\boldsymbol{T}^{*}+\boldsymbol{S}\right)$. Les résultats acquis peuvent être résumés comme suit: 
A. Chacune des classes $\mathcal{Q}(\boldsymbol{G})$ ò $\boldsymbol{G} \in \mathfrak{S}\left(\left\{\boldsymbol{C}, \boldsymbol{T} ; \boldsymbol{T}^{*}, \boldsymbol{S}, \boldsymbol{S}^{*}\right\}\right)$ coïncide avec une des classes suivantes: $\mathscr{e}(\boldsymbol{C}), \mathcal{C} \mathcal{C}(\boldsymbol{T}), \mathfrak{e}\left(\boldsymbol{T}^{*}\right), \mathcal{C} \mathcal{C}(\boldsymbol{S}), \mathcal{C}\left(\boldsymbol{S}^{*}\right)$, $\mathscr{C}(\boldsymbol{C}+\boldsymbol{T}), \mathcal{C}(\boldsymbol{C}+\boldsymbol{S}), \mathcal{e}(\boldsymbol{T}+\boldsymbol{S}), \quad \mathcal{C}\left(\boldsymbol{T}^{*}+\boldsymbol{S}^{*}\right)$ et $\mathscr{e l}\left(\boldsymbol{S}+\boldsymbol{S}^{*}\right)$.

B. Dans l'hypothèse que $\overline{\bar{I}}=\aleph_{a}$ les familles $\mathscr{e}(\boldsymbol{C}), \mathcal{Q} \mathcal{C}(\boldsymbol{T}), \mathcal{O} \mathcal{L}\left(\boldsymbol{T}^{*}\right)$, $\mathscr{C}(\boldsymbol{S})$ et $\mathscr{C l}\left(\boldsymbol{S}^{*}\right)$ ont la même puissance, à savoir $2^{2^{\alpha} \alpha} ;$ les familles $\left.\mathcal{C}(\boldsymbol{C}+\boldsymbol{S}), \mathcal{Q}_{(} \boldsymbol{T}+\boldsymbol{S}\right), \mathcal{Q}\left(\boldsymbol{T}^{*}+\boldsymbol{S}^{*}\right)$ et $\mathfrak{e}\left(\boldsymbol{S}+\boldsymbol{S}^{*}\right)$ ont aussi la puissance égale, notamment $2^{* \alpha}$; enfin la famille $\mathcal{O}(\boldsymbol{C}+\boldsymbol{T})$ ne contient que deux éléments.

Ces résultats peuvent être étendus à toutes les opérations qui s'obtiennent des opérations considérées à l'aide de l'addition et de la multiplication relative, c.-à-d. aux opérations de la classe $\mathfrak{B}(\{\boldsymbol{C}, \boldsymbol{T}, \boldsymbol{S}\})$. En appliquant notamment le th. B de la p. 217 (§ 3$)$, on constate facilement que toute famille $\mathcal{C}(\boldsymbol{G})$ ou $\boldsymbol{G} \in \mathfrak{B}(\{\boldsymbol{C}, \boldsymbol{T}, \boldsymbol{S}\})$ coïncide soit arec la famille $\mathfrak{C}(\boldsymbol{I})$, soit avec une des familles citées plus haut.

\section{§ 6. Opération $S_{\beta}$. Classes d'ensembles additives au dégré $\beta$.}

L'opération $S_{\beta}$, qui sera traitée dans ce $\S$, consiste à former des ensembles-sommes de moins que $\aleph_{\beta}$ ensembles de la classe donnée $\boldsymbol{K}$. Les classes d'ensembles appartenant à la famille $\mathcal{E}\left(\boldsymbol{S}_{\beta}\right)$ peuvent être appelées classes additives au dégré $\beta$.

La définition exacte de l'opération $S_{\beta}$ est la suivante:

Définition 20. $S_{\beta}(\boldsymbol{K})=\overline{\bar{\Sigma}}\left(\boldsymbol{U}_{\beta}(\boldsymbol{K})-\{0\}\right)$.

Plus simple, mais moins commode serait la définition: $\boldsymbol{S}_{\beta}^{\prime}(\boldsymbol{K})=$ $=\bar{\Sigma} U_{\beta}(\boldsymbol{K})$, resp. $S_{\beta}^{\prime}=\bar{\Sigma} U_{\beta}$ (cf. remarques, p. 233, en relation avec la déf. 18).

Il est aisé d'établir les propriétés suivantes de cette opération:

Théorème 50. a) $S_{\beta} \in \mathfrak{Z}_{\beta}$, donc $S_{\beta} \in \mathfrak{M}$;

b) $I \check{C}^{\circ} S_{\beta}$;

-) $\boldsymbol{S}_{\beta}(0)=0$;

d) $\boldsymbol{S}_{\beta}(\{A\})=\{A\}$

-) $\boldsymbol{S}_{\beta}(\boldsymbol{K}+\{0\})=\boldsymbol{S}_{\beta}(\boldsymbol{K})+\{0\}$

i) $\boldsymbol{S}_{\beta}(\boldsymbol{K}) \leqslant(\overline{\overline{\boldsymbol{K}}})^{\boldsymbol{x}_{\beta}}$.

Démonstration. Les formules $\left.{ }^{a}\right)-0$ ) résultent facilement de la déf. 20 et des considérations du $\S 2$; pour démontrer la formule a) il est commode de s'appuyer sur le lem. $15^{\circ}$.

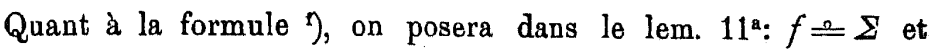
$\boldsymbol{A}=\boldsymbol{U}_{\boldsymbol{\beta}}(\boldsymbol{K})-\{0\}$, d'où, à cause de la déf. $20, \overline{\overline{\boldsymbol{S}_{\beta}(\boldsymbol{K})}} \leqslant \overline{\overline{\boldsymbol{U}_{\beta}(\overline{\boldsymbol{K}})-\{0\}}} \leqslant$ $\leqslant \overline{\overline{\boldsymbol{U}}_{\beta}(\overline{\boldsymbol{K}})}$. Comme en vertu du lem. $10^{\mathrm{c}}: \overline{\overline{\bar{U}_{\beta}(\overline{\boldsymbol{K}})}} \leqslant(\overline{\overline{\boldsymbol{K}}})^{\mathrm{s}}$, on obtient l'inégalité cherchée: $\overline{\overline{\boldsymbol{S}_{\beta}(\boldsymbol{K})}} \leqslant(\overline{\boldsymbol{K}})^{N_{j}}$. Je ne sais pas ériter dans ce raisonnement l'axiome du choix.

Certains rapports entre l'opération $\boldsymbol{S}_{\beta}$ et les opérations examinées dans les $\S \S$ précédents sont données dans le suivant

Théorème 51. a) $T S_{\beta} \stackrel{=}{=} S_{\beta}$;

b) $\boldsymbol{S}_{\beta} \check{\complement}^{\circ} \boldsymbol{S} \check{C}^{\circ} \boldsymbol{T}^{*}$;

-) si $\overline{\overline{\boldsymbol{K}}}<\aleph_{\beta}$ ou bien $\overline{\overline{\boldsymbol{\Sigma}(\overline{\boldsymbol{K}})}}<\aleph_{\beta}$, on a $\boldsymbol{S}_{\beta}(\boldsymbol{K})=\boldsymbol{S}(\boldsymbol{K})$

d) $s i \overline{\overline{1}}<\aleph_{\beta}$, on $a \boldsymbol{S}_{\beta} \doteq \boldsymbol{S}$;

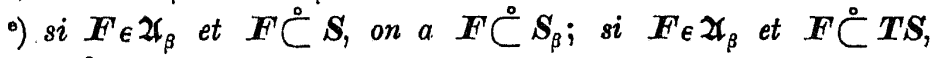
on $a \boldsymbol{F} \dot{\complement} \boldsymbol{T} \boldsymbol{S}_{\beta}$.

Démonstration. Les formules a) et b) se déduisent facilement des déf. 17,18 et 20 et du th. $43^{\text {d }}$; il est cependant à noter que nous ne savons pas démontrer l'inclusion: $\boldsymbol{S}_{\beta} \boldsymbol{T} \subset \boldsymbol{C} \boldsymbol{S}_{\beta}$ sans avoir recours à l'axiome du choix.

Quant à la formule ${ }^{c}$ ), on a évidemment d'après la déf. $5^{\mathrm{a}, \mathrm{b}}$ : $\boldsymbol{U}_{\boldsymbol{\beta}}(\boldsymbol{K})=\boldsymbol{U}(\boldsymbol{K})$ dans le cas où $\overline{\overline{\boldsymbol{K}}}<\boldsymbol{\aleph}_{\beta}$, donc conformément aux déf. 20 et 18: $\boldsymbol{S}_{\beta}(\boldsymbol{K})=\boldsymbol{S}(\boldsymbol{K})$. Dans l'hypothèse que $\overline{\overline{\boldsymbol{\Sigma}(\bar{K})}}<\boldsymbol{N}_{\beta}$ on raisonne par contre comme suit.

Considérons un ensemble arbitraire $X$ tel que

$$
X \in \boldsymbol{S}(\boldsymbol{K}) \text {. }
$$

En raison de la déf. 18, (1) entraîne l'existence d'une classe $\boldsymbol{L}$ vérifiant les formules:

$$
X=\Sigma(\boldsymbol{L})
$$

$$
\boldsymbol{L} \subset \boldsymbol{K} \text { et } \boldsymbol{L} \neq 0 .
$$

De (2) et (3) on conclut en premier lieu que $X \subset \Sigma(K)$, done que $\overline{\bar{X}} \leqslant \overline{\bar{\Sigma}(\overline{\boldsymbol{K}})}$, d'où en vertu de l'hypothèse

$$
\overline{\bar{X}}<\kappa_{\beta} \text {. }
$$

Tenant compte de (2) et appliquant l'axiome du choix, on parvient ensuite à la conclusion qu'on peut faire correspondre à tout 
élément $y$ de $X$ un ensemble $F(y)$ de façon que l'on ait: $y \in F(y) \in L$. Par conséquent cette fonction $F$ remplit les formules suivantes:

et

$$
X \subset \sum_{y \in X} F(y)=\Sigma \bar{F}(X)
$$

$$
\bar{F}(X) \subset \boldsymbol{L}
$$

Il résulte de (6) et (2) que $\Sigma \bar{F}(X) \subset \Sigma(L)=X$; en rapprochant cette inclusion de (5), nous obtenons:

$$
X=\Sigma \bar{F}(X)
$$

Selon (3) et (6) on a $\bar{F}(X) \subset \boldsymbol{K} ;$ le lem. $11^{\text {a }}$ donne en outre $\overline{\overline{\bar{F}(X)}} \leqslant \overline{\bar{X}}$, d'où en vertu de (4) $\overline{\overline{\bar{F}(X)}}<\aleph_{\beta}$. On en conclut que

$$
\bar{F}(X) \epsilon \boldsymbol{U}_{\beta}(\boldsymbol{K}) .
$$

Si $X \neq 0$, on a $\bar{F}(X) \neq 0$, done suivant (8) et $(7) \bar{F}(X) \epsilon \boldsymbol{U}_{\beta}(\boldsymbol{K})-\{0\}$ et $X_{\epsilon} \bar{\Sigma}\left(\boldsymbol{U}_{\beta}(\boldsymbol{K})-\{0\}\right)$, d'où conformément à la déf. 20

(9)

$$
X_{\epsilon} S_{\beta}(\boldsymbol{K}) \text {. }
$$

Or, cette formule (9) se vérifie également dans le cas de $X=0$. On a alors en effet, en raison de (2) et (3), $L=\{0\}$, d'où $\overline{\overline{\boldsymbol{L}}}<\aleph_{\beta}$ et $\boldsymbol{L}_{\epsilon} \boldsymbol{U}_{\beta}(\boldsymbol{K})-\{0\}$, donc, comme auparavant, $X_{\epsilon} \overline{\boldsymbol{\Sigma}}\left(\boldsymbol{U}_{\beta}(\boldsymbol{K})-\{0\}\right)=$ $=S_{\beta}(\boldsymbol{K})$.

Ainsi la formule (1) entraîne constamment (9); par conséquent, la classe $\boldsymbol{K}$ vérifie l'inclüsion: $\boldsymbol{S}(\boldsymbol{K}) \subset \boldsymbol{S}_{\beta}(\boldsymbol{K})$. D'autre part la formule b) du théorème qui nous occupe donne en verta de la déf. $8^{\mathrm{b}}$ : $\boldsymbol{S}_{\beta}(\boldsymbol{K}) \subset \boldsymbol{S}(\boldsymbol{K})$. On a donc finalement:

$$
\boldsymbol{S}_{\beta}(\boldsymbol{K})=\boldsymbol{S}(\boldsymbol{K}) \text { dans l'hypothèse que } \overline{\overline{\boldsymbol{\Sigma}(\bar{K})}}<\aleph_{\beta} \text {, c. q. f. d. }
$$

La formule d) présente une conséquence facile de la proposition établie tout à l'heure. Si, en effet, $\overline{\bar{I}}<\aleph_{\beta}$, on a à plus forte raison $\overline{\overline{\boldsymbol{Z}}(\overline{\boldsymbol{K}})}<\aleph_{\beta}$, done $\boldsymbol{S}_{\beta}(\boldsymbol{K})=\boldsymbol{S}(\boldsymbol{K})$ pour toute classe $\boldsymbol{K}$; ̀̀ l'aide de la déf. $8^{\mathrm{a}}$ on en obtient aussitôt l'identité cherchée: $\boldsymbol{S}_{\boldsymbol{\beta}} \rightleftharpoons \boldsymbol{S}$.

Pour démontrér enfin la proposition $\left.{ }^{\circ}\right)$, envisageons une opération arbitraire $\boldsymbol{F}$ semi-additive au dégré $\beta$ et contenue dana $\boldsymbol{S}$ (c.-d-d. satisfaisant aux formules: $\boldsymbol{F}_{\epsilon} \in \mathfrak{X}_{\beta}$ et $\left.\boldsymbol{F}^{\circ} \complement^{\circ} S\right)$. Conformément an lem. $15^{c}$ on a pour toute classe d'ensembles $\boldsymbol{Y}$ :

$$
F(Y)=\sum_{X_{\epsilon} \sigma_{\beta}(\boldsymbol{Y})} F(X) .
$$

L'application du même lemme à l'opération $\boldsymbol{S}_{\beta}$ donne en raison du th. $50^{\mathrm{a}}$ :

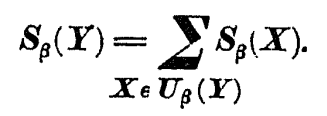

Or, soit $\boldsymbol{X}_{\epsilon} \boldsymbol{U}_{\beta}(\boldsymbol{Y})$, done $\overline{\overline{\boldsymbol{X}}}<\aleph_{\beta}$. En conséquence de la formule d) démontrée auparavant, on obtient: $\boldsymbol{S}_{\beta}(\boldsymbol{X})=\boldsymbol{S}(\boldsymbol{X})$; comme $\boldsymbol{F} \subset \mathcal{C}$, on a en outre $\boldsymbol{F}(\boldsymbol{X}) \subset \boldsymbol{S}(\boldsymbol{X})$, de sorte que

$$
\boldsymbol{F}(\boldsymbol{X}) \subset \boldsymbol{S}_{\beta}(\boldsymbol{X}) \text { pour tout } \boldsymbol{X} \dot{\epsilon} \boldsymbol{V}_{\beta}(\boldsymbol{Y}) \text {. }
$$

Les formules (10)-(12) impliquent aussitôt que $\boldsymbol{F}(\boldsymbol{Y}) \subset \boldsymbol{S}_{\beta}(\boldsymbol{Y})$, quelle que soit la classe $\boldsymbol{Y}$, d'où

$$
\boldsymbol{F} \dot{C} S_{\beta} \text {, c. q. f. d. }
$$

L'opération $\boldsymbol{T} \boldsymbol{S}_{\beta}$ étant, de même que $\boldsymbol{S}_{\beta}$, semi-additive au dégré $\beta$ (d'après le lem. 16 $6^{\mathrm{g}}$ ), la seconde partie de la proposition ${ }^{\circ}$ ) so démontre d'une manière parfaitement analogue.

Je dois la proposition ${ }^{\circ}$ ) dn théorème précédent à MM. Ko źniew$8 k i$ et Lindenbanm ${ }^{1}$ ).

Je vais établir à leur tour certains rapports entre diverses opérations $\boldsymbol{S}_{\beta}$ avec l'indice $\beta$ variable.

Théorème 62. a) Si $A \neq 0$ et $\beta=\sup (\bar{\varphi}(A))$, on a $S_{\beta}=\sum_{x \in A}^{\circ} S_{\phi(x)}$;

b) si $\beta \leqslant \gamma$, on a $\boldsymbol{S}_{\beta} \stackrel{\circ}{\subset} \boldsymbol{S}_{\gamma}$;

c) si $\gamma<\beta$, on a $\boldsymbol{S}_{\beta} \boldsymbol{S}_{\gamma} \stackrel{\text { e }}{=}$;

d) si $\beta \leqslant c f(\gamma)$, on a $\boldsymbol{S}_{\beta} \boldsymbol{S}_{\gamma}=\boldsymbol{S}_{\gamma}$;

e) si $c f(\gamma)<\beta \leqslant \gamma+1$, on a $\boldsymbol{S}_{\beta} \boldsymbol{S}_{\gamma}=\boldsymbol{S}_{\gamma+1}$;

f) si cf $(\beta)=\beta$, on a $S_{\beta}^{2} \rightleftharpoons S_{\beta}$;

g) si c $f(\beta)<\beta$, on a $S_{\beta}^{2} \rightleftharpoons S_{\beta}^{3} \rightleftharpoons S_{\beta+1}$.

1) Cf. a ce propos leur note: Sur les opérations d'addition et de multiplication dans les classes d'ensembles, Fund. Math. $\mathrm{XV}, \mathrm{p}$. 343, Th. 1. 
Démonstration. La formule a) constitue une conséquence facile des déf. 20 et $9^{b}$, puisqu'on a en raison de la déf. $5^{b}$ $\boldsymbol{U}_{\beta}(\boldsymbol{K})=\sum_{x \in A} \boldsymbol{U}_{\varphi(x)}(\boldsymbol{K})$ dans l'hypothèse que $\beta=\sup (\bar{\varphi}(A))$. Si l'on pose en particulier: $\beta=\gamma, A=\{0,1\}$ et $\varphi(0)=\beta \leqslant \varphi(1)=\gamma$, on obtient de a): $\boldsymbol{S}_{\gamma} \rightleftharpoons \boldsymbol{S}_{\beta}+\boldsymbol{S}_{\gamma}$ et on parvient ainsi à l'inclusion ${ }^{\mathrm{b}}$ ).

c) Suivant le th. $50^{\mathrm{a}, \mathrm{b}}$, on a $\boldsymbol{S}_{\beta} \in \mathfrak{M}$ et $\boldsymbol{I} \subset \boldsymbol{S}_{\gamma}$; en appliquant donc la seconde partie du lem. $17^{\circ}$ (pour $\boldsymbol{F} \rightleftharpoons \boldsymbol{S}_{\gamma}$ et $\boldsymbol{G} \rightleftharpoons \boldsymbol{S}_{\beta}$ ), on en obtient:

$$
S_{\beta} \stackrel{\circ}{\complement} S_{\beta} S_{\gamma} .
$$

Posons dans le lem. 16: $F \bumpeq S_{\beta}$ et $G=S_{\gamma}$; comme d'après le th. $50^{\mathrm{a}} \quad \boldsymbol{S}_{\beta} \in \mathfrak{A}_{\beta}$ et $\boldsymbol{S}_{\gamma} \in \mathfrak{X}_{\gamma}$ et comme par hypothèse $\gamma<\beta$, ce lemme donne:

$$
\boldsymbol{S}_{\beta} \boldsymbol{S}_{\gamma} \in \mathfrak{X}_{\beta} .
$$

Les formules: $\boldsymbol{S}_{\beta} \in \mathfrak{M}$ et $\boldsymbol{S}_{\gamma} \subset \mathbf{C}$ (th. $50^{\mathrm{a}}$, th. $51^{\mathrm{b}}$ ) impliquent ensuite, conformément à la déf. $12^{\mathrm{a}}: S_{\beta} S_{\gamma} \check{\complement}^{\circ} S_{\beta} S$; comme d'autre part $I \check{C}^{\circ} S_{\beta} \subset S=S^{2}$ (th. $50^{\mathrm{a}}$, th. $51^{\mathrm{b}}$, th. $42^{\mathrm{b}}$ ), on obtient à l'aide du lem. $17^{\mathrm{d}}: S_{\beta} S=S$. Par conséquent,

$$
\text { - } \boldsymbol{S}_{\beta} \boldsymbol{S}_{\gamma} \stackrel{\circ}{\complement} \boldsymbol{S} \text {. }
$$

Or, d'après le th. 51• (pour $F=S_{\beta} S_{\gamma}$ ), les formules (2) et (3) entraînent l'inclusion: $S_{\beta} S_{\gamma} \stackrel{\circ}{\complement} S_{\beta}$. qui, rapprochée de (1), donne finalement:

$$
S_{\beta} S_{\gamma} \rightleftharpoons S_{\beta} \text {, c. q. f. d. }
$$

La formule d) s'obtient d'une façon complètement analogue.

Passons à la proposition ${ }^{\circ}$ ); admettons done que $c f(\gamma)<\beta \leqslant$ $\leqslant \gamma+1$. En vertu de la formule $\left.{ }^{b}\right)$ établie auparavant on a dans ce cas $\boldsymbol{S}_{\beta} \stackrel{\circ}{\subset} \boldsymbol{S}_{\gamma+1}$, d'où suivant le lem. $14^{\mathrm{b}} \boldsymbol{S}_{\beta} \boldsymbol{S}_{\gamma} \check{\complement}^{\circ} \boldsymbol{S}_{\gamma+1} \boldsymbol{S}_{\gamma}$; si l'on pose d'autre part: $\beta=\gamma+1$ dans la formule $\%$, on obtient: $\boldsymbol{S}_{\gamma+1} \boldsymbol{S}_{\gamma} \rightleftharpoons \boldsymbol{S}_{\gamma+1}$. Par conséquent,

$$
\text { (14) } \quad \boldsymbol{S}_{\beta} \boldsymbol{S}_{\gamma} \stackrel{\dot{C}}{\complement} \boldsymbol{S}_{\gamma+1} \text {, lorsque } \beta \leqslant \gamma+1 \text {. }
$$

Soient $\boldsymbol{K}$ une classe d'ensembles arbitraire et $X$ un ensemble tel que

$$
X \in \boldsymbol{S}_{\gamma+1}(\boldsymbol{K})
$$

Conformément aux déf. 20 et $5^{\text {b }}$, (15) entraîne l'existence d'une. classe $\boldsymbol{L}$ satisfaisant aux conditionis:

$$
\begin{gathered}
X=\Sigma(\boldsymbol{L}), \\
\boldsymbol{L} \subset \boldsymbol{K}, \overline{\overline{\boldsymbol{L}}}<\aleph_{\gamma+1} \text { et } \boldsymbol{L} \neq 0 .
\end{gathered}
$$

Il résulte de (17) que $0<\overline{\bar{L}} \leqslant \aleph_{\gamma}$. A l'aide du lem. $2^{\text {b }}$ (pour $A=\boldsymbol{L}$ et $\alpha=\gamma$ ) on en conclut qu'il existe une suite de classes $\boldsymbol{L}_{\boldsymbol{\xi}}$ du type $\omega_{c(\gamma)}$ vérifiant les formules:

$$
\begin{gathered}
\boldsymbol{L}=\sum_{\xi<\omega_{c f(\gamma)}} \boldsymbol{L}_{\xi} ; \\
\overline{\boldsymbol{L}}_{\xi}<\kappa_{\gamma} \text { et } \boldsymbol{L}_{\xi} \neq 0 \text { pour } \xi<\omega_{(f) \gamma) .}
\end{gathered}
$$

Les formules (16) et (18) donnent, en raison de la loi associative de l'addition des ensembles:

(20) $\quad X=\Sigma\left(\sum_{\xi<\omega_{c f(\gamma)}} L_{\xi}\right)=\sum_{\xi<\omega_{c f(\gamma)}} \Sigma\left(L_{\xi}\right)=\Sigma\left(\underset{\Sigma\left(L_{\xi}\right)}{E}\left[\xi<\omega_{c f(\gamma)}\right]\right)$.

En vertu de (17) et (18), $\boldsymbol{L}_{\xi} \subset \boldsymbol{K}$ pour $\xi<\omega_{c f(y))}$, d'où, selon (19), $\boldsymbol{L}_{\boldsymbol{\xi}} \in \boldsymbol{U}_{\gamma}(\boldsymbol{K})-\{0\}$. Par conséquent, $\boldsymbol{\Sigma}\left(\boldsymbol{L}_{\xi}\right) \in \overline{\boldsymbol{\Sigma}}\left(\boldsymbol{U}_{\boldsymbol{\gamma}}(\boldsymbol{K})-\{0\}\right.$, donc conformément à la déf. 20

$$
\Sigma\left(\boldsymbol{L}_{\xi}\right) \in S_{\gamma}(\boldsymbol{K}) \text { pour } \xi<\omega_{c f(\gamma)} \text {. }
$$

Posons momentanément: $\quad \Sigma\left(\boldsymbol{L}_{\xi}\right)=F^{\prime}(\xi)$ d'où $\left.\underset{\Sigma\left(\boldsymbol{I}_{\xi}\right)}{E\left[\xi<\omega_{\text {rffy }}\right]}\right]=$ $=\bar{F}\left(\underset{\xi}{E}\left[\xi<\omega_{c f(\gamma)]}\right]\right)$ Par l'application du lem. 11 $1^{\mathrm{a}}$ (pour $f=F$ et $\left.A=\underset{\xi}{E}\left[\xi<\omega_{f f(\gamma)}\right]\right)$ nous en obtenons: $\overline{\overline{\Sigma\left(L_{\xi}\right)}} \overline{\overline{E\left[\xi<\omega_{c f(\gamma)}\right]}} \leqslant \overline{\overline{E\left[\xi<\omega_{c f(\gamma)}\right]}}=$

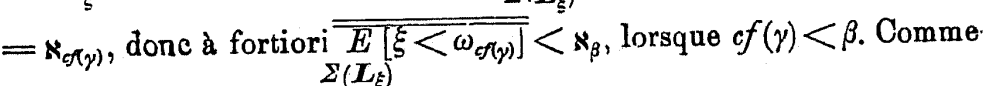

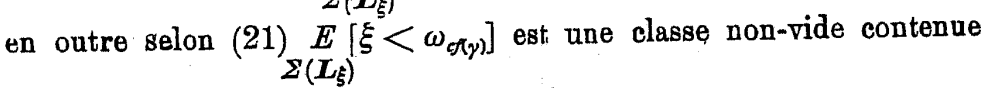
dans $\boldsymbol{S}_{\gamma}(\boldsymbol{K})$, on conclut de la déf. $5^{\mathrm{b}}$ que

(22) $\underset{\Sigma\left(\boldsymbol{I}_{\xi}\right)}{E}\left[\xi<\omega_{c \kappa(\gamma)}\right] \in \boldsymbol{U}, s_{\gamma}(\boldsymbol{K})-\{0\}$ pour $c f(\gamma)<\beta$.

Il résulte de (20) et (22) que $X_{\epsilon} \overline{\boldsymbol{\Sigma}}\left(\boldsymbol{U}_{\beta} \boldsymbol{S}_{\gamma}(\boldsymbol{K})-\{0\}\right)$, d'où suivant la déf. 20

$$
X \in \boldsymbol{S}_{\beta} \boldsymbol{S}_{\gamma}(\boldsymbol{K}), \text { lorsque of }(\gamma)<\beta .
$$


Ainsi la formule (15) entraîne toujours (23). Toute classe d'ensembles $\boldsymbol{K}$ vérifie donc l'inclusion: $\boldsymbol{S}_{\gamma+1}(\boldsymbol{K}) \subset \boldsymbol{S}_{\beta} \boldsymbol{S}_{\gamma}(\boldsymbol{K})$; autrement dit,

$$
\boldsymbol{S}_{\gamma+1} \stackrel{\circ}{\complement} \boldsymbol{S}_{\beta} \boldsymbol{S}_{\gamma}, \text { lorsque of }(\gamma)<\beta .
$$

Les formules (14) et (24) donnent aussitôt:

$$
\boldsymbol{S}_{\beta} \boldsymbol{S}_{\gamma} \rightleftharpoons \boldsymbol{S}_{\gamma+1} \text {, lorsque of }(\gamma)<\beta \leqslant \gamma+1 \text {, c. q. f. d. }
$$

Tenant compte de la déf. $10^{\mathrm{a}, \mathrm{b}, \mathrm{c}}$, on obtient comme un cas particulier de la formule d) (pour $\gamma=\beta$ ) la formule ${ }^{\mathrm{t}}$ ): $\boldsymbol{S}_{\beta} \bumpeq \boldsymbol{S}_{\beta}$, lorsque $c f(\beta)=\beta$.

En posant de même dans la formule $\left.{ }^{a}\right): \gamma=\beta$, on parvient à l'égalité: $\boldsymbol{S}_{\beta}^{2}=\boldsymbol{S}_{\beta+1}^{-}$, lorsque of $(\beta)<\beta$, d'où $\boldsymbol{S}_{\beta}^{3}=\boldsymbol{S}_{\beta}^{2} \boldsymbol{S}_{\beta} \rightleftharpoons \boldsymbol{S}_{\beta+1} \boldsymbol{S}_{\beta}$; comme en outre, d'après la formule ${ }^{\circ}$ ), $\boldsymbol{S}_{\beta+1} \boldsymbol{S}_{\beta}=\boldsymbol{S}_{\beta+1}$, on obtient finalement $\mathfrak{s}): S_{\beta}^{2}=S_{\beta}^{3}=S_{\beta+1}$, lorsque of $(\beta)<\beta$.

La démonstration da th. 52 est ainsi achevée.

On aperçoit facilement l'analogie entre le lem. $8^{\mathrm{a}, \mathrm{b}, \mathrm{c}}$ et le th. $52^{\mathrm{e}, \mathrm{d}, \boldsymbol{\theta}}$ : l'opération $\boldsymbol{S}_{\beta} \boldsymbol{S}_{\gamma}$ coïncide respectivement avec une des opérations $\boldsymbol{S}_{\beta}$ $\boldsymbol{S}_{\gamma}$ ou $\boldsymbol{S}_{\gamma+1}$ dans les mêmes hypothèses, dans lesquelles le nombre

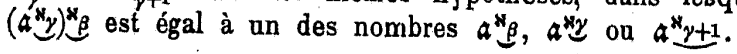

A même titre que l'opération $S_{\beta}$, son opération double $S_{\beta}^{*}$ va nous intéresser ici; il est parfois commode d'assigner à cette opération un symbole indépendant, p. ex. $\boldsymbol{P}_{\beta}$. En s'appuyant sur les déf. 14,15 et 20 , on peut établir le théorème suivant qui explique le sens de l'opération $\boldsymbol{S}_{\beta}^{*}$ :

Théorème 53. $S_{\beta}^{*}(\boldsymbol{K})=\bar{\Pi}\left(U_{\beta}(\boldsymbol{K})-\{0\}\right)$.

Comme on voit, l'opération en question consiste à former des ensembles-produits de moins que $\aleph_{\beta}$ ensembles de la classe donnée $\boldsymbol{K}$ Les classes d'ensembles clọses par rapport à l'opération $\boldsymbol{S}_{\beta}^{*}$ peuvent être appelées classes multiplicatives au dégré $\beta$,

En s'appuyant sur le lem. 19 on peut déduire des propriétés de l'opération $S_{\beta}$ qui viennent d'être établies dans les th. 50-52 celles qui leur correspondent pour l'opération $S_{\beta}^{*}$. Ce ne sont que les propriétés suivantes (correspondant aux th. $50^{\mathrm{d}, \mathrm{e}}$ et $51^{\mathrm{c}}$ ) qui ne s'en obtiennent pas automatiquement, mais exigent une démonstration
spéciale:
Théorème 54. a) $S_{\beta}^{*}(\{A\})=\{A\}$;

b) $\boldsymbol{S}_{\beta}^{*}(\boldsymbol{K}+\{0\})=\boldsymbol{S}_{\beta}^{*}(\boldsymbol{K})+\{0\}$;

e) si $\overline{\bar{K}}<\aleph_{\beta}$ ou bien $\overline{\overline{\Sigma(K)}}<\aleph_{\beta}$, on a $S_{\beta}^{*}(\boldsymbol{K})=S^{*}(\boldsymbol{K})$.

Démonstration des formules a) et ${ }^{b}$ ) est tout à fait élémentaire. Pour établir la proposition ${ }^{c}$ ), il est commode d'envisager la classe $\boldsymbol{K}^{\times}=\underset{\Sigma(\boldsymbol{K})-X}{E}[X \in \boldsymbol{K}]$. On a évidemment $\overline{\overline{\boldsymbol{K}} \times}<\aleph_{\beta}$ ou bien $\overline{\overline{\boldsymbol{\Sigma}\left(\boldsymbol{K}^{\mathrm{X}}\right)}}<\aleph_{\beta}$ (suivant l'hypothèse admise pour la classe $\boldsymbol{K}$ ), d'où d'après le th. 51 ${ }^{\circ} \boldsymbol{S}_{\beta}\left(\boldsymbol{K}^{\times}\right)=\boldsymbol{S}\left(\boldsymbol{K}^{\times}\right)$; d'autre part, en rapprochant les déf. 20 et 18 des th. 53 et 44 , on se convaint facilement que $\boldsymbol{S}_{\beta}^{*}(\boldsymbol{K})=\underset{\Sigma(\boldsymbol{K})-X}{E}\left[X_{\in} \in \boldsymbol{S}_{\beta}\left(\boldsymbol{K}^{\times}\right)\right]$et de nềme que $\boldsymbol{S}^{*}(\boldsymbol{K})=$ $\left.=E\left[X \in S\left(\boldsymbol{K}^{\times}\right)\right]^{1}\right)$. On en obtient aussitôt la formule cherchée: $\boldsymbol{S}_{\beta}^{*}(\boldsymbol{K})=\boldsymbol{S}^{*}(\boldsymbol{K})$.

En dehors des propriétés de $\boldsymbol{S}_{\beta}$ et $\boldsymbol{S}_{\beta}^{*}$ énoncées dans les derniers théorèmes les propriétés suivantes sont à mentionner ici. On a le théorème: pour que $\boldsymbol{F} \in \mathfrak{Z}_{\beta}$ et $\boldsymbol{F}(0)=0$, il faut et il suffit que $\boldsymbol{F} \boldsymbol{\Sigma}=\boldsymbol{\Sigma} \overline{\boldsymbol{F}} \boldsymbol{S}_{\beta}$ (c.-̀̀̀-d. que $\boldsymbol{F} \boldsymbol{\Sigma}(\mathscr{d} \mathfrak{i})=\sum \boldsymbol{F}(\boldsymbol{X})$, quelle que soit la $\boldsymbol{X}_{\epsilon} \boldsymbol{S}_{\beta}(\mathscr{O H})$

famille de classes $\mathscr{O}$; cf. le théoréme analogue, p. 240); donc en particulier: $S_{\beta}=\Sigma \bar{S}_{\beta} S_{\beta}$. On a ensuite les formules: $\Sigma S_{\beta} \rightleftharpoons \Sigma=\Sigma S_{\beta}^{*}$

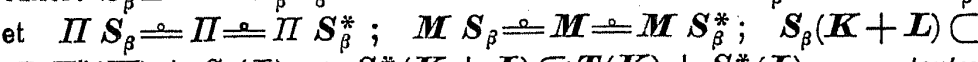
$C \boldsymbol{T}^{*}(\boldsymbol{K})+\boldsymbol{S}_{\beta}(\boldsymbol{L})$ et $\boldsymbol{S}_{\beta}^{*}(\boldsymbol{K}+\boldsymbol{L}) \boldsymbol{C}(\boldsymbol{K})+\boldsymbol{S}_{\beta}^{*}(\boldsymbol{L})$ pour toutes deux classes $\boldsymbol{K}$ et $\boldsymbol{L}$ (resp. $\left.\boldsymbol{S}_{\beta} \mid \boldsymbol{F}+\boldsymbol{G}\right] \complement^{\circ} \boldsymbol{T}^{*} \boldsymbol{F}+\boldsymbol{S}_{\beta} \boldsymbol{G}$ et $\boldsymbol{S}_{\beta}^{*}[\boldsymbol{F}+\boldsymbol{G}] \check{C}^{\circ}$ $\stackrel{\circ}{C} \boldsymbol{F}+\boldsymbol{S}_{\beta}^{*} \boldsymbol{G}$ pour toutes deux opérations $\boldsymbol{F}$ et $\left.\boldsymbol{G}\right)$. On peut établir les réciproques da th. $52^{s, g}$ : les formules: $\boldsymbol{S}_{\beta}^{g} \rightleftharpoons \boldsymbol{S}_{\beta}$ et $\operatorname{cf}(\beta)=\beta$ sont donc équivalentes, de même que les formules: $\boldsymbol{S}_{\beta}^{2} \stackrel{\rho}{=} \boldsymbol{S}_{\beta+1}$ et $\operatorname{cf}(\beta)<\beta$. Il existe enfin certaines relations moins simples entre les opérations de la forme $\boldsymbol{S}_{\beta} \boldsymbol{S}_{\gamma}^{*}, \boldsymbol{S}_{\beta} \boldsymbol{S}_{\gamma}^{*} \boldsymbol{S}_{\delta} \ldots$ d'une part et celles de la forme $\boldsymbol{S}_{\beta}^{*} \boldsymbol{S}_{\gamma}$, $\boldsymbol{S}_{\beta}^{*} \boldsymbol{S}_{\gamma} \boldsymbol{S}_{\delta}^{*} \ldots$ d'autre part $\left.{ }^{3}\right)$

Les opérations $\boldsymbol{S}_{\beta}$ et $\boldsymbol{S}_{\beta}^{*}$ n'étaient pas étudieés jusqu'à présent que

1) Cf. les remarques, p. 211, sur la coïncidence des opérations $F^{*}$ et $F^{\times}$pour $\boldsymbol{F} \bumpeq \boldsymbol{S}$, resp. $\boldsymbol{F} \bumpeq \boldsymbol{S}_{\beta}$.

2). Cf. ì ce propos: W. Sierpinski et A. Tarski, Sur une propriété caractéristique des nombres inuccessibles, Fund. Math. XV, p. 292; A. K oźniewski et A. Lindenbanm, Sur les opérations d'addition et de multiplication dans les classes d'ensembles, ibid. p. 342 
dans les cas particuliers de $\beta=0$ et $\beta=11$ ). M. Hausdorff a introduit les symboles spécianx pour les opérations $S_{1}$ et $S_{1}^{*}$, à savoir $\sigma$ et $\delta$ $\left(\boldsymbol{K}_{\sigma}=\boldsymbol{S}_{1}(\boldsymbol{K}), \boldsymbol{K}_{\delta}=\boldsymbol{S}_{1}^{*}(\boldsymbol{K})\right)$, ainsi que les termes spécianx pour les classes d'ensembles appartenant respectivement aux familles $\mathscr{C}\left(\boldsymbol{S}_{0}+\boldsymbol{S}_{0}^{*}\right)$, $\mathscr{C}\left(\boldsymbol{S}_{1}\right), \mathcal{C} \mathcal{P}\left(\boldsymbol{S}_{1}^{*}\right)$ et $\mathcal{C}\left(\boldsymbol{S}_{1}+\boldsymbol{S}_{1}^{*}\right)$, à savoir Ring, $\sigma$ System, $\delta$-System et $(\sigma \delta)-$ System $\left.^{2}\right)$.

Pour les considérations qui vont suivre, il est commode d'intro-

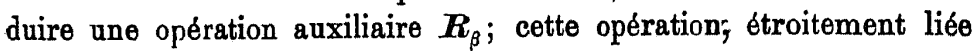
avec $\boldsymbol{S}_{\beta}$ et $\boldsymbol{S}_{\beta}^{*}$, semble d'ailleurs présenter par elle-méme quelque intérêt. Afin d'effectuer l'opération $\boldsymbol{R}_{\beta}$ sur une classe d'ensembles donnée $\boldsymbol{K}$, on envisage toutes les sous-classés $\boldsymbol{X}$ de $\boldsymbol{K}$ qui contiennent moins de $\aleph_{\beta}$ ensembles et on forme pour tout $\boldsymbol{X}$ toutes les sommes des produits (ou bien, ce qui revient au-même d'après le th. 47 , tous les produits des sommes) d'ensembles de $\boldsymbol{X}$; les ensembles ainsi formés constituent la classe $\boldsymbol{R}_{\beta}(\boldsymbol{K})$. On a donc en symboles:

$$
\text { Définition 21. } R_{\beta}(K)=\sum_{X_{\varepsilon} U_{\beta}(K)} S S^{*}(X) .
$$

On peat mettre la définition précédente dans une forme plus concise, à savoir : $\boldsymbol{R}_{\beta}(\boldsymbol{K})={ }^{\circ} \overline{\boldsymbol{S S ^ { * }}} \boldsymbol{U}_{\beta}(\boldsymbol{K})$, resp. $\boldsymbol{R}_{\beta}=\boldsymbol{\Sigma} \overline{\boldsymbol{S S ^ { * }}} \boldsymbol{U}_{\beta}$. Il est à noter que les opérations $\boldsymbol{S}_{\beta}$ et $\boldsymbol{S}_{\beta}^{*}$ se laissent exprimer aussi par des formules analogues: $\boldsymbol{S}_{\beta} \varrho \boldsymbol{\Sigma} \overline{\boldsymbol{S}} \boldsymbol{U}_{\beta}$ et $\boldsymbol{S}_{\beta}^{*} \supseteq \boldsymbol{\Sigma} \overline{\boldsymbol{S}^{*}} \boldsymbol{U}_{\beta}$. Ce procédé de former les opérations $\boldsymbol{S}_{\beta}, \boldsymbol{S}_{\beta}^{*}$ et $\boldsymbol{R}_{\beta}$ à partir de $\boldsymbol{S}, \boldsymbol{S}^{*}$ et $\boldsymbol{S} \boldsymbol{S}^{*}$ se prête à une généralisation, qui consiste à faire correspondre à toute opération $\boldsymbol{F}$ et à tout nombre ordinal $\beta$ une opération $\boldsymbol{F}_{\beta}$ déterminée par la formule: $\boldsymbol{F}_{\beta}=\boldsymbol{\Sigma} \overline{\boldsymbol{F}} \boldsymbol{U}_{\beta} ;$ il s'y rattache aussi l'opération plus vaste $\boldsymbol{F}_{\infty}=\bar{\Sigma} \overline{\boldsymbol{F}} \boldsymbol{U}$ (qui ne constitue d'ailleurs qu'un cas particulier de $\boldsymbol{F}_{\beta}$ ). L'étude de ces notions, qui rentrent dans l'algorythme esquissé an $\S 2$, ne sera pas développée ici.

Certaines propriétés élémentaires de l'opération $\boldsymbol{R}_{\beta}$ sont données dans les deux théorèmes saivants:

1) Le cas de $\beta=2$ a été envisagé par M. Sierpiński dans son article: Súr les ensembles hyperboreliens, Comptes rendus des ś́ances de la Soc. des Sc. et des L. de Varsovie 1926, p. 16-22.

2) Cf. F. Ha usd orff, Mengenlehre II, Aufl, Berlin und Leipzig 1927, p. 77-85; cf. aussi W. Sierpiński, Wstepp do teorji mnogosici $i$ topologji (Introduction à la Theorie des Ensembles et à la Topologie, on polonais), Lẃ́bw 1930, p. 108-119.
Théorème 55. a) $\boldsymbol{R}_{\beta} \in \mathfrak{I}_{\beta}$, donc $\boldsymbol{R}_{\beta} \in \mathfrak{M}$;

b) $\boldsymbol{I} \subset \boldsymbol{R}_{\beta}$

c) si $\boldsymbol{F}_{\in} \in \mathfrak{Z}_{\beta}$ et $\boldsymbol{F}^{\circ} \subset \boldsymbol{S} S^{*}$, on a $\boldsymbol{F}^{\circ} \boldsymbol{R}_{\beta}$;

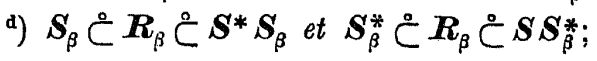

o) $\boldsymbol{R}_{\beta}^{*}=\boldsymbol{R}_{\beta}$ et $\boldsymbol{C} \boldsymbol{R}_{\beta}=\boldsymbol{R}_{\beta} \boldsymbol{C}$

1) $\boldsymbol{R}_{\beta}(\boldsymbol{K}+\boldsymbol{L}) \subset \boldsymbol{T} \boldsymbol{S}_{\beta}(\boldsymbol{K})+\boldsymbol{T}^{*} \boldsymbol{S}_{\beta}^{*}(\boldsymbol{L})$ pour toutes doux classes d'etsembles $\boldsymbol{K}$ et $\boldsymbol{L}$ (en d'autres termes, $\boldsymbol{R}_{\beta}[\boldsymbol{F}+\boldsymbol{G}] \mathcal{C}^{\circ} \boldsymbol{T} \boldsymbol{S}_{\beta}^{*} \boldsymbol{F}+\boldsymbol{T}^{*} \boldsymbol{S}_{\beta}^{*} \boldsymbol{G}$ pour toutes deux opérations $\boldsymbol{F}$ et $\boldsymbol{G})$;

g) $\boldsymbol{R}_{\beta}(\boldsymbol{K}) \leqslant(\overline{\overline{\boldsymbol{K}}})^{\alpha_{\beta}} \cdot 2^{2{ }_{\beta}}$.

Démonstration. Les formules ${ }^{a}$ ) et ${ }^{b}$ ) se déduisent facilement de la déf. 21 et du th. $42^{b}$ à l'aide des définitions et des lemmes du $\S 2$.

La démonstration de ${ }^{\circ}$ ) est analogue à celle du th. $51^{\circ}$.

Si l'on pose dans $\left.{ }^{\circ}\right): F=S_{\beta}$, resp. $\boldsymbol{F}^{\boldsymbol{F}} \bumpeq \boldsymbol{S}_{\beta}^{*}$, et si l'on tient compte des formules: $\boldsymbol{S}_{\beta} \in \mathfrak{Z}_{\beta}$ et $\boldsymbol{S}_{\beta} \stackrel{\complement}{\complement} \boldsymbol{S} \boldsymbol{S}^{*}$, resp. $\boldsymbol{S}_{\beta}^{*} \in \mathfrak{Z}_{\beta}$ et $\boldsymbol{S}_{\beta}^{*} \mathcal{\complement} \boldsymbol{S} \boldsymbol{S}^{*}$ (qui s'obtiennent sans peine des th. $50^{\mathrm{a}}, 51^{\mathrm{b}}$ et $42^{\mathrm{a}, \mathrm{b}}$ par l'application des lem. $17^{\circ}$ et $\left.19^{c, j, 1}\right)$, on parvient aussitôt aux inclusions: $\boldsymbol{S}_{\beta} \stackrel{\circ}{\complement}^{\circ} \boldsymbol{R}_{\beta}$ et $\boldsymbol{S}_{\beta}^{*} \check{\complement}^{\circ} \boldsymbol{R}_{\beta}$. En appliquant d'autre part les th. $47,51^{\mathrm{c}}$ et $54^{\mathrm{c}}$, on obtient les transformations suivantes de la déf. 21: $\boldsymbol{R}_{\beta}(\boldsymbol{K})=$ $=\sum_{X \in V_{B}(\boldsymbol{K})} S^{*} \boldsymbol{S}(\boldsymbol{X})=\sum_{\boldsymbol{X} \in \bar{V}_{\beta}(\boldsymbol{K})} \boldsymbol{S}^{*} \boldsymbol{S}_{\beta}(\boldsymbol{X})=\sum_{\boldsymbol{X}_{\in} \boldsymbol{U}_{\beta}(\boldsymbol{K})} \boldsymbol{S} \boldsymbol{S}_{\beta}^{*}(\boldsymbol{X}) ;$ or, les opérations $\boldsymbol{S}^{*} \boldsymbol{S}_{\beta}$ et $\boldsymbol{S} \boldsymbol{S}_{\beta}^{*}$ étant monotones (d'après les th. $42^{\mathrm{a}}, 50^{\mathrm{a}}$ et les lem. $\left.19 \mathrm{~g}, 16^{\mathrm{c}}\right)$, on en conclut à l'aide du lem. $15^{\mathrm{a}}$ que $\boldsymbol{R}_{\beta}(\boldsymbol{K}) \subset \boldsymbol{S}^{*} \boldsymbol{S}_{\beta}(\boldsymbol{K})$ et $\boldsymbol{R}_{\beta}(\boldsymbol{K}) \subset \boldsymbol{S} \boldsymbol{S}_{\beta}^{*}(\boldsymbol{K})$ pour toute classe $\boldsymbol{K}$, donc que $\boldsymbol{R}_{\beta} \stackrel{\circ}{\complement} \boldsymbol{S}^{*} \boldsymbol{S}_{\beta}$ et $\boldsymbol{R}_{\beta} \stackrel{\circ}{\complement} \boldsymbol{S} \boldsymbol{S}_{\beta}^{*}$. Les formules d) sont ainsi entièrement établies.

Pour démontrer les identitès ${ }^{\theta}$ ), observons qu'en vertu de a) et du lem. $19^{\mathrm{i}}$ on a $\boldsymbol{R}_{\beta}^{*} \in \mathfrak{Z}_{\beta}$; comme d'autre part $\boldsymbol{S} \boldsymbol{S}_{\beta}^{*} \stackrel{\circ}{\complement}^{\circ} \boldsymbol{S}^{*}$ (th. $51^{\mathrm{b}}$, lem. $19^{\mathrm{c}}$, th. $42^{\mathrm{a}}$, déf. $\left.12^{\mathrm{a}}\right)$, on déduit de d) à l'aide du lem. $19^{\mathrm{b}, \mathrm{c}_{0}}$ :

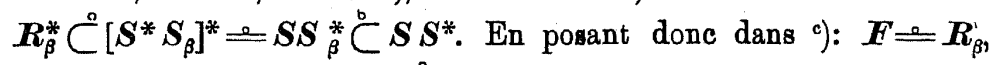
nous en concluons que $\boldsymbol{R}_{\beta}^{*} \stackrel{\circ}{\complement}^{\circ} \boldsymbol{R}_{\beta}$, d'où par l'application du lem. $19^{\mathrm{b}, \mathrm{c}}$ : $\boldsymbol{R}_{\beta} \rightleftharpoons\left[\boldsymbol{R}_{\beta}^{*}\right]^{*} \stackrel{\circ}{C}^{\boldsymbol{R}_{\beta}^{*}}$ et finalement: $\boldsymbol{R}_{\beta}^{*} \varrho \boldsymbol{R}_{\beta}$. En raison du lem. 19a, cette dernière formule donne en ontre: $\boldsymbol{C} \boldsymbol{R}_{\beta}=\boldsymbol{R}_{\beta} \boldsymbol{C}$, c. q. f. d.

En ce qui concerne ${ }^{f}$ ), on raisonnera comme il suit.

Envisageons une classe d'ensembles $\boldsymbol{X}$ telle que
(1)

$$
\boldsymbol{X}_{\epsilon} \boldsymbol{U}_{\boldsymbol{\beta}}(\boldsymbol{K}+\boldsymbol{L})
$$


Conformément à la déf. $5^{\mathrm{b}}$ on a donc $\boldsymbol{X} \subset \boldsymbol{K}+\boldsymbol{L}$ et $\overline{\boldsymbol{X}}<\boldsymbol{N}_{\beta}$ r d'où

$$
\begin{gathered}
\boldsymbol{X}=\boldsymbol{K} \cdot \boldsymbol{X}+\boldsymbol{I} \cdot \boldsymbol{X} \\
\overline{\overline{\boldsymbol{K}} \cdot \overline{\boldsymbol{X}}}<\aleph_{\beta} \text { et } \overline{\overline{\boldsymbol{L} \cdot \boldsymbol{X}}}<\aleph_{\beta} .
\end{gathered}
$$

En appliquant le th. $45^{\text {c }}$, on obtient de $(2): \boldsymbol{S}^{*}(\boldsymbol{X}) \subset \boldsymbol{T}(\boldsymbol{K} \cdot \boldsymbol{X})+$ $+S^{*}(\boldsymbol{L} \cdot \boldsymbol{X})$; l'opération $S$ étant monotone (th. $\left.42^{\mathrm{a}}\right)$, il s'en suit que $\boldsymbol{S} \boldsymbol{S}^{*}(\boldsymbol{X}) \subset \boldsymbol{S}\left(\boldsymbol{T}(\boldsymbol{K} \cdot \boldsymbol{X})+\boldsymbol{S}^{*}(\boldsymbol{L} \cdot \boldsymbol{X})\right)$. D'autre part, le th. $43^{\circ}$ pour $\boldsymbol{K}=\boldsymbol{S}^{*}(\boldsymbol{L} \cdot \boldsymbol{X})$ et $\boldsymbol{L}=\boldsymbol{T}(\boldsymbol{K} \cdot \boldsymbol{X})$ donne: $\boldsymbol{S}(\boldsymbol{T}(\boldsymbol{K} \cdot \boldsymbol{X})+$ $\left.+\boldsymbol{S}^{*}(\boldsymbol{L} \cdot \boldsymbol{X})\right) \subset \boldsymbol{S T}(\boldsymbol{K} \cdot \boldsymbol{X})+\boldsymbol{T}^{*} \boldsymbol{S}^{*}(\boldsymbol{L} \cdot \boldsymbol{X})$. Par conséquent:

$$
\boldsymbol{S S}^{*}(\boldsymbol{X}) \subset \boldsymbol{S T}(\boldsymbol{K} \cdot \boldsymbol{X})+\boldsymbol{T}^{*} \boldsymbol{S}^{*}(\boldsymbol{L} \cdot \boldsymbol{X}) \text {. }
$$

D'après le th. $43^{\text {b }}$ on a: $\boldsymbol{S T}(\boldsymbol{K} \cdot \boldsymbol{X})=\boldsymbol{T S}(\boldsymbol{K} \cdot \boldsymbol{X})$; en tenant compte de (3), on en conclut à l'aide du th. $51^{\circ}$ que $\boldsymbol{S} \boldsymbol{T}(\boldsymbol{K} \cdot \boldsymbol{X})=$ $=\boldsymbol{T} \boldsymbol{S}_{\beta}\left(\boldsymbol{K} \cdot \boldsymbol{X}\right.$ ). Comme $\boldsymbol{T} \boldsymbol{S}_{\beta} \in \mathfrak{M}$ (en vertu des th. $36^{\mathrm{a}}, 50^{\mathrm{a}}$ et $\mathrm{du}$ lem. $16^{\circ}$ ), il en résulte enfin que

$$
\boldsymbol{S T}(\boldsymbol{K} \cdot \boldsymbol{X}) \subset \boldsymbol{T} \boldsymbol{S}_{\beta}(\boldsymbol{K}) .
$$

D'une façon analogue on obtient:

$$
\boldsymbol{T}^{*} \boldsymbol{S}^{*}(L \cdot \boldsymbol{X}) \subset \boldsymbol{T}^{*} \boldsymbol{S}_{\beta}^{*}(\boldsymbol{L}),
$$

et les inclusions (4)-(6) entraînent immédiatement:

$$
\boldsymbol{S} \boldsymbol{S}^{*}(\boldsymbol{X}) \subset \boldsymbol{T} \boldsymbol{S}_{\beta}(\boldsymbol{K})+\boldsymbol{T}^{*} \boldsymbol{S}_{\beta}^{*}(\boldsymbol{L}) .
$$

Il est ainsi prouvé que toute classe $X$ qui remplit la formule (1) vérifie aussi linclusion (7). Par conséquent on a: $\sum \boldsymbol{S} \boldsymbol{S}^{*}(\boldsymbol{X}) \subset$ $C T S_{\beta}(\boldsymbol{K})+T^{*} S_{\beta}^{*}(\boldsymbol{L})$, d'où en raison de la déf. 21 :

$\boldsymbol{R}_{\beta}(\boldsymbol{K}+\boldsymbol{L}) \subset \boldsymbol{T} \boldsymbol{S}_{\beta}(\boldsymbol{K})+\boldsymbol{T}^{*} \boldsymbol{S}_{\beta}^{*}(\boldsymbol{L})$ pour toutes deux classes $\boldsymbol{K}$ et $\boldsymbol{L}$.

A l'aide des déf, $8^{b}$ et $9^{a}$ on en déduit sans peine un autre énoncé:

$\boldsymbol{R}_{\beta}[\boldsymbol{F}+\boldsymbol{G}] \stackrel{\circ}{\complement} \boldsymbol{T} \boldsymbol{S}_{\beta} \boldsymbol{F}+\boldsymbol{T}^{*} \boldsymbol{S}_{\beta}^{*} \boldsymbol{G}$ pour toutes deux opérations $\boldsymbol{F}$ et $\boldsymbol{G}$, ce qui achève la démonstration de ${ }^{2}$ ).
Passons enfin à g). Il résulte de la déf. 21 que

$$
\overline{\overline{\boldsymbol{R}_{\beta}(\cdot \overline{\boldsymbol{K}})}} \underset{\boldsymbol{X}_{\in} \boldsymbol{U}_{\beta}(\boldsymbol{K})}{\leqslant} \overline{\overline{S^{*}(\bar{X})}} .
$$

Suivant le th. $42^{8}$ et le lem. $19^{1}$ on a: $\overline{\overline{S S^{*}(\bar{X})}} \leqslant 2^{\overline{S^{*}(\bar{X})}} \leqslant$

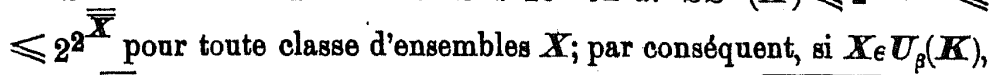
donc $\overline{\bar{X}}<\aleph_{\beta}$, on obtient à l'aide de la déf. $4: \overline{\overline{S S^{*}(X)}} \leqslant 2^{2 x_{\beta}}$. Comme de plus, d'après le lem. $10^{\circ}, \overline{\overline{U_{\beta}(\bar{K})}} \leqslant(\overline{\bar{K}})^{\alpha_{\beta}}$, on en conclut que

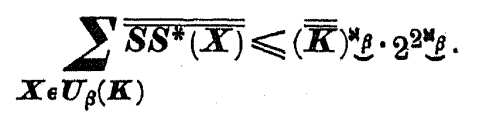

Les formules (8) et (9) entraînent immédiatement linégalité cherchée:

$$
\overline{\overline{\boldsymbol{R}_{\beta}(\overline{\boldsymbol{K}})}} \leqslant(\overline{\overline{\boldsymbol{K}}})^{\mathrm{k}_{\beta}} \cdot 2^{2^{\mathrm{w}_{\beta}} .}
$$

Le théorème en question est donc complètement démontré.

Théorème 56. ") Si $A \neq 0$ et $\beta=\sup (\bar{\varphi}(A))$, on a $\boldsymbol{R}_{\beta}=$ $=\sum_{x \in A}^{0} \boldsymbol{R}_{\varphi(x)}$

b) si $\beta \leqslant \gamma$, on a $\boldsymbol{R}_{\beta} \stackrel{\circ}{\complement} \boldsymbol{R}_{\gamma}$;

c) si $\gamma<\beta$, on a $\boldsymbol{R}_{\beta} \boldsymbol{R}_{\gamma}=\boldsymbol{R}_{\beta}$

d) si $\beta \leqslant c f(\gamma)$, on a $\boldsymbol{R}_{\beta} \boldsymbol{R}_{\gamma}=\boldsymbol{R}_{\gamma}$

o) si cf $(\beta)=\beta$, on a $\boldsymbol{R}_{\beta}^{2}=\boldsymbol{R}_{\beta}$;

) si $f(\beta)=\beta$, on $a\left[\boldsymbol{R}_{\beta}[I \stackrel{+}{ } \boldsymbol{C}]\right]^{2}=\boldsymbol{R}_{\beta}[I+C]$.

Démonstration des propositions a)- ${ }^{\circ}$ ) est complètement analogue à celle des parties correspondantes $\left.\left({ }^{\mathrm{a}}\right)-\mathrm{d}\right)$ et $\left.{ }^{\mathrm{f}}\right)$ ) du th. $\mathbf{5 2}$.

Quant à la formule ${ }^{\text {f), }}$, on raisonnera comme suit.

En appliquant le lem. $14^{\circ}$ et en tenant compte du lem. $17^{\mathrm{b}}$ et

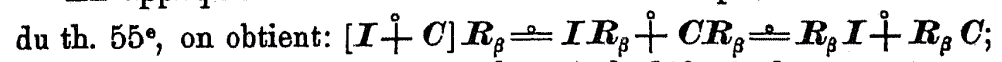
l'opération $\boldsymbol{R}_{\beta}$ étant monotone (th. $55^{\mathrm{a}}$ ), la déf. $12^{\mathrm{a}}$ donne en outre: $\boldsymbol{R}_{\beta} \boldsymbol{I} \subset \boldsymbol{R}_{\beta}[\boldsymbol{I}+\boldsymbol{C}]$ et $\boldsymbol{R}_{\beta} \boldsymbol{C} \check{C}^{\circ} \boldsymbol{R}_{\beta}[\boldsymbol{I} \dot{+} \boldsymbol{C}]$, d'où $\boldsymbol{R}_{\beta} \boldsymbol{I} \stackrel{+}{\boldsymbol{R}_{\beta}} \boldsymbol{C} \check{\complement}^{\circ}$ $\stackrel{\circ}{\complement} \boldsymbol{R}_{\beta}[\boldsymbol{I}+\boldsymbol{C}]$. Par conséquent,

$$
[\boldsymbol{I}+\boldsymbol{C}] \boldsymbol{R}_{\beta} \stackrel{\circ}{\complement} \boldsymbol{R}_{\beta}[\boldsymbol{I}+\boldsymbol{C}] \text {. }
$$

Fundamenta Mathematicae. T. XVI. 
Par une application renouvelée de la déf. $12^{a}$ on déduit de (1):

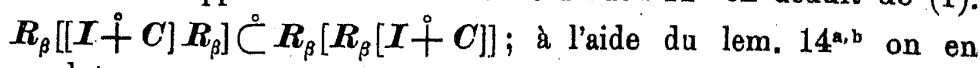
conclut que

(2) $\quad\left[\boldsymbol{R}_{\beta}[\boldsymbol{I}+\boldsymbol{C}]\right]\left[\boldsymbol{R}_{\beta}[\boldsymbol{I}+\boldsymbol{C}]\right] \stackrel{\circ}{\complement}\left[\boldsymbol{R}_{\beta} \boldsymbol{R}_{\beta}\right][[\boldsymbol{I}+\boldsymbol{C}][\boldsymbol{I} \dot{+} \boldsymbol{C}]]$.

Or, la déf. 10 permet d'écrire l'inclusion (2) sous la forme plus simple: $\left[\boldsymbol{R}_{\beta}[\boldsymbol{I}+\boldsymbol{C}]\right]^{2} \stackrel{\circ}{\complement}^{2} \boldsymbol{R}_{\beta}^{2}[\boldsymbol{I}+\boldsymbol{C}]^{2} ;$ comme $\boldsymbol{R}_{\beta}^{2} \rightleftharpoons \boldsymbol{R}_{\beta}$ (suivant la formule ${ }^{\text {e) }}$ du théorème en question) et $[\boldsymbol{I}+\boldsymbol{C}]^{2}=\boldsymbol{I}+\boldsymbol{C}$ (lem. 18c), il s'en suit que

$$
\left[\boldsymbol{R}_{\beta}[\boldsymbol{I} \stackrel{+}{+}]\right]^{2} \subset{ }^{\circ} \boldsymbol{R}_{\beta}[\boldsymbol{I}+\boldsymbol{C}] .
$$

D'autre part on a $\boldsymbol{I} \subset^{\circ} \boldsymbol{R}_{\beta}[\boldsymbol{I}+\boldsymbol{C}]$ (th. $55^{\mathrm{b}}$, lem. 17\%), d'où conformément au lem. $17^{\circ}$ :

(4) $\boldsymbol{R}_{\beta}[\boldsymbol{I} \stackrel{+}{C}] \stackrel{\circ}{\complement}\left[\boldsymbol{R}_{\beta}[\boldsymbol{I}+\boldsymbol{C}]\right]\left[\boldsymbol{R}_{\beta}[\boldsymbol{I}+\boldsymbol{C}]\right] \bumpeq\left[\boldsymbol{R}_{\beta}[\boldsymbol{I}+\boldsymbol{C}]\right]^{\mathrm{a}}$.

Les formules (3) et (4) entraînent tout de suite:

$$
\left[\boldsymbol{R}_{\beta}[\boldsymbol{I} \dot{+} \boldsymbol{C}]\right]^{2} \bumpeq \boldsymbol{R}_{\beta}[\boldsymbol{I}+\boldsymbol{C}] \text {, c. q. f. d. }
$$

Les th. $55^{a-c}$ el $56^{a-b}$ nous renseignent sur plusiears propriétés de l'opération $\boldsymbol{R}_{\beta}$, analogues à celles de $\boldsymbol{S}_{\beta}$ et $\boldsymbol{S}_{\beta}^{*}$. Notons encore quelques propriélés de ce.genre. On a les formules: $\boldsymbol{R}_{\beta}(0)=0, \boldsymbol{R}_{\beta}(\{\boldsymbol{A}\})=\{A\}$; $\boldsymbol{\Sigma} \boldsymbol{R}_{\beta}=\Sigma$ et $I I \boldsymbol{R}_{\beta}=\Pi_{;} \boldsymbol{M} \boldsymbol{R}_{\beta}=\boldsymbol{M}$. Par analogie au th. $\mathbf{5 1 ^ { \mathrm { d } } \text { , o on }}$; peut démontrér les théorémes: si $\overline{\overline{\boldsymbol{K}}}<\aleph_{\beta}$ ou $\overline{\overline{\Sigma(}(\overline{\boldsymbol{K}})}<\aleph_{\beta}$, on a $\boldsymbol{R}_{\beta}(\boldsymbol{K})=\boldsymbol{S \boldsymbol { S } ^ { * }}(\boldsymbol{K})$; si $\overline{\overline{1}}<\aleph_{\beta}$, on a $\boldsymbol{R}_{\beta}=\boldsymbol{S S}^{*}$. Par contre nous ne savons pas, si le th. $52^{a, s}$ se laisse également étendre sur l'opération $\boldsymbol{R}_{\beta}$.

Je passe à l'étude des classes closes par rapport aux opérations' $\dot{S_{\beta}}$ et $\boldsymbol{S}_{\beta}^{*}$. Il est à remarquer avant tout que cetté étude peut se borner aux nombres $\beta$ qui sont des indices des nombres initiaux réguliers (qui vérifient done la formule: $c f(\beta)=\beta$ ). Cela résulte, en. effet, du théorème suivant:

Théorème 57. Si $c f(\beta)<\beta$, on a $\mathscr{C}\left(\boldsymbol{S}_{\beta}\right)=\mathfrak{C}\left(\boldsymbol{S}_{\beta+1}\right)$ et $\mathscr{C}\left(\boldsymbol{S}_{\beta}^{*}\right)=$
$\mathcal{C}\left(\boldsymbol{S}_{*+1}^{*}\right)$.

Démonstration. En posant dans le th. 25: $F=S_{\beta}$ et $\nu=2$ et on tenant compte du th. $50^{\mathrm{b}}$, on obtient: $\mathcal{e} /\left(\boldsymbol{S}_{\beta}\right)=\mathcal{C} \mathcal{C}\left(\boldsymbol{S}_{\beta}^{2}\right)$, d'où suivant le th. 52x: $\mathscr{e}\left(\boldsymbol{S}_{\beta}\right)=\mathscr{Q} \mathscr{C}\left(\boldsymbol{S}_{\beta+1}\right)$; selon le cor. 27 on conclut que $\mathscr{e}\left(\boldsymbol{S}_{\beta}^{*}\right)=\mathscr{e}\left(\boldsymbol{S}_{\beta+1}^{*}\right)$, c. q. f. d.
A l'aide du cor. 22 et da th. $24^{\mathrm{a}}$ on pent tirer du théorème précédent quelques corollaires concernant les opérations de la forme $\boldsymbol{F}+\boldsymbol{S}_{\beta}$ et $\boldsymbol{F}^{+}+\boldsymbol{S}_{\beta}^{*}$ ( $\boldsymbol{F}$ étant une opération arbitraire), $\boldsymbol{F} \boldsymbol{S}_{\beta}$ et $\boldsymbol{F} \boldsymbol{S}_{\beta}^{*}$ (où $F$ est une opération nıonotone et adjonctive) etc. Je n'insiste pas sur ces questions, car elles n'interviendront pas dans la suite.

L'évaluation de la puissance d'une famille quelconque du type $\mathcal{C}^{A}\left(\boldsymbol{S}_{\beta}\right)$ ou $\mathcal{C}\left(\boldsymbol{S}_{\beta}^{*}\right)$ ne comporte pas de difficulté:

Théorème fondamental VIII. Si $\overline{\bar{I}}=\aleph_{\alpha}$, on a $\overline{\overline{\mathcal{Q C}\left(\overline{S_{\beta}}\right)}}=$ $\left.=\overline{\overline{\operatorname{CP}\left(S_{\beta}^{*}\right.}}\right)=2^{2^{*} \alpha}$

Démonstration, basée sur le th. $51^{\text {b }}$, est tout à fait analogue à celle du th. fond. IV.

Des difficultés beaucoup plus importantes apparaissent dans l'étude des familles telles que $\mathcal{C}\left(\boldsymbol{C}+\boldsymbol{S}_{\beta}\right), \mathcal{O}\left(\boldsymbol{T} \dot{+} \boldsymbol{S}_{\beta}\right), \mathscr{C}\left(\boldsymbol{S}_{\beta} \stackrel{\circ}{+} \boldsymbol{S}_{\gamma}^{*}\right)$. etc. Je vais commencer cette étude- par les lemmes suivants:

Lemme 58. Si $\overline{\bar{I}}=\kappa_{\alpha}$, il existe une classe d'ensembles $\boldsymbol{K}$ vérifiant

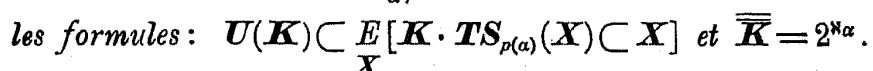

Démonstration. Selon le lem. 40 ilrexiste une classe d'ensembles $\boldsymbol{L}$ telle que

$$
\boldsymbol{U}(\boldsymbol{L}) \subset_{\boldsymbol{X}}^{E}[\boldsymbol{L} \cdot \boldsymbol{T}(\boldsymbol{X}) \subset \boldsymbol{X}] \text { et } \overline{\overline{\boldsymbol{L}}}=2^{\mathrm{s} \alpha} .
$$

Nous examinerons ici la famille $\overline{\boldsymbol{U}_{p(\alpha)}}(\boldsymbol{L})$. Remarquons d'abord que la déf. $5^{\text {b }}$ eniraîne la formule: $\boldsymbol{\Sigma} \boldsymbol{U}_{\beta}(X)=X$ pour tout ensemble $X$ et tout nombre $\beta$. Par conséquent, quels que soient $X$ et $Y$, si $U_{\beta}(X)=U_{\beta}(Y)$, on a $\Sigma U_{\beta}(X)=\Sigma U_{\beta}(Y)$, d'où $X=Y$; la fónction $U_{\beta}$ est donc biunivoque. Il en résulte en particulier (pour $\beta=p(\alpha)$ ) que toute classế d'ensembles $\boldsymbol{X}$ est de la puissance égale à celle de son image $\overline{\boldsymbol{U}_{p(\alpha)}}(\boldsymbol{X})$ :

$$
\overline{\overline{\boldsymbol{X}}}=\overline{\overline{\overline{\boldsymbol{U}_{p(a)}}(\boldsymbol{X})}} \text { pour t te classe } \boldsymbol{X},
$$

d'où selon (1)

$$
\overline{\overline{\overline{U_{p(\alpha)}}(\boldsymbol{L})}}=2^{* \alpha} .
$$


Nous allons montrer à présent que la famille $\overline{U_{p(\alpha)}}(\boldsymbol{L})$ vérifie la formule

$$
\boldsymbol{U} \overline{\boldsymbol{U}_{p(\alpha)}}(L) \subset \underset{\mathfrak{Q}}{E}\left[\overline{\boldsymbol{U}_{p(\alpha)}}(\boldsymbol{L}) \cdot \boldsymbol{T} \boldsymbol{S}_{p(\alpha)}(\mathfrak{Q}) \subset \mathfrak{e}\right]
$$

Supposons, contrairement à (4), qu'il existe une famille de classes $2 \mathcal{e}$ telle que

(5) $\quad \mathfrak{e} \subset \overline{\boldsymbol{U}_{p(\alpha)}}(\boldsymbol{L})$ el $\overline{\boldsymbol{U}_{p(\alpha)}}(\boldsymbol{L}) \cdot \boldsymbol{T} \boldsymbol{S}_{p(\alpha)}(\mathfrak{Q})-\mathfrak{d} \neq 0$.

(5) implique l'existence d'une classe $\boldsymbol{M}$ satisfaisant aux conditions:

$$
\begin{array}{ll}
(6) & \boldsymbol{M} \in \overline{U_{p(\alpha)}}(\boldsymbol{L})-\mathfrak{e} \\
\text { et } & \boldsymbol{M}_{\boldsymbol{E}} \boldsymbol{T} \boldsymbol{S}_{p(\alpha)}(\mathfrak{Q}) .
\end{array}
$$

D'après lá déf. 17 (pour $\boldsymbol{K}=\boldsymbol{S}_{p(\alpha)}$ (囚)), on conclut de (7) qu'il existe une classe $\boldsymbol{M}_{1}$ telle que $\boldsymbol{M} \subset \boldsymbol{M}_{1} \in \boldsymbol{S}_{p(\alpha)}(\mathfrak{Q C})$. En remplaçant dans la déf. $20 \boldsymbol{K}$ par ee et $\beta$ par $p(\boldsymbol{\alpha})$, on en déduit l'existence d'une famille $\mathfrak{Q}_{1}$ vérifiant les formules: $\boldsymbol{M}_{1}=\boldsymbol{\Sigma}\left(\mathfrak{C}_{1}\right)$ et $\mathfrak{C}_{1} \in \boldsymbol{U}_{p(\alpha)}(\mathfrak{Q} \mathfrak{Q})$. On a donc finalement, en raison de la déf. $5^{\mathrm{b}}$,

$$
\begin{gathered}
M \subset \Sigma\left(\mathfrak{Q}_{1}\right), \\
\mathfrak{e}_{1} \subset \mathfrak{e} \text { et } \overline{\overline{\mathfrak{Q}_{1}}}<\aleph_{p(\alpha)} .
\end{gathered}
$$

Les formules (5) et (9) donnent: $\mathfrak{C}_{1} \subset \overline{U_{p(\alpha)}}(\boldsymbol{L})$. A l'aide du theorème connu sur les images des ensembles (si $A \subset \bar{f}(B)$, il existe un ensemble $B_{1}$ tel que $A=\bar{f}\left(B_{1}\right)$ et $B_{1} \subset B$ ), on en conclut qu'il existe une classe $L_{1}$ remplissant les conditions:

$$
\mathfrak{e}_{1}=\overline{U_{p(a)}}\left(\boldsymbol{L}_{1}\right) \text { et } \quad \boldsymbol{L}_{1} \subset \boldsymbol{L} .
$$

Il résulte de (2) el (10) que $\overline{\overline{\text { ¿ }_{1}}}=\overline{\overline{\boldsymbol{L}}_{1}}$, d'où selon (9)

$$
\overline{\overline{L_{1}}}<\aleph_{p(\alpha)} \text {. }
$$

En vertu de (6) et (9), $\boldsymbol{M}_{\epsilon} \overline{\boldsymbol{U}_{p(\alpha)}}\left(\boldsymbol{L}_{1}\right)-\alpha_{1}$, done, en raison de (10), $\boldsymbol{M} \bar{\epsilon} \overline{U_{p(\alpha)}}(\boldsymbol{L})-\overline{\boldsymbol{U}_{p(\alpha)}}\left(\boldsymbol{L}_{1}\right)$. En appliquant la formule connue concernant la différence des images $(\bar{f}(A)-\bar{f}(B) \subset \bar{f}(A-B))$, or en obtient ensuite: $\boldsymbol{M}_{\epsilon} \overline{U_{p(\alpha)}}\left(L-\boldsymbol{L}_{1}\right)$; par conséquent, suivant la déf. 6 , il existe un ensemble $A$ tel que et

$$
\boldsymbol{M}=\boldsymbol{U}_{p(\alpha)}(A)
$$$$
A \in \boldsymbol{L}-\boldsymbol{L}_{1} .
$$

Selon (1) et (10) $\boldsymbol{L} \cdot \boldsymbol{T}\left(\boldsymbol{L}_{1}\right) \subset \boldsymbol{L}_{1}$; on en obtient à fortiori en vertu de (13): $\{A\} \cdot \boldsymbol{T}\left(\boldsymbol{L}_{1}\right) \subset \boldsymbol{L}_{1}$. $\left(\boldsymbol{L}-\boldsymbol{L}_{1}\right)=0$, d'où $A \bar{\epsilon} \boldsymbol{T}\left(\boldsymbol{L}_{1}\right)$. A l'aide de la déf. 17 on en conclut que $A \bar{\epsilon} U(Z)$, done que $A-Z \neq 0$ pour tout ensemble $Z$ de la classe $\boldsymbol{L}_{1}$. En appliquant l'axiome du choix, on peut done faire correspondre à tout ensemble $Z$ de $\boldsymbol{L}_{1}$ un élément $f(Z)$ de façon que l'on ait

$$
f(Z) \in A-Z \text { pour } Z \in \boldsymbol{L}_{1} .
$$

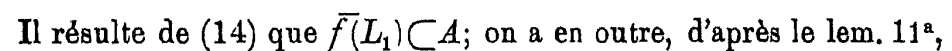
$\overline{\overline{f\left(\boldsymbol{L}_{1}\right)}} \leqslant \overline{\boldsymbol{\boldsymbol { L }}_{1}}$, d'où, en raison de $(11), \overline{\overline{\bar{f}\left(\boldsymbol{L}_{1}\right)}}<\aleph_{p(\alpha)}$. On en obtient aussitôt: $\bar{f}\left(\boldsymbol{L}_{1}\right) \in \boldsymbol{U}_{p(\alpha)}(A)$, ce qui, rapproché de (12), donne :

$$
\bar{f}\left(\boldsymbol{L}_{1}\right) \in \boldsymbol{M} \text {. }
$$

On conclut ensuite de (14) que $\bar{f}\left(\boldsymbol{L}_{1}\right)-Z \neq 0$ pour tout ensemble $Z$ de $\boldsymbol{L}_{1}$, donc que $\bar{f}\left(\boldsymbol{L}_{1}\right) \bar{\epsilon} \boldsymbol{U}_{p(\boldsymbol{\alpha})}(Z)$. Par conséquent, l'ensemble $\bar{f}\left(\boldsymbol{L}_{1}\right)$ n'appartient à aucune des classes formant la famille $\overline{\boldsymbol{U}_{p(a)}}\left(\boldsymbol{L}_{1}\right)$, d'où en vertu de (10)

$$
\bar{f}\left(\boldsymbol{L}_{1}\right) \bar{\epsilon} \boldsymbol{\Sigma}\left(\mathfrak{Q}_{1}\right) .
$$

(8) et (16) donnent tout de suite:

$$
\bar{f}\left(\boldsymbol{L}_{1}\right) \overline{\boldsymbol{\epsilon}} \boldsymbol{M} .
$$

Or, les formules (15) et (17) étant contradictoires, la supposition (5) est fausse, et la famille $\overline{U_{p(\alpha)}}(\boldsymbol{L})$ vérifie en conséquence la formule (4).

Observoins encore que pour $X_{\epsilon} L$ on a évidemment $X \subset 1$, d'où $\boldsymbol{U}_{p(\alpha)}(X) \subset \boldsymbol{U}_{p(\alpha)}(1)$ et $\boldsymbol{U}_{p(\alpha)}(X) \in \boldsymbol{U} \boldsymbol{U}_{p(\alpha)}(I)$; il en résulte que

$$
\overline{\boldsymbol{U}_{p(\alpha)}}(\boldsymbol{L}) \subset \boldsymbol{U} \boldsymbol{U}_{p(\alpha)}(1) \text {. }
$$

Si l'on remplace enfin dans le lem. $10^{\alpha} A$ par 1 et $\beta$ par $p(\alpha)$, on obtient par hypothèse:

$$
\overline{\overline{U_{p(\alpha)}(1)}}=\aleph_{\alpha}=1 .
$$


Posons momentanément: $1^{*}=U_{p(\alpha)}(1)$ et $\boldsymbol{K}^{*}=\overline{U_{p(\alpha)}}(\boldsymbol{L})$. Les formules (3), (4) et (18) montrent que pour l'ensemble $1^{*}$ on peut construire une classe de ses sous-ensembles $\boldsymbol{K}^{*}$ qui vérifie la thèse du lemme en question. Il est évident que l'existence d'une telle classe de sous-ensembles constitue une propriété de l'ensernble invariante par rapport à toutes les transformations biunivoques; par conséquent, lorsque cette propriété se présente pour un ensemble, elle se présente nécessairement pour tout ensemble de la même puissance. Or, en vertu de (19), les ensembles $1^{*}$ et 1 ont les puissances égales; on peut donc construire dans l'ensemble 1 une classe d'ensembles $\boldsymbol{K}$ remplissant les deux conditions de la thèse, c. $q$ f. d.

Dans cette démonstration nous étions contraints d'élargir le domaine primitif des opérations $\boldsymbol{T}$ et $\boldsymbol{S}_{\boldsymbol{\beta}}$ et de les appliquer non aux classes d'ensembles, mais anx familles de telles classes; nons avons établi ainsi nne propriété des ensembles de rang inférieur par intermédiaire des ensembles de rang supérieur. On applique fréquemment une pareille méthode de raisonnement dans les diverses démonstrations da domaine de la Théorie générale des Ensembles; cependant, aussi bien dans notre cas particulier que dans d'autres cas, la question, si cette méthode du passage par les ensembles de rang supérieur est inévitable, reste encore ouverte.

Lemme 59. Si la classe d'ensembles $\boldsymbol{K}$ vérifie les formules: $\boldsymbol{U}(\boldsymbol{K}) \subset \underset{\boldsymbol{X}}{E}\left[\boldsymbol{K} \cdot \boldsymbol{T} \boldsymbol{S}_{\beta}(\boldsymbol{X}) \subset \boldsymbol{X}\right]$ et $\overline{\boldsymbol{K}} \geqslant \aleph_{\beta}$, on a aussi:

a) $\boldsymbol{U}(\boldsymbol{K}) \subset \underset{X}{E}\left[\boldsymbol{K} \cdot \boldsymbol{C T S}_{\beta}(\boldsymbol{X}) \subset \boldsymbol{X}\right]$;

b) $\boldsymbol{U}(\boldsymbol{K}) \subset_{\boldsymbol{X}}^{E}\left[\boldsymbol{K} \cdot \boldsymbol{R}_{\beta}\left[\boldsymbol{I}^{\circ} \boldsymbol{C}\right](\boldsymbol{X}) \subset \boldsymbol{X}\right]$.

Démonstration. a) Supposons, contrairement à la thése du lemme, que

$$
\boldsymbol{U}(\boldsymbol{K})-\underset{\boldsymbol{X}}{E}\left[\boldsymbol{K} \cdot \boldsymbol{C T S}_{\beta}(\boldsymbol{X}) \subset \boldsymbol{X}\right] \neq 0
$$

ils existent donc une classe d'ensemble $L$ et un ensemble $A$ qui satisfont aux conditions:

$$
\boldsymbol{L} \subset \boldsymbol{K}, A \in \boldsymbol{K}
$$

$$
A \in C \boldsymbol{T} \boldsymbol{S}_{\beta}(\boldsymbol{L}) \text {. }
$$

En appliquant successivement la déf. 14 à la classe $\boldsymbol{K}=\boldsymbol{T S} \boldsymbol{S}_{\beta}(\boldsymbol{L})$, la déf. 17 à $\boldsymbol{K}=\boldsymbol{S}_{\beta}(\boldsymbol{L})$ et la déf. 20 à $\boldsymbol{K}=\boldsymbol{L}$, on déduit de (3) l'existence d'une classe $M$ qui vérifie les formules:

La formule (4) entraîne:

$$
\overline{\overline{M+\{A}\}}<\aleph_{\beta} ;
$$

or, $\boldsymbol{K}$ étant par hypothèse de puissance $\geqslant \aleph_{\beta}$, on en conclut que

$$
\boldsymbol{K}-(\boldsymbol{M}+\{A\}) \neq 0 .
$$

En rapprochant d'autre part (2) et (6), on obtient: $M+\{A\} \subset \boldsymbol{K}$; d'après l'hypothèse du lemme il en résulte que

$$
\boldsymbol{K} \cdot \boldsymbol{T S} \boldsymbol{S}_{\beta}(\boldsymbol{M}+\{A\}) \subset \boldsymbol{M}+\{A\} .
$$

L'inclusion (6) donne notoirement: $\boldsymbol{\Sigma}(\boldsymbol{M}+\{A\})=\boldsymbol{\Sigma}(\boldsymbol{M})+$ $+A=1$. En raison du th. $43^{a}$ il s'en suit que $\boldsymbol{T S}(\boldsymbol{M}+\{A\})=$ $=\boldsymbol{U}(1)$; comme en outre $\boldsymbol{S}_{\beta}(\boldsymbol{M}+\{A\})=\boldsymbol{S}(\boldsymbol{M}+\{A\})$ (suivant le th. $51^{\circ}$ et la formule (7)), on a finalement:

$$
\boldsymbol{T S} \boldsymbol{S}_{\beta}(\boldsymbol{M}+\{\boldsymbol{A}\})=\boldsymbol{V}(1) .
$$

Or, la formule (10) veut dire que la classe $\boldsymbol{T S} \boldsymbol{S}_{\beta}(\boldsymbol{M}+\{A\})$ se compose de tous les ensembles possibles (contenus dans 1). "Ona a par conséquent $\boldsymbol{K} \subset \boldsymbol{T} \boldsymbol{S}_{\beta}(\boldsymbol{M}+\{A\})$, d'où suivant (9):

$$
\boldsymbol{K} \subset \boldsymbol{M}+\{\boldsymbol{A}\}
$$

La contradiction évidente entre les formules (8) et (10) réfuse la supposition (1); nous sommes donc contraints d'admettre que

$$
\boldsymbol{U}(\boldsymbol{K}) \subset \underset{\boldsymbol{X}}{\boldsymbol{E}}\left[\boldsymbol{K} \cdot \boldsymbol{C T S _ { \beta }}(\boldsymbol{X}) \subset \boldsymbol{X}\right] \text {, c. q. f. d. }
$$

b) Conformément aux déf. 9a et 13 et en vertu du th. $55^{t}$, on a pour toute classe d'ensembles $\boldsymbol{X}: \boldsymbol{R}_{\beta}[\boldsymbol{I}+\boldsymbol{C}](\boldsymbol{X})=\boldsymbol{R}_{\beta}(\boldsymbol{X}+\boldsymbol{C}(\boldsymbol{X})) \subset$ 
$C \boldsymbol{T} \boldsymbol{S}_{\beta}(\boldsymbol{X})+\boldsymbol{T}^{*} \boldsymbol{S}_{\beta}^{*} \boldsymbol{C}(\boldsymbol{X})$, d'où, en raison du lem.19^, $\boldsymbol{R}_{\beta}[\boldsymbol{I}+\boldsymbol{C}](\boldsymbol{X}) \subset$ $\mathcal{C T S _ { \beta } ( X )}+\boldsymbol{C T S _ { \beta }}(X)$. Il s'en suit aussitôt que

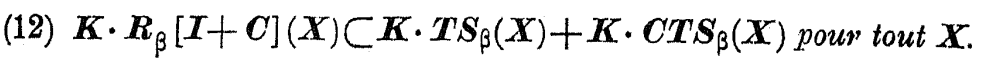

Or, soit $\boldsymbol{X} \subset \boldsymbol{K}$; l'hypothèse du lemme et la formule a) qui vient d'être établie impliquent alors: $\boldsymbol{K} \cdot \boldsymbol{T S} \boldsymbol{S}_{\beta}(\boldsymbol{X}) \subset \boldsymbol{X}$ et $\boldsymbol{K} \cdot \boldsymbol{C T} \boldsymbol{S}_{\beta}(\boldsymbol{X}) \subset \boldsymbol{X}$; en le rapprochant de (12), nous en concluons que $\boldsymbol{K} \cdot \boldsymbol{R}_{\beta}[\boldsymbol{I}+\boldsymbol{C}](\boldsymbol{X}) \subset \boldsymbol{X}$. Par conséquent,

$$
\boldsymbol{U}(\boldsymbol{K}) \subset_{\boldsymbol{X}}^{E}\left[\boldsymbol{K} \cdot \boldsymbol{R}_{\beta}[\boldsymbol{I}+\boldsymbol{C}](\boldsymbol{X}) \subset \boldsymbol{X}\right] \text {, c. q. f. d. }
$$

Sans changer la démonstration précédente, on peut remplacer dans le lem. 59 la formule a) par la formule plus forte qui suit: $\boldsymbol{U}(\boldsymbol{K})$ $\mathcal{C} \underset{\boldsymbol{X}}{\boldsymbol{E}}\left[\boldsymbol{K} \cdot \boldsymbol{C} \boldsymbol{T} \boldsymbol{S}_{\beta}(\boldsymbol{X})=0\right]$ Une remarque analogue s'applique an lem. $3 \overline{5}$ du $\S 3$.

Corollaire 60. Si $\overline{\bar{I}}=\aleph_{a}$, il existe une classe d'ensembles $\boldsymbol{K}$ vérifiant les formules: $\boldsymbol{U}(\boldsymbol{K}) \subset \underset{\boldsymbol{X}}{\boldsymbol{E}}\left[\boldsymbol{K} \cdot \boldsymbol{R}_{p r \alpha)}[\boldsymbol{I}+\boldsymbol{C}](\boldsymbol{X}) \subset \boldsymbol{X}\right]$ et $\boldsymbol{K}=2^{\mathrm{N} a}$.

Démonstration. Conformément au lem. 58, il existe une

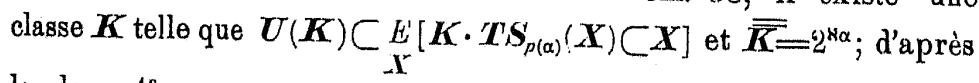
les lem. $4^{\mathrm{a}}$

et $1^{\mathrm{a}}$ on a évidemment $\overline{\bar{K}}>\boldsymbol{N}_{\alpha} \geqslant \boldsymbol{s}_{p(\alpha)}$. En posant donc dans le lem. $59^{\mathrm{b}}: \beta=p(\alpha)$, on se convaint immédiatement que $\boldsymbol{K}$ est la classe cherchée.

Théorèmé 61. Si $\overline{\bar{I}}=\boldsymbol{\aleph}_{\alpha}$ et $\beta \leqslant p(\alpha)$, on a $\overline{\overline{\mathcal{Q P}\left(\boldsymbol{C}+\boldsymbol{R}_{\beta}\right)}}=2^{2 \mathrm{2}^{* \alpha}}$.

Démonstration. En teuant compte que $c f(p(\alpha))=p(\alpha)$ d'après le lem. $4^{b}$, on conclut du th. $56^{t}$ (pour $\beta=p(\alpha)$ ) que l'opération

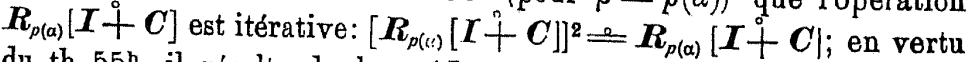
du th. $55^{\text {b }}$, il résultẹ du lem. $17^{\circ}$ que cette opération est de plus adjonctive: $\boldsymbol{I} \stackrel{\circ}{C}^{R_{p(\alpha)}}[I+C]$. Par conséquent, nous pouvons appliquer le lem. $34^{\mathrm{b}}$, en y posant: $\boldsymbol{F}=\boldsymbol{R}_{p(a)}[\boldsymbol{I}+\boldsymbol{C}]$; en rapprochant ce lemme du cor. 60 , nous obtencns:

$$
\overline{\overline{\mathcal{Q}\left(\boldsymbol{R}_{p(\alpha)}[\boldsymbol{I}+\boldsymbol{C}]\right)}}=2^{2^{\mathrm{N} \alpha}} \text {. }
$$

Comme $\boldsymbol{R}_{p(\alpha)} \in \mathfrak{M}, \boldsymbol{I C}^{\circ} \boldsymbol{R}_{p(\alpha)}$ (th. $\left.55^{\mathrm{a}, \mathrm{b}}\right)$ et $\boldsymbol{I} C^{\circ} \boldsymbol{I}+\boldsymbol{C}$, le th $24^{\mathrm{d}}$ pour $\boldsymbol{F} \rightleftharpoons \boldsymbol{R}_{p(a)}$ et $\boldsymbol{G} \rightleftharpoons \boldsymbol{I}+\boldsymbol{C}$ donne: $\mathscr{e}\left(\boldsymbol{R}_{p(\alpha)}[\boldsymbol{I}+\boldsymbol{C}]\right)=$
$=\operatorname{err}\left(\boldsymbol{R}_{p(\alpha)} \dot{\dagger} \dot{\boldsymbol{C}} \boldsymbol{C}\right)$, d'où suivant (1):

$$
\overline{\overline{\mathcal{Q} P\left(\boldsymbol{R}_{p(\alpha)}+\boldsymbol{I}+\boldsymbol{C}\right)}}=2^{\tilde{2}^{\mathrm{N}_{\alpha}} .}
$$

En raison du th. $56^{\text {b }}$, linégalité: $\beta \leqslant p(\alpha)$, donnée par l'hypothèse, entraîne: $\boldsymbol{R}_{\beta} \check{\circ}^{\circ} \boldsymbol{R}_{p(\alpha)}$, donc $\boldsymbol{C} \stackrel{\circ}{+} \boldsymbol{R}_{\beta} \subset \boldsymbol{R}_{p(\alpha)}+\boldsymbol{T}+\boldsymbol{C}$. A l'aide du th. $21^{a}$ on en conclut que

$$
\mathscr{e}\left(\boldsymbol{R}_{p(\alpha)}+\boldsymbol{I}+\boldsymbol{C}\right) \subset \mathcal{Q} \mathcal{C}\left(\boldsymbol{+} \boldsymbol{R}_{\beta}\right) .
$$

Les formules (2) et (3) impliquent aussitôt que $\overline{\overline{\mathcal{C P}\left(\boldsymbol{C}+\overline{+} \boldsymbol{R}_{\beta}\right)}} \geqslant$ $\geqslant 2^{2^{2 \alpha_{\alpha}}} ;$ l'inégalité inverse étant également remplie d'après le th. 32 , on a donc finalement:

$$
\overline{\overline{\mathcal{C} P\left(\boldsymbol{C}+\boldsymbol{R}_{\beta}\right)}}=2^{2^{* \alpha} \alpha}, \quad \text { c. q. f. d. }
$$

Corollaire 62. Si $\overline{\bar{I}}=\aleph_{\alpha}, \beta \leqslant p(\alpha) \quad \boldsymbol{F}^{\prime} \in \mathfrak{H}_{\beta}$ et si en outre

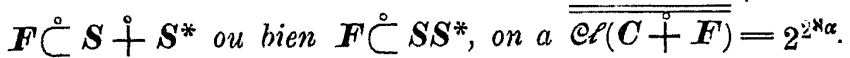

Démonstration. A l'aide das lem. $17^{c}$ et $19^{1}$ on déduit sans peine du th. $42^{a, b}$ les inclusions: $\boldsymbol{S}^{\circ} \boldsymbol{S S}^{*}$ et $\boldsymbol{S}^{*} \mathcal{C}^{\circ} \boldsymbol{S} \boldsymbol{S}^{*}$, donc également: $\boldsymbol{S}+\boldsymbol{S}^{*} \stackrel{\complement}{\complement} \boldsymbol{S} \boldsymbol{S}^{*}$. Par conséquent, la formule: $\boldsymbol{F} \complement^{\circ} \boldsymbol{S}+\boldsymbol{S}^{*}$ implique: $\boldsymbol{F}^{\circ} \boldsymbol{S S}^{*}$, de sorte qu'il est superflu d'examiner la première de ces inclusions séparément.

Or, conformément au th. $55^{\mathrm{c}}$, les formules: $\boldsymbol{F} \in \mathfrak{Z}_{\beta}$ et $\boldsymbol{F} \complement^{0} \boldsymbol{S} \boldsymbol{S}^{*}$ donnent: $\boldsymbol{F} \stackrel{\circ}{\complement} \boldsymbol{R}_{\beta}$, dunce aussi: $\boldsymbol{C}+\boldsymbol{F} \check{C} \boldsymbol{C}+\boldsymbol{R}_{\beta}$, d'où en vertu du th. $21^{\text {a }} \mathscr{e}\left(\boldsymbol{C}+\boldsymbol{R}_{\beta}\right) \subset \mathscr{e}(\boldsymbol{C}+\boldsymbol{F})$; comme d'après le th. 61 $\overline{\overline{\mathcal{Q}\left(\boldsymbol{C}+\overline{+} \overline{\left.\boldsymbol{R}_{\beta}\right)}\right.}}=2^{2^{\mathrm{N} \alpha}}$ et en raison du th. $32 \overline{\overline{\mathcal{Q} \ell(\boldsymbol{C}+\boldsymbol{F})}} \leqslant 2^{2^{\mathrm{N} \alpha}}$, on en obtient tout de suite:

$$
\overline{\overline{\mathcal{Q} P(C+F)}}=2^{2^{* \alpha},}, \text { c. q. f. d. }
$$

Theorème fondamental IX. Si $\overline{\bar{I}}=\aleph_{\alpha}$ et $\beta \leqslant p(a)$, on $a$ $\overline{\overline{\mathcal{C}\left(\boldsymbol{C}+\boldsymbol{S}_{\beta}\right)}}=2^{2^{\alpha \alpha}}$.

Démonstration. En vertu des th. $50^{\text {a }}$ et $51^{\text {b }}$, ce théorème n'est qu'un cas particulier du corollaire précédent.

Théorème fondamental X. Si $\overline{1}={ }_{\aleph_{\alpha}}$ et $\beta \leqslant p(a)$, on a $\overline{\overline{\overline{C P}\left(\boldsymbol{T}+\boldsymbol{S}_{\beta}\right)}}=$ $\left.=\overline{\overline{\mathcal{C} P\left(\boldsymbol{T}^{*}+\boldsymbol{S}_{\beta}^{*}\right.}}\right)=2^{2^{\boldsymbol{N}_{\alpha}}}$. 
Démonstration. D'après le th. $36^{\mathrm{b}}$ l'opération $\boldsymbol{T}$ est itérative $\left(\boldsymbol{T}^{2}=\boldsymbol{T}\right.$ ); en vertu du lem. $4^{\mathrm{b}}$, le th. $52^{\mathrm{t}}$ (pour $\beta=p(\alpha)$ ) prouve que l'opération $\boldsymbol{S}_{p(\alpha)}$ l'est aussi. Comme de plus $\boldsymbol{T} \boldsymbol{S}_{p(\alpha)} \rightleftharpoons \boldsymbol{S}_{p(\alpha)} \boldsymbol{T}$

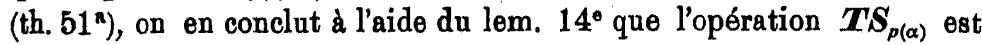
également itérative. Suivant le lem. $17^{\circ}$ et les th. $36^{\mathrm{b}}$ et $50^{\mathrm{b}}$ l'opération $^{\prime}$ $\boldsymbol{T} \boldsymbol{S}_{p(\alpha)}$ est en outre adjonctive.

Ces faits établis, on raisonne tout comme dana la démonstration du th. 61 , à cette différence près qu'au lieu du cor. 60 on fait usage du lem. 58. On parvient ainsi à l'égalité: $\overline{\overline{\mathcal{E P}\left(T+S_{\beta}\right)}}=2^{2^{N_{\alpha}}}$. Or, il résulte du th. 33 et du lem. $19^{\mathrm{d}}$ que $\overline{\overline{\mathcal{Q} P\left(T^{*}+S_{\beta}^{*}\right)}}=$ $=\overline{\overline{\mathcal{Q}\left(\left[\boldsymbol{T}+\boldsymbol{S}_{\beta}\right]^{*}\right)}}=\overline{\overline{\mathcal{Q} \mathcal{Q}\left(\boldsymbol{T}+\boldsymbol{S}_{\beta}\right)}}$. En rapprochant les deux formules obtenues, on voit done que

$$
\overline{\overline{\mathcal{Q}\left(T+\boldsymbol{T}_{\beta}\right)}}=\overline{\overline{\mathcal{Q} \mathcal{C}\left(\boldsymbol{T}^{*}+\boldsymbol{S}_{\beta}^{*}\right)}}=2^{2^{\mathrm{N} \alpha}} \text {, c. q. f. d. }
$$

Corollaire 63: $\left.{ }^{a}\right) S i \overline{\bar{I}}=\aleph_{\alpha}$ et $\beta \leqslant p(\alpha)$, chacune des formules:

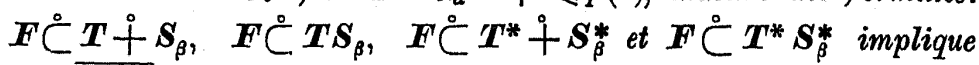
que $\overline{\overline{\mathcal{Q}(\bar{F})}}=2^{2^{* \alpha} \alpha}$;

b) si $\overline{\overline{1}}=\aleph_{\alpha}, \beta \leqslant p(\alpha)$ et $\boldsymbol{F} \in \mathfrak{X}_{\beta}$, chacune des formules: $\boldsymbol{F} \subset$ $\dot{\complement} \boldsymbol{T}+\boldsymbol{S}, \boldsymbol{F} \subset^{\circ} \boldsymbol{T S}, \boldsymbol{F}^{\circ} \boldsymbol{T}^{*}+\boldsymbol{S}^{*}$ et $\boldsymbol{F} \subset^{\circ} \boldsymbol{T}^{*} \boldsymbol{S}^{*}$ implique que $\overline{\overline{C P}(\bar{F})}=2^{2 \times \alpha}$.

Démonstration est analogue à celle du th. 49 et du cor. 62 ; pour déduire b) de a) on fait usage du th. 51.

Dans le cor. $63^{b}$ nous avons réussi d'évaluer la puissance des familles $\mathcal{C}(\boldsymbol{F})$ pour une classe assez vaste d'opérations $\boldsymbol{F}$, à savoir pour toutes les opérations qui sont à la fois semi-additives au dégré $\beta \leqslant p(\alpha)$ (où $\overline{\overline{1}}=\aleph_{a}$ ) et intrinsèques.

Théorème fondamentale XI. Si $\overline{\bar{I}}=\aleph_{\alpha}$ et $\beta \leqslant p(a)$, on $a$

a) $\left.\overline{\overline{\mathcal{C}\left(S+S_{\beta}^{*}\right.}}\right)=\overline{\overline{\mathcal{Q} \ell\left(S^{*}+S_{\beta}\right)}}=2^{2^{2_{\alpha} \alpha}}$,

b) $\overline{\overline{\mathcal{C}\left(S_{\beta}+S_{\gamma}^{*}\right)}}=\overline{\overline{\mathcal{Q}\left(S_{\gamma}+S_{\beta}^{*}\right)}}=2^{2^{n_{\alpha}}}$.

Démonstration. En raison du th. $51^{\mathrm{b}}$ (pour $\beta=\gamma$ ) et de la formule: $S_{\gamma}^{*} \complement^{\circ} S^{*} \complement^{\circ} T$, qui en résulte d'après le lem. $19^{\mathrm{b}, \mathrm{o}}$, on a les inclusions suivantes: $\boldsymbol{S}+\boldsymbol{S}_{\beta}^{*} \mathcal{C}^{\circ} \boldsymbol{T}^{*}+\boldsymbol{S}_{\beta}^{*}, \boldsymbol{S}^{*}+\boldsymbol{S}_{\beta} \mathcal{C} \boldsymbol{T}+\boldsymbol{S}_{\beta}$,

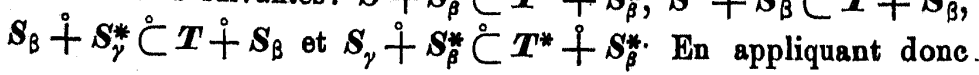

le cor. 59a (pour $\boldsymbol{F} \bumpeq \boldsymbol{S}+\boldsymbol{S}_{\beta}^{*}, \boldsymbol{F}=\boldsymbol{S}^{*} \stackrel{+}{+} \boldsymbol{S}_{\beta}$ etc.), on parvient aussitôt aux formules cherchées.

Comme une autre conséquence du cor. $63^{\mathrm{a}}$ citons le suivant

Théorème 64. Si $\overline{\overline{1}}=\aleph_{\alpha}$ et $\beta \leqslant p(a)$, on a $\overline{\overline{\mathcal{C}_{i}\left(\boldsymbol{R}_{\beta}\right)}}=$

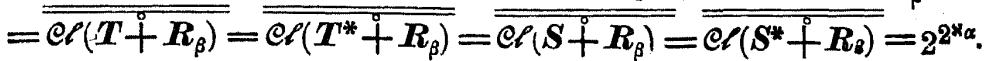

Démonstration. A l'aide des th. $36^{\mathrm{a}}, 50^{\mathrm{a}}, 51^{\mathrm{b}}, 55^{\mathrm{d}}$ et des

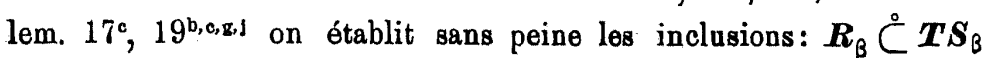
et $\boldsymbol{R}_{\beta} \stackrel{\circ}{\complement} \boldsymbol{T}^{*} \boldsymbol{S}_{\beta}^{*}, \boldsymbol{T} \stackrel{\circ}{+} \boldsymbol{R}_{\beta} \stackrel{\circ}{\complement} \boldsymbol{T} \boldsymbol{S}_{\beta}, \boldsymbol{T}^{*} \stackrel{\circ}{+} \boldsymbol{R}_{\beta} \stackrel{\circ}{\complement} \boldsymbol{T}^{*} \boldsymbol{S}_{\beta}^{*}, \boldsymbol{S}+\boldsymbol{R}_{\beta} \stackrel{\circ}{\complement}$ $\dot{\complement} T^{*} S_{\beta}^{*}$ et $S^{*}+R_{\beta} \check{C}^{\circ} T S_{\beta}$. D'après le cor. $63^{\mathrm{a}}$ ces inclusions impliquent les formules cherchées.

Je vais démontrer à présent deux lemmes qui me permettrons de renforcer le résultat $d u$ th. fond. $\mathrm{XI}^{\mathrm{b}}$ dans le cas où, en dehors $\mathrm{du}$ nombre $\beta$, le nombre $\gamma$ vérifie aussi la formule: $\gamma \leqslant p(a)$.

Lemme 65. Si $\overline{\overline{\boldsymbol{K}}}=\aleph_{\alpha}$, il existe une classe $\boldsymbol{L}$ telle que $\boldsymbol{L} \in \boldsymbol{V}(\boldsymbol{K})$. - $\mathcal{Q}\left(\boldsymbol{S}_{p(a)} \stackrel{+}{\left.S_{p(a)}^{*}\right)}\right.$ et $\overline{\overline{\boldsymbol{L}}}=\boldsymbol{\aleph}_{\alpha}$.

Démonstration. En raison du th. $50^{\text {a }}$ et du lem. 19', on

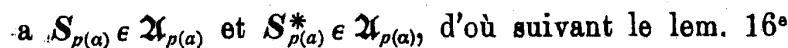

$$
\boldsymbol{S}_{p(a)}+\boldsymbol{S}_{p(a)}^{*} \in \mathfrak{X}_{p(a)}
$$

D'après le th. $50^{\mathrm{f}}$ et le lem. $19^{1}$ (pour $f(\overline{\overline{\boldsymbol{X}}})=(\overline{\overline{\boldsymbol{X}}})^{\alpha_{\beta}}$ ) on a ensuite: $\overline{\overline{\boldsymbol{S}_{p(\alpha)}(\overline{\boldsymbol{X}})}} \leqslant(\overline{\overline{\boldsymbol{X}}})_{p(\alpha)}^{\boldsymbol{N}_{p(\alpha)}}$ et $\overline{\overline{\boldsymbol{S}_{p(\alpha)}^{*}(\overline{\boldsymbol{X}})}} \leqslant(\overline{\overline{\boldsymbol{X}}})^{k_{p(a)}}$, d'où conformément à la déf. $9^{\mathrm{b}} \overline{\overline{\left.\boldsymbol{S}_{p(\alpha)}+\boldsymbol{S}_{p(\alpha)}^{*}\right](\overline{\boldsymbol{X}})}} \leqslant \overline{\overline{S_{p(\alpha)}(\overline{\boldsymbol{X}})}}+\overline{\overline{S_{p(\alpha)}^{*}(\overline{\boldsymbol{X}})}} \leqslant(\overline{\overline{\boldsymbol{X}}})_{p(\alpha)}^{\mathrm{x}_{p}} \cdot 2$ pour toute classe $\boldsymbol{X}$. Si en outre $\overline{\bar{X}} \geqslant 2$, il résulte du lem. $5^{\text {da }}$ que le

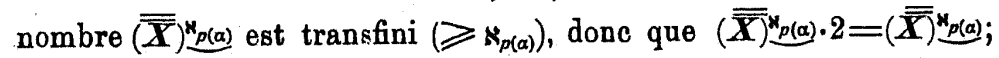
par conséquent, on a dans ce cas:

$$
\overline{\overline{\left[\boldsymbol{S}_{p(\alpha)}+\boldsymbol{S}_{p(\alpha)}^{*} \mid(\overline{\boldsymbol{X}})\right.}} \leqslant(\overline{\overline{\boldsymbol{X}}})^{x_{p(\alpha)}} \text { pour toute classe } \boldsymbol{X} \text {. }
$$

Or, si $\overline{\bar{X}}<2$, les th. 50, a, $.54^{a}$ et le lem. $19^{k}$ impliquent que $\boldsymbol{S}_{p(\alpha)}(\boldsymbol{X})=\boldsymbol{X}=\boldsymbol{S}_{p(\alpha)}^{*}(\boldsymbol{X})$, d'où $\left[\boldsymbol{S}_{p(\alpha)}+\boldsymbol{S}_{p(\alpha)}^{*}\right](\boldsymbol{X})=\boldsymbol{X} ;$ on en conclut sans peine (á l'aide du lem. $5^{\circ}$ ) que la formule (2) est remplie dans. ce cas aussi.

A la suite de (1), (2) et da lem. $4^{\text {b }}$ les hypothèses du th. 31, si l'on y pose: $\boldsymbol{F} \doteq \boldsymbol{S}_{p(\alpha)}+S_{p(a)}^{*}$ et $\beta=p(\alpha)$, se trouvent remplies; 
il en résulte l'existence d'une classe $\boldsymbol{L}$ qui vérifie les formules:

$$
\boldsymbol{L}_{\epsilon} \boldsymbol{V}(\boldsymbol{K}) \cdot \mathcal{e}\left(\boldsymbol{S}_{p(\alpha)}+\boldsymbol{S}_{p(\alpha)}^{*}\right)
$$

$$
\overline{\bar{L}} \leqslant(\overline{\overline{\boldsymbol{K}}})^{\mathrm{N} p(\alpha)} \text {. }
$$

En vertu de la déf. $5^{c}$ la formule (3) donne: $\boldsymbol{K} \subset \boldsymbol{L}$; comme par hypothèse $\overline{\bar{K}}=\aleph_{\alpha}$, on en obtient: $\aleph_{\alpha} \leqslant \overline{\bar{L}}$. D'autre part il résulte du lem $6^{\text {a }}$ que $(\overline{\bar{K}})^{N_{p(\alpha)}}=\aleph_{\alpha}^{N_{p(\alpha)}}=\aleph_{\alpha}$, d'où selon (4) $\overline{\overline{\boldsymbol{L}}} \leqslant \kappa_{\alpha}$. En rapprochant ces deux inégalités, on parvient à la formule:

(5)

$$
\overline{\overline{\boldsymbol{L}}}=\kappa_{\alpha} \text {. }
$$

Les formules (3) et (5) prouvent que $L$ est la classe cherchée.

On peut généraliser et compléter le lemme précédent comme suit: A. Si $\overline{\overline{\boldsymbol{K}}}=\aleph_{\alpha}, \beta \leqslant p(u)$ et $\gamma \leqslant p(a)$, il existe une classe $\boldsymbol{L}$ telle que $\boldsymbol{L} \in \boldsymbol{V}(\boldsymbol{K}) \cdot \mathcal{C}\left(\boldsymbol{S}_{\beta}+\boldsymbol{S}_{\gamma}^{*}\right), \boldsymbol{V}(\boldsymbol{K}) \cdot \mathcal{e} \mathcal{\ell}\left(\boldsymbol{S}_{\beta}+\boldsymbol{S}_{\gamma}^{*}\right) \subset \boldsymbol{V}(\boldsymbol{L})$ et $\overline{\overline{\boldsymbol{L}}}=\aleph_{\alpha}$.

Cette dernière proposition embrasse comme un cas particulier le théorème connu, suivant lequel la classe de tons les ensembles boreliens de nombres réels (ou bien de points d'un espace eucliedien quelconque) est de la puissance $2^{N_{0}}$. Soient, en effet, $\boldsymbol{K}$ la classe de tous les intervalles, $\aleph_{a}=2^{w_{0}}$ et $\beta=\gamma=1$; les hypothèses du th. A sont alors remplies (eu particulier, les inégalités $\beta \leqslant p(\alpha)$ et $\gamma \leqslant p(\alpha)$ résultent du lem. $4^{c}$, et on s'aperçoit facilement que la classe $\boldsymbol{L}$ coïncido avec celle des ensembles boreliens.

Le th. A pent être généralisé à son tour de façon suivante:

B. Si $f(\max (\beta, \gamma))=\max (\beta, \gamma)$, alors à toute classe d'ensembles $\boldsymbol{K}$ correspond une classe $\boldsymbol{L}$ vérifiant les formules: $\boldsymbol{L} \in \boldsymbol{V}(\boldsymbol{K}) \cdot \mathcal{C}\left(\boldsymbol{S}_{\beta}+\boldsymbol{S}_{\gamma}^{*}\right)$, $\boldsymbol{V}(\boldsymbol{K}) \cdot \mathcal{C}\left(\boldsymbol{S}_{\beta}+\boldsymbol{S}_{\gamma}^{*}\right) \subset \boldsymbol{V}(\boldsymbol{L})$ et $\overline{\overline{\boldsymbol{L}}} \leqslant(\overline{\overline{\boldsymbol{K}}})^{\mathbf{w}_{\max (\beta, \gamma)}}$.

De même que le lem. 65 , ce th. $B$ résulte facilement dn th. 31 . Quant an cas où $\operatorname{cf}(\max (\beta, \gamma))<\max (\beta, \gamma)$, of. remarque, p. 222

Lemme 66. Si $\boldsymbol{K}_{\epsilon} \mathcal{Q} \mathcal{P}\left(\boldsymbol{T}+\boldsymbol{S}_{\beta}\right), \boldsymbol{L}_{\epsilon} \mathcal{Q} \mathcal{P}\left(\boldsymbol{S}_{\beta}+\boldsymbol{S}_{\beta}^{*}\right)$ et $\boldsymbol{M}=$ $=\boldsymbol{K}+\boldsymbol{L}+\underset{X+Y}{E}\left[X_{\in} \boldsymbol{K}\right.$ et $\left.Y_{\epsilon} L\right]$, on a $\boldsymbol{M}_{\epsilon} \mathcal{Q} \mathcal{P}\left(\boldsymbol{S}_{\beta}+\boldsymbol{S}_{\beta}^{*}\right)$.

Démonstration. Conformément au th. $21^{\mathrm{b}}$ et à la déf. 16, les. hypothèses du lemme impliquent que

$$
\begin{array}{ccc}
\boldsymbol{X}(\boldsymbol{K}) \subset \boldsymbol{K} & \text { et } & \boldsymbol{S}_{\beta}(\boldsymbol{K}) \subset \boldsymbol{K}, \\
\boldsymbol{S}_{\beta}(\boldsymbol{L}) \subset \boldsymbol{L} & \text { et } & \boldsymbol{S}_{\beta}^{*}(\boldsymbol{L}) \complement_{-} \boldsymbol{L} .
\end{array}
$$

A l'aide de l'axiome du choix on peut faire correspondre à tout ensemble $Z$ de la classe $M$ des ensembles $F(Z)$ et $G(Z)$ de façon que l'on ait

$$
\text { (3) } \quad Z=F(Z)+G(Z), \underset{\text { pour } Z \in \boldsymbol{Z} \boldsymbol{M}}{F(Z)} \operatorname{K}+\{0\} \text { et } G(Z) \epsilon \boldsymbol{L}+\{0\}
$$

(si, en effet $Z \in \boldsymbol{K}$, on pose: $F(Z)=Z$ et $G(Z)=0$; si $Z \in \boldsymbol{L}$, on pose: $F(Z)=0$ et $G(Z)=Z$; enfin, dans le cas qui reste: $\underset{X+\boldsymbol{Y}}{E}\left[X_{\epsilon} \boldsymbol{K}\right.$ et $\left.Y \in \boldsymbol{L}\right]$, on applique l'axiome du choix).

Envisageons une classe arbitraire $N$ vérifiant la formule:

$$
\boldsymbol{N} \in \boldsymbol{U}_{\beta}(\boldsymbol{M})-\{0\}
$$

d'où, en raison de la déf. $5^{\mathrm{b}}$,

$$
\boldsymbol{N} \subset \boldsymbol{M}, \quad \overline{\bar{N}}<\aleph_{\beta} \text { et } \boldsymbol{N} \neq 0 .
$$

Il rèsulte de $(3)$ et $(5)$ que $\bar{F}(\boldsymbol{N}) \subset \boldsymbol{K}+\{0\}, \bar{F}(\boldsymbol{N}) \neq 0$, $\bar{G}(\boldsymbol{N}) \subset \boldsymbol{L}+\{0\}$ et $\bar{G}(\boldsymbol{N}) \neq 0$; on a de plus, en vertu du lem. 11a, $\overline{\bar{F}(N)} \leqslant \overline{\bar{N}}$ et $\overline{\overline{\bar{G}(N)}} \leqslant \overline{\bar{N}}$, d'où selon $(5) \overline{\overline{\bar{F}(\boldsymbol{N})}}<\aleph_{\beta}$ et $\overline{\overline{\overline{G(N)}}}<\aleph_{\beta}$. Par conséquent,

(6) $\bar{F}(\boldsymbol{N}) \epsilon \boldsymbol{U}_{\beta}(\boldsymbol{K}+\{0\})-\{0\}$ et $\bar{G}(\boldsymbol{N}) \epsilon \boldsymbol{U}_{\beta}(\boldsymbol{L}+\{0\})-\{0\}$.

Suivant (6) $\sum_{Z \in \boldsymbol{N}} F(Z)=\Sigma \bar{F}(\boldsymbol{N}) \in \overline{\boldsymbol{\Sigma}}\left(\boldsymbol{U}_{\beta}(\boldsymbol{K}+\{0\}-\{0\})\right.$, done d'après la déf. 20 et le th. $50^{\ominus} \sum_{Z \in N} F(Z) \epsilon S_{\beta}(K+\{0\})=S_{\beta}(K)+\{0\}$; tenant compte de (1), on en obtient:

$$
\sum_{\boldsymbol{Z} \in \boldsymbol{N}} F(Z) \epsilon \boldsymbol{K}+\{0\}
$$

D'une façon complètement analogue on déduit de (6) et (2):

$$
\sum_{Z \in \boldsymbol{N}} G(Z) \epsilon \boldsymbol{L}+\{0\}
$$

Remarquons encore la conséquence suivante de (3) et (5):

(9) $\quad \boldsymbol{\Sigma}(\boldsymbol{N})=\sum_{Z \in \boldsymbol{N}} Z=\sum_{Z \in \boldsymbol{N}}(F(Z)+G(Z))=\sum_{Z \in \boldsymbol{N}} F(Z)+\sum_{Z \in \boldsymbol{N}} G(Z)$. 
Les formules (7)-(9) montrent que l'ensemble $\boldsymbol{Z ( N )}$ pent être présenter sous la forme: $\boldsymbol{\Sigma}(\boldsymbol{N})=X+Y$, où $X_{\epsilon} \boldsymbol{K}+\{0\}$ et $Y_{\epsilon} \boldsymbol{L}+\{0\}$. On a done: $\boldsymbol{\Sigma}(\boldsymbol{N}) \underset{X+Y}{\in} \underset{X+Y}{E}\left[X \boldsymbol{K}+\{0\}\right.$ et $\left.Y_{\epsilon} \boldsymbol{L}+\{0\}\right]=\underset{X+Y}{E}\left[X_{\epsilon} \boldsymbol{K}\right.$ et $\left.Y_{\epsilon} \boldsymbol{L}\right]+$ $+\boldsymbol{K}+\boldsymbol{L}+\{0\}$, d'où en vertu de l'hypothèse $\Sigma(\boldsymbol{N}) \in \boldsymbol{M}+\{0\}$, c.-̀े.d. soit $\boldsymbol{\Sigma}(\boldsymbol{N}) \epsilon \boldsymbol{M}$, soit $\boldsymbol{\Sigma}(\boldsymbol{N}) \epsilon\{0\}$. Or, il est à observer que la formule: $\boldsymbol{\Sigma}(\boldsymbol{N}) \epsilon\{0\}$ entraîne selon (5): $\boldsymbol{N}=\{0\},\{0\} \subset \boldsymbol{M}$, donc aussi $\Sigma(\boldsymbol{N}) \in \boldsymbol{M}$. Par conséquent, on a en tout cas

$$
\Sigma(\boldsymbol{N}) \in \boldsymbol{M} \text {. }
$$

Passons à présent à l'ensemble $\Pi(N)$. La formule aisée à établir de l'algèbre de la logique: $\prod_{x \in A}(F(x)+G(x))-\prod_{x \in A} G(x) \subset$ $C \sum_{x \in A} F(x)$ permet de déduire de (3) et (5) que

(11) $\Pi(N)-\prod_{Z \in N} G(Z)=\prod_{Z \in \boldsymbol{N}}\left(F^{\prime}(Z)+G(Z)\right)-\prod_{Z \in \boldsymbol{N}} G(Z) \subset \sum_{Z \in \boldsymbol{N}} F(Z)$.

Il résulte de (7) et (11) que $\Pi(\boldsymbol{N})-\prod_{Z \in N} G(Z) \epsilon \sum_{X_{\epsilon} \boldsymbol{K}+\{0\}} U(X)$, d'où d'après la dét. $17 \Pi(N)-\prod_{Z \in N} G(Z)_{\epsilon} \boldsymbol{T}((\boldsymbol{K})+\{0\})$. Or, le lem. $15^{\mathrm{b}}$ et le th. 36,ad impliquent l'identité: $\boldsymbol{T}(\boldsymbol{K}+\{0\})=\boldsymbol{T}(\boldsymbol{K})+\boldsymbol{T}(\{0\})=$ $=\boldsymbol{T}(\boldsymbol{K})+\{0\}$. Il s'en suit que $\Pi(\boldsymbol{N})-\prod_{Z_{\in} \boldsymbol{N}} G(Z) \in \boldsymbol{T}(\boldsymbol{K})+\{0\}$, d'où selon (1)

$$
\Pi(\boldsymbol{N})-\prod_{\boldsymbol{Z} \in \boldsymbol{N}} G(\boldsymbol{Z}) \epsilon \boldsymbol{K}+\{0\}
$$

D'autre part on a en vertu de (6): $\bar{\Pi} G(\boldsymbol{N}) \epsilon \bar{\Pi}\left(\boldsymbol{U}_{\beta}(\boldsymbol{L}+\{0\})-\{0\}\right)$, donc d'après les th. 53 et $54^{\mathrm{b}} \prod_{Z \in N} G(Z)=\Pi \bar{G}(N) \in S_{\beta}^{*}(L+\{0\})=$
$=S^{*}(\boldsymbol{L})+\{0\}$. On en obtient suivant $(2)$ :

$$
\prod_{Z \in \boldsymbol{N}} G(Z) \epsilon \boldsymbol{L}+\{0\} \text {. }
$$

Observons enfin que les formules (3) et (5) entraînent l'inclusion: $\prod_{Z \in N} G(Z) \subset \Pi(\mathbf{N})$, qui donne à son tour l'égalité:

$$
\Pi(\boldsymbol{N})=\left(\Pi(\boldsymbol{N})-\prod_{Z \in \boldsymbol{N}} G(Z)\right)+\prod_{Z \in \boldsymbol{N}} G(Z) .
$$

Ainsi nous avons acquis dans les formules (12)-(14) une représentation de l'ensemble $\Pi(\boldsymbol{N})$ sous la forme: $\Pi(\boldsymbol{N})=X+Y$, ou $X_{\epsilon} \boldsymbol{K}+\{0\}$ et $Y_{\epsilon} \boldsymbol{L}+\{0\}$; tout comme auparavant (pour l'ensemble $\boldsymbol{\Sigma}(\boldsymbol{N}))$ nous en concluons que $\Pi(\boldsymbol{N}) \in \boldsymbol{M}+\{0\}$, donc que $\Pi(\boldsymbol{N}) \in \boldsymbol{M}$ ou bien $\Pi(\boldsymbol{N}) \in\{0\}$. Or, le cas de $\Pi(\boldsymbol{N}) \in\{0\}$ peut etre éliminé de façon suivante: si $\boldsymbol{K} \neq 0$, on a en conséquence du th. 36 $0 \in \boldsymbol{\epsilon}(\boldsymbol{K})$, donc selon (1) $0 \in \boldsymbol{K}, \Pi(\boldsymbol{N}) \epsilon\{0\} \subset \boldsymbol{K}$, d'où à fortiori $\Pi(\boldsymbol{N}) \in \boldsymbol{M}$ ( $\boldsymbol{K}$ étant par hypothèse une sous-classe de $\boldsymbol{M}$ ); si par contre $\boldsymbol{K}=0$, la classe $M$ coïncide avec $L$, donc en vertu de (4) $N_{\epsilon} U_{\beta}(L)-\{0\}$ et, en raison du th. $53, \Pi(\boldsymbol{N}) \in S_{\beta}^{*}(\mathcal{L})$, d'où enfin selon (2) $\Pi(\boldsymbol{N}) \in \boldsymbol{L}=\boldsymbol{M}$. Ainsi la formule: $\Pi(N) \in\{0\}$ entraîne toujours: $\Pi(N) \in \boldsymbol{M}$; par conséquent,

$$
\Pi(\boldsymbol{N}) \in \boldsymbol{M} \text {. }
$$

Il est donc prouvé que la formule (4) implique constamment (10) et (15). On en obtient les inclusions: $\bar{\Sigma}\left(\boldsymbol{U}_{\beta}(\boldsymbol{M})-\{0\}\right) \subset \boldsymbol{M}$ et $\bar{\Pi}\left(\boldsymbol{U}_{\beta}(\boldsymbol{M})-\{0\} \subset \boldsymbol{M}\right.$, qui, d'après la déf. 20 et le th. 53, peuvent être exprimées plus court:

$$
\boldsymbol{S}_{, \beta}(\boldsymbol{M}) \subset \boldsymbol{M} \text { et } \boldsymbol{S}_{\beta}^{*}(\boldsymbol{M}) \subset \boldsymbol{M} \text {. }
$$

Conformément à la définition 16 , les formules (16) donuent: $M_{\epsilon} \mathcal{C} \mathcal{C}\left(\boldsymbol{S}_{\beta}\right) \cdot \mathcal{C} \mathcal{P}\left(\boldsymbol{S}_{\beta}^{*}\right)$; à l'aide du th. $21^{\mathrm{b}}$ on en conclut aussitôt que

$$
\boldsymbol{M}_{\in} \in \mathcal{Q}\left(\boldsymbol{S}_{\beta}+\boldsymbol{S}_{\beta}^{*}\right) \text {, c. q. f. d. }
$$

En analysant cette démonstration, il est facile d'en tirer le lemme suivant:

Soit $\boldsymbol{K} \stackrel{*}{+} \boldsymbol{L}=\boldsymbol{K}+\boldsymbol{L}+\underset{X+Y}{E}[X \in \boldsymbol{K}$ et $Y \in \boldsymbol{L}]$ pour toutes deux classes $\boldsymbol{K}$ et $L ;$ on a alors $\dot{S}_{\beta}\left(\boldsymbol{K}^{*} \boldsymbol{L}\right)=\boldsymbol{S}_{\beta}(\boldsymbol{K}) \stackrel{*}{+} \boldsymbol{S}_{\beta}(\boldsymbol{L})$ et $\boldsymbol{S}_{\beta}^{*}(\boldsymbol{K} \stackrel{*}{+} \boldsymbol{L}) \subset \boldsymbol{T} \boldsymbol{S}_{\beta}(\boldsymbol{K}) \stackrel{*}{+} \boldsymbol{S}_{\beta}^{*}(\boldsymbol{L})$. 
On pent démontrer que la classe $\boldsymbol{M}$ examinée dans le lem. 66 est la plus petite classe contenant les classes données $\boldsymbol{K}$ et $\boldsymbol{L}$ et close par rapport à l'opération $S_{\beta}+S_{\beta}^{*}$ (ou - ce qui revient au même par rapport anx deax opérations $\boldsymbol{S}_{\beta}$ et $\boldsymbol{S}_{\beta}^{*}$ à la fois); elle se laisse done définir par la formule: $\boldsymbol{M}=\Pi\left(\boldsymbol{V}(\boldsymbol{K}+\boldsymbol{L}) \cdot \mathcal{Q} \mathcal{\ell}\left(\boldsymbol{S}_{\beta}+\boldsymbol{S}_{\beta}^{*}\right)\right)$. L'existence d'une classe $\boldsymbol{M}$ jouissant de ces propriétés se déduit dans le cas général du cor. 30 ou bien du th. 31 ; cependant, dans le cas particulier envisagé ici, à canse des fortes hypothèses faites sur les classes $\boldsymbol{K}$ et $\boldsymbol{L}$, la structure de la classe $\boldsymbol{M}$ est particalièrement simple.

Théorème 67. Si $\overline{\overline{1}}=\aleph_{\alpha}=\overline{\overline{\boldsymbol{K}}}$ (oì $\left.\boldsymbol{K} \subset \boldsymbol{U}(1)\right), \quad \beta \leqslant p(\alpha)$ et

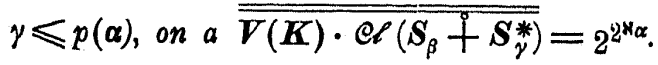

Démonstration. D'après le lem. 65 il existe une classe $\boldsymbol{C}$ vérifiant les formules:

et

$$
\boldsymbol{L}_{\in} \boldsymbol{V}(\boldsymbol{K}) \cdot \mathcal{C} \mathcal{P}\left(\boldsymbol{S}_{p(\alpha)}+\boldsymbol{S}_{p(\alpha)}^{*}\right)
$$

$$
\overline{\bar{L}}=\aleph_{\alpha} .
$$

Posons pour toute classe d'ensembles $\boldsymbol{M}$ :

$$
\begin{gathered}
\boldsymbol{F}(\boldsymbol{M})=\boldsymbol{L}+\boldsymbol{M}+\underset{\boldsymbol{X}+\boldsymbol{Y}}{E}\left[X \in \boldsymbol{L} \text { et } Y_{\epsilon \in \boldsymbol{M}}\right], \\
\boldsymbol{G}(\boldsymbol{M})=\boldsymbol{L} \cdot \boldsymbol{M} .
\end{gathered}
$$

Envisageons deux classes arbitraires $M$ et $N$ telles que

$$
\begin{aligned}
& M_{\epsilon} \mathcal{E} \mathcal{P}\left(\boldsymbol{T}+\boldsymbol{S}_{p(\alpha)}\right) \text { et } \boldsymbol{N}_{\boldsymbol{\epsilon}} \mathcal{E} \mathcal{P}\left(\boldsymbol{T}+\boldsymbol{S}_{p(\alpha)}\right), \\
& \boldsymbol{F}(\boldsymbol{M})=\boldsymbol{F}(\boldsymbol{N}) \text { et } \boldsymbol{G}(\boldsymbol{M})=\boldsymbol{G}(\boldsymbol{N}) \text {. }
\end{aligned}
$$

Nous allons montrer que

$$
\boldsymbol{M} \cdot \underset{X+Y}{E}\left[X \in L \text { et } \quad Y_{\in} \boldsymbol{N}\right] \subset \boldsymbol{N}
$$

Soit, en effet,

et

$$
Z \in M
$$

$$
Z=X+Y, \text { ò̀ } X_{\epsilon} L \text { et } Y_{\epsilon} \boldsymbol{N} \text {. }
$$

En vertu de (9) $X \subset Z$, d'où selon (8) et d'après la déf. 17 $X \in \boldsymbol{T}(\boldsymbol{M})$. Comme, en raison du th. $21^{\mathrm{b}}$ et de la déf. 16 , (5) donne:
$\boldsymbol{T}(\boldsymbol{M}) \subset \boldsymbol{M}$, on a ensuite $X \in \boldsymbol{M}$, d'où suivant (9) $X_{\epsilon} \boldsymbol{L} \cdot \boldsymbol{M}$; en tenant compte de (4) et (6), on en obtient: $X_{\epsilon} L \cdot N . Y$ appartenant a $\boldsymbol{N}$ en vertu de (9), on a par conséquent $\{X, Y\} \subset \boldsymbol{N}$; comme en outre $\left\{\overline{\overline{X, Y}\}}<\aleph_{p(a)}\right.$ et $\{X, Y\} \neq 0, \quad$ on en conclut que $\{X, Y\} \in \boldsymbol{U}_{p(a)}(\boldsymbol{N})-\{0\}$, d'où, conformément à la déf. $20($ pour $\boldsymbol{K}=\boldsymbol{N})$, $Z=\Sigma(\{X, Y\}) \in \bar{\Sigma}\left(U_{p(a)}(N)-\{O\}\right)=S_{p(a)}(N)$. Notons encore que $S_{p(a)}(N) \subset N$ (en conséquence de (6), du th. $21^{\text {b }}$ et de la déf. 16). On parvient ainsi à la formule:

$Z \in \boldsymbol{N}$.

Il est donc prouvé que les conditions (8) et (9) impliquent tonjours (10); par conséquent, l'inclusion (7) se trouve établie.

Remarquons maintenant qu'en vertu de (6) et (4) l'inclusion suivante se vérifie aussi:

$$
\boldsymbol{M} \cdot(\boldsymbol{L}+\boldsymbol{N}) \subset \boldsymbol{N} .
$$

Les formules (7) et (11) donnent: $\boldsymbol{M} \cdot(\boldsymbol{L}+N+\underset{X+Y}{E}[X \in \boldsymbol{L}$ et $\left.\left.Y_{\epsilon} \boldsymbol{N}\right]\right) \subset N$; si.l'on remplaçe dans (3) $\boldsymbol{M} \operatorname{par} \boldsymbol{N}$, on en conclut que $\boldsymbol{M} \cdot \boldsymbol{F}(\boldsymbol{N}) \subset \boldsymbol{N}$, d'où selon (6) $\boldsymbol{M} \cdot \boldsymbol{F}(\boldsymbol{M}) \subset \boldsymbol{N}$. Comme de plus, en raison de (3), $\boldsymbol{M} \subseteq \boldsymbol{F}(\boldsymbol{M})$, on a finalement

$$
\boldsymbol{M} \subset \boldsymbol{N} \text {. }
$$

L'inclusion inverse: $\boldsymbol{N} \subset \boldsymbol{M}$ se déduit d'une façon complètement analogue; en la rapprochant de (12), on obtient done l'égalité :

$$
\boldsymbol{M}=\boldsymbol{N} \text {. }
$$

Nous avons ainsi montré que les formules (5) et (6) entraînent constamment (13). Ce résultat peut être évidemment formulé de la façon suivante: $\boldsymbol{Y}$ étant une classe quelconque, si $\boldsymbol{M} \in \mathcal{C} \ell\left(\boldsymbol{T}+\boldsymbol{S}_{p(a)}\right)$. $\underset{X}{E}[\boldsymbol{F}(X)=\boldsymbol{Y}], \boldsymbol{N}_{\in} \mathfrak{C}_{t}\left(\boldsymbol{T}+S_{p(a)}\right) \cdot \underset{\boldsymbol{X}}{E}[\boldsymbol{F}(\boldsymbol{X})=\boldsymbol{Y}]$ et $\boldsymbol{G}(\boldsymbol{M})=G(\boldsymbol{N})$, on a $M=N$; autrement dit, la fonction $G$ transforme d'une façon biunivoque la famille $\mathcal{C}\left(\boldsymbol{T}+\boldsymbol{S}_{p(a)}\right) \cdot \underset{\boldsymbol{X}}{E}[\boldsymbol{F}(\boldsymbol{X})=\boldsymbol{Y}]$ en son image suivant $G$, c.-à-d. en famille $\overline{\boldsymbol{X}}\left(\mathcal{C} \ell\left(\boldsymbol{T}+\boldsymbol{S}_{p(\alpha)}\right) \cdot \underset{\boldsymbol{X}}{E}[\boldsymbol{F}(\boldsymbol{X})=\boldsymbol{Y})\right.$. Par conséquent, ces deux familles ont la même puissance:

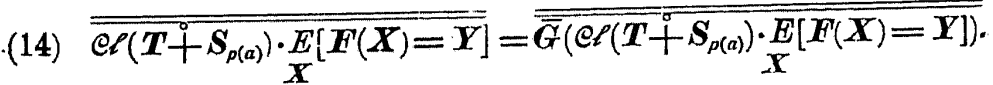


Remarquons qu'en vertu de (4) $\bar{G}(\mathscr{F}) \subset \boldsymbol{U}(\boldsymbol{L})$ pour toute $\mathrm{f}_{\mathrm{a}}$ mille de classes $\mathscr{F}$, d'où selon (2) et d'après le lem. $10^{\mathrm{a}} \overline{\overline{\bar{G}(\mathscr{C H})}} \leqslant 2^{\mathrm{N \alpha}}$. Cette inégalité se vérifie, en particulier, pour $\mathscr{T}=\mathfrak{e} l\left(\boldsymbol{T}+\boldsymbol{S}_{p(a)}\right)$. - $\underset{X}{\boldsymbol{E}}[\boldsymbol{F}(\boldsymbol{X})=\boldsymbol{Y}]$, ce qui, rapproché de (14), donne:

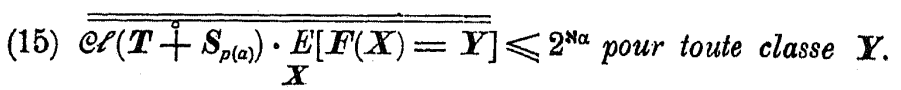

Conformément au th. fond. $\mathrm{X}$ (pour $\beta=p(a)$ ), on a

$$
\overline{\overline{\mathscr{Q} P}\left(\boldsymbol{T}+\boldsymbol{S}_{p(\alpha)}\right.}=2^{2^{\boldsymbol{N}_{\alpha}},}
$$

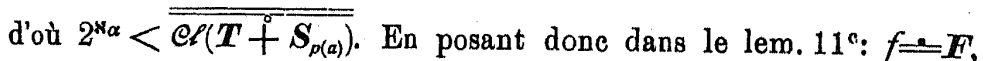
$A=\mathcal{C}\left(\boldsymbol{T}+\boldsymbol{S}_{p(a)}\right)$ et $\mathfrak{b}=2^{\mathrm{N}_{\alpha}}$ et en tenant compte de (15), on s'apercoit aussitôt que les hypothèses de ce lemme sont remplies. Par conséquent, $\overline{\overline{\boldsymbol{F}}\left(\mathcal{C} \mathcal{C}\left(\boldsymbol{T}+\boldsymbol{S}_{p(a)}\right)\right)}=\overline{\overline{\mathcal{C} \ell\left(T+S_{p(a)}\right)}}$, d'où suivant (16)

$$
\overline{\overline{\boldsymbol{F}\left(\mathcal { C } \mathcal { P } \left(\boldsymbol{T}+\overline{\left.\left.S_{p(a)}\right)\right)}\right.\right.}}=2^{2^{\alpha \alpha}} .
$$

Considérons une c̀lasse quelconque $\boldsymbol{M}$ de $\mathcal{C} \mathcal{C}\left(\boldsymbol{T}+\boldsymbol{S}_{p(a)}\right)$. En raison de (1) et de (3), le lem. 66, si l'on y remplace $\boldsymbol{K}$ par $\boldsymbol{M}, \boldsymbol{M}$ par $\boldsymbol{F}(\boldsymbol{M})$ et $\beta$ par $p(\alpha)$, nous donne: $\boldsymbol{F}(\boldsymbol{M}) \in \mathcal{Q} \mathcal{\ell}\left(\boldsymbol{S}_{p(\alpha)}+\boldsymbol{S}_{p(a)}^{*}\right)$; il résulte en outre de (1), (3) et de la déf. $5^{\circ}$ que $\boldsymbol{K} \subset \boldsymbol{L} \subset \boldsymbol{F}(\boldsymbol{M})$, donc que $\boldsymbol{F}(\boldsymbol{M}) \in \boldsymbol{V}(\boldsymbol{K})$. Or, ce raisonnement s'appliquant à toute classe $\boldsymbol{M}$ de $\mathcal{Q}\left(\boldsymbol{T}+\boldsymbol{S}_{p(a)}\right)$, on conclut que

$$
\overline{\boldsymbol{F}}\left(\mathcal{Q} \mathcal{L}\left(\boldsymbol{T}+\boldsymbol{S}_{p(a)}\right) \subset \boldsymbol{V}(\boldsymbol{K}) \cdot \mathcal{C} \mathcal{L}\left(\boldsymbol{S}_{p(a)}+\boldsymbol{S}_{p(a)}^{*}\right) .\right.
$$

Conformément au th. $52^{\text {b }}$, les inégalités: $\beta \leqslant p(a)$ et $\gamma \leqslant p(a)$, données par l'hypothèse, impliquent que $\boldsymbol{S}_{\beta} \check{\complement} \boldsymbol{S}_{p(a)}$ et $\boldsymbol{S}_{\gamma} \check{\complement} \boldsymbol{S}_{p(a)}$,

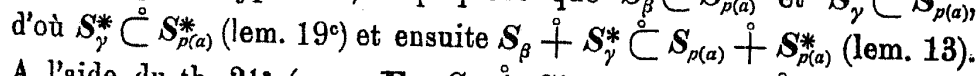
A l'aide du th. $21^{a}$ (pour $\boldsymbol{F}=S_{\beta}+S_{\gamma}^{*}$ et $G=S_{p(a)}+S_{p(a)}^{*}$ ) on en obtient: $\mathscr{C}\left(\boldsymbol{S}_{\boldsymbol{p}(a)}+\boldsymbol{S}_{p(a)}^{*}\right) \subset \mathcal{C} \mathcal{C}\left(\boldsymbol{S}_{\beta}+\boldsymbol{S}_{\gamma}^{*}\right)$, d'où selon (18)

$$
\overline{\boldsymbol{F}}\left(\mathcal{C} \mathcal{P}\left(\boldsymbol{T}+\boldsymbol{S}_{p(\alpha)}\right) \subset \boldsymbol{V}(\boldsymbol{K}) \cdot \mathcal{e}\left(\boldsymbol{S}_{\beta}+\boldsymbol{S}_{\gamma}^{*}\right) .\right.
$$

Or, les formules (17) et (19) entraînent tout de suile:

$$
\left.\overline{\overline{\boldsymbol{V}(\boldsymbol{K}) \cdot \mathcal{C} \ell\left(\boldsymbol{S}_{\beta}+\boldsymbol{S}_{\gamma}^{*}\right.}}\right) \geqslant 2^{2^{\mathrm{N}_{\alpha}}} \text {. }
$$

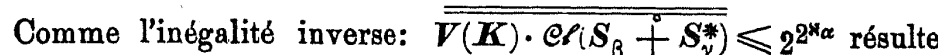
immédiatement du th. fond. $\mathrm{XI}^{\mathrm{b}}$ (ou bien du th. 32), on a finalement:

$$
\overline{\overline{\boldsymbol{V}(\boldsymbol{K}) \cdot \mathcal{C} \mathcal{\ell}\left(\boldsymbol{S}_{\beta}+\boldsymbol{S}_{\gamma}^{*}\right)}}=2^{2 \mathrm{\alpha} \alpha}, \quad \text { c. q. f. d. }
$$

On peut montrer par un exemple convenablement choisi que les deax inégalités: $\beta \leqslant p(\alpha)$ et $\gamma \leqslant p(\alpha)$, qui figarent dans l'hypothèse de ce dernier théorème, sont essentielles; il s'en suit à plus forte raison que l'opération $S_{\gamma}^{*}$ ne s'y laisse remplacer ni par $S^{*}$ ni par $\boldsymbol{T}$. Il semble, par cońtre, probable, bien que je ne sache pas le démontrer, qu'il est possible de remplacer l'opération $S_{\gamma}^{*}$ dans le th. 67 par $C$ et de renforcer ainsi le th. fond. IX.

Quant à l'opération $\boldsymbol{R}_{\beta}$, un résultat analogue ne se laisse établir pour elle que sous des restrictions supplémentaires, notamment dans la forme du théorème

A. Si $\overline{\overline{1}}=\aleph_{\alpha}=\overline{\bar{K}}$ (oì $\left.\boldsymbol{K} \subset \boldsymbol{U}(1)\right), \beta \leqslant p(\alpha)$ et $2^{2 \alpha_{\beta}} \leqslant \aleph_{a}$, on $a \overline{\overline{\boldsymbol{V}(\boldsymbol{K}) \cdot \mathcal{C} \ell\left(\boldsymbol{R}_{\beta}\right)}}=2^{2^{\mathrm{N} \alpha} \text {. }}$

La démonstration s'appuie sur le th. $55^{\mathfrak{g}}$ et sur deux lemmes suivants, qui se déduisent d'une façon analogue aux lem. 65 et 66 :

B. Si $\overline{\overline{\boldsymbol{K}}}=\aleph_{\alpha}, \quad \beta \leqslant p(\alpha)$ et $2^{2 \boldsymbol{N}_{\beta}} \leqslant \aleph_{\alpha}$, il existe une classe $\boldsymbol{L}$ telle que $\boldsymbol{L} \in \boldsymbol{V}(\boldsymbol{K}) \cdot \mathcal{Q} \mathcal{L}\left(\boldsymbol{R}_{\beta}\right)$ et $\overline{\bar{L}}=\aleph_{\alpha}$.

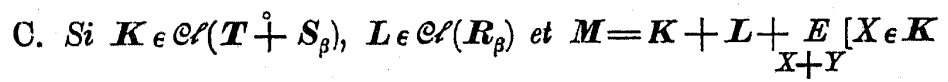

et $\left.Y_{\epsilon} \boldsymbol{L}\right]$, on a $\boldsymbol{M}_{\epsilon} \mathcal{Q P}\left(\boldsymbol{R}_{\beta}\right)$.

Notons que la formule: $\beta \leqslant p(\alpha)$, resp. $\gamma \leqslant p(\alpha)$, est en tout cas remplie, lorsque $\beta=0$, resp. $\gamma=0$; cette remarque permet de déduire $d u$ th. fond. $X$ et du th. 67 quelques corollaires que je ne formulerai pas ici explicitement. Le cas plus intéressant est celai où $\beta$, resp. $\max (\beta, \gamma)$, est un nombre de $1^{\text {ro }}$ espèce et $\boldsymbol{\aleph}=\mathfrak{a}^{\boldsymbol{x} \beta-1}$, resp. $=\mathfrak{a}^{\mathrm{x} \max (\beta, \gamma)-1}$; à cause du lem. $4^{\mathrm{c}}$, les inégalités en question sont vraies aussi dans ce cas. En particulier, on obtient par cette voie le suivant

Corollaire 68. a) Si 1 est l'ensemble de tous les nombres réels (ou, d'une façon plus générale, un ensemble de la puissance $2^{\mathrm{N}_{0}}$ ), on $a \overline{\overline{\mathcal{Q P}\left(T+S_{1}\right)}}=2^{2^{2^{W_{0}}}}$

$\left.{ }^{b}\right)$ si, en outre, $\boldsymbol{K}$ est la classe de tous. les intervalles de nombres réels (ou, plus généralement, une classe quelconque de la puissance $2^{\text {to }_{0}}$ de sous-ensembles de 1), on a $\overline{\overline{V(K)} \cdot \mathcal{C} \ell\left(\boldsymbol{S}_{1}+S_{1}^{*}\right)}=2^{2^{2^{W_{0}}}}$. 


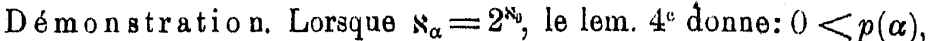
d'où $1 \leqslant p(\alpha)$. Si l'on pose, par conséquent, dans les th. $\mathrm{X}$ et 67 : $\aleph_{\alpha}=\boldsymbol{z}^{\aleph_{0}}$ et $\beta=1$, resp. $\beta=\gamma=1$, les bypothèses de ces théorèmes se trouvent remplies, et on obtient les formules cherchées.

Ce corollaire présente la solution des problèmes qui m'ont été posés par MM. Poprougénko et Sierpiński ${ }^{1}$ ). Comme on voit facilement, la famille $\boldsymbol{V}(\boldsymbol{K}) \cdot \mathcal{e} f\left(\boldsymbol{S}_{1}+\boldsymbol{S}_{1}^{*}\right)$, considérée dans le cor. $68^{\text {b }}$, coïncide avec celle de toutes les classes d'ensembles inductives au sens de M. Lusin ${ }^{2}$; la plus petite classe $\boldsymbol{L}$ de cette famille, $\boldsymbol{L}=\Pi\left(\boldsymbol{V}(\boldsymbol{K}) \cdot \mathcal{Q} \mathcal{P}\left(\boldsymbol{S}_{1}+\boldsymbol{S}_{1}^{*}\right)\right)$, est notoirement celle de tous les ensembles boreliens $\left.{ }^{2}\right)$.

Il est à noter, an sujet dess remarques faites sur le th. 67 (p. 275), que même dans le cas particulier de ce théorème, à savoir daus le cor. $68^{\mathrm{b}}$, l'opération $S_{1}^{*}$ ne peut être remplacée ni par $S^{*}$ ni par $\boldsymbol{T}$ ni par $\boldsymbol{R}_{1}$ (car la famille $\left.\boldsymbol{V}(\boldsymbol{K}) \cdot \mathcal{e} \mathcal{P}_{(} \boldsymbol{S}_{1}+\boldsymbol{S}^{*}\right)=\boldsymbol{V}(\boldsymbol{K}) \cdot \mathcal{C} \mathcal{\ell}\left(\boldsymbol{S}_{\mathbf{1}}+\boldsymbol{T}\right)=$ $=\boldsymbol{V}(\boldsymbol{K}) \cdot \mathcal{O P}\left(\boldsymbol{R}_{1}\right)$, où $\boldsymbol{K}$ est la classe de tous Ies intervalles, ne contient qu'un seul élément, notamment la classe de tous les ensembles de nombres réels). Nous ignorons cependant, si l'on ne peut remplacer $\boldsymbol{S}_{1}^{*}$ par $C$, c.-à-d., si les classes d'ensembles de nombres réels, contenant tous les intervalles, toutes les sommes des infinités dénombrables et tous les complémentaires de ses éléments, forment une famille de puissance $2^{2^{2^{t_{0}}}}$

Or, on peut renforcer le cor. $68^{\mathrm{b}}$, en y remplaçant les opérations $S_{1}$ et $S_{i}^{*}$ par des opérations quelconques d'une vaste classe que M. Ha usdorff appelle $\delta s$ - (resp. od.) Functionen, done p. ex par l'opération $\boldsymbol{A}$ de $M$. Souslin et son opération double $\left.\boldsymbol{A}^{*}\right)^{\text {s) }}$. Par une extension convenable de cette notion de $M$. Ha usdorff on parvient même à une généralisation du th. $67 \%$ ).

Les résultats obtenus jusqu'ici ne nous fournissent aucun moyen d'évaluer la puissance des familles $\mathcal{e} f\left(\boldsymbol{C}+\boldsymbol{S}_{\beta}\right)$, $\mathcal{C}\left(\boldsymbol{T}+\boldsymbol{S}_{\beta}\right)$, $\mathcal{C} \mathcal{C}\left(\boldsymbol{S}_{\beta}+\boldsymbol{S}_{,}^{*}\right)$ etc. dans le cas où le nombre $\beta$ (resp. les deux nombres $\beta$ et $\gamma)$ est $>p(a) \aleph_{\alpha}$ désignant la puissance de l'ensemble universel 1. Ce cas offre des difficultés tout à fait essentielles. A vrai dire, nous ne pourrions aboutir ici à des conclusions défi-

3) Cf. W. Si irpiniski, Sur les familles inductives et projectives d'ensembles, Find. Math. XIII, p. 228.

2) Cf. p. 268.

2) F. H a u s d o rf f, Mengenlehre, Il Aufl., Berlin und Leipzig 1927, p. 89-93.

4) L'extension de certains résultats obtenns dans cet ouvrage aux opórations de M. Haudorff fera objet d'une note spéciale (à paraitre dans ce Journal). nitives que dans le cas où les nombres envisagés $\beta$ et $\gamma$ dépassent non seulement $p(\alpha)$, mais aussi $\alpha$; en effet, à la suite du th. $52^{d}$, les familles en question coïncident alors avec celles qui ont été examinées dans les th. fond. V-VII du § précédent, de sorte que chacune de ces familles est de la puissance $2^{\mathrm{k}_{\alpha}}$. Nous montrerons dans la suite que le même résultat se laisse aussi obtenir dans les hypothèses plus générales : $\beta>p(\alpha)$, resp. $\gamma>p(\alpha)$, à condition toutefois d'admettre comme base des raisonnements l'hypothèse $\mathrm{H}$, c.-à-d. l'hypothèse de Cantor sur les alephs, dont il a été question dans le $\S 1$ (p. 193). A ce but, nous allons déterminer d'abord certaines limites pour les puissances des familles examinées, sans nous servir pour le moment de l'hypothèse $\mathrm{H}$.

Théorème 69. Si $\overline{\overline{1}}=\aleph_{\alpha}, \beta>c f(\alpha)$ et $\gamma>c f(\alpha)$, on a

$$
2^{\boldsymbol{N}_{\alpha}} \leqslant \overline{\overline{\mathcal{C} \mathcal{P}\left(\boldsymbol{S}_{\beta}+\boldsymbol{S}_{\gamma}^{*}\right)}} \leqslant 2^{2^{\mathrm{N} \alpha}} .
$$

Démonstration. En raison du th. $51^{\mathrm{b}}$ et du lem. $19^{\mathrm{b}, \mathrm{a}}$ on a: $\boldsymbol{S}_{\beta} \check{\complement}^{\circ} \boldsymbol{S}$ et $\boldsymbol{S}_{\gamma}^{*} \complement^{\circ} \boldsymbol{S}^{*} \complement^{\circ} \boldsymbol{T}$, d'où $\boldsymbol{S}_{\beta}+\boldsymbol{S}_{\gamma}^{*} \complement^{\circ} \boldsymbol{T}+\boldsymbol{S}$; par l'appli-

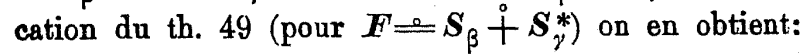

$$
2^{\star \alpha} \leqslant \overline{\overline{\mathcal{Q} \mathcal{\ell}\left(S_{\beta}+S_{\gamma}^{*}\right)}} .
$$

Le lemme $2^{\mathrm{b}}$ (pour $A=1$ ) implique l'existence d'une suite d'ensembles $A_{\xi}$ du type $\omega_{c f(a)}$ vérifiant les formules:

et

$M$ étant une classe quelconque d'ensembles, posons:

$$
\begin{gathered}
\boldsymbol{F}(\boldsymbol{M})=\underset{A_{\xi} \cdot X}{E}\left[\xi<\omega_{c f(a)} \text { et } X \in \boldsymbol{M}\right] . \\
G(\boldsymbol{M})=\boldsymbol{M} \cdot \sum_{\xi<\omega_{c f(a)}} \boldsymbol{U}\left(A_{\xi}\right) .
\end{gathered}
$$

Soient $M$ et $N$ deux classes assujetties aux conditions:
$\boldsymbol{M}_{\epsilon} \mathcal{e} \mathcal{P}\left(\boldsymbol{S}_{\beta}+\boldsymbol{S}_{\gamma}^{*}\right)$ et $\boldsymbol{N} \in \mathcal{Q} \mathcal{P}\left(\boldsymbol{S}_{\beta}+\boldsymbol{S}_{\gamma}^{*}\right)$ 


$$
\boldsymbol{F}(\boldsymbol{M})=\boldsymbol{F}(\boldsymbol{N}) \text { et } \quad \boldsymbol{G}(\boldsymbol{M})=\boldsymbol{G}(\boldsymbol{N}) ;
$$

nous montrerons que ces classes coïncident.

A ce but envisageons un ensemble arbitraire $X$ tel que

$$
X \in \boldsymbol{M} \text {. }
$$

On conclut aisément de $(6),(8)$ et (9) que

$$
\text { la formule: } X \in \sum_{\xi<\omega_{f f i}(\alpha)} U\left(A_{\xi}\right) \text { entraîne: } X \in \boldsymbol{N} \text {. }
$$

Or, admettons dans la suite que

$$
X \overline{\bar{\epsilon}} \sum_{\xi<\omega_{\mathscr{C F}(\alpha)}} \boldsymbol{U}\left(A_{\xi}\right)
$$

On a évidemment selon (2):

$$
X=\sum_{\xi<\omega_{f f(\alpha)}} A_{\xi} \cdot X
$$

Soit $\xi$ un nombre ordinal vérifiant l'inégalité:

$$
\xi<\omega_{f \mathcal{f}(\alpha)} .
$$

Il existe sans doute un nombre $\xi_{1}$ tel que

$$
\xi \leqslant \xi_{1}<\omega_{c f(\alpha)} \text { et } A_{\xi_{1}} \cdot X-\sum_{\eta<\xi_{1}} A_{\eta} \cdot X \neq 0
$$

car dans le cas contraire les formules (3), (12) et (13) donneraient: $X=A_{\xi} \cdot X_{\epsilon} \sum_{\xi<\omega_{c f(a)}} U\left(A_{\xi}\right)$, en contradiction avec (11).

En vertu de (5), (9) et (13) on a $A_{\xi_{1}} \cdot X_{\epsilon} \boldsymbol{F}(\boldsymbol{M})$, d'où selon (8) $A_{\xi_{1}} \cdot X_{\epsilon} \boldsymbol{F}(\boldsymbol{N})$. En appliquant une fois encore la formule (5) (pour $M=N)$, on en conclut qu'il existe un nombre $\xi_{2}$ et un ensemble $Y$ satisfaisant-aux conditions:

$$
\begin{gathered}
\xi_{1}<\omega_{c f(\alpha)} \text { et } A_{\xi_{1}} \cdot X=A_{\xi_{q}} \cdot Y, \\
Y_{\in} N .
\end{gathered}
$$

Il résulte aussitôt de (15) que

$$
A_{\xi_{1}} \cdot X=A_{\xi_{1}} \cdot A_{\xi_{1}} \cdot X=A_{\xi_{1}} \cdot A_{\xi_{1}} \cdot Y .
$$

Si l'on avait $\xi_{2}<\xi_{1}<\omega_{c f(\alpha)}$, on obtiendrait de (3): $A_{\xi_{4}} \subset A_{\xi_{1}}$, d'où, en vertu de (17), $A_{\xi_{1}} \cdot X=A_{\xi_{1}} \cdot X, A_{\xi_{1}} \cdot X-A_{\xi_{1}} \cdot X=0$, ce qui contredit évidemment à (14). On a par conséquent, en raison de (14) et (15), $\xi_{1} \leqslant \xi_{2}<\omega_{c f(\alpha)}$, d'où suivant (3) $A_{\xi_{1}} \subset A_{\xi_{5}}$; cette inclusion, rapprochée de (17), donne: $A_{\xi_{1}} \cdot X=A_{\xi_{1}} \cdot Y$. On en obtient ensuite: $A_{\xi} \cdot A_{\xi_{1}} \cdot X=A_{\xi} \cdot A_{\xi_{1}} \cdot Y$; comme en outre, selon (3) et (14), $A_{\xi} \subset A_{\xi}$, on a finalement

$$
A_{\xi} \cdot X=A_{\xi} \cdot Y .
$$

Il est ainsi prouvé que pour tout nombre $\xi$ vérifiant l'inégalité (13) il existe un ensemble $Y$ remplissant les formules (16) et (18). A l'aide de l'axiome du choix on peut done faire correspondre d'une facon univoque a tout $\xi<\omega_{c f(\alpha)}$ un ensemble $Y$ assujetti à ces conditions; en d'autres termes, il existe une suite d'ensembles $B_{\xi}\left(\right.$ du type $\left.\omega_{c f(\alpha)}\right)$ vérifiant les formules suivantes:

$$
\begin{gathered}
A_{\xi} \cdot X=A_{\xi} \cdot B_{\xi} \text { pour } \xi<\omega_{c f(\alpha)} \\
B_{\xi} \in N, \text { lorsque } \xi<\omega_{c f(\alpha) .}
\end{gathered}
$$

Toute suite d'ensembles croissants est, comme on sait, nne suite convergente dans le sens de la déf. $7^{\circ}$, et sa limite est égale à l'ensemble-somme de tous ses termes. Cela concerne, en particulier, des suites $A_{\xi}$ et $A_{\xi} \cdot B_{\xi}$ du type $\omega_{\text {ef( }(\alpha)}$, qui en raison de (3) et (19) sont des suites d'ensembles croissants. D'après (1), (12) et (19), on a donc

$$
\lim _{\xi<\omega_{\text {cff }(\alpha)}} A_{\xi}=1 . \lim _{\xi<\omega_{\text {ff( }(\alpha)}}\left(A_{\xi} \cdot B_{\xi}\right)=X \text {. }
$$

En posant dans le lem. $12^{\mathrm{b}}: F_{\xi}=B_{\xi}$ et $G_{\xi}=A_{\xi}$ (et en $\mathrm{y}$ remplaçant $\alpha$ par $\left.\omega_{c f(\alpha)}\right)$, on conclut de (21) que la suite d'ensembles $B_{\xi} \cdot \lim _{\xi<\omega_{c f(\alpha)}} A_{\xi}$ est également convergente et qu'on a $\lim _{\xi<\omega_{c f(\alpha)}}\left(B_{\xi} \cdot \lim _{\xi<\omega_{c f}(\alpha)} A_{\xi}\right)=$

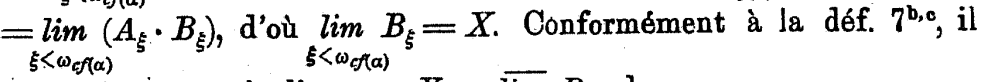

s'en suit en particulier que $X=\overline{\lim }_{\xi<\omega_{c f a}} B_{\xi}$, donc que

$$
X=\prod_{\xi<\omega_{c f(\alpha)}} \sum_{\xi \leqslant \eta<\omega_{c f(\alpha)}} B_{\eta} \text {. }
$$

En vertu de $(20)$ on a $\underset{B \eta}{E}\left[\xi \leqslant \eta<\omega_{c f(a)}\right] \subset N$; à cause de l'inégalité : $\beta>c f(\alpha)$, donnée par l'hypothèse, on obtient de plus sans 
peine: $\overline{B_{\eta}} \overline{\left[\xi \leqslant \eta<\omega_{c f(\alpha)]}\right.} \leqslant \kappa_{c f(\alpha)}<\aleph_{\beta}$. A l'aide de la déf. $2^{\text {b }}$ on en conclut que la classe $\underset{B_{\eta}}{E}\left[\xi \leqslant \eta<\omega_{c \mathcal{C}(\alpha)}\right]$ appartient à la famille $\boldsymbol{U}_{\beta}(N)$ et mềme à la famille $U_{\beta}(N)-\{0\}$ dans le cas où $\xi<\omega_{c f(\alpha)}$. Par conséquent, $\sum_{\xi \leqslant \eta\left\langle\omega_{c f f}(\alpha)\right.} B_{\eta}=\Sigma\left(\underset{B_{\eta}}{E}\left[\xi \leqslant \eta<\omega_{c f(\alpha)}\right]\right) \epsilon \bar{\Sigma}\left(\boldsymbol{U}_{\beta}(\boldsymbol{N})-\{0\}\right)$, donc en raison de la déf. 20

$$
\sum_{\xi \leqslant \eta\left\langle\omega_{c /(\alpha)}\right.} B_{\eta,} \in \boldsymbol{S}_{\beta}(\boldsymbol{N}) \text { pour } \xi<\omega_{c /(\alpha)}
$$

Or, en vertu de (7) et du th. $21^{\text {b }}$ on a $\boldsymbol{N}_{\in} \mathcal{C} \mathcal{P}\left(\boldsymbol{S}_{\beta}\right)$, d'où suivant la déf. $16 \boldsymbol{S}_{\beta}(\boldsymbol{N}) \subset \boldsymbol{N}$; en rapprochant cette inclusion de (23), on en déduit:

$$
\sum_{\xi \leqslant \eta<\omega_{\text {ef(a) }}} B_{\eta} \in N \text { pour. } \xi<\omega_{c f(a)}
$$

Par un raisonnement analogue (à cette différence près qu'au lieu de (20) et de la déf. 20 on a recours à la formule (24) et au th. 53) on parvient à la formule: $\prod_{\xi<\omega_{c f(a)}} \sum_{\xi \leqslant \eta\left\langle\omega_{c f(a)}\right.} B_{\eta \eta} \in \boldsymbol{N}$, qui donne

$$
X \in N
$$

La formule (26) est ainsi établie dans l'hypothèse (11). Or, d'après (10), cette formule se présente aussi dans l'hypothèse contraire; par conséquent, elle est généralement remplie.

Il est donc prouvé que la formule (9) entraîne (26), quel que soit l'ensemble $X$; ce fait peut etre exprimé tout court par l'inclusion: $M C N$. Comme l'inclusion inverse se laisse établir d'une façon
complètement analogue, on a finalement

$$
\boldsymbol{M}=\boldsymbol{N}
$$

Nous avons ainsi démontré que tontes deux classes $M$. et $\boldsymbol{N}$ qui remplissent les formules (7) et (8) vérifient également lidentité (27). Ce résultat peut être évidemment resumé dans la proposition suivante: $\boldsymbol{Y}$ étant une classe quelconque, si $\boldsymbol{M} \in \mathcal{E} \mathcal{P}\left(\boldsymbol{S}_{\beta}+\boldsymbol{S}_{\gamma}^{*}\right)$. $\underset{\boldsymbol{X}}{E}[\boldsymbol{F}(\boldsymbol{X})=\boldsymbol{Y}], \boldsymbol{N}_{\epsilon} \mathcal{Q} \mathcal{C}\left(\boldsymbol{S}_{\beta}+\boldsymbol{S}_{\gamma}^{*}\right) \cdot \underset{\boldsymbol{X}}{\boldsymbol{E}}[\boldsymbol{F}(\boldsymbol{X})=\boldsymbol{X}]$ et $\boldsymbol{G}(\boldsymbol{M})=$ $=G(N)$, on a $M=N$.
Par conséquent, la fonction $G$ transforme d'une façon biunivoque la famille $\mathcal{C}\left(\boldsymbol{S}_{\beta}+\boldsymbol{S}_{\gamma}^{*}\right) \cdot \underset{\boldsymbol{X}}{E}[\boldsymbol{F}(\boldsymbol{X})=\boldsymbol{Y})$ en son image $\overline{\boldsymbol{C}}\left(\mathscr{C}\left(\boldsymbol{S}_{\beta} \dot{\dagger} \boldsymbol{S}_{\gamma}^{*}\right)\right.$. $\cdot \underset{\boldsymbol{X}}{E}[\boldsymbol{F}(\boldsymbol{X})=\boldsymbol{Y}])$, de sorte que

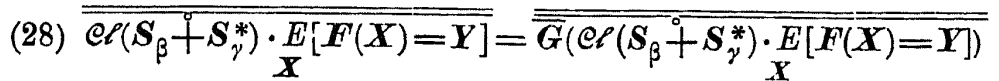

Remarquons qu'en vertu de (6) on a $G(\boldsymbol{M}) \subset \sum_{\xi<\omega_{\text {eff }}(a)} \boldsymbol{U}\left(\boldsymbol{A}_{\xi}\right)$ pour toute classe $M$, done $\bar{G}(\mathscr{R}) \subset U\left(\sum_{\xi<\omega_{c f(\alpha)}} \boldsymbol{U}\left(A_{\xi}\right)\right)$ pour toute famille de classes $\mathscr{H}$ et, en particulier,

$$
\overline{\boldsymbol{G}}\left(\mathcal{C P}\left(\boldsymbol{S}_{\beta}+\boldsymbol{S}_{\gamma}^{*}\right) \cdot \underset{\boldsymbol{X}}{\boldsymbol{E}}[\boldsymbol{F}(\boldsymbol{X})=\boldsymbol{Y}]\right) \subset \boldsymbol{U}\left(\sum_{\xi<\omega_{\mathcal{E}(\alpha)}} \boldsymbol{U}\left(A_{\xi}\right)\right) .
$$

Or, le lem. 10 donne: $\overline{\overline{U\left(A_{\xi}\right)}} \leqslant 2^{\overline{\bar{\pi}_{\xi}}}$, d'où en raison de (4) et de la déf. $4 \overline{\overline{U\left(A_{\xi}\right)}} \leqslant 2^{N_{a}}$ pour tout $\xi<\omega_{c f(\alpha)}$ et, par conséquent,

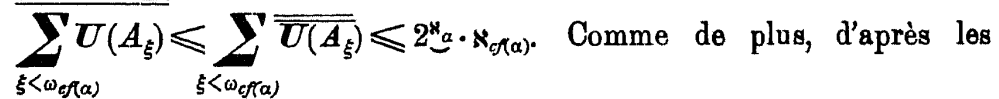

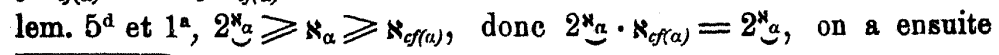
$\overline{\overline{\sum U\left(A_{\xi}\right)}} \leqslant 2^{x_{\alpha}}$. En appliquant une fois encore le lem. $10^{\mathrm{a}}$, nous $s<\omega_{e f f(a)}$

en obtenons:

$$
\overline{\overline{U\left(\sum_{\xi<\omega_{f f(\alpha)}} U\left(A_{\xi}\right)\right)}} \leqslant 2^{2^{*} \alpha .}
$$

On déduit facilement de (28)-(30) l'inégalité:

$$
\overline{\overline{\mathcal{Q}}\left(\boldsymbol{S}_{\beta}+-S_{\gamma}^{*}\right) \cdot \frac{E}{\boldsymbol{X}}[\boldsymbol{F}(\boldsymbol{X})=\boldsymbol{Y}]} \leqslant 2^{2^{\alpha_{\alpha}} .}
$$

En posant dans le lem. $11^{\mathrm{b}}: f=F$ et $A=\mathscr{C}\left(S_{\beta}+S_{\gamma}^{*}\right)$, on conclut de (31) que

$$
\overline{\overline{\mathcal{Q}\left(\boldsymbol{S}_{\beta}+\boldsymbol{S}_{\gamma}^{*}\right)}} \leqslant \overline{\left.\overline{\overline{\boldsymbol{F}}\left(\mathcal{Q} \ell\left(\boldsymbol{S}_{\beta}+\boldsymbol{S}_{\gamma}^{*}\right)\right.}\right)} \cdot 2^{2^{\boldsymbol{*}_{\alpha}}} .
$$

Il résulte d'autre part de (5) que $F(M) \subset \sum_{\xi<\omega_{C \mathcal{A}(\alpha)}} U\left(A_{\xi}\right)$ pour 
toute classe $M$, done que $\bar{F}(\mathscr{H}) \subset U\left(\sum_{\xi \in \omega_{c \mathcal{K}(a)}} U\left(A_{\mathfrak{G})}\right)\right)$ pour toute famille 2r, d'où, en particulier,

$$
\overline{\boldsymbol{F}}\left(\mathcal{E} \mathcal{C}\left(\boldsymbol{S}_{\beta}+\boldsymbol{S}_{\gamma}^{*}\right)\right) \subset \boldsymbol{U}\left(\sum_{\left.\varepsilon<\omega_{\mathcal{E f}(\alpha)} \boldsymbol{U}\left(A_{\xi}\right)\right)}\right.
$$

Les formules (30) et (33) entraînent aussitôt: $\overline{\overline{\overline{\boldsymbol{F}}\left(\mathcal{C} \mathcal{C}\left(\boldsymbol{S}_{\beta}+\boldsymbol{S}_{\gamma}^{*}\right)\right.}} \leqslant$

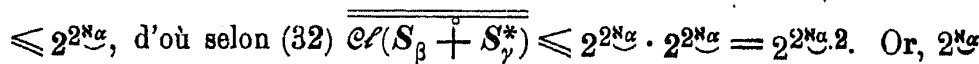
étant un nombre transfini (en vertu du lem. $5^{\mathrm{d}}$ ), on a $2^{\mathrm{N} \alpha} \cdot 2=2^{\mathrm{N} \alpha}$ et, par conséquent,

$$
\overline{\overline{\mathcal{Q} \mathcal{Q}\left(\boldsymbol{S}_{\beta}+S_{\gamma}^{*}\right)}} \leqslant 2^{2 * \alpha .}
$$

En rapprochant (1) de (34), on obtient enfin la formule cherchée:

$$
2^{* \alpha} \leqslant \overline{\overline{\mathcal{C}_{2}\left(\boldsymbol{S}_{\beta}+\boldsymbol{S}_{\gamma}^{*}\right)}} \leqslant 2^{2{ }^{2 \alpha} \alpha} .
$$

Thérème 70. Si $1=\aleph_{a}$ et $\beta>c f(a)$, on $a$

a) $2^{\mathrm{N}_{\alpha}} \leqslant \overline{\overline{\mathcal{Q P}\left(\boldsymbol{C}+\boldsymbol{S}_{\beta}\right)}} \leqslant 2^{2^{\mathrm{N}}{ }_{\alpha}}$;

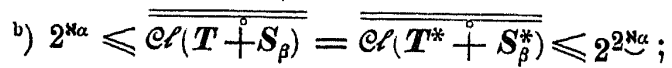

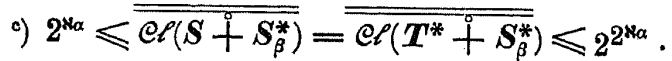

Démonstration. D'après le th. $51^{\mathrm{b}}$ et le lem. $19^{\mathrm{c}}$ on a:

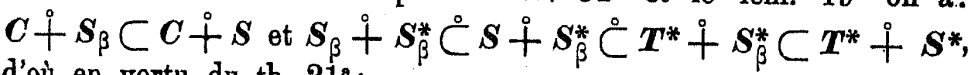
d'où en vertu du th. $21^{a}$.

$$
\mathfrak{Q} \mathcal{C}(\boldsymbol{C} \boldsymbol{S}) \subset \mathcal{e} \mathcal{C}\left(\boldsymbol{C}+\boldsymbol{s}_{\beta}\right)
$$

(2) $\mathscr{e}\left(\boldsymbol{T}^{*}+\boldsymbol{S}^{*}\right) \subset \mathcal{e}\left(\boldsymbol{T}^{*}+\boldsymbol{S}_{\beta}^{*}\right) \subset \mathcal{e}\left(\boldsymbol{S}+\boldsymbol{S}_{\mathrm{\beta}}^{*}\right) \subset \mathcal{Q}\left(\boldsymbol{S}_{\beta}+\boldsymbol{S}_{\beta}^{*}\right)$.

A cause du th. $50^{\mathrm{a}}$ on peut appliquer le th. $28^{\mathrm{b}}$, en y posant: $\boldsymbol{F}=S_{\beta} ;$ on tire: $\mathcal{C}\left(C+\boldsymbol{S}_{\beta}\right)=\mathcal{Q} \mathcal{C}\left(\boldsymbol{C}+\boldsymbol{S}_{\beta}+\boldsymbol{S}_{\beta}^{*}\right)$, done en raison du th. $21^{\text {b }}$ (pour $\boldsymbol{F}=C$ et $\left.G=S_{\beta}+S_{\beta}^{*}\right): \mathcal{Q} \mathcal{L}\left(C+S_{\beta}\right)=$ $=\mathscr{e}(\boldsymbol{C}) \cdot \mathcal{C}\left(\boldsymbol{S}_{\beta}+\boldsymbol{S}_{\beta}^{*}\right)$. Par conséquent,

$$
\mathfrak{e}\left(\boldsymbol{C}+\boldsymbol{S}_{\beta}\right) \subset \mathfrak{e} \mathcal{P}\left(\boldsymbol{s}_{\beta}+\boldsymbol{s}_{\beta}^{*}\right)
$$

A l'aide des th. fond. V et VII on déduit facilement de (1) et (2) les inégalités suivantes:

(4) $2^{*_{\alpha}} \leqslant \overline{\overline{\mathcal{Q} \mathcal{\ell}\left(\boldsymbol{C}+\boldsymbol{S}_{\beta}\right)}}$ et $2^{* \alpha} \leqslant \overline{\overline{\mathcal{Q} \mathcal{\ell}\left(\boldsymbol{T}^{*}+\boldsymbol{S}_{\beta}^{*}\right)}} \leqslant \overline{\overline{\mathcal{Q} \mathcal{Q}\left(\boldsymbol{S}+\boldsymbol{S}_{\beta}^{*}\right)}}$

De même, en raison du th. 69 (pour $\gamma=\beta$ ), les inclusions (2) et (3) donnent:

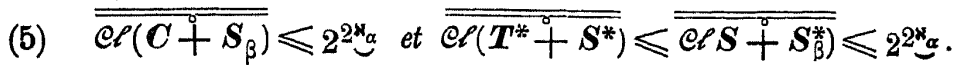

Il résulte du lem. $19^{\mathrm{a}, \mathrm{b}}$ que $\left[\boldsymbol{T}+\boldsymbol{S}_{\beta}\right]^{*}=\boldsymbol{T}^{*}+\boldsymbol{S}_{\beta}^{*}$ et que $\left[\boldsymbol{S}+\boldsymbol{S}_{\beta}^{*}\right]^{*}$ e. $\boldsymbol{S}^{*}+\boldsymbol{S}_{\beta} ;$ en appliquant donc à deux reprises lo th. 33 , on conclut enfin que

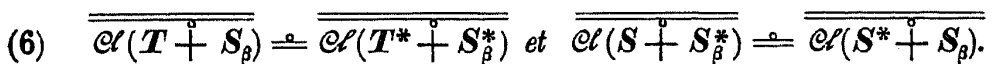
De $(4)-(6)$ on obtient immédiatement toutes les formules cherchées.

Théorème 71. Si $\overline{\overline{1}}=\aleph_{\alpha}$ et $\beta>c f(\alpha)$, on a

a) $2^{* \alpha} \leqslant \overline{\overline{\mathcal{Q} P\left(\boldsymbol{R}_{\beta}\right)}} \leqslant 2^{2{ }^{*} \alpha} ;$

b) $2^{\mathrm{*} \alpha} \leqslant \overline{\overline{\mathcal{Q} \ell\left(\boldsymbol{C}+\boldsymbol{R}_{\beta}\right)}} \leqslant 2^{2{ }^{*} \alpha}$

c) $2^{\mathrm{N}_{\alpha}} \leqslant \overline{\overline{\mathcal{Q} \mathcal{C}\left(\boldsymbol{T}+\overline{\boldsymbol{R}_{\beta}}\right)}}=\overline{\overline{\mathcal{Q} \mathcal{P}\left(\boldsymbol{T}^{*}+\boldsymbol{R}_{\beta}\right)}} \leqslant 2^{2^{\mathrm{N}_{\alpha}}}$

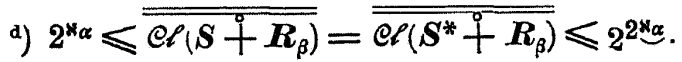

Démonstration est en grands traits analogue à celle du théorème précédent. A l'aide des th $55^{\mathrm{d}}, 51^{\mathrm{b}}, 42^{\mathrm{ab}}$ et des lemmes du \& 2 on établit sans peine les inclusions: $\boldsymbol{S}_{\beta}+\boldsymbol{S}_{\beta}^{*} \check{\complement}^{\circ} \boldsymbol{R}_{\beta} \check{\complement}^{\circ} \boldsymbol{S}^{*}+$

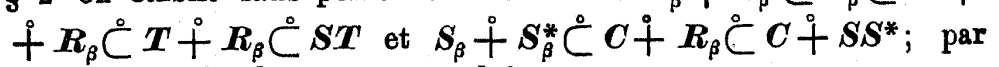
l'application du th. $21^{\mathrm{a}}$ on en obtient:

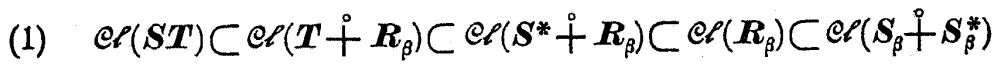

et

(2)

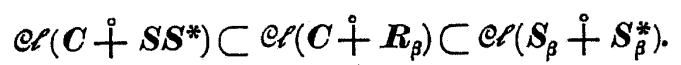

D'après les th. $24^{\mathrm{a}}, 42^{\mathrm{a}, \mathrm{b}}, 36^{\mathrm{b}}$ et le lem. 191 , on a $\mathcal{C}(\boldsymbol{S T})=$ $=\mathcal{C} t(\boldsymbol{S}+\boldsymbol{T})$ et $\mathcal{C} \mathcal{L}\left(\boldsymbol{S S}^{*}\right)=\mathcal{Q} \mathcal{L}\left(\boldsymbol{S}+\boldsymbol{S}^{*}\right)$; en raison du cor. 22 et du th. $28^{b}$, cette dernière formule entraîne: $\mathfrak{C}\left(\boldsymbol{C}+\boldsymbol{S S}^{*}\right)=$ 
$=\mathfrak{e}\left(\boldsymbol{C}+\boldsymbol{S}^{+} \boldsymbol{S}^{*}\right)=\mathcal{E} \mathcal{P}(\boldsymbol{C}+\boldsymbol{S})$. A l'aide des th. V et VII nous en concluons que

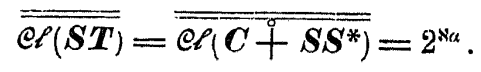

Suivant le th. 69 on a en outre:

$$
\overline{\mathcal{Q} \ell\left(S_{\beta}+S_{\beta}^{*}\right)} \leqslant 2^{2 N \alpha} \text {. }
$$

Les formules (1)-(4) donnent tout de suite:

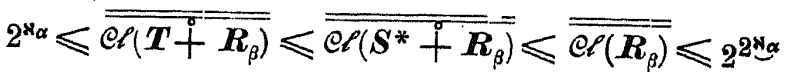

$$
\begin{aligned}
& 2^{\mathrm{N}_{\alpha}} \leqslant \overline{\overline{\mathcal{Q} \mathcal{C}\left(\boldsymbol{C}+\overline{+} \overline{\boldsymbol{R}_{\beta}}\right)}} \leqslant 2^{2^{\mathrm{N}_{\alpha}}} .
\end{aligned}
$$

Tenant compte du th. $55^{\mathrm{e}}$ et appliquant le th. 33 et le lem. 19d, on parvient ensuite aux égalités:

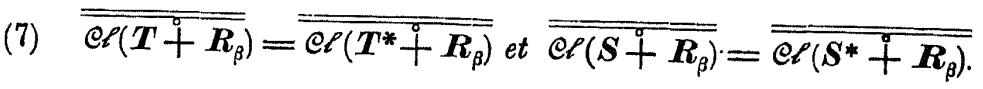

En rapprochant (7) de (5) et (6), on obtient les formules cherchées.

Nous avons examiné dans les th. IX-XI et $69-71$ la famille $\mathcal{C}\left(\boldsymbol{T}+\boldsymbol{S}_{\beta}\right)$ et les antres familles analogues dans les deux cas suivants: $\beta \leqslant p(a)$ et $\beta>c f(a)$ ( $\aleph_{a}$ étant la puissance de l'ensemble 1 ). Par contre, ces résultats n'embrassent pas le cas où $p(a)<\beta \leqslant c f(a)$; dans ce der cas nous no savons ni évaluer les puissances des familles conidérées, ni même établir pour elles des déliminations qui soient con-

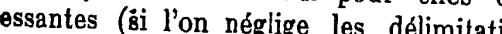
dn th. 49: $2^{\mathrm{N}_{\alpha}} \overline{\overline{\mathcal{Q P ( T + T )}}}$ dn th. 49: $\left.2^{\mathrm{N \alpha} \alpha} \leqslant \mathcal{Q} \mathcal{Q}\left(\boldsymbol{T}+\boldsymbol{S}_{\beta}\right) \leqslant 2^{\left.2^{\mathrm{N \alpha}}\right)}\right)$. En particulier, il n'est pas élucidé jusqu'à présent, si l'on peut généraliser les th. $69-71$, en y remplaçant pouna problème ne nous semble pas dépourva des chances. Cette lacune dans les résultats acquis jusqu'à présent ne se laisse combler qu'à l'aide des raisonnements faisant appel à l'hypothèse $\mathrm{H}$, étant donné que (comme nous l'avons déjà montré par le lem. $9^{b}$ ) cette hypothèse exclut le cas où $p(\alpha)<\beta \leqslant c f(\alpha)$.

Théorème fondamental XII. L'hypothèse $H$ entraîne les conséquences suivantes:
Si $\overline{\overline{1}}=\aleph_{\alpha}$ et $\beta>p(a)$, on $a$
2) $\overline{\overline{\mathcal{Q}}\left(\boldsymbol{C}+\overline{S_{\beta}}\right)}=2^{\boldsymbol{N}_{\alpha}}$;
b) $\overline{\overline{\mathcal{Q} P\left(\boldsymbol{T}+\overline{S_{\beta}}\right.}}=\overline{\overline{\mathcal{Q P}\left(\boldsymbol{T}^{*}+\boldsymbol{S}_{\beta}^{*}\right)}}=2^{\mathrm{N} \alpha_{\alpha}}$
c) $\overline{\overline{\mathcal{Q}\left(S+\boldsymbol{S}_{\beta}^{*}\right)}}=\overline{\overline{\mathcal{Q P}\left(\boldsymbol{S}^{*}+\boldsymbol{S}_{\beta}\right)}}=2^{* \alpha}$;
d) si en outre $\gamma>p(a)$, alors $\overline{\mathcal{Q} P\left(\boldsymbol{S}_{\beta}+\boldsymbol{S}_{\gamma}^{*}\right)}=2^{\mathrm{s} \alpha}$.

Démonstration. Il suffit de rappeler qu'en conséquence de l'hypothèse $\mathrm{H}$ on a les formules: $c f(a)=p(a)$ et $2^{* \alpha}=\kappa_{\alpha}\left(\right.$ lem. $\left.9^{\mathrm{a}, \mathrm{b}}\right)$, et d'appliquer ensuite les th. 69 et 70 .

D'une façon analogue on déduit du th. 71 le

Théorème 72. L'hypothèse $H$ entraîne la conséquence suivante:

$$
\begin{aligned}
& \text { Si } \overline{\bar{I}}=\kappa_{\alpha} \text { et } \beta>p(a) \text {, on a } \overline{\overline{\mathcal{Q} \ell\left(\boldsymbol{R}_{\beta}\right)}}=\overline{\overline{\mathcal{Q} \ell\left(\boldsymbol{C}+\boldsymbol{R}_{\beta}\right)}}=\overline{\overline{\mathcal{Q}_{\boldsymbol{\gamma}}\left(\boldsymbol{T}+\boldsymbol{R}_{\beta}\right)}}=
\end{aligned}
$$

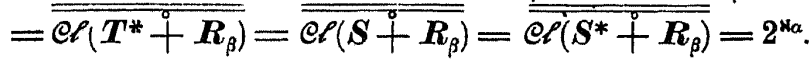

Il est intéressant d'examiner les difficultés auxquelles doivent se heurter nécessairement toutes les tentatives d'éviter l'hypothèse $H$ dans les démonstrations des th. XII et 72.

Supposons, en effet, que nous réussîmes de démontrer une de ces formules, p. ex. le th. XII ${ }^{\mathrm{b}}$, sans cetle hypothèse. Nous obtenons comme cas particulier (pour $\alpha=\omega$ et $\beta=1$ ) la proposition suivante:

$$
\text { A. } S i \overline{\overline{1}}=\aleph_{\omega} \text {, on } a \overline{\overline{\mathcal{Q P}\left(T+\boldsymbol{T}_{1}\right)}}=2^{\mathrm{*}_{\omega}} \text {. }
$$

Admettons ensuite que l'hypothèse ordinaire du contina: $2^{\mathrm{x}_{4}}=\mathrm{K}_{\mathrm{K}}$ soit vraie. En vertu du théorème connu de M. Bernstein ${ }^{1}$, nous aurons alors pour tout nombre ordinal $\alpha$ tel que $0<\alpha<\omega$ les égalités: $\aleph_{\alpha}^{\aleph_{0}}=\aleph_{\alpha} \cdot 2^{\aleph_{0}}=\aleph_{\alpha} \cdot \aleph_{1}=\aleph_{\alpha}$, donc, par suite da lem. $4^{c}, p(\alpha) \leqslant 1$. En po- sant: $\beta=1$ dans le th. $X$, on obtient par conséquent:

B. Si $2^{\aleph_{0}}=\aleph_{1}$ et $\overline{\bar{I}}=\aleph_{\alpha}$, où $0<\alpha<\omega$, on $a$ $\mathcal{P}\left(\boldsymbol{T}+\boldsymbol{S}_{1}\right)=2^{2^{\alpha_{\alpha}}}$.

La proposition $B$ pent être généralisée facilement comme il sait:

C. Si $2^{\aleph_{0}}=\aleph_{1}$ et $\overline{\bar{A}}=\aleph_{\alpha}$ ò̀ $0<\alpha<\omega$, on $a$

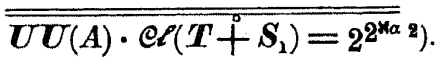

1) Untersuchungen aus der Mengenlehre (Inaugural-Dissertation), Hallo 8. 1901, p. 49 (ou Math. Ann. 61).

2) Au fond cette "généralisation" est illusoire, car la proposition $C$ ne constitue qu'un antre ́noncé de la proposition $B$. J'ai eu, en effet, occasion de moutionner ici plus d'une fois (p, 183, 212) que le symbole 1 joue dans cet onvrage 
Prenant pour 1 un ensemble arbitraire de la puissance $\aleph_{\omega}$ et considérant son sous-ensemble quelconque $A$ de la puissance $\aleph_{\alpha}$ où $\alpha<\omega$, on déduit des propositions $\mathrm{A}$ et $\mathrm{C}$ la conséquence:

D. Si $2^{\aleph_{0}}=\aleph_{1}$ et $\alpha<\omega$, on a $2^{2{ }^{* \alpha}} \leqslant 2^{N_{\omega}}$ et $2^{N_{\alpha}}<2^{N_{\omega}}$.

Or, toutes les tentatives d'établir à l'aide de l'hypothèse ordinaire du contina les inégalités semblables à celles qui constituent la thèse de la proposition $D$ se sont terminées jusqu'à présent par un échec complet:

On doit donc considérer comme peu probable que la proposition A et, par conséquent, le th. XII ${ }^{b}$ soient démontrables sans l'hypothèse du continu généralisée.

J'ai signalé dans mes notes antérieures ${ }^{1}$ ) que certains résultats acquis à l'aide de l'hypothèse $H$ se laissent établir sans elle, à condition toutefois de restreindre le domaine des nombres cardinaux considérés à un système, d'ailleurs assez vaste, des nombres $\aleph_{\pi(\alpha)}$ définies par recurrence comme suit:

$$
\begin{gathered}
\aleph_{\pi(0)}=\aleph_{0} ; \aleph_{\pi(\alpha)}=2^{\aleph_{\pi(\alpha-1)}} \text { pour } \alpha \text { de } 1^{r e} \text { espèce; } \\
\aleph_{\pi(\alpha)}=\sum_{\xi<\alpha} \aleph_{\pi(\xi)} \text { pour } \alpha \text { de } 2^{m e} \text { espèce, } \alpha \neq 0 .
\end{gathered}
$$

Or, la situation est encore la même en ce qui concerne les th. XII et 72: toutes les formules de ces théorèmes peuvent être établies sans l'hypothèse $H$ à condition que $\alpha$ soit de la forme $\alpha=\pi\left(\alpha_{1}\right)$, où $\alpha_{1}$ est un nombre de $2^{\mathrm{m \theta}}$ espèce. En effet, on peut montrer alors que $p(\alpha)=$ $=c f(\alpha)=c f\left(\alpha_{1}\right)$ et que $\left.2^{\aleph_{\alpha}}=\aleph_{\alpha}^{2}\right) ;$ or, ce sont les seules conséquences de l'hypothèse $\mathrm{H}$ qui interviennent dans la démonstration da th. XII.

Observons encore que, comme nous l'avons montré dans le cor. 63 , toutes les familles de la forme $\mathcal{C} \mathcal{P}(\boldsymbol{F})$ où $\boldsymbol{F}$ est une opération assujettio à l'inclusion: $\boldsymbol{F} \subset \boldsymbol{C} \boldsymbol{S}_{\beta}$, donc en particulier une opération semi-additive an dégré $\beta$ et intrinsèque, ont la même puissance, à savoir $2^{2^{* \alpha}}$, dans l'hypothèse que $\beta \leqslant p(\alpha)$ ( $\kappa_{\alpha}$ étant la puissance de 1 ). Par contre, dans

le rôle d'un signe variuble dénotant l'ensemble de tous les individus considérés. dans un théorème donné.

Il est pent-être superflu d'ajouter que de la même façon qui vient d'être employée pour "généraliser" la proposition B se laissent "généraliser" toùs les théorèmes de ce mémoire concernant la puissance des fanilles de la forme $\mathcal{Q} P\left(\boldsymbol{F}^{\prime}\right)$
où $F$ est une opération intrinsèque quelconqne.

1) Quelques théorèmes sur les alephs, Fund. Math. VII, p. 9-10; Sur la décomposition des ensembles en sous-ensembles presque disjoints, Fond. Math. XII, p. 204, et Fund. Math. XIV, p. 213.

2) La première de ces formules résulte du th. II, énoncé dans ma note précitée de Fund. Math. VII, p. 9; la seconde présente une conséquence facile de la définition des nombres $\aleph_{\pi(\alpha)}$. le cas de $\beta>p(\alpha)$ les familles du type considéré n'ont pas les puissances égales. On peut indiquer, en effet, comme exemples des opérations $F$ semi-additives au dégré $\beta$ et intrinsèques les opérations $T, S_{\beta}$ et $S^{*}$ d'ane part et les opérations $\boldsymbol{T}+\boldsymbol{S}_{\beta}, \boldsymbol{S}_{\beta}+\boldsymbol{S}_{\beta}^{*}, \boldsymbol{R}_{\beta}$ et $\boldsymbol{T}+\boldsymbol{R}_{\beta}$ d'autre part; or, on aura dans le premier cas $\overline{\overline{\mathcal{C} P\left(F^{r}\right)}}=2^{2^{\text {sa }}}$ (th. II, VIII) et dans le second $\overline{\overline{\mathcal{C} P\left(\boldsymbol{F}^{\prime}\right)}}=2^{\text {sa }}$ (th. XII, 72).

Pour terminer il y a lieu de dire quelques mots sur certaines opérations qui viennent se lier à celles examinées dans ce §. C'est une généralisation de la déf. 20 qui est à mentionner arant tout. Introduisons notamment l'opération $\boldsymbol{S}_{(\mathfrak{b})}$ définie par la formule: $\boldsymbol{S}_{(\mathfrak{b})}(\boldsymbol{K})=E[\boldsymbol{X} \subset \boldsymbol{K}$ et $0<\overline{\bar{X}}<b]^{1}$ ). Si b est un nombre transfini, à savoir $\mathbf{b}=\boldsymbol{\aleph}_{\beta}$, l'opération $\boldsymbol{S}_{(\mathfrak{b})}$ coïncide avec $\boldsymbol{S}_{\beta}$; par contre, dans le cas de $\mathfrak{b}$ fini nous sommes en présence d'une opération nouvelle. Cependant cette généralisation est dépourvue de valeur an point de rae de ces considérations: car pour $\mathfrak{b} \leqslant 2$ l'opération $S_{(\mathfrak{b})}$ est banale $\left(\boldsymbol{S}_{(0)}(\boldsymbol{K})=\boldsymbol{S}_{(1)}(\boldsymbol{K})=0\right.$ pour tonte classe $\left.\boldsymbol{K}, \boldsymbol{S}_{(2)}=\boldsymbol{I}\right)$; si, par contre, $2<\mathbf{b}<\boldsymbol{s}_{0}$, on constate facilement que $\mathcal{Q} \mathcal{P}\left(\boldsymbol{S}_{(\mathfrak{b})}\right)=\mathcal{Q} \mathcal{\ell}\left(\boldsymbol{S}_{0}\right)$.

Ensuite c'est l'operation $\boldsymbol{B}_{\beta, \gamma}$, définie par la formule: $\boldsymbol{B}_{\beta, \gamma}(\boldsymbol{K})=$ $=\mathbb{H}\left(\boldsymbol{V}(\boldsymbol{K}) \cdot \mathfrak{C} \mathcal{l}\left(\boldsymbol{S}_{\beta}+\boldsymbol{S}_{\gamma}^{*}\right)\right)$, qui mérite l'attention. La classe $\boldsymbol{B}_{\beta, \gamma}(\boldsymbol{K})$ est donc la plus petite classe contenant la classe $\boldsymbol{K}$ et close par rapport aux opérations $S_{\beta}$ et $\boldsymbol{S}_{\gamma}^{*}$ (cf. p. 272, 276); on pourrait l'appeler classe d'ensembles boreliens formés de la classe $\boldsymbol{K}$, additive au dégré $\beta$ et multiplicative au dégré $\gamma$. L'opération $\boldsymbol{B}_{\beta, \gamma}$ a élé éludiée jusqu'à présent surlout dans le cas où $\beta=\gamma=1$, où elle coïncide avec l'opération borelienne habituelle, et de plus dans le cas: $\beta=\gamma=0$ et $\beta=2, \gamma=1^{2}$ ). An point de vae de ces recherches cette opération est encore sans grande importance, à cause de la formule facile à établir: $\mathcal{C} \mathcal{P}\left(\boldsymbol{B}_{\beta, \gamma}\right)=\mathcal{Q} \mathcal{P}\left(\boldsymbol{S}_{\beta}+\boldsymbol{S}_{\gamma}^{*}\right)$.

\section{§ 7. Opération $\boldsymbol{D}$. Classes d'ensembles soustractives.}

L'opération $\boldsymbol{D}$ consiste à ajouter à la classe d'ensembles donnée $\boldsymbol{K}$ toutes les différences des ensembles appartenant à cètte classe; les classes d'ensembles closes par rapport à cette opération s'appellent classes soustractives.

Définition 22. $D(K)=K+\underset{X-Y}{E}\left[X \in K\right.$ et $\left.\boldsymbol{Y}_{\epsilon} \boldsymbol{K}\right]$.

Parmi les numbreuses propriétés élémentaires de l'opération $\boldsymbol{D}$ je ne cite ici que celles qui sernnt utilisées dans la suite.

1) Cf. l'article de MM. Koźniewski et Lindenbaum, cité ici p. 249, note 1). 2) Cf. p. 254, notes '), 2 ). 
Théorème 73. aj $D \in \mathfrak{Z}_{0}$, donc $\boldsymbol{D} \in \mathfrak{Z}_{\beta}$.et $\boldsymbol{D} \in \mathfrak{M}$;

b) $I \subset D$;

a) $\boldsymbol{D}(0)=0$

a) $\boldsymbol{D} \stackrel{\complement}{\boldsymbol{T}}$

o) $\boldsymbol{D} \dot{\complement} \boldsymbol{S}_{0}^{*}[\boldsymbol{I}+\boldsymbol{C}]$;

f) $S_{0}^{*} \subset \sum_{1 \leq v<\omega}^{\circ} D^{\nu}$

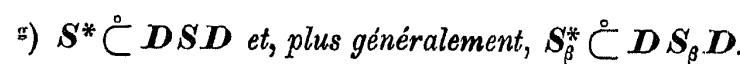

Démonstration. Les formules a)--d) résultent presque immédiatement de la déf. 22 et des définitions précédentes.

Pour établir la formule ${ }^{\circ}$ ), ubservons que tout ensemble de la classe donnée $K$ et, en vertu de la formule bien connue: $X-Y=$ $=X \cdot(1-Y)$, même toute différence de deux ensembles de cette classe se laissent représenter comme produit de deux, donc de moins què $\aleph_{0}$, ensembles de la classe $\boldsymbol{K}+\underset{1-V}{E}[Y \in \boldsymbol{K}]$. D'après la déf. 22 et le th. 53, on a donc $\boldsymbol{D}(\boldsymbol{K}) \subset \boldsymbol{S}_{0}^{\text {1-V }}\left(\boldsymbol{K}+\underset{,-Y}{E}\left[Y_{\epsilon} \boldsymbol{K}\right]\right)$, d'où on obtient aussitôt, à l'aide des définitions du $\S 2$, l'inclusion cherchée: $\boldsymbol{D} \stackrel{\complement}{\complement} \boldsymbol{S}_{0}^{*}[\boldsymbol{I} \stackrel{+}{C}]$.

f) La formule: $X \cdot Y=X-(X-Y)$ implique que $X \cdot Y \in \boldsymbol{D D}(\boldsymbol{K})$, à condition que $X_{\epsilon} \boldsymbol{K}$ et $Y_{\epsilon} \boldsymbol{K}$. En raison de la déf. $10^{\mathrm{b}, \mathrm{c}}$, on en conclut par une induction facile que $\prod_{1 \leqslant \xi \leqslant v+1} X_{\xi} D^{2 . \nu}(\boldsymbol{K})$, lorsque $\nu<\omega$ et $X_{\xi} \in \boldsymbol{K}$ pour $1 \leqslant \xi \leqslant \nu+1$. Or, par suite du th. 53 , ces ensembles $\prod_{1 \leqslant \xi \leqslant v+1} X_{\xi}$ forment la classe $\boldsymbol{S}_{0}^{*}(\boldsymbol{K}) ;$ on a donc $\boldsymbol{S}_{0}^{*}(\boldsymbol{K}) \subset$ $\subset \sum_{1 \leqslant \nu<\omega} D^{\nu}(K)$, d'où $S_{0}^{*} \check{C}_{1 \leqslant \nu<\omega}^{\circ} D^{\nu}$, c. q. f. d.

La formule s) s'obtient d'une façon analogue à la formule ${ }^{\circ}$ ), en

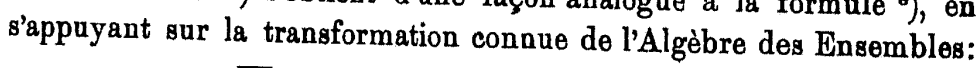
$\Pi(\boldsymbol{K})=X-\sum_{Y_{\epsilon} K}(X-Y)$ pour tout $X \in \mathcal{K}$, et en faisant appel à la déf. 18 et au th. 44, resp. (en ce qui concerne la deuxième inclusion) à la déf. 20 et au th. 53. Il est à remarquer que dans la démonstration de cette deuxième inclusion on a en outre recours an lem. 11a (pour prouver que $\overline{\bar{E}[\overline{[\overline{Y \in \boldsymbol{K}}]}} \leqslant \overline{\overline{\boldsymbol{K}}}$ ), done, indirectement, à l'axiome du choix.

L'opération $\boldsymbol{D}^{*}$, double de $\boldsymbol{D}$, est caractérisée par le théorème suivant, qui résulte facilement des déf. 14,15 et 22 :

Théorème 74. $D^{*}(\boldsymbol{K})=\boldsymbol{K}+\underset{(1-X)+Y}{E[X \in K}$ et $\left.Y_{\epsilon} \boldsymbol{K}\right]$.

Cette opération consiste donc à ajouter à une classe d'ensembles $\boldsymbol{K}$ des quotients des éléments de cette classe, en entendant par quotient des ensembles $Y$ et $X$ l'ensemble $Z=Y: X=(1-X)+Y$.

A cause du lem. 19 les propriétés de l'opération $D^{*}$ se déduisent immédiatement de celles qui leur correspondent pour l'opération $\boldsymbol{D}$ et qui ont été formulées dans le th. 73. On a en outre le suivant

Thérème 75. $C \check{C}^{\circ} D D^{*}$.

Démonstration. Considérons une classe quelconque $\boldsymbol{K}$. Soit $X_{\epsilon} \boldsymbol{C}(\boldsymbol{K})$, done $X=1-Y$ où $Y \in \boldsymbol{K}$ (déf. 14), d'où en raison du th. $74 Y_{\epsilon} D^{*}(\boldsymbol{K})$ et $1=(1-Y)+Y_{\epsilon} D^{*}(\boldsymbol{K})$; par conséquent, d'après la déf. 22, $X=1-Y \in \boldsymbol{D} \boldsymbol{D}^{*}(\boldsymbol{K})$. Il s'en suit que $\boldsymbol{C}(\boldsymbol{K}) \subset$ $C \boldsymbol{D} D^{*}(\boldsymbol{K})$ pour toute classe $\boldsymbol{K}$, done que $\boldsymbol{C} \complement^{\circ} \boldsymbol{D} \boldsymbol{D}^{*}$, c. q. f. d.

Je vais indiquer ici quelques propriélés des opérations $\boldsymbol{D}$ et $\boldsymbol{D}^{*}$, omises dans les théorèmes précédents. Le th. $73^{\mathrm{a}}$ peut être renforcé comme il suit: $\boldsymbol{D}(Y)=\boldsymbol{\sum} \boldsymbol{D}(X)$ pour toute classe d'ensembles $\boldsymbol{Y}$, $X \subset \boldsymbol{Y}$ et $\overline{\bar{X}}<s$

d'où, en particulier, $\boldsymbol{D}(\boldsymbol{X}+\boldsymbol{Y}+\boldsymbol{Z})=\boldsymbol{D}(\boldsymbol{X}+\boldsymbol{Y})+\boldsymbol{D}(\boldsymbol{X}+\boldsymbol{Z})+$ $+\boldsymbol{D}(\boldsymbol{Y}+\boldsymbol{Z})$ pour toutes classes $\boldsymbol{X}, \boldsymbol{Y}$ et $\boldsymbol{Z}$. On a ensuite les théorèmes: si $\boldsymbol{K} \neq 0$, alors $0 \in \boldsymbol{D}(\boldsymbol{K})$ et $1 \in \boldsymbol{D}^{*}(\boldsymbol{K})$; si $1 \in \boldsymbol{K}$, on a $\boldsymbol{C}(\boldsymbol{K}) \subset \boldsymbol{D}(\boldsymbol{K})$; si $0 \in \boldsymbol{K}$, on a $\boldsymbol{C}(\boldsymbol{K}) \subset \boldsymbol{D}^{*}(\boldsymbol{K}) ; \boldsymbol{D} \check{C}^{\circ} \boldsymbol{T C} ; \boldsymbol{D C} \check{C}^{\circ}$ $\mathscr{C}^{\circ} \boldsymbol{D}+\boldsymbol{C} ; \boldsymbol{C} \subset \boldsymbol{D}^{*} \boldsymbol{D}$ (la formule double de celle du th. 75);

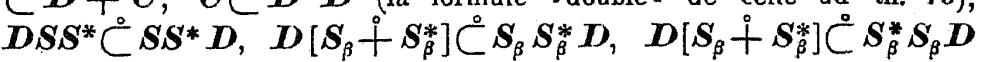

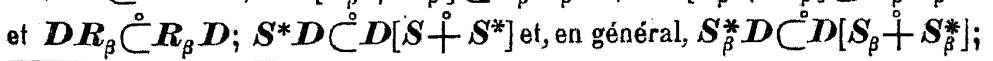
$\overline{\overline{\boldsymbol{D}(\overline{\boldsymbol{K}})}} \leqslant \overline{\overline{\boldsymbol{K}}}+(\overline{\overline{\boldsymbol{K}}})^{2}$. Il est enfin à noter que $\boldsymbol{M} \boldsymbol{D}(\boldsymbol{K})$ est une classe $\boldsymbol{d}^{\prime}$ ensembles disjoints, quelle que soit la classe $\boldsymbol{K}$. (c.. déf. 19).

Au lieu de l'opération $D$, on considére d'habitade l'opération $D_{1}$, déterminée par la formule: $\boldsymbol{D}_{1}(\boldsymbol{K})=\underset{X-Y}{E}\left[X_{\epsilon} \boldsymbol{K}\right.$ et $\left.Y_{\epsilon} \boldsymbol{K}\right]$, et l'on dé-

Fundamenta Mathomaticae. T. XVI. 
signe la classe $D_{1}(K)$ par $\left.K_{\rho}{ }^{1}\right)$. Les opérations $D$ et $D_{1}$ sont liẻes par les relations très étroites, p. ex.: si $0 \in \in$, on a $D(K)=D_{1}(K)$; $D D_{1} \cong D_{1}^{2}$ et $D_{1} D=D^{2} ; D=D_{1}+\boldsymbol{I}$. Cette dernière égalité donne: $\mathscr{C}(\boldsymbol{D})=\mathscr{C} \mathcal{C}\left(\boldsymbol{D}_{1}\right)$, de sorte qu'il est indifférent au point de vue de ces recherches, laquelle des deux opérations $D$ et $D_{1}$ en sera choisie pour l'objet (comp. le th. $23^{\mathrm{b}}$ et remarques qui l'accompagnent). Parmi les autres propriétés de $D_{1}$ citons enfin les denx suivantes: $D_{1} \rightleftharpoons D_{1} C$ et $D_{1}^{2} \rightleftharpoons D_{1} D_{1}^{*}$.

Je passe aux familles des classes closes par rapport aux opérations $\boldsymbol{D}$ et $\boldsymbol{D}^{*}$ et aux opérations de la forme $\boldsymbol{F}+\boldsymbol{D}, \boldsymbol{F}+\boldsymbol{D}^{*}$ et $\boldsymbol{F}+\boldsymbol{D}+D^{*}$, où $\boldsymbol{F}$ est une des opérations examinées dans les $\S \S$ précédents. Cette étude est considérablement simplifiée par certaines identités qui existent entre ces familles et que je vais établir au préalable.

Notons que les classes de la famille $\mathcal{E} \mathcal{C}\left(\boldsymbol{S}_{\beta} \stackrel{+}{+}\right)$ ont été étudiées déjá, bien qu'á un point de vue différent, par M. Ha u sdorff, qui les appelle Körper dans le cas où $\beta=0$ et erweiterte Körper dans le cas général 2).

Théorème 76. $\mathscr{C}(\boldsymbol{D}) \subset \mathcal{O}\left(\boldsymbol{S}_{0}^{*}\right), \mathscr{C}\left(\boldsymbol{D}^{*}\right) \subset \mathcal{O P}\left(\boldsymbol{S}_{0}\right)$.

Démonstration. Tenant compte du th. $73^{\mathrm{r}}$ et appliquant le th. $21^{\mathrm{a}}$, on obtient: $\mathcal{e}\left(\sum_{1 \leqslant v<\omega}^{\circ} D^{\nu}\right) \subset \mathcal{C} \mathscr{C}\left(S_{0}^{*}\right)$, d'où en raison du th. $21^{\mathrm{b}}$ :

$$
\prod_{1 \leq \boldsymbol{\nu}<\omega} \mathcal{C} \mathcal{P}\left(\boldsymbol{D}^{\boldsymbol{v}}\right) \cdot \subset \mathcal{O} \mathcal{C}\left(\boldsymbol{S}_{0}^{*}\right)
$$

$D^{\prime}$ autre part le th. 25 donne en vertu du th. $73^{\text {b }}$ :

$$
\mathscr{C}\left(\boldsymbol{D}^{\nu}\right)=\mathfrak{Q} \mathcal{P}(\boldsymbol{D}) \text { pour } 1 \leqslant \nu<\omega .
$$

Il résulte aussitôt de (1) et (2) quẹ

$$
\mathscr{e}(\boldsymbol{D}) \subset \mathcal{Q} P\left(\boldsymbol{S}_{0}^{*}\right)
$$

d'où $\bar{C}\left(\mathscr{e}\left(D_{j}\right) \subset \bar{C}\left(\mathscr{e}\left(S_{0}^{*}\right)\right)\right.$; à l'aide du th. 26 on en conclut que $\mathfrak{C}\left(D^{*}\right) \subset \mathfrak{C}\left(\boldsymbol{S}_{0}^{* *}\right)$, et on a donc d'après le lem. $19^{\mathrm{b}}$ :

$$
\mathcal{Q} \mathcal{P}\left(\boldsymbol{D}^{*}\right) \subset \mathcal{Q} \mathcal{P}\left(\boldsymbol{S}_{0}\right) \text {. }
$$

1) Cf. le livre de M. Sierpiński, p. 113, citè ici p. 254, note $\%$.

2) Cr. Hau sdorff, Mengenlehre, II Aufl., Berlin und Leipzig 1927, p. 78-82.
Les formules (3) et (4) constituent le théorème, qui se trouve ainsi démontré.

Théorème 77. a) $\mathfrak{C}(C+D)=\mathscr{C}\left(C+D^{*}\right)=\mathscr{C}\left(C+D \nmid D^{*}\right)=$ $=\mathscr{Q}\left(D+D^{*}\right)=\mathscr{Q} \mathcal{C}\left(\boldsymbol{C}+\boldsymbol{S}_{0}\right)$;

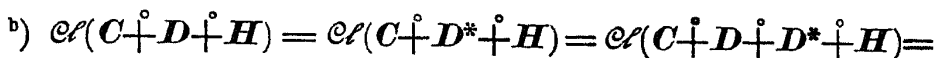
$=\mathfrak{C}\left(\boldsymbol{D}+\boldsymbol{D}^{*}+\boldsymbol{H}\right)=\mathscr{C}\left(\boldsymbol{C}+\boldsymbol{S}_{0}+\boldsymbol{H}\right)$ pour toute opération $\boldsymbol{H}$.

Démonstration. a) D'après les th. $28^{b}$ et $73^{a}$, on a

(1) $\quad \mathcal{Q}(\boldsymbol{C}+\boldsymbol{D})=\mathfrak{e}\left(\boldsymbol{C}+\boldsymbol{D}^{*}\right)=\mathfrak{e} l\left(\boldsymbol{C}+\boldsymbol{D}+\boldsymbol{D}^{*}\right)$.

Le th. $21^{b}$ (pour $\boldsymbol{F}=\boldsymbol{C}$ et $\left.\boldsymbol{G}=\boldsymbol{D}+\boldsymbol{D}^{*}\right)$ donne: $\mathcal{O P}(\boldsymbol{C} \dot{+}$ $\left.\stackrel{+}{\boldsymbol{D}} \stackrel{\circ}{+} \boldsymbol{D}^{*}\right)=\mathcal{C} \mathcal{L}(\boldsymbol{C}) \cdot \mathfrak{e}\left(\boldsymbol{D}+\boldsymbol{D}^{*}\right)$, d'où

$$
\mathfrak{e}\left(\boldsymbol{C}+\boldsymbol{D} \stackrel{\circ}{+} \boldsymbol{D}^{*}\right) \subset \mathcal{e}\left(\boldsymbol{D}+\boldsymbol{D}^{*}\right) \text {. }
$$

Comme $\boldsymbol{D}^{*} \mathcal{\complement}^{\mathrm{D}} \boldsymbol{D}+\boldsymbol{D}^{*}$, on conclut du th. $21^{\mathrm{a}}$ que $\mathcal{Q}\left(\boldsymbol{D}+\boldsymbol{D}^{*}\right) \subset$ $\mathcal{C} \mathcal{C}\left(\boldsymbol{D}^{*}\right)$, donc, en raison du th.' 76 ,

$$
\mathfrak{e}\left(\boldsymbol{D} \dot{+} \boldsymbol{D}^{*}\right) \subset \mathcal{e} \mathcal{P}\left(\boldsymbol{S}_{0}\right)
$$

Les inclusions: $\mathscr{e}\left(\boldsymbol{D}+\boldsymbol{D}^{*}\right) \subset \mathcal{e}\left(\boldsymbol{D} \boldsymbol{D}^{*}\right)$ et $\mathscr{e}\left(\boldsymbol{D} \boldsymbol{D}^{*}\right) \subset \mathcal{e} \mathcal{P}(\boldsymbol{C})$, dont la première résulte des th. $24^{\mathrm{a}}$ et $73^{\mathrm{a}}$ et la seconde se déduit des th. $21^{\mathrm{a}}$ et 75 , donnent comme conséquence immédiate:

$$
\mathcal{Q} P\left(\boldsymbol{D}+\boldsymbol{D}^{*}\right) \subset \mathcal{Q} P(\boldsymbol{C})
$$

Les formules (3) et (4) entraînent: $\mathscr{e}\left(\boldsymbol{D}+\boldsymbol{D}^{*}\right) \subset \mathcal{C}(\boldsymbol{C}) \cdot \mathscr{C}\left(\boldsymbol{S}_{0}\right)$, d'où, suivant le th. $21^{\text {b }}$,

$$
\mathcal{C}\left(\boldsymbol{D} \stackrel{\circ}{+} \boldsymbol{D}^{*}\right) \subset \mathcal{e} \mathcal{P}\left(\boldsymbol{C} \stackrel{\circ}{+} \boldsymbol{S}_{0}\right)
$$

En appliquant une fois encore le th. $28^{\mathrm{b}}$ et en tenant compte du th. $50^{a}$, on obtient:

$$
\mathcal{Q P}\left(\boldsymbol{C}+\boldsymbol{S}_{0}\right)=\mathcal{e} \mathcal{P}\left(\boldsymbol{C}+\boldsymbol{S}_{0}^{*}\right) .
$$

Le th. $23^{\text {b }}$ (pour $\left.\boldsymbol{F} \doteq C+S_{0}^{*}\right)$ implique que $\mathcal{C}\left(C+\boldsymbol{S}_{0}^{*}\right)=$ $=\mathcal{C} \mathcal{P}\left(\boldsymbol{I}+\boldsymbol{C}+\boldsymbol{S}_{0}^{*}\right)=\mathcal{C} \mathcal{P}\left(\boldsymbol{S}_{0}^{*}+[\boldsymbol{I}+\boldsymbol{C}]\right)$. En vertu de l'inclusion évidente: $\boldsymbol{I} \subset^{\circ} \boldsymbol{I}+\boldsymbol{C}$, on conclut du th. $24^{\mathrm{a}}$ (pour $\boldsymbol{F} \rightleftharpoons \boldsymbol{S}_{0}^{*}$ et 
$\boldsymbol{G}=\boldsymbol{I}+\boldsymbol{C})$ que $\left.\mathcal{e}\left(\boldsymbol{S}_{0}^{*}+\stackrel{\boldsymbol{I}}{+} \boldsymbol{C}\right]\right) \subset \mathcal{e}\left(\boldsymbol{S}_{0}^{*}[\boldsymbol{I}+\boldsymbol{C}]\right)$. Par conséquent,

$$
\mathscr{C P}\left(\boldsymbol{C}+\boldsymbol{S}_{0}^{*}\right) \subset \mathscr{e P}\left(\boldsymbol{S}_{0}^{*}[\boldsymbol{I}+\boldsymbol{C}]\right) .
$$

A l'aide du th. $21^{\mathrm{a}}$ on déduit du th. $73^{\mathrm{e}}$ l'inclusion: $\mathfrak{e} \mathcal{C}\left(\boldsymbol{S}_{0}^{*}[\boldsymbol{I} \dot{\boldsymbol{C}}]\right) \subset \mathcal{Q} \mathcal{\ell}(\boldsymbol{D})$, qui, rapprochée de (6) et (7), donne: $\mathscr{C}\left(C+S_{0}\right) \subset \mathscr{e}(D)$. En appliquant à deux reprises le th. $21^{\mathrm{b}}$, on en tire facilement: $\mathscr{C} \mathcal{C}\left(\boldsymbol{C}+\boldsymbol{S}_{0}\right)=\mathcal{Q} \mathcal{P}\left(\boldsymbol{C}+\left[\boldsymbol{C}+\boldsymbol{S}_{0}\right]\right)=\mathcal{Q} \mathscr{C}(\boldsymbol{C})$.

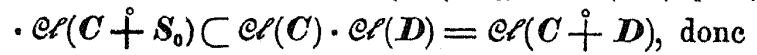

$$
\mathcal{e}\left(C+\boldsymbol{S}_{0}\right) \subset \mathcal{e P}(\boldsymbol{C}+\boldsymbol{D}) \text {. }
$$

Les formules (1), (2); (5) et (8) entraînent tout de suite l'identité cherchée:

$$
\begin{gathered}
\mathscr{e}(\boldsymbol{C}+\boldsymbol{D})=\mathcal{C} \mathcal{P}\left(\boldsymbol{C}+\boldsymbol{D}^{*}\right)=\mathcal{C} \mathcal{C}\left(\boldsymbol{C}+\boldsymbol{D}+\boldsymbol{D}^{*}\right)=\mathcal{e}_{*}\left(\boldsymbol{D}+\boldsymbol{D}^{*}\right)= \\
=\mathfrak{e}\left(\boldsymbol{C}+\boldsymbol{S}_{0}\right) \text {, e. q. f. d. }
\end{gathered}
$$

b) se déduit sans peine de a) par l'application du cor. 22.

Comme eas particuliers du th. $77^{\mathrm{b}}$ citons les identités: $\mathcal{Q}\left(\boldsymbol{C}+\boldsymbol{S}_{\beta}+\boldsymbol{D}\right)=\mathcal{C}\left(\boldsymbol{S}_{\beta}+\boldsymbol{D}+\boldsymbol{D}^{*}\right)=\mathcal{O} \mathcal{P}\left(\boldsymbol{C}+\boldsymbol{S}_{\beta}\right), \mathcal{Q} \mathcal{C}(\boldsymbol{C}+\boldsymbol{S}+\boldsymbol{D})=$ $=\mathfrak{Q} \mathcal{C}(\boldsymbol{C}+\boldsymbol{S})$ etc.

Corollaire 78. a) $\mathcal{C}\left(\boldsymbol{T}+\boldsymbol{D}^{*}\right)=\mathcal{E} \mathcal{\ell}\left(\boldsymbol{T}^{*}+\boldsymbol{D}\right)=\mathcal{Q} \mathcal{P}(\boldsymbol{C}+\boldsymbol{T})$;

b) $\mathfrak{C}\left(\boldsymbol{T}+\boldsymbol{D}^{*}+\boldsymbol{H}\right)=\mathfrak{C}\left(\boldsymbol{T}^{*} \stackrel{\circ}{+} \stackrel{+}{+} \boldsymbol{H}\right)=\mathfrak{e} \mathcal{l}(\boldsymbol{C}+\boldsymbol{T}+\boldsymbol{H})$ pour toute opération $\boldsymbol{H}$.

Démonstration. $\left.{ }^{a}\right)$ En posant dans le th. $77^{\mathrm{b}}: \boldsymbol{H} \rightleftharpoons \boldsymbol{T}$, resp. $\boldsymbol{H}=\boldsymbol{T}^{*}$, on obtient:

(1) $\quad \mathcal{Q}(\boldsymbol{C}+\boldsymbol{D}+\boldsymbol{T})=\mathcal{Q} \mathcal{L}\left(\boldsymbol{D}+\boldsymbol{D}^{*}+\boldsymbol{T}\right)$ et $\mathscr{e} \mathcal{L}\left(\boldsymbol{C}+\boldsymbol{D}^{*}+\boldsymbol{T}^{*}\right)=$ $=\mathcal{E} \mathcal{R}\left(\boldsymbol{D}+\boldsymbol{D}^{*}+\boldsymbol{T}^{*}\right)$.

D'après le th. $73^{\mathrm{d}}$ et le lem. $19^{\circ}$ on a $\boldsymbol{D} \subset \boldsymbol{C}$ et $\boldsymbol{D}^{*} \subset \boldsymbol{T}^{*}$, donc $\boldsymbol{T}+\boldsymbol{D}=\boldsymbol{T}$ et $\boldsymbol{T}^{*}+\boldsymbol{D}^{*} \bumpeq \boldsymbol{T}^{*}$, ce qui permet de simplifier les formules (1) commio suit:

(2) $\quad \mathfrak{e}(\boldsymbol{C}+\boldsymbol{T})=\mathfrak{e}\left(\boldsymbol{T}+\boldsymbol{D}^{*}\right)$ et $\quad \mathcal{e}\left(\boldsymbol{C}+\boldsymbol{T}^{*}\right)=\mathfrak{e} \mathcal{l}\left(\boldsymbol{T}^{*}+\boldsymbol{D}\right)$.

Or, en rapprochant les égalités (2) du th. $41^{a}$, on parvient aussitốt à la formule cherchée. $\left.{ }^{b}\right)$ résulte immédiatemént de ${ }^{a}$ ) en raison du cor. 22.

En tenant compte du th. 41 , on conclat da corollaire précédent que la famille $\mathscr{C}\left(\boldsymbol{T} \stackrel{+}{+} \boldsymbol{D}^{*}\right)=\mathscr{C}\left(\boldsymbol{T}^{*} \stackrel{+}{\boldsymbol{D}}\right)$, de-même que toute famille de la forme $\mathscr{C}\left(\boldsymbol{T}+\boldsymbol{D}^{*}+\boldsymbol{H}\right)=\mathscr{C}\left(\boldsymbol{T}^{*}+\boldsymbol{D}+\boldsymbol{H}\right)$, où $\boldsymbol{H}$ est one opération quelconque envisagée dans cet ouvrage, ne contient que deux éléments, à savuir 0 et $\boldsymbol{U}(1)$.

Théorème 79. a) Si $\beta \geqslant \gamma$, on a $\mathcal{C}\left(\boldsymbol{S}_{\beta}+\boldsymbol{D}\right)=\mathcal{C}\left(\boldsymbol{S}_{\beta}+\boldsymbol{S}_{\gamma}^{*}+\boldsymbol{D}\right)$ et $\mathscr{e}\left(\boldsymbol{S}_{\beta}^{*}+D^{*}\right)=\mathscr{e}\left(\boldsymbol{S}_{\gamma}+\boldsymbol{S}_{\beta}^{*}+D^{*}\right)$;

b) $\mathcal{C}(\boldsymbol{S} \dot{+} \boldsymbol{D})=\mathcal{C} \mathcal{P}\left(\boldsymbol{S}+\boldsymbol{S}^{*}+\boldsymbol{D}\right)=\mathfrak{e}\left(\boldsymbol{S}+\boldsymbol{S}_{\gamma}^{*}+\boldsymbol{D}\right)$ et $\mathcal{O}\left(\boldsymbol{S}^{*}+\boldsymbol{D}^{*}\right)=$ $=\mathscr{e}\left(S+S^{*}+D^{*}\right)=\mathcal{C}_{\iota}\left(S_{\gamma}+S^{*}+D^{*}\right)$.

Démonstration. ${ }^{a}$ ) En raison du th. $52^{\mathrm{b}}$ et du lem. 19e, on a $\boldsymbol{S}_{\gamma}^{*} \subset \boldsymbol{S}_{\beta}^{*}$, d'où en vertu du th. $73^{\mathrm{g}} \boldsymbol{S}_{\gamma}^{*} \mathcal{C}^{\circ} \boldsymbol{D} \boldsymbol{S}_{\beta} \boldsymbol{D}$; à l'aide du th. $21^{\mathrm{a}}$ on en conclut que

$$
\mathscr{C} \mathcal{L}\left(\boldsymbol{D} \boldsymbol{S}_{\beta} \boldsymbol{D}\right) \stackrel{\circ}{\complement} \mathcal{C}\left(\boldsymbol{S}_{\gamma}^{*}\right)
$$

En posant dans le th. $24^{\mathrm{a}}$ : $\boldsymbol{F} \doteq \boldsymbol{D}$ et $\boldsymbol{G}=\boldsymbol{S}_{\beta} \boldsymbol{D}$ et en tenant compte du th. $73^{\mathrm{a}}$, on tire: $\mathcal{e}\left(\boldsymbol{D}+\boldsymbol{S}_{\beta} \boldsymbol{D}\right) \subset \mathcal{Q} \mathcal{\ell}\left(\boldsymbol{D} \boldsymbol{S}_{\beta} \boldsymbol{D}\right)$; comme, de plus, $\mathscr{C}\left(D+S_{\beta} D\right)=\mathscr{C}(D) \cdot \mathscr{C}\left(S_{\beta} D\right)$ d'après le th. $21^{\text {b. }}$, on a:

$$
\mathfrak{Q}(D) \cdot \mathcal{C}\left(\boldsymbol{S}_{\beta} D\right) \subset \mathcal{Q} \ell\left(D \mathbf{S}_{\beta} D\right)
$$

Le th. $24^{\text {a }}$ donne encore en vertu da th. $73^{\text {b }}: \mathcal{E}\left(S_{\beta}+D\right) C$ $\mathcal{C} \mathcal{P}\left(\boldsymbol{S}_{8} \boldsymbol{D}\right)$; il résulte en outre da th. $21^{\mathrm{a}}$ (ou $\left.21^{\mathrm{b}}\right)$ que $\mathcal{Q}\left(\boldsymbol{S}_{\beta}+\boldsymbol{D}\right) \mathcal{C}$ $\mathcal{C} \mathscr{C}(\boldsymbol{D})$. Par conséquent,

$$
\mathcal{C}\left(\boldsymbol{S}_{\boldsymbol{\beta}}+\boldsymbol{D}\right) \subset \mathcal{e}(\boldsymbol{D}) \cdot \mathcal{C}\left(\boldsymbol{S}_{\beta} \boldsymbol{D}\right)
$$

Les inclusions (1)-(3) entraînent aussitôt:

$$
\mathcal{O} \mathcal{P}\left(\boldsymbol{S}_{\beta}+\boldsymbol{D}\right) \subset \mathcal{Q} \mathcal{P}\left(\boldsymbol{S}_{\gamma}^{*}\right) \text {. }
$$

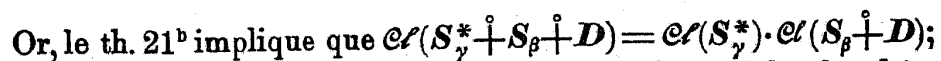
en le rapprochant de (4), on obtient la première formule cherchée:

$$
\mathcal{Q}\left(\boldsymbol{S}_{\boldsymbol{B}}+\boldsymbol{D}\right)=\mathcal{O} \mathcal{C}\left(\boldsymbol{S}_{\beta}+\boldsymbol{S}_{\gamma}^{*}+\boldsymbol{D}\right) \text {. }
$$

D'après le cor. 27 , il s'en suit que $\mathscr{C}\left(\left[S_{\beta}+D\right]^{*}\right)=$ 
$=\mathscr{e}\left(\left[\boldsymbol{S}_{\beta}+\boldsymbol{S}_{\gamma}^{*}+\boldsymbol{D}\right]^{*}\right)$, d'où l'ón déduit à l'aide du lem. $19^{\mathrm{b}, \mathrm{d}}$ la seconde formule:

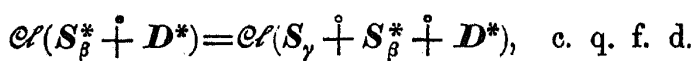

b) Soit $\boldsymbol{\kappa}_{\alpha}$ la puissance de l'ensemble universel 1. Posons: $\beta=$ $=\max (\alpha+1, \gamma)$, d'où $\beta \geqslant \gamma$. Lies formules ${ }^{\text {a) }}$ qui viennent d'etre établies donnent alors

(5) $\quad \mathscr{C}\left(\boldsymbol{S}_{\beta}+\boldsymbol{D}\right)=\mathscr{C}\left(\boldsymbol{S}_{\beta}+\boldsymbol{S}_{\beta}^{*}+\boldsymbol{D}\right)=\mathscr{e}\left(\boldsymbol{S}_{\beta}+\boldsymbol{S}_{\gamma}^{*}+\boldsymbol{D}\right)$ et $\mathfrak{C}\left(\boldsymbol{S}_{\beta}^{*}+D^{*}\right)=\mathcal{Q} \mathcal{C}\left(\boldsymbol{S}_{\beta}+\boldsymbol{S}_{\beta}^{*}+\boldsymbol{D}^{*}\right)=\mathcal{Q} \mathcal{L}\left(\boldsymbol{S}_{\gamma}+\boldsymbol{S}_{\beta}^{*}+\boldsymbol{D}^{*}\right)$.

Comme, en vertu de la définition de $\beta$, on a $\overline{\bar{I}}<\aleph_{\beta}$, le th. $51^{\text {d }}$ entraîne: $\boldsymbol{S}_{\beta} \rightleftharpoons \boldsymbol{S}$ et, par conséquent, $\boldsymbol{S}_{\beta}^{*}=\boldsymbol{S}^{*}$. En remplaçant donc dans (5) $\boldsymbol{S}_{\beta}$ par $\boldsymbol{S}$ et $\boldsymbol{S}_{\beta}^{*}$ par $\boldsymbol{S}^{*}$, on parvieut aussitôt aux identités cherchées.

Le théorème suivant nous permettra de résumer dans la suite (a la fin de ce §) d'une manière bien claire tous les résultats acquis dans cet ouvrage.

Théorème 80. Soit $\Re$ la classe composée d'opérations $\boldsymbol{C}, \boldsymbol{T}, \boldsymbol{T}^{*}$, $\boldsymbol{S}, \boldsymbol{S}^{*}$ et de toutes les opérations $\boldsymbol{S}_{\xi}$ et $\boldsymbol{S}_{\eta}^{*}$ ( $\xi$ et $\eta$ étant des nombres ordinaux arbitraires); soit $\mathbb{L}$ la classe formée d'opérations $\boldsymbol{C}, \boldsymbol{T}$, $\boldsymbol{T}^{*}, \boldsymbol{C}+\boldsymbol{T}$ et de toutes les opérations $\boldsymbol{S}_{\xi}, \boldsymbol{S}_{\eta}^{*}, \boldsymbol{C}+\boldsymbol{S}_{\xi}, \boldsymbol{T}+\boldsymbol{S}_{\xi}$, $\boldsymbol{T}^{*}+\boldsymbol{S}_{\eta}^{*}, \boldsymbol{S}_{\xi}+\boldsymbol{S}_{\eta}^{*}, \boldsymbol{S}_{\xi}+D^{*}, S_{\eta}^{*}+D, S_{\xi}+S_{\eta}^{*}+D$ et $\boldsymbol{S}_{\xi}^{\circ}+\boldsymbol{S}_{\eta}^{*}+D^{*}$ ( $\xi$ et $\eta$ arbitraires). On a alors:

$$
\underset{\mathcal{O}\left(\boldsymbol{F}^{\prime}\right)}{E}\left[\boldsymbol{F}^{\prime} \in \mathfrak{S}(\mathfrak{N})\right]=\underset{\mathcal{Q} \mathcal{\ell}(\boldsymbol{G})}{E}[\boldsymbol{G} \in \mathfrak{L}] .
$$

Démonstration. Considérons une famille arbitraire $\mathfrak{Q}$ telle que

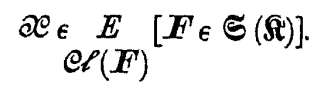

Conformément à la déf. $11^{\mathrm{a}}$, (1) implique l'existence d'une classe d'opérations $\mathbb{G}_{1}$ vérifiant les formules:

$$
\mathfrak{e}=\mathfrak{e}\left(\sum_{G \in \mathbb{S}_{1}}^{\circ} G\right)
$$

$$
\mathbb{S}_{1} \subset \Omega \text { et } \quad \mathscr{G}_{1} \neq 0 .
$$

Or, nous allons prouver qu'ils existent une classe d'opérations $\mathbb{C}_{2}$ et deux nombres ordinaux $\beta$ et $\gamma$ qui remplissent les conditions suivantes:

$$
\sum_{G_{\in} \mathbb{S}_{2}}^{\circ} G=\sum_{G \in \mathbb{S}_{1}}^{\circ} G
$$

$$
\mathbb{B S}_{2} \subset\left\{C, \boldsymbol{T}, \boldsymbol{T}^{*}, \boldsymbol{S}_{\beta}, \boldsymbol{S}_{\gamma}^{*}, \boldsymbol{D}, \boldsymbol{D}^{*}\right\} \text { et } \mathfrak{G}_{\mathbf{2}} \neq 0 .
$$

Il résulte, en effet, de (3) et de l'hypothèse du théorème que $\mathfrak{S}_{1}$ ne contient que les opérations $C, T, T^{*}, S, S^{*}, D, D^{*}$ ainsi que les opérations de la forme $\boldsymbol{S}_{\xi}$ et $\boldsymbol{S}_{\eta}^{*}$. Les opérations $\boldsymbol{S}$ et $\boldsymbol{S}^{*}$ peuvent être représentées sous lá forme de $\boldsymbol{S}_{\xi}$, resp. $\boldsymbol{S}_{\eta}^{*}$ (puisque, $\boldsymbol{N}_{\alpha}$ étaut la puissance de l'ensemble universel 1 , on a d'après le th. $51^{\text {da }}$ $\boldsymbol{S} \rightleftharpoons \boldsymbol{S}_{a+1}$ et $\boldsymbol{S}^{*} \rightleftharpoons \boldsymbol{S}_{a+1}^{*}$ ); e'est pourqui on pent les négliger ici. Soient maintenant $\mathfrak{F}_{1}$, resp. $\mathfrak{F}_{2}$, la classe de toutes les opérations de $\mathscr{G}_{1}$ qui sont de la forme $S_{\xi}$, resp. $S_{\eta}^{*}$, et $A_{1}$, resp. $A_{q}$, l'ensemble de tous les indices $\xi$, resp. $\eta$, de ces opérations. Posons: $\beta=\sup \left(A_{1}\right)$ et $\gamma=\sup \left(A_{2}\right) ; \quad \mathfrak{G}_{2}=\mathfrak{G}_{1}$, lorsque $\mathfrak{\mho}_{1}=0=\mathfrak{W}_{2} ;$ $\mathfrak{G}_{2}=\left(\mathfrak{G}_{1}-\widetilde{W}_{1}\right)+\left\{\boldsymbol{S}_{\beta}\right\}$, lorsque $\mathfrak{F}_{1} \neq 0$ et $\tilde{F}_{2}=0 ; \mathfrak{G}_{2}=\left(\mathscr{G}_{1}-\mathfrak{F}_{2}\right)+$ $+\left\{\boldsymbol{S}_{\gamma}^{*}\right\}$, lorsque $\mathfrak{F}_{1}=0$ et $\mathfrak{F}_{2} \neq 0$, et enfin $\mathfrak{G}_{2}=\left(\mathfrak{G}_{1}-\left(\mathfrak{F}_{1}+\mathfrak{F}_{2}\right)\right)+$ $+\left\{\boldsymbol{S}_{\beta}, \boldsymbol{S}_{\gamma}^{* \prime}\right.$, lorsque $\mathfrak{\mho}_{1} \neq 0$ et $\mathfrak{\mho}_{2} \neq 0$. Il résulte du th. $52^{\mathrm{a}}$ que $S_{\beta}=\sum_{G \in \mathfrak{F}_{1}}^{\circ} G$ pour $\mathfrak{F}_{1} \neq 0$ et de-même (en vertu du lem. 19d) que $S_{\gamma}^{*}=\sum_{G \in \mathfrak{F}_{q}}^{0} G$ pour $\mathfrak{\mho}_{z} \neq 0$. On on déduit sans peine l'égalité (4) et on conclut tout de saite de la définition de $\mathbb{S S}_{2}$ que cette classe vérifie aussi les formules (5).

Notons à présent les identités suivantes qui existent entre les diverses familles de la forme $\mathcal{C}\left(\sum_{G \in \in \mathbb{S}}^{\circ} G\right)$, où $\mathscr{S}$ est une sous-classe de $\left\{C, T, T^{*}, S_{\beta}, S_{\gamma}^{*}, D, D^{*}\right\}$ :

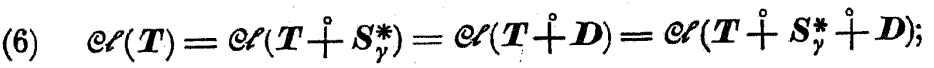

(7) $\mathscr{C}\left(\boldsymbol{T}+\boldsymbol{S}_{\beta}\right)=\mathscr{e}\left(\boldsymbol{T}+\boldsymbol{S}_{\beta}+\boldsymbol{S}_{\gamma}^{*}\right)=\mathscr{e}\left(\boldsymbol{T}+\boldsymbol{S}_{\beta}+\boldsymbol{D}\right)=$ $=\mathcal{e}\left(\boldsymbol{T}+\boldsymbol{S}_{\beta}+\boldsymbol{S}_{\gamma}^{*}+\boldsymbol{D}\right)$;

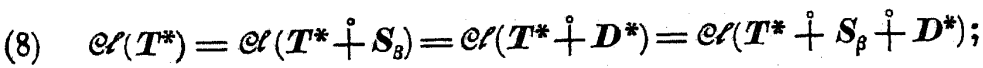


(9) $\quad \mathscr{C}\left(\boldsymbol{T}^{*}+\boldsymbol{S}_{\gamma}^{*}\right)=\mathfrak{e}\left(\boldsymbol{T}^{*}+\boldsymbol{S}_{\beta}+\boldsymbol{S}_{\gamma}^{*}\right)=\mathfrak{Q} \mathcal{\ell}\left(\boldsymbol{T}^{*}+\boldsymbol{S}_{\gamma}^{*}+\boldsymbol{D}^{*}\right)=$ $=\mathcal{e}\left(\boldsymbol{T}^{*}+\boldsymbol{S}_{\beta}+\boldsymbol{S}_{\gamma}^{*}+\boldsymbol{D}^{*}\right)$;

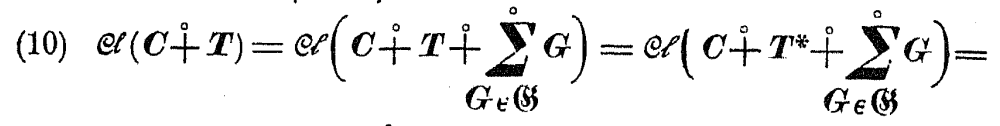

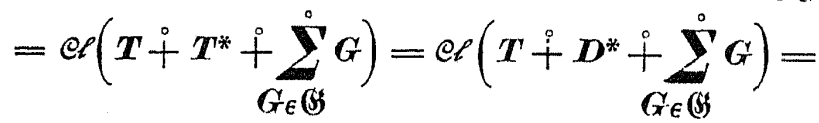
$=\mathcal{O P}\left(\boldsymbol{T}^{*}+\boldsymbol{D}+\sum_{\boldsymbol{G}_{\in \in \mathbb{S}}}^{\circ} \boldsymbol{G}\right)$ pour toute sous-classe (S) (vide ou non) de $\left\{\boldsymbol{C}, \boldsymbol{T}, \boldsymbol{T}^{*}, \boldsymbol{S}_{\beta}, \boldsymbol{S}_{\gamma}^{*}, \boldsymbol{D}, \boldsymbol{D}^{*}\right\}$;

(11) $\mathscr{e}\left(\boldsymbol{C}+\boldsymbol{S}_{0}\right)=\mathfrak{C} \mathcal{C}(\boldsymbol{C}+\boldsymbol{D})=\mathfrak{e} \mathcal{l}\left(\boldsymbol{C}+\boldsymbol{D}^{*}\right)=\mathfrak{e} \mathcal{l}\left(\boldsymbol{D}+\boldsymbol{D}^{*}\right)=$ $=\mathcal{O}\left(\boldsymbol{C}+\boldsymbol{D}+\boldsymbol{D}^{*}\right)$

(12) $\mathscr{C}\left(\boldsymbol{C}+\boldsymbol{S}_{\beta}\right)=\mathscr{Q} \mathcal{Q}\left(\boldsymbol{C}+\boldsymbol{S}_{\beta}+\boldsymbol{D}\right)=\mathscr{Q} \mathcal{P}\left(\boldsymbol{C}+\boldsymbol{S}_{\beta}+\boldsymbol{D}^{*}\right)=$

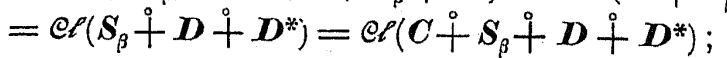

(13) $\mathcal{Q} \mathcal{C}\left(\boldsymbol{C}+\boldsymbol{S}_{\gamma}\right)=\mathcal{Q} \mathcal{C}\left(\boldsymbol{C}+\boldsymbol{S}_{\gamma}^{*}\right)=\mathcal{Q} \mathcal{L}\left(\boldsymbol{C}+\boldsymbol{S}_{\gamma}^{*}+\boldsymbol{D}\right)=\mathfrak{Q} \mathcal{L}\left(\boldsymbol{C}+\boldsymbol{S}_{\gamma}^{*}+\boldsymbol{D}^{*}\right)=$ $=\mathcal{Q} \mathcal{P}\left(\boldsymbol{S}_{\gamma}^{*}+\boldsymbol{D}+\boldsymbol{D}^{*}\right)=\mathcal{Q} \mathcal{\ell}\left(\boldsymbol{C}+\boldsymbol{S}_{\gamma}^{*}+\boldsymbol{D}+\boldsymbol{D}^{*}\right)$;

(14) $\quad \mathfrak{e}\left(\boldsymbol{C}+\boldsymbol{S}_{\max (\beta, \gamma)}\right)=\mathfrak{e}\left(\boldsymbol{C}+\boldsymbol{S}_{\beta}+\boldsymbol{S}_{\gamma}^{*}\right)=\mathscr{e}\left(\boldsymbol{C}+\boldsymbol{S}_{\beta}+\boldsymbol{S}_{\gamma}^{*}+\boldsymbol{D}\right)=$ $=\mathscr{C}\left(\boldsymbol{C}+\boldsymbol{S}_{\beta}+\boldsymbol{S}_{\gamma}^{*}+\boldsymbol{D}^{*}\right)=\mathscr{Q} \mathcal{C}\left(\boldsymbol{S}_{\beta}+\boldsymbol{S}_{\gamma}^{*}+\boldsymbol{D}+\boldsymbol{D}^{*}\right)=$ $=\mathcal{e}\left(\boldsymbol{C}+\boldsymbol{S}_{\beta}+\boldsymbol{S}_{\gamma}^{*}+\boldsymbol{D}+\boldsymbol{D}^{*}\right)$;

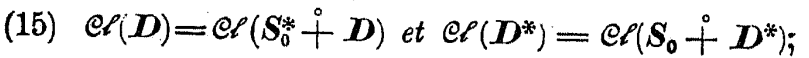

(16) $\mathcal{C}\left(\boldsymbol{S}_{\beta}+\boldsymbol{D}\right)=\mathscr{e}\left(\boldsymbol{S}_{\beta}+\boldsymbol{S}_{\beta}^{*}+\boldsymbol{D}\right)$ et $\quad \mathcal{Q}\left(\boldsymbol{S}_{\gamma}^{*}+\boldsymbol{D}^{*}\right)=$ $=\mathscr{e}\left(\boldsymbol{S}_{\gamma}+\boldsymbol{S}_{\gamma}^{*}+\boldsymbol{D}^{*}\right)$.

On démontre ces formules comme il suit:

Les identités (6)-(9) résultent des inclusions: $\boldsymbol{S}_{\gamma}^{*} \mathcal{\complement}^{\circ} \boldsymbol{T}, \boldsymbol{D} \subset^{\circ} \boldsymbol{T}$, $\boldsymbol{S}_{\beta} \check{\complement}^{\circ} \boldsymbol{T}^{*}$ et $\boldsymbol{D}^{*} \mathcal{C}^{\circ} \boldsymbol{T}^{*}$ (th. $51^{\mathrm{b}}$, th. $73^{\mathrm{d}}$, lem. $19^{\mathrm{b}, \mathrm{c}}$ ), qui en vertu du lem. 13 entraînent les égalités: $\boldsymbol{T} \varrho \boldsymbol{T}+\boldsymbol{S}_{\gamma}^{*}=\boldsymbol{T}+\boldsymbol{D}$ et de-même $T^{*}=T^{*}+S_{\beta}=T^{*}+D^{*}$.

Pour démontrer la formule (10), il est à noter que pour toute opération $\boldsymbol{G}$ de la classe $\left\{\boldsymbol{C}, \boldsymbol{T}, \boldsymbol{T}^{*}, \boldsymbol{S}_{\beta}, \boldsymbol{S}_{\gamma}^{*}, \boldsymbol{D}, \boldsymbol{D}^{*}\right\}$ on a $\boldsymbol{G}(0)=0$ (lem. $18^{\mathrm{d}}$ et $19^{\mathrm{k}}$, th. $36^{\mathrm{c}}, 50^{\mathrm{c}}$ et $73^{\mathrm{c}}$ ); il en résulte d'après la déf. $9^{\mathrm{b}}$ que l'on a aussi $\left[\sum_{G \in \mathbb{S}}^{\circ} G\right](0)=0$ pour toute sous-classe (S) de cette classe. En posant done dans le th. $41^{\mathrm{b}}$ et le cor. $78^{\mathrm{b}}: H=\sum_{G \in \mathbb{G}}^{\circ} G$

et en appliquant le th. $41^{\mathrm{a}}$, on obtient la furmule cherchée.

Les identités (11) sont établies dans le th. 77a . En posant dans le th. $77^{\mathrm{b}}: \boldsymbol{H}=\boldsymbol{S}_{\beta}$ et en tenant compte de l'inclusion: $\boldsymbol{S}_{0} \stackrel{\bullet}{\complement} \boldsymbol{S}_{\beta}$ (th. $52^{b}$ ), on parvient à la formule (12). Les formules (13) et (14) s'obtiennent d'une façon tout à fait analogue $\left(\boldsymbol{H}=\boldsymbol{S}_{\gamma}^{*}\right.$, resp. $\boldsymbol{H}=$ $=\boldsymbol{S}_{\beta}+\boldsymbol{S}_{\gamma}^{*}$ ), mais on aura soin de remarquer qu'en raison des th. $28^{\mathrm{b}}$ et $50^{\mathrm{a}} \mathfrak{e} \mathcal{C}\left(\boldsymbol{C}+\boldsymbol{S}_{\gamma}\right)=\mathcal{C} \mathcal{C}\left(\boldsymbol{C} \stackrel{+}{+} \boldsymbol{S}_{\gamma}^{*}\right)$, d'où, en vertu du th. $52^{\mathrm{a}}$ et du cor. 22 (pour $\boldsymbol{F} \rightleftharpoons \boldsymbol{C}+\boldsymbol{S}_{\gamma}, \boldsymbol{G}=\boldsymbol{C}+\boldsymbol{S}_{\gamma}^{*}$ et $\boldsymbol{H} \cong \boldsymbol{S}_{\beta}$ ), $\mathcal{Q} \mathcal{C}\left(\boldsymbol{C}+\boldsymbol{S}_{\max (\beta, \gamma, \gamma)}\right)=\mathcal{Q} \mathcal{P}\left(\boldsymbol{C}+\boldsymbol{S}_{\gamma}+\boldsymbol{S}_{\beta}\right)=\mathcal{Q} \mathcal{C}\left(\boldsymbol{C}+\boldsymbol{S}_{\gamma}^{*}+\boldsymbol{S}_{\beta}\right)$.

Les égalites (15) se déduisent sans peine des th. 76 et $21^{\mathrm{b}}$ (pour $\boldsymbol{F}=\boldsymbol{S}_{0}^{*}$ et $\boldsymbol{G}=\boldsymbol{D}$, resp. pour $\boldsymbol{F} \rightleftharpoons \boldsymbol{S}_{0}$ et $\boldsymbol{G = 0} \boldsymbol{D}^{*}$ ). Enfin, les formules (16) ne présentent que des cas particuliers du th. $79^{\mathrm{a}}$.

Or, on s'aperçoit sans peine qu'en conséquence de la déf. $9^{a, b}$ et des identités (6)-(16)。 qui viennent d'être établies, toute famille de la forme $\mathcal{e}\left(\sum_{G \in \mathbb{S}} G\right)$, où $\mathfrak{G}$ est une sous-classe non-vide de $\left\{\boldsymbol{C}, \boldsymbol{T}, \boldsymbol{T}^{*}, \boldsymbol{S}_{\beta}, \boldsymbol{S}_{\gamma}^{*}, \boldsymbol{D}, \boldsymbol{D}^{*}\right\}$, coïncide avec une des familles suivantes: $\quad \mathcal{P}(\boldsymbol{C}), \quad \mathcal{C}(\boldsymbol{T}), \quad \mathcal{Q} \mathcal{P}\left(\boldsymbol{T}^{*}\right), \quad \mathcal{Q} \mathcal{P}\left(\boldsymbol{S}_{\beta}\right), \quad \mathcal{e} \mathcal{l}\left(\boldsymbol{S}_{\gamma}^{*}\right)$,

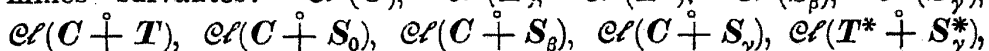

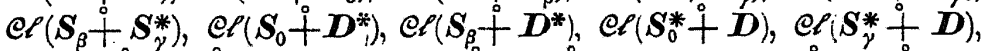
$\mathcal{C} \mathcal{l}\left(\boldsymbol{S}_{\beta}+\boldsymbol{S}_{\beta}^{*}+\boldsymbol{D}\right), \quad \mathcal{O} \mathcal{P}\left(\boldsymbol{S}_{\beta}+\boldsymbol{S}_{\gamma}^{*}+\boldsymbol{D}\right), \quad \mathcal{Q}\left(\boldsymbol{S}_{\beta}+\boldsymbol{S}_{\gamma}^{*}+\boldsymbol{D}^{*}\right)$ et $\mathscr{C}\left(\boldsymbol{S}_{\gamma}+\boldsymbol{S}_{\gamma}^{*}+\boldsymbol{D}^{*}\right)$, done, conformément à l'hypothèse, avec une des familles $\mathcal{Q} \mathcal{C}(\boldsymbol{G})$ où $\boldsymbol{G} \in \mathbb{L}$. Cela s'applique, en particulier, à la famille $\mathcal{C}\left(\sum_{G \in \mathfrak{G}_{2}}^{\circ} G\right), \mathfrak{G}_{2}$ étant selon (5) une sous-classe non-vide de $\left\{\boldsymbol{C}, \boldsymbol{T}, \boldsymbol{T}^{*}, \boldsymbol{S}_{\beta}, \boldsymbol{S}_{\gamma}^{*}, \boldsymbol{D}, \boldsymbol{D}^{*}\right\} ;$ il existe donc une opération $\boldsymbol{G}^{\prime}$ qui remplit les formules:

et

$$
\mathscr{e}\left(\sum_{G \in \mathbb{G}_{2}}^{\circ} G\right)=\mathscr{e}\left(G^{\prime}\right)
$$$$
G^{\prime} \in \mathbb{L} \text {. }
$$

Les égalités (2), (4) et (17) donnent tout de suite: $\mathfrak{e}=\mathfrak{C}\left(\boldsymbol{G}^{\prime}\right)$ : en le rapprochant de (18), on obtient:

$$
\mathfrak{Q P} \in \underset{\mathcal{C} P(G)}{E}[\boldsymbol{G} \in \mathbb{L}]
$$


Il est ainsi prouvé que la formule (1) implique toujours (19); par conséquent, on a l'inclusion: $\underset{\mathcal{Q}(\boldsymbol{F})}{E}[\boldsymbol{F} \in \mathbb{S}(\mathbb{R})] \underset{\mathcal{Q} \mathcal{P}(\boldsymbol{G})}{\boldsymbol{E}}[\boldsymbol{G} \in \mathbb{L}]$. L'inclusion inverse résultant de l'hypothèse d'une façon immédiate, on parvient finalement à lidentité cherchée:

$$
\underset{\mathscr{C}\left(\boldsymbol{F}^{\prime}\right)}{E[\boldsymbol{F} \in \mathcal{S}(\Re)]}=\underset{\mathscr{C} \mathcal{L}(\boldsymbol{G})}{E}[\boldsymbol{G} \in \mathfrak{L}], \text { c. q. f. d. }
$$

Les problèmes concernant la puissance des familles de la forme $\cdot \mathcal{Q}(\boldsymbol{F})$ ne comporteront pas dans ce $\S$ de difficultés appréciables et n'exigeront pas de nouvelles méthodes du raisonnement. On remarquera que les familles de la forme $\mathcal{E}\left(\boldsymbol{F}^{\prime}\right)$, où $\boldsymbol{F}$ est une somme d'opérations qui contient parmi ses sommandes soit les deux opérations $\boldsymbol{D}$ et $\boldsymbol{D}^{*}$, soit uné d'elles accompagnées de $\boldsymbol{C}, \boldsymbol{T}$ ou de $\boldsymbol{T}^{*}$, peuvent être omises dans l'étude de ces problèmes, car en vertu du th. 77 et du cor. 78 ces familles coìncident avec celles qui ont été déjà exarninées dans les $\S \S 4-6$. Ainsi p. ex., en confrontant les th. IX et $77^{\mathrm{a}}$, on se convaint immédiatement que dans l'hypothèse: $\overline{\bar{I}}=\aleph_{\alpha}$ la famille $\mathscr{e} \mathcal{P}(\boldsymbol{C}+\boldsymbol{D})=\mathcal{Q} \mathcal{L}\left(\boldsymbol{C}+\boldsymbol{D}^{*}\right)=\mathcal{C} \mathcal{P}\left(\boldsymbol{D}+\boldsymbol{D}^{*}\right)$ est de puissance $2^{2{ }^{*} \alpha}$

Théorème fondamental XIII. Si $\overline{\bar{I}}=\kappa_{\alpha}$, on $a$

a) $\overline{\overline{\mathcal{Q}(\bar{D})}}=\overline{\overline{\mathcal{Q} \ell\left(\boldsymbol{D}^{*}\right)}}=2^{2^{\mathrm{Na}}}$;

b) $\overline{\overline{\mathscr{Q}\left(\boldsymbol{S}+\boldsymbol{D}^{*}\right)}}=\overline{\overline{\mathscr{Q} \mathcal{Q}\left(\boldsymbol{S}^{*}+\boldsymbol{D}\right)}}=2^{2^{* \alpha}}$;

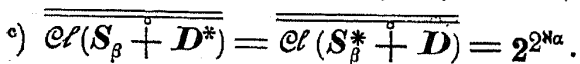

Démonstration. D'après le th. $73^{\mathrm{d}}$ et le lem. $19^{\mathrm{c}}$, on a

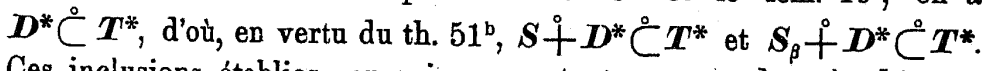
Ces inclusions établies, on raisonnera tout comme dans la démonstration $d u$ th. fond. IV.

Théorème fondamental XIV. Si $\overline{\overline{1}}=\aleph_{\alpha}$, on a $\overline{\overline{\mathcal{C P}(S+D)}}=$ $\left.=\overline{\overline{\mathcal{O}\left(S^{*}+D^{*}\right.}}\right)=2^{* \alpha}$.

Démonstration. En raison du th. $73^{\mathrm{d}}$, on a $\boldsymbol{S}+\boldsymbol{D} \subset^{\circ} \boldsymbol{X}+\boldsymbol{+} S$; d'où, en vertu du th. $21^{\text {a }}, \mathscr{e l}(\boldsymbol{T}-\boldsymbol{i} \boldsymbol{S}) \mathcal{C} \mathscr{C}(\boldsymbol{S}+\boldsymbol{D})$; suivant les th. $79^{b}$ et $21^{\text {b }}$ on a de plus: $\mathscr{C}(S \stackrel{D}{+})=\mathscr{C} \mathcal{P}\left(S \stackrel{+}{+} S^{*}+D\right)=$
$=\mathscr{Q} \mathcal{\ell}\left(\boldsymbol{S}+\boldsymbol{S}^{*}\right) \cdot \mathcal{E} \mathcal{R}(\boldsymbol{D}) \subset \mathcal{Q} \mathcal{P}\left(\boldsymbol{S} \stackrel{\circ}{+} \boldsymbol{S}^{*}\right)$. Il s'en suit aussitôt que

$$
\overline{\overline{\mathscr{C}(\boldsymbol{T}+\boldsymbol{S})}} \leqslant \overline{\overline{\mathcal{Q} \ell(\boldsymbol{S}+\boldsymbol{D})}} \leqslant \overline{\overline{\mathcal{Q} \ell\left(\boldsymbol{S}+\boldsymbol{S}^{*}\right)}}
$$

Conformément aux th. fond. $\mathrm{V}$ et VI, $\overline{\overline{\mathcal{Q P}(\boldsymbol{T}+\boldsymbol{S})}}=2^{\mathrm{Na}}=$ $=\overline{\overline{\mathcal{Q}\left(\boldsymbol{S}+\boldsymbol{S}^{*}\right)}}$, ce qui donne selon (1): $\overline{\overline{\mathcal{Q P}\left(\boldsymbol{S}+\boldsymbol{D}_{)}\right.}}=2^{\mathrm{N \alpha}}$. Comme en outre, d'apres le th. 33 et le lem. 19d, $\overline{\overline{\mathcal{Q} \ell\left(S^{*}+D^{*}\right)}}=\overline{\mathcal{Q} \mathcal{C}(\boldsymbol{S}+D)}$, on déduit finalement:

$$
\overline{\overline{\mathcal{C}}(S+D)}=\overline{\overline{\mathcal{Q}}\left(\boldsymbol{S}^{*}+\boldsymbol{D}^{*}\right)}=2^{\mathrm{\alpha} \alpha}, \text { c. q. f. d. }
$$

Dans le cas où $\overline{\bar{I}}=\mathfrak{a}<\aleph_{0}$ on peut établir pour les puissances des familles étudiées dans le th. XIII les mêmes délimitations qui ont été indiquées plas haut (p. 232) pour la famille $\mathcal{Q}(\boldsymbol{T})$; quant aux familles du th. XIV, les délimitations établies pour la famille $\mathcal{Q} \mathcal{C}\left(\boldsymbol{S}+\boldsymbol{S}^{*}\right)($ p 245) restent valables pour elles.

Théorème fondamental XV. Si $\overline{\overline{1}}=\aleph_{a}$ et $\beta \leqslant p(a)$, on $a$

a) $\overline{\overline{\mathcal{Q}\left(\boldsymbol{S}_{\beta}+\overline{\boldsymbol{D}}\right)}}=\overline{\overline{\mathcal{Q} \mathcal{Q}\left(\boldsymbol{S}_{\beta}^{*}+\overline{\left.D^{*}\right)}\right.}}=2^{2^{\mathrm{N}^{\alpha} \alpha}}$;

v) $\left.\overline{\overline{\mathscr{C}\left(S+\boldsymbol{S}^{\circ}+\boldsymbol{S}_{\beta}^{*}+D^{*}\right.}}\right)=\overline{\overline{\mathcal{Q}\left(\boldsymbol{S}^{*}+\boldsymbol{S}_{\beta}+\boldsymbol{D}\right)}}=2^{2^{\mathrm{N \alpha} \alpha}}$;

c) $\overline{\mathcal{C} \ell\left(\boldsymbol{S}_{\beta}+\overline{\boldsymbol{S}_{\gamma}^{*}+\boldsymbol{D}}\right)}=\overline{\overline{\mathcal{Q} \ell\left(\boldsymbol{S}_{\gamma}+\boldsymbol{S}_{\beta}^{*}+D^{*}\right)}}=2^{2^{\mathrm{Na} a}}$.

Démonstration. A l'aide des th. $51^{\mathrm{b}}, 73^{\mathrm{d}}$ et du lem. $19^{\mathrm{b}, \mathrm{c}}$ on se convaint sans peine, que, $F$ étant une des six opérations suivantes: $S_{\beta}+D, S_{\beta}^{*} \stackrel{+}{+} D^{*}, S+S_{\beta}^{*}+D^{*}, S^{*}+S_{\beta}+D, S_{\beta}+$ $\stackrel{+}{+} \boldsymbol{S}_{\gamma}^{*}+\boldsymbol{D}$ et $\boldsymbol{S}_{\gamma}+\boldsymbol{S}_{\beta}^{*} \stackrel{\circ}{+} \boldsymbol{D}^{*}$, on a soit $\boldsymbol{F}^{\circ} \check{C}^{\boldsymbol{T}} \stackrel{+}{+} \boldsymbol{S}_{\boldsymbol{\beta}}$, soit

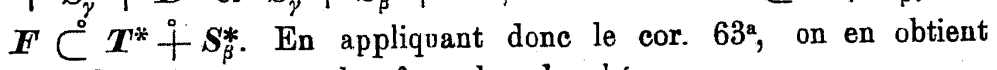
immédiatement toutes les formules cherchées.

La question, si l'on peut renforcer le th. XVa dans le même sens que le th. $X^{b}$ (c. a-d. si l'on pent remplacer dans le th. $67 S_{y}^{*}$ par $D$ ), reste ouverte; ce problème est d'ailleurs équivalent au problème analogue concernant l'opération $C$ (cf. p. 275 et 276 ).

Théorème 81. Si $\overline{\overline{1}}=\aleph_{\alpha}$ et $\beta \leqslant p(\alpha)$, on a $\overline{\overline{\mathcal{Q}\left(\boldsymbol{R}_{\beta}+\overline{+D}\right)}}=$ $=\overline{\overline{\mathcal{Q}}\left(\boldsymbol{\boldsymbol { R }}_{\beta}+\boldsymbol{D}^{*}\right)}=2^{2^{2 \alpha}}$. . 
Démonstration est basée sur les formules: $\boldsymbol{R}_{\beta}+\boldsymbol{D} \subset^{\circ} \boldsymbol{T} \boldsymbol{S}_{\beta}$. $\boldsymbol{R}_{\beta} \stackrel{\circ}{+} \boldsymbol{D}^{*} \check{\complement}^{\circ} \boldsymbol{T}^{*} \boldsymbol{S}_{\beta}^{*}$ et ne diffère pas de celle du th. 64 (resp. du thẻorème précédent).

Thérème 82. Si $\overline{\overline{1}}=\aleph_{\alpha}$ et $\beta>c f(\alpha)$, on a

a) $2^{\text {*a }} \leqslant \overline{\overline{\mathscr{Q P}\left(\boldsymbol{S}_{\beta}+\boldsymbol{D}\right)}}=\overline{\overline{\mathcal{Q P}\left(\boldsymbol{S}_{\beta}^{*}+D^{*}\right)}} \leqslant 2^{2 \mathrm{Na}}$;

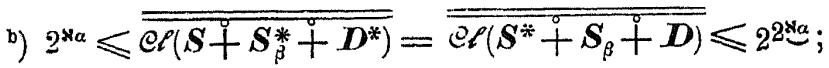

c) $2^{* a} \leqslant \overline{\overline{\mathcal{Q}\left(S_{\beta}+S_{\gamma}^{*}+D\right)}}=\overline{\overline{\mathscr{C} \mathcal{l}\left(S_{\gamma}+S_{\beta}^{*}+D^{*}\right)}} \leqslant 2^{2^{2}{ }_{\alpha}}$;

Démonstration. En vertu des th. $51^{\mathrm{b}}, 73^{\mathrm{d}}$ et du lem. $19^{\mathrm{b}, \mathrm{o}}$, on a les inclusions: $S_{\beta} \stackrel{+}{D} \check{C}^{\circ} S_{\beta}+S_{\gamma}^{*}+D \check{C}^{\circ} S^{*}+S_{\beta}+D \subset^{\circ} \boldsymbol{T}+S_{\text {, }}$ qui donnent en raison du th. $21^{\mathrm{a}}: \operatorname{eP}(\boldsymbol{T}+\boldsymbol{S}) \subset \mathcal{C} \mathcal{P}\left(\boldsymbol{S}^{*}+\boldsymbol{S}_{\beta}+\boldsymbol{D}\right) \subset$ $\subset \mathcal{Q} \mathcal{C}\left(\boldsymbol{S}_{\beta}+\boldsymbol{S}_{,}^{*}+\boldsymbol{D}\right) \subset \mathcal{Q} \mathcal{C}\left(\boldsymbol{S}_{\beta} \stackrel{+}{+} \boldsymbol{D}\right)$. Tenant compte du th. fond. V, on en obtient:

(1) $2^{\boldsymbol{N}_{\alpha}} \leqslant \overline{\overline{\mathcal{Q} \ell\left(\boldsymbol{S}^{*}+\boldsymbol{S}_{\beta}+\boldsymbol{D}\right)}} \leqslant \overline{\overline{\mathcal{Q} \ell\left(\boldsymbol{S}_{\beta}+\boldsymbol{S}_{\gamma}^{*}+\boldsymbol{D}\right)}} \leqslant \overline{\overline{\mathscr{Q} \mathcal{C}\left(\boldsymbol{S}_{\beta}+\bar{D}\right)}}$

D'après les th. $79^{\mathrm{a}}$ (pour $\gamma=\boldsymbol{\beta}$ ) et $21^{\mathrm{b}}, \mathscr{e P}\left(\boldsymbol{S}_{\beta}+\boldsymbol{D}\right)=$

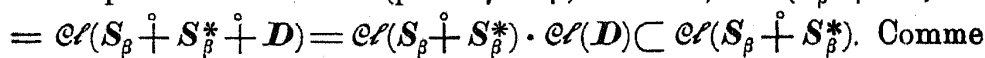
on a par hyputhèse $\beta>c f(\alpha)$, le th. 69 (pour $\gamma=\beta$ ) entraîne en outre: $\overline{\overline{\mathcal{C} P\left(\boldsymbol{S}_{\beta}+\boldsymbol{S}_{\beta}^{*}\right)}} \leqslant 2^{2^{\text {Na }}}$ On a, par conséquent,

$$
\overline{\overline{\mathcal{Q}}\left(\boldsymbol{S}_{\beta}+\bar{D}\right)} \leqslant 2^{\mathbb{R}^{*} \alpha} \text {. }
$$

En appliquant enfin à plusieurs reprises le th. 33 et le lem. $19^{\mathrm{b}, \mathrm{d}}$, on parvient aux égalités:

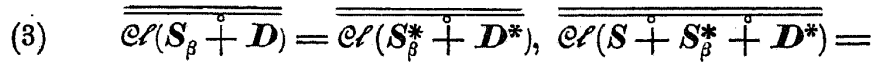

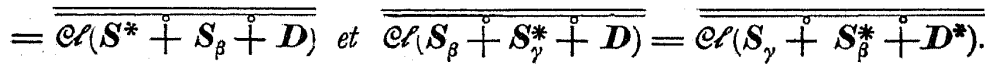

De (1)-(3) s'obtiennent immédiatement toutes les formules cherchées.

Théorème 83. Si $\overline{\bar{I}}=\kappa_{\alpha}$ et $\beta>c f(\alpha)$, on a $2^{\star \alpha} \leqslant \overline{\overline{\mathcal{Q} \ell\left(\boldsymbol{R}_{\beta}+\boldsymbol{D}\right)}}=$

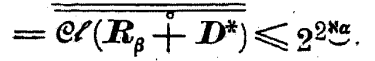

Démonstration est complètement analogue à celle du th. 71 .
Theorème fondamental XVI. L'hypothèse $H$ entraine les conséquences suivantes:

Si $\overline{\overline{1}}=\aleph_{\alpha}$ et $\beta>p(a)$, on $a$ :

a) $\overline{\overline{\mathscr{C}\left(S_{\beta}+D\right.}}=\overline{\overline{\mathscr{Q}\left(S_{\beta}^{*}+D^{*}\right)}}=2^{\mathrm{N \alpha}}$;

b) $\overline{\overline{\mathscr{C}}\left(\boldsymbol{S}+\boldsymbol{S}_{\beta}^{*}+\overline{D^{*}}\right)}=\overline{\overline{\mathscr{Q} \ell}\left(\boldsymbol{S}^{*}+\overline{\left.S_{\beta}+\bar{D}\right)}\right.}=2^{\mathrm{N \alpha}}$;

c) $\overline{\mathcal{Q} \ell\left(\boldsymbol{S}_{\beta}+\boldsymbol{S}_{\gamma}^{*}+\bar{D}\right)}=\overline{\overline{\mathcal{Q}}\left(\boldsymbol{S}_{\gamma}+\boldsymbol{S}_{\beta}^{*}+\overline{\left.D^{*}\right)}\right.}=2^{\mathrm{*} \alpha}$.

Théorème 84. L'hypothèse $H$ entraîne la conséquence suivante:

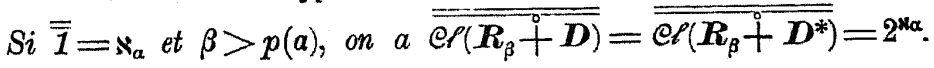

Démonstration des deux derniers théorèmes, basée sur les th. 82,83 et sur le lem. $9^{\mathrm{ab}}$, n'offre pas de difficultés (cf. le th. XII).

La comparaison des th. XIII ${ }^{b}$ el XIV montre que les familles $\mathcal{C}(\boldsymbol{S}+\boldsymbol{D})$ ei $\mathcal{Q} \mathcal{C}\left(\boldsymbol{S}^{*}+\boldsymbol{D}\right)$, que l'on est tenté de croire identiques, se comportent en réalité, même au point de vue de leur paissance, de façons essentiellement différentes. Pour les familles $\mathcal{Q}\left(\boldsymbol{S}_{\beta}+\boldsymbol{D}\right)$ et $\mathfrak{C} \mathcal{R}\left(\boldsymbol{S}_{\beta}^{*} \stackrel{+}{+}\right)$ le même effet est produit par les th. XIIr' et XVIa .

\section{Résumé.}

Le domaine de ces recherches comprend certaines opérations élémentaires qui font correspondre à toute classe d'ensemble des nouvelles classes d'ensembles, à savoir les operations $\boldsymbol{C}, \boldsymbol{T}, \boldsymbol{S}, \boldsymbol{S}_{\xi}$ (où $\xi$ parcourt tuus les nombres ordinaux) et $D$, ainsi que par leurs opérations doubles; la classe de toutes ces opérations à été désignée par $\Re($ th. 80 ). Les problèmes fondamentaux de cet ourrage consistent à déterminer la puissance des familles de toutes les classes qui se composent de sous-ensembles d'un ensemble donné 1 et qui sont closes par rapport à une quelconque des opérations indiquées ou bien par rapport à plusieurs de ces opérations à la fois; dans la symbolique introduite ci-dessus les familles en question se présentent sous la forme de $\mathcal{C}(\boldsymbol{F})$ où $\boldsymbol{F} \in \mathcal{S}_{\left(\tilde{F}^{\prime}\right) \text {. Dans les }}$ théorèmes fondamentaux des $\$ \S 3-7$ nous avons examiné une série de problèmes de ce genre et réussi (ayant parfois recours à l'hypothèse de $\mathrm{Cantor}$ sur les alephs) à déterminer les puissances des familles envisagées d'après la puissance $\aleph_{\alpha}$ de l'ensemble univer- 
sel 1. Les résultats acquis montrent que la puissance d'une famille examinée est égale le plus souvent soit à $2^{2 \mathrm{N \alpha}}$, soit à $2^{\mathrm{N \alpha}}$; cepen-. dant certaines des ces familles ne contenaient que 2 éléments.

Pour se rendre compte, en quelle mesure les problèmes de cegenre sont épuisés par nos recherches, on consultera le th. 80. Ce théorème montre que toutes les familles $\mathcal{C}\left(\boldsymbol{F}^{\prime}\right)$ où $\boldsymbol{F}_{\epsilon} \in(\mathfrak{S})$ se réduisent aux certains types, assez simples et peu nombreux. En rapprochant ce résultat des ceux qui ont été obtenus dans les th. fond. I-XVI, on constate que les recherchès présentes épuisent tous les, problèmes qui concernent la puissance des familles de toutes les classes closes par rapport à une opération arbitraire de la classe $\Re$ ou bien par rapport à plusieurs opérations de cette classe à la fois.

Il est intéressant que les résultats exposés ci-dessus se laissent étendre aux opérations d'une classe beancoup plus vaste que $\widetilde{S}(\Re)$, à savoir aux opérations de la classe $\mathfrak{B}(\Re)$ formée de toutes les opérations qui s'obtiennent de celles de la classe $\Re$ moyennant l'addition et la multiplication relative effectuées un nombre arbitraire (fini on transfini) des fois (cf. la déf. $11^{\%}$ ). Les opérations mêmes de la classe $\mathfrak{B}(\Omega)$ peuvent présenter une structure bien compliquée et elles ne se laissent pas rammener à un nombre fini de types simples. Il résulte par contre des remarques générales du $\$ 3$ (cf. th. B, p. 217) que les familles de la forme $\mathcal{C} \mathscr{P}(\boldsymbol{F})$ où $\boldsymbol{F} \in \mathfrak{B}(\mathfrak{R})$ se prètent à une réduction considérable et qu'elle aboulit aux-mêmes familles qui ont apparu au cours de l'examen de la classe $\mathfrak{S}(\mathfrak{I})$ dans, le th. 80 et à une seule nouvelle famille $\mathcal{E} \mathcal{L}(\boldsymbol{I})$, dopt la prissance nous est connue du th. 32 .

Certains résultats (th. $61,64,72,81,84$ ) concernaient en outre, au lieu de la classe $\mathfrak{R}$. une classe plus vaste, obtenne de $\Re$ par l'adjonction des opérations $\boldsymbol{R}_{\xi}$ (où $\xi$ parcourt tous les nombres ordinaux). Sans chercher à épniser dans l'exposé présent tous les problèmes de ce genre, je ne remarquerai ici que les problèmes dont je n'ai pas fait une mention explicite (p. ex. celui de la puissance de la famille $\mathcal{C}\left(\boldsymbol{S}_{\beta} \stackrel{+}{+} \boldsymbol{R}_{y}\right)$ ) se laissent résondre sans difficalté par les mélhodes appliquées dans cet ouvrage à plasieurs reprises.

\section{Indexe des symboles.}

J'énumère ici les symboles introduits pour la première fois ou non universellement admis, en indiquant le lieu où se trouve l'explication de lour sens:

$0,1, \Sigma(\boldsymbol{K}), \Pi(\boldsymbol{K}) \ldots \ldots . \ldots . . .81, \quad \ldots$ p. 183;

$J(a), \underset{f(x)}{E}[], \underset{f(x, y)}{E}[\mathrm{C}] \ldots \ldots \ldots$. . . $81, \quad$ p. 184; $\sup (A), \lim _{\xi<\alpha} \varphi_{\xi} \ldots \ldots \ldots . . .1_{1}$ déf. 1, p. 184; $c f(\alpha) . . . . . . . . . . .81$, . déf. 2, p. 184; $p(\alpha) . . . . . . . . . . . \$ 1$, déf. 3 , p. 187; $\mathfrak{a}^{\mathfrak{b}} \ldots \ldots \ldots \ldots \ldots$. . . . 1 , déf. 4 , p. 188; $U(A), \quad U_{a}(A), \quad V(A) \ldots \ldots \ldots . . . \$ 1$ déf. 5, p. 195 $\bar{f}(A) \ldots \ldots \ldots \ldots \ldots 1$, déf. 6 , p. 197 ; $\lim _{\xi<a} F_{\xi}, \overline{\lim }_{\xi<\alpha} F_{\xi}, \quad \lim _{\xi<a} F_{\xi} \ldots \ldots . . . \S 1$, déf. 7, p. 199; $\boldsymbol{F}=G, \quad \boldsymbol{F}^{\circ} \subset^{\circ}$. . . . . . . . . \& 2, déf. 8, p. 201; $\boldsymbol{F}+\boldsymbol{G}, \quad \sum_{x \in A}^{\circ} \boldsymbol{F}_{x} \ldots \ldots \ldots$. 2 , déf. 9, p. 201; $F G, \quad \prod_{\xi}^{0}, F^{\nu} \ldots \ldots . . . . \$ 2$, déf. 10, p. 202; $\mathfrak{S}(\mathfrak{F}), \mathfrak{B}(\mathfrak{F}), \mathfrak{B}(\mathfrak{F}) \ldots \ldots . . \ldots . . \$ 2$, déf. 11, p. 203; $\mathfrak{M}, \mathfrak{A}, \mathfrak{Z}_{\beta} \ldots \ldots \ldots \ldots$ I. . . . . . . . . . . . $\$$ 2, déf. 13, p. 209; C............... \$ 2 , déf. 14 , p. 210 $F^{*} \ldots \ldots \ldots \ldots$. . . . . $\$ 2$, déf. 15, p. 210 ; $\mathcal{O}\left(\boldsymbol{F}^{\prime}\right) \ldots \ldots \ldots$. . . . . . $\$ 3$, déf. 16, p. 212; T. . . . . . . . . . . $\$ 4$, dét. 17 , p. 227 S . . . . . . . . . . . . \$ 5 , déf. 18, p. 233 ; $\boldsymbol{M}$. . . . . . . . . . . . . . $\$ 55$, déf. 19, p. 236;

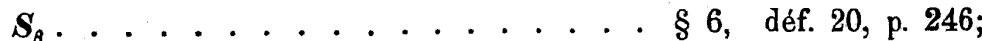
$\boldsymbol{R}_{\beta}$. . . . . . . . . . . \$ 6 , déf. 21, p. 254 D. . . . . . . . . . . . $\$ 7$, déf. 22, p. 287

Table des matières.

Intmotion

1. . . . . 183

§ 2. Algorythme des opérations sur les classes d'ensembles . 201 
§ 3. Notion générale de classe close par rapport à une opération donnée . . . . . . . . . . . . . . 211

§ 4. Opération $\boldsymbol{T}$. Classes d'ensembles héréditaires . . . . 227

$\S$ 5. Opération $S$. Classes d'ensembles additives . . . . . . 233

$\S 6$. Opération $\boldsymbol{S}_{\beta}$. Classes d'ensembles additives au dégré $\beta$. 246

\& 7. Opération $\boldsymbol{D}$. Classes d'ensembles soustractives . . . 287

Résumé . . . . . . . . . . . . . . . . . . . 301

Indexe des symboles. . . . . . . . . . . . . . . 303

Sur les éléments cycliques et leurs applications.

Par

C. Kuratowski et G. T. Whyburn ${ }^{1}$ ) (Lwów).

Dans l'ouvrage présent nous adoptons, comme point de départ une définition d'élément cyclique différente de celle adoptée primitivement ${ }^{2}$ ). Cela nous permet de développer la théorie d'éléments cycliques et de leurs applications sans avoir récours au théorème suivant, dont la démonstration est fort compliquée: si aucun point ne coupe l'espace Péanien ${ }^{3}$ ) entre deux points donnés $a$ et $b$, ces points sont situés sur une courbe simple fermée ${ }^{4}$ ).

Première Partie: Développement de la théorie.

\section{Définitions et propriétés générales d'espaces Péaniens.}

1 désigne l'espace considéré; nous le supposons toujours Péanien.

Le point $p$ (ou bien l'ensemble fermé $K$ ) coupe l'espace $E$ entre deux points $x$ et $y$, si $x$ et $y$ appartiennent à deux composantes différentes de $E-p$ (resp. $E-K$ ); une composante d'un ensemble $Z$ est un sous-ensemble connexe de $Z$ qui n'est contenu dans aucun autre sous-ensemble connexe de $Z$.

1) Fellow of the Jobn Simon Guggenheim Memorial Foundation.

2) d'après laquelle un élément cyclique est: soit un point qui coupe l'espace, soit un point d'arrêt (= point d'ordre 1), soit un continu saturé relativement à la propriété que tout couple de ses points s'y laisse unir par une courbe simple formée. Voir Whyburn, bibliographie N1 (à la fin de l'ourrage present).

3) = image continue de l'intervalle = espace compact, connexe et locaisment connexe. Il est à remarquer que la théorio d'éléments cycliques se laisse, en quelque sorte, étendre aux espaces non-compacts. Voir bibliogr. N22.

4) Théorème démontré par M. A y res, bibliogr. N17. Pour le cas du plan, N1.

Fundamenta Mathematicae. T. XVI. 